\title{
Krankenhäuser-spezifische Kosten- und Leistungsrechnung
}

- aktuelle Anwendung in Deutschland und Aufbau- sowie Übertragungsmöglichkeiten in andere Länder

(am Beispiel Syrien)

\section{Dissertation}

Zur Erlangung des wirtschaftwissenschaftlichen Doktorgrades der

Wirtschaftwissenschaftlichen Fakultät der Universität Göttingen

vorgelegt von

Basil Asaad

aus Syrien 


\title{
Krankenhäuser-spezifische Kosten- und Leistungsrechnung
}

-aktuelle Anwendung in Deutschland und Aufbau- sowie Übertragungsmöglichkeiten in andere Länder

(am Beispiel Syrien) -

\author{
Dissertation \\ Zur Erlangung des wirtschaftwissenschaftlichen Doktorgrades \\ der Wirtschaftwissenschaftlichen Fakultät \\ der Universität Göttingen
}

vorgelegt von

Basil Asaad

aus Syrien

Göttingen 2000 
Erstgutachter:

Zweitgutachter:

Tag der mündlichen Prüfung:
Prof. Dr. Dr. h.c. Jürgen Bloech

Prof. Dr. Helmut Brede

29. 05.2000 
Abbildungsverzeichnis

4

II

Tabellenverzeichnis. 10

III

Abkürzungsverzeichnis. 12

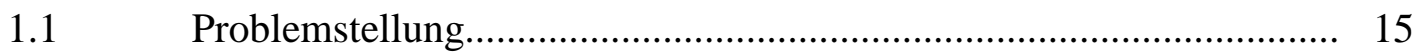

1.2 Zielsetzung der Arbeit........................................................... 16

Aufbau der Arbeit .................................................................. 17

2 DAS KRANKENHAUS ALS WIRTSCHAFTLICHER

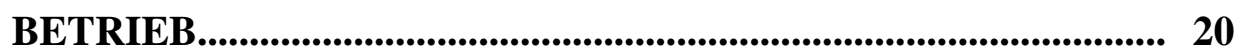

2.1 Allgemeine betriebswirtschaftlichen Betrachtungen an Krankenhaus................................................................................. 20

2.1.1 Begriffsdefinition und Arten von Krankenhäusern........................... 20

2.1.2 Das Zielsystem des Krankenhauses................................................ 24

2.1.3 Die Leistungen eines Krankenhauses............................................. 26

2.1.4 Aspekte der Wirtschaftlichkeit im Krankenhaus.............................. 32

2.1.5 Der Betriebsprozess des Krankenhauses........................................ 34

2.2 Krankenhäuser in Deutschland....................................................... 37

2.2.1 Das Krankenhauswesen in Deutschland.......................................... 37

2.2.1.1 Definition des deutschen Krankenhauses 38

2.2.1.2 Die gesetzlichen Rahmenbedingungen für deutsche Krankenhäuser

2.2.2 Wirtschaftlichkeit und Leistungsfähigkeit in deutschen Kranken-

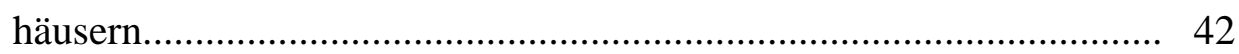

2.2.3 Finanzierungs- und Entgeltsystem der deutschen Krankenhäuser........ 44

2.2.3.1 Das Finanzierungssystem............................................................. 44

2.2.3.2 Das Entgeltsystem in deutschen Krankenhäusern............................. 45

2. 3 Das syrische Krankenhaus als wirtschaftlichen Betrieb................... 50

2. 3.1 Allgemeine Betrachtungen der syrischen Krankenhäuser ................. 50

2.3.2 Die Begriffsdefinition des syrischen Krankenhauses........................ 52 
2.3.3 Wirtschaftlichkeit und Leistungsfähigkeit in syrischen Krankenhäusern.............................................................................. 53

2.3.4 Finanzierungssystem in den syrischen Krankenhäusern.................... 54

3 GRUNDLAGE DER KOSTEN- UND LEISTUNGSRECHNUNG IM KRANKENHAUS .................................................. 55

3.1 Allgemeine Betrachtungen zur Kosten- und Leistungsrechnung in Krankenhaus........................................................................ 55

3.1.1 Aufgaben der Kosten- und Leistungsrechnung............................... 55

3.1.2 Begriffsdefinitionen zur krankenhausspezifischen Kosten- und Leistungsrechnung...................................................................... 60

3.1.2.1 Kostenbegriff.......................................................................... 61

3.1.2.2 Leistungsbegriff................................................................... 63

3.2 Kosten- und Leistungsrechnung in deutschen Krankenhäusern.......... 64

3.2.1 Aufgaben der Kosten- und Leistungsrechnung in deutschen Kran-

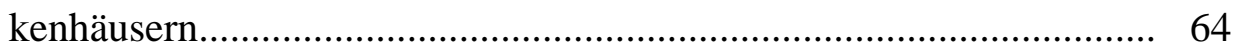

3.2.1.1 Die Kosten- und Leistungsrechnung als gesetzliche Dokumentations-

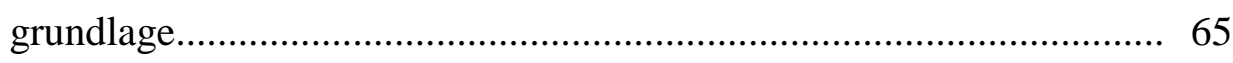

3.2.1.2 Die Kosten- und Leistungsrechnung als Instrument der Entscheidungsunterstützung.................................................................. 70

3.2.2 Rechtliche Rahmenbedingungen der Kosten- und Leistungsrechnung in deutschen Krankenhäusern........................................... 72

3.2.2.1 Allgemeine Vorschriften........................................................... 72

3.2.2.2 Spezielle Vorschriften.............................................................. 73

3.2.3 Begriffsdefinitionen zur Kosten- und Leistungsrechnung in

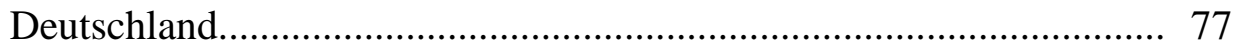

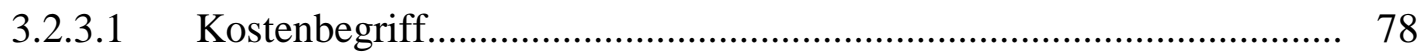

3.2.3.2 Leistungsbegriff................................................................. 80

3.2.4 Mindestanforderungen an die Kosten- und Leistungsrechnung im deutschen Krankenhaus.......................................................... 82 
3.3 Grundlagen der Kosten- und Leistungsrechnung in syrischen

Krankenhäusern

3.3.1 Rechtliche Rahmenbedingungen der Kosten- und Leistungsrechnung in syrischen Krankenhäusern.

3.3.2 Aufgaben der Kosten- und Leistungsrechnung in syrischen Krankenhäusern

3.3.3 Begriffsdefinition zur Kosten- und Leistungsrechnung in syrischen Krankenhäusern. 86

3.3.3.1 Der Kostenbegriff...................................................................... 86

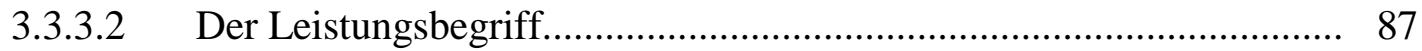

4 TEILGEBIETE DER KOSTEN- UND LEISTUNGSRECHNUNG........................................................................................ 88

4.1 Aufbau der Kosten- und Leistungsrechnung im Krankenhaus............ 88

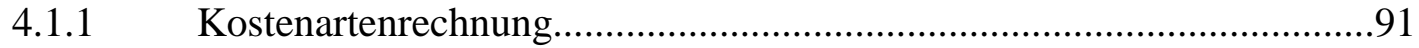

4.1.1.1 Definition und Aufgaben der Kostenartenrechnung........................... 91

4.1.1.2 Gliederung der Kostenartenrechnung............................................. 92

4.1.1.3 Erfassung der Kostenarten.......................................................... 96

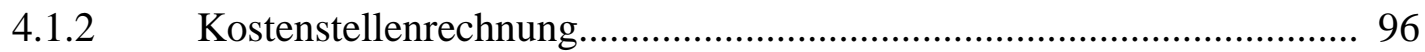

4.1.2.1 Definition und Aufgaben der Kostenstellenrechnung......................... 98

4.1.2.2 Bildung und Einteilung von Kostenstellen........................................ 100

4.1.2.3 Durchführung der Kostenstellenrechnung....................................... 106

4. $1.3 \quad$ Kostenträgerrechnung................................................................. 111

4.1.3.1 Definition und Aufgabe der Kostenträgerrechnung........................... 111

4.1.3.2 Identifikation von Kostenträgern. ................................................... 114

4.1.3.3 Durchführung der Kostenträgerstückrechnung................................. 115

4.1.3.4 Durchführung der Kostenträgerzeitrechnung.................................. 118

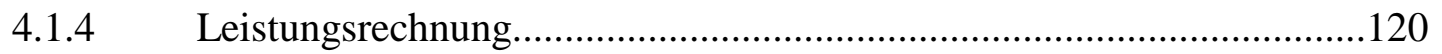

4.1.4.1 Definition und Aufgaben der Leistungsrechnung............................. 120

4.1.4.2 Durchführung der Leistungsrechnung............................................ 121

4.1.4.3 Erfassung der Leistungsarten und Leistungsstatistik......................... 121 
4.2 Teilgebiete der Kosten- und Leistungsrechnung in deutschen Krankenhäusern .................................................................... 123

4.2.1 Kostenartenrechnung in den deutschen Krankenhäusern................... 124

4.2.1.1 Definition und Gliederung der Kostenartenrechnung in den deutschen Krankenhäusern.................................................................... 124

4.2.1.2 Erfassung der Kostenartenrechnung im deutschen Krankenhaus......... 126

4.2.2 Kostenstellenrechnung in deutschen Krankenhäusern........................ 133

4.2.2.1 Definition und Aufgaben der Kostenstellenrechnung in deutschen Krankenhäusern........................................................................ 133

4.2.2.2 Bildung und Kontierung der Kostenstellen in deutschen Krankenhäusern

4.2.2.3 Durchführung der Kostenstellenrechnung in deutschen Krankenhäusern.

4.2.3 Kostenträgerrechnung in deutschen Krankenhäusern.

4.2.3.1 Definition und Aufgabe der Kostenträgerrechnung in deutschen Krankenhäusern

4.2.3.2 Bildung von Kostenträgern in den deutschen Krankenhäusern..... 145

4.2.3.3 Durchführung der Kostenträgerstückrechnung in deutschen Krankenhäusern.

4.2.3.3.1 Kalkulation von Fallpauschalen 149

4.2.3.3.2 Kalkulation von Sonderentgelten...................................................... 158

4.2.3.3.3 Kalkulation von Abteilungspflegesatz und Basispflegesatz............... 160

4.2.3.3.4. Durchführung der Kostenträgerzeitrechnung....................................... 161

4.2.4 Leistungsrechnung in den deutschen Krankenhäusern...................... 164

4.2.4.1 Definition und Aufgaben der Leistungsrechnung in deutschen Krankenhäusern.

4.2.4.2 Erfassung und Bewertung der Leistungen in deutschen Krankenhä

4.2.4.3 Leistungsstatistik in den deutschen Krankenhäusern........................ 167 
4.3 Teilgebiete der Kosten- und Leistungsrechnung in syrischen Krankenhäusern.

4.3.1 Kostenartenrechnung in syrischen Krankenhäusern......................... 171

4.3.1.1 Definition und Aufgaben der Kostenarten........................................ 171

4.3.1.2 Erfassung der Kostenartenrechnung in syrischen Krankenhäusern..... 172

4.3.2 Kostenstellenrechnung in syrischen Krankenhäusern....................... 175

4.3.2.1 Definition und Aufgaben der Kostenstellenrechnung in syrischen Krankenhäusern................................................................. 175

4.3.2.2 Bildung der Kostenstellenrechnung in syrischen Krankenhäusern...... 176

4.3.2.3 Durchführung der Kostenstellenrechnung in syrischen Kranken-

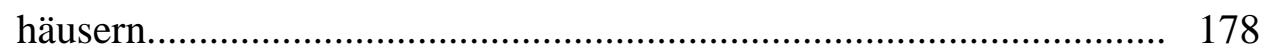

4.3.3 Kostenträgerrechnung in syrischen Krankenhäusern.......................... 179

4.3.3.1 Kostenträgerstückrechnung in den syrischen Krankenhäusern........... 179

4.3.3.2 Kostenträgerzeitrechnung in syrischen Krankenhäusern.....................179

5 SYSTEME DER KOSTEN- UND LEISTUNGSRECHNUNG IM KRANKENHAUS.................................................................. 180

5.1 Allgemeine Betrachtungen an Systeme der Kosten- und Leistungsrechnung im Krankenhaus........................................................... 181

5.1.1 Zeitbezogene Systeme.............................................................. 182

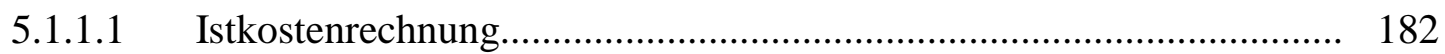

5.1.1.2 Normalkostenrechnung......................................................... 183

5.1.1.3 Plankostenrechnung............................................................... 184

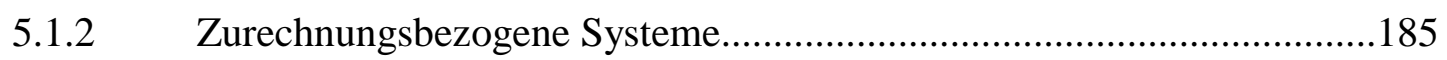

5.1.2.1 Vollkostenrechnung................................................................... 186

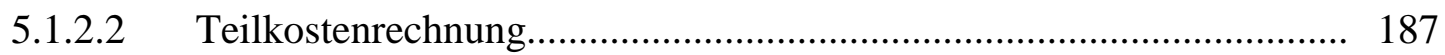

5.1.2.3 Ausgewählte Kostenverfahren in Krankenhaus................................. 188

5.1.3 Prozesskostenrechnung als modernes Kostenrechnungsverfahren....... 190

5.2 Systeme der Kosten- und Leistungsrechnung in deutschen Kranken-

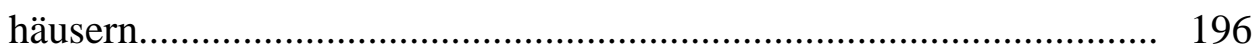

5.3 Systeme der Kosten- und Leistungsrechnung im syrischen Krankenhaus 
6.1 Allgemeine Betrachtungen...................................................... 200

6.2 die Notwendigkeit einer Kosten- und Leistungsrechnung sowie eines Kostenmanagements im Krankenhaus............................................... 204

6.3 Betrachtung des Krankenhausbetriebs in Deutschland und Beurteilung seiner Kosten- und Leistungsrechnung...................................... 208

6.4 Betrachtung und Empfehlungen für syrische Krankenhäuser............. 211

6.4.1 Modernes Management im syrischen Krankenhaus........................... 212

6.4.2 Aufbau- und Übertragungsmöglichkeiten einer Kosten- und .............. 212

Leistungsrechnung..................................................................... 215 
Abb. 1: Aufbau und Vorgehensweise der Arbeit................................................... 19

Abb. 2: Konstituive Merkmale des Krankenhauses.............................................. 22

Abb. 3: Zielsystem des Krankenhauses............................................................ 25

Abb. 4: Die Abgrenzung von Leistungskategorien................................................28

Abb. 5: Typologie der Dienstleistungsbetriebe.................................................. 29

Abb. 6: Schematische Darstellung des Leistungsgeschehens im Krankenhaus... 35

Abb. 7: Betriebsprozess des Krankenhauses..................................................... 37

Abb. 8: Finanzierungssystem des deutschen Krankenhauses............................... 45

Abb. 9: Vergütungssystem von Krankenhausleistungen der deutschen Kran-

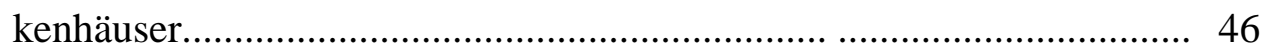

Abb. 10: Finanzierungssystem der privaten Krankenhäuser in Syrien.................. 54

Abb. 11: Finanzierungssystem der staatlichen Krankenhäuser in Syrien.............. 54

Abb. 12: Aufgabenschwerpunkte und Informationsempfänger des betrieblichen

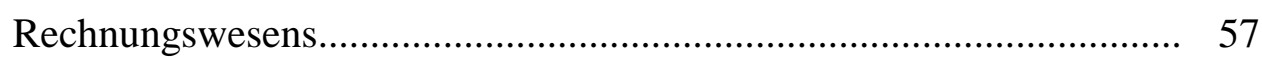

Abb. 13: Kosten und Leistung als einander bedingende Größen............................. 61

Abb. 14: Erstellung der Aufbauorganisation einer Krankenhaus- Kosten-

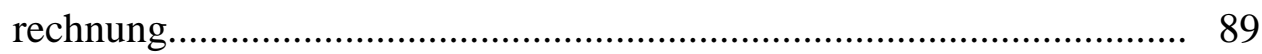

Abb. 15: Stufen und Phasen der Kostenrechnung............................................... 90

Abb. 16: Kostenartenplan für ein mittelgroßes Krankenhaus................................ 97

Abb. 17: Verantwortungsbereiche und Kostenbestimmungsfaktoren..................... 102

Abb. 18: Typen innerbetrieblicher Leistungsverflechtungen.................................. 108

Abb. 19: Verfahren der innerbetrieblichen Leistungsverrechnung......................... 109

Abb. 20: Der formale Aufbau des Betriebsabrechnungsbogens eines Kranken-

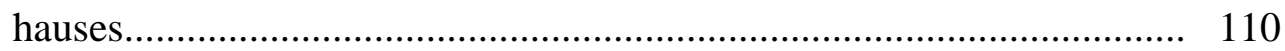

Abb. 21: Kostenträgerarten im Krankenhaus..................................................... 112

Abb. 22: Systematik der Kalkulationsverfahren................................................. 117

Abb. 23: die kurzfristige Erfolgsrechnung als erweiterte Kostenträgerzeitrechnung... 
Abb. 24: Kostenstellenrahmen für die Kosten- und Leistungsrechnung nach KHBV.

Abb. 25: Kostenstellenkontierungskatalog.

Abb. 26: Das Kalkulationsschema für die Ermittlung einer Fallpauschale.

Abb. 27: Das Kalkulationsschema der Kostenträgerzeitrechnung. 162

Abb. 28: Erlösarten im Krankenhaus

Abb. 29: Belegungsdaten des Krankenhauses. 167

Abb. 30: Versorgungsleistungen im Krankenhaus. 168

Abb. 31: Leistungsstatistik für medizinische Institutionen. 169

Abb. 32: Systeme der Kostenrechnung im Überblick. 181

Abb. 33: Teilprozesse im Krankenhaus. 193

Abb. 34: Brutto-Sozial-Produkt in Deutschland und Syrien. 202

Abb. 35: Krankenhäuser- und Bettenzahlin Deutschland und Syrien 203

Abb. 36: Pflegekosten in Deutschland und Syrien. 293

Abb. 37: Vergleich der Universitätsklinken Göttingen und Damaskus. 204

Abb. 38: Kostenartenstruktur des Krankenhauses 207 
Tab. 1: Aufgaben der Kosten- und Leistungsrechnung im Krankenhaus........... 59

Tab. 2: Abgrenzung von pagatorischen und kalkulatorischen Kosten.............. 79

Tab. 3: Bildungs- und Einteilungsmöglichkeiten der Kosten........................... 93

Tab. 4: Anteil des variablen und fixen Anteils bei ausgewählten Kostenarten... 95

Tab. 5: Verantwortungsbereiche des Krankenhauses...................................... 103

Tab. 6: Verantwortungsbereiche des Krankenhauses...................................... 105

Tab. 7: $\quad$ Kostenarten und Kostenstruktur..................................................... 126

Tab. 8: Dienstarten der Personalkosten........................................................ 128

Tab. 9: Löhne und Gehälter differenziert nach Dienstarten............................. 128

Tab. 10: Kostenarten und Struktur der Sachkosten............................................ 130

Tab. 11: Differenzierung und Struktur des medizinische Sachbedarfs................ 131

Tab. 12: Kalkulationsobjekte der Kosten- und Leistungs/Erlösrechnung und deren Vergütungsformen.............................................................. 147

Tab. 13: Kalkulationsergebnisse für die Abbildung 27: Zusammenstellung der Fallpauschale Nr. 17.02.

Tab. 14: Das Kalkulationsschema für die Ermittlung eines Sonderentgeltes (OP- Bereich).....

Tab. 15: Das Kalkulationsschema für Ermittlung eines Abteilungspflegesatzes.. 161

Tab. 16: Leistungserfassung des Krankenhauses............................................. 165

Tab. 17: Dienstarten der Personalkosten......................................................... 173

Tab. 18: Kostenarten im syrischen Krankenhaus............................................ 174

Tab. 19: Kostenstellen eines syrischen Krankenhauses.................................... 176

Tab. 20: Erlösarten im syrischen Krankenhaus................................................. 180

Tab. 21: Bezugsgrößen für Teilprozesses ,stationäre Krankenversorgung““........ 194 


\section{$\underline{\text { Abkürzungsverzeichnis }}$}

a.F.

Abb.

$\operatorname{AbgV}$

Abs.

ÄD

AHA

BAB

Bd.

BMÄ

BPflV

d. $h$.

DKG-NT

DM

DRG

EBM

EKG

F\&W

ff.

GAAP

GKV-NOG

GKV-SolG

GmbH

GOÄ

GoB

GRG

HGB

Hrsg.

i.e.S.

i.V.m.

ICD

ICPM

kalk

KG

KHBV

KHG

LKA alte Fassung

Abbildung

Abgrenzungsverordnung

Absatz

Ärtzlicher Dienst

American Hospital Association

Betriebsabrechnungsbogen

Band

Bundesmantelvertrag Ärzte

Bundespflegesatzverordnung

das heißt

Tarif der Deutschen Krankenhausgesellschaft für die Abrechnung erbrachter

Leistungen und für die Kostenerstatzung vom Arzt an das Krankenhaus

Deutsche Mark

Diagnosis Related Groups

Einheitliche Bewertungsmaßstab

Elektrokardiogramm

Führen und Wirtschaften im Krankenhaus

fortfolgend

Generally Accepted Accounting Principles

Krankenversicherung Neuordnungsgesetz

Krankenversicherungs-Solidaritätsgesetz

Gesellschaft mit beschränkter Haftung

Gebührenordnung der Ärzte

Grundsätze ordnungsmäßiger Buchführung

Gesundheitsreformgesetz

Handelsgesetzbuch

Herausgeber

Im engeren Sinne

in Verbindung mit

International Classification of Disease

International Classification of Procedures in Medicine

kalkulatorisch

Kommanditgesellschaft

Krankenhaus- Buchführungsverordnung

Krankenhausfinanzierungsgesetz

Leistungs- und Kalkulationsaufstellung 
It.

OP

PD

PMC

PPR

PT

S.

SERS

SGB V

Std.

SzU.

Tab.

u.a.

vgl.

WHO

z. B. laut

Operation

Pflegedienst

Patient Management Categories

Pflegepersonalregelung

Patient-Tag

Seite

Syrisches Einheitliches RechnungsSystem

Das Fünfte Sozialgesetzbuch

Stunde

Schriftenreihe zur Unternehmensführung

Tabelle

unter anderem

vergleiche

Weltgesundheitsorganisation

zum Beispiel 


\section{EINLEITUNG}

\section{1 Problemstellung}

Ein Blick auf die weltweite Entwicklung der Gesundheitssysteme zeigt, dass sie sich im letzten Jahrhundert durch große Fortschritte der Medizin qualitativ, quantitativ und strukturell tiefgreifend verändert haben. Bei dieser Entwicklung hat das Krankenhaus als Institution zunehmend eine zentrale Stellung erhalten. ${ }^{1}$

Diese Entwicklung hat auch den Gesundheitszustand der Menschen positiv verändert. Allerdings haben die steigenden Erwartungen an die Heil- und Therapiemethoden eine ständige Zunahme der Kosten des Gesundheitswesen zur Folge. Mit knappen finanzielle Ressourcen führt diese Situation zu einem Konflikt zwischen den medizinischen und wirtschaftlichen Zielen des Gesundheitswesens. Insbesondere bei den Krankenhäusern nehmen die Kosten auf Grund der ständig differenzierten Diagnosetechniken und Behandlungsverfahren zu. Damit wird weitergehend die Forderung gestellt, dass die Finanzierung und Steuerung des Krankenhauses nach den Mechanismen des Markts entschieden werden, ${ }^{2}$ wobei die Wirtschaftlichkeit als relevantes Prinzip gesehen werden muss.

Als Folge davon ist die Kostendämpfung im Krankenhaus ein Kernziel des Krankenhausmanagements geworden, wobei hier besonders beachten ist, dass die Maßnahmen der Kostenreduzierung die Qualität der Krankenhausleistungen nicht herabsetzen dürfen. Zur Erfüllung dieses Ziels ist der Aufbau und entsprechend die Entwicklung einer krankenhaus-spezifischen Kosten- und Leistungsrechnung eine notwendige Maßnahme. Im Einzelnen ist es jedoch ein Streitpunkt, welches Kosten- und Leistungsrechnungssystem in einem Krankenhaus geeignet ist. In der USA wurde beispielsweise diskutiert, ob ein Krankenhaus unter den allgemeinen Rechnungsprinzipien (Generally Accepted Accounting Principles GAAP) sein

\footnotetext{
${ }^{1}$ Vgl. Deutsche Krankenhausgesellschaft (1994 a): Positionen der DKG zur Reformdiskussion im Gesundheitswesen, die Stellung des Krankenhauses in der künftigen gesundheitlichen Versorgung, in: Das Krankenhaus, 12/1994, S. 539.

2 Deutz, W. (1999): Marketing als Erfolgsfaktor in Krankenhausmanagement, Frankfurt a. M., Berlin, New York, Paris, Wien: Lang, 1999, S. 2.
} 
Kostenrechnungssystem durchführen kann. ${ }^{3}$ Weiterhin ist zu klären, wie innerhalb des gewählten Systems welche Kosten auf die bezogenen Perioden bzw. Patienten aufgeteilt werden können.

In Deutschland hat der ständige Anstieg der Gesundheitskosten den Gesetzgeber zu häufigen Änderungen der Gesetze veranlasst, die mittlerweile das Krankenhaus als wirtschaftlicher Betrieb betrachten. Damit sind Krankenhäuser gezwungen worden, stärker als früher in Wettbewerb zu treten und Kostensenkungspotentiale zu realisieren. ${ }^{4}$

Im Gegensatz dazu erscheint das Problem in den Entwicklungsländen wie z. B. in Syrien im Vergleich mit den Industrieländern stärker, wo einerseits die Ressourcen wesentlich knapper sind und die Entwicklungsstufe der Wirtschaft und damit der Krankenhäuser niedriger ist bzw. eine Reihe von veralteten Gesetzen und staatlichen Verordnungen als Hindernisse für erfolgreiche Maßnahmen existieren. Anderseits hat sich die Betriebswirtschaftslehre erst im letzten Jahrzehnt in Richtung der Marktwirtschaft geöffnet. Insbesondere im Krankenhaus-Bereich gibt es fast keine Studien, die die Aspekte des Kosten- oder Betriebsmanagements der Krankenhäuser behandeln.

\subsection{Zielsetzung der Arbeit}

Ziel der Arbeit ist es, die Übertragbarkeit des deutschen Systems der Kosten- und Leistungsrechnung in Krankenhäusern auf syrische Verhältnisse herauszuarbeiten. Dazu wird das Krankenhaus insbesondere hinsichtlich der Kosten- und Leistungsrechnung betrachtet. Hierbei werden Krankenhäuser als wirtschaftliche Betriebe allgemein sowie die möglichen Anwendungen der Kosten- und Leistungsrechnung dargestellt. Nachfolgend wird die Situation in Deutschland und Syrien analysiert und beurteilt. Dies erscheint relevant, da hier zum ersten Mal in Deutschland ein Überblick über die Situation der syrischen Krankenhäuser darlegt wird. Weiterhin ist für Syrien die marktorientierte Betriebswirtschaftslehre relevant, und die in der

\footnotetext{
3 Finkler, S.(1994 a): Cost Accounting for Human Recourses in Hospitals, in: Finkler, S. A. (Edit.),Issues in Cost Accounting for Health Care Organisations, New York, 1994. S. 19.

${ }^{4}$ Vgl. Neubauer, G.: Kriterien zur Bewertung und Auswahl eines Krankenhaus-Vergütungssystems, in: das Krankenhaus, 10/ 1998, S. 578.
} 
deutschen Praxis aktuell angewendete Kosten- und Leistungsrechnung kann als Vorbild für Krankenhäuser betrachtet werden.

\section{3 Vorgehensweise und Aufbau der Arbeit}

Im zweiten Kapitel wird das Krankenhaus als wirtschaftlicher Betrieb betrachtet, wobei nach einer allgemeinen Begriffsdefinition das Krankenhauswesen in Deutschland inklusive der gesetzlichen Rahmenbedingungen und des Finanzierungs- und Entgeltsystems dargestellt wird. Nachfolgend wird die Situation der syrischen Krankenhäuser als wirtschaftliche Betriebe analysiert.

Das dritte Kapitel beschäftigt sich mit den Grundlagen der Kosten- und Leistungsrechnung im Krankenhaus. Hier wird ebenfalls zunächst eine Definition der Begriffe vorgenommen. Bei der Betrachtung der deutschen Krankenhäuser werden die Aufgaben der Kosten- und Leistungsrechnung als Dokumentationsgrundlage und als Instrument der Entscheidungsunterstützung sowie die rechtlichen Rahmenbedingungen dargestellt. Darüber Hinaus wird die Grundlegen sowie die Angegebenheiten der Kosten- und Leistungsrechnung in syrischen Betriebe erläutert. Grundsätzlich ist in syrischen Krankenhäusern die Durchführung einer Kosten- und Leistungsrechnung nicht vorgesehen.

In Kapitel 4 werden die Teilgebiete der Kosten- und Leistungsrechnung - Kostenarten-, -stellen- und -träger- und Leistungsrechnung - dargestellt. Im nachfolgenden Kapitel werden die Systeme der Kosten- und Leistungsrechnung erörtert, wobei zwischen zeitbezogenen (Ist- Normal und Plankostenrechnung) und zurechnungsbezogenen (voll- und Teilkostenrechnung) Systemen unterschieden wird. Dabei werden jeweils die aktuellen Anwendungen in deutschen Krankenhäusern sowie mögliche Anwendungen in Syrien untersucht.

Im abschließenden sechsten Kapitel wird die Notwendigkeit einer Kosten- und Leistungsrechnung sowie eines Kostenmanagements im Krankenhaus allgemein hergeleitet und der derzeitige Krankenhausbetrieb in Deutschland beurteilt. Nachfolgend werden Empfehlungen für eine Anwendung der Kosten- und Leistungsrechnung in syrischen Krankenhäusern gegeben, wobei die wirtschaftlichen Unterschiede zwischen Deutschland und Syrien herausgearbeitet werden. Aufgrund der 
Ähnlichkeiten des Krankenhaus-Prozesses erscheint jedoch eine Übertragung möglich.

In der gesamten Arbeit werden die Betrachtungen in drei Ebene vorgenommen: Zunächst werden die Aspekte theoretisch nach der Lage der betriebswirtschaftlichen Literatur des Krankenhauswesens, insbesondere bezüglich des Rechnungswesens, behandelt.

Zweitens werden aktuelle Anwendungen in Deutschland dargestellt, die gemäß den entsprechenden Gesetzen und Vorschriften praktiziert werden sollen.

Zuletzt wird die Situation der syrischen Krankenhäuser behandelt. Dabei ergibt sich aufgrund der fehlenden gesetzlichen und wissenschaftlichen Grundlagen die Schwierigkeit einer einheitlichen Betrachtung. Alternativ wurde die Praxis im Uniklinikum Damaskus untersucht und anhand der vorgefundenen Gegebenheiten die Durchführung einer Kosten- und Leistungsrechnung dargestellt. Dabei stellt das Syrische Einheitliche RechnungsSystem (SERS), das für alle wirtschaftlichen Betriebe gilt, eine Beschränkung dar.

Abb. 1 stellt der Aufbau der Arbeit in Zusammenhang mit dem Verfahren, das in den Kapiteln verfolgt wird, zusammenfassend dar. 


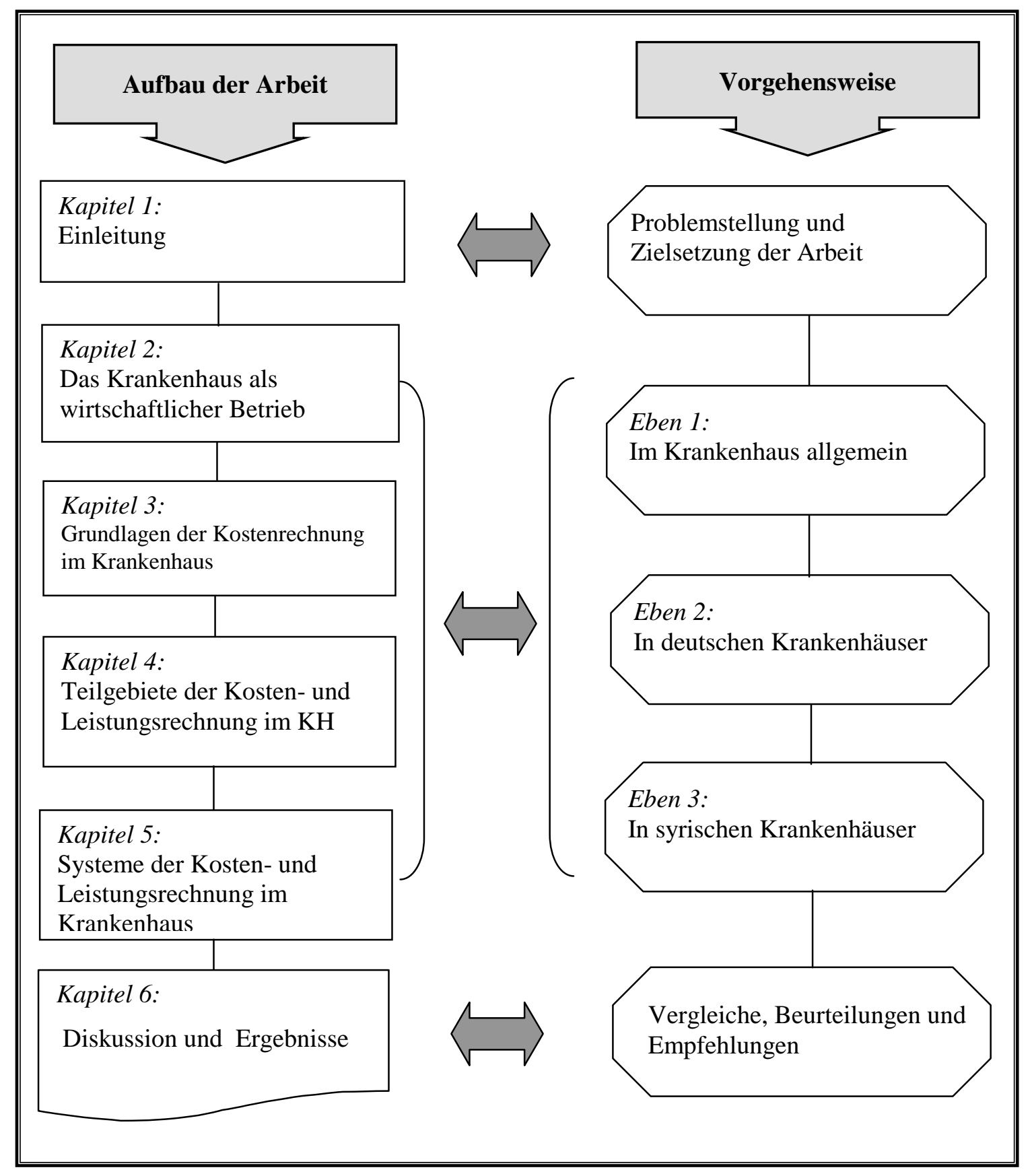

Abb. 1: Aufbau und Vorgehensweise der Arbeit. 


\section{DAS KRANKENHAUS ALS WIRTSCHAFTLICHER BETRIEB}

In diesem Kapital wird das Krankenhaus als wirtschaftlicher Betrieb in Betriebswirtschaftslehre, in Deutschland sowie in Syrien näher betrachtet, wobei insbesondere auf Aspekt der Kostenrechnung eingegangen wird.

\subsection{Allgemeine betriebswirtschaftlichen Betrachtungen an Krankenhaus}

Das Krankenhaus ist ein äußerst komplexes System. Die Kostenrechnung muss als Subsystem betrachtet werden, dessen Ausgestaltung am Gesamtsystem zu orientieren ist. Aus diesem Grund scheint es zwingend, zunächst den gesamten Krankenhausbetrieb mit seinen Zielen einführend zu betrachten, bevor für das Instrument „Kostenrechnung“ ein Sollkonzept entwickelt werden kann. Diese Betrachtung wird nur insoweit behandelt, als es für das Instrument „Kostenrechnung“ unmittelbare Bedeutung hat.

\subsubsection{Begriffsdefinition und Arten von Krankenhäusern}

Ausgehend von der Aufgabenstellung im Gesamtsystem der medizinischen, pflegerischen und sozialen Versorgung werden Krankenhäuser von der American Hospital Association (AHA) als Organisation definiert, die über eine geordnete medizinische Mannschaft und ständige medizinische Ausstattungen verfügt, wo den Patienten die benötigten Diagnosen, Therapie, Pflege und Versorgung gegeben werden. ${ }^{5}$

Die Weltgesundheitsorganisation (WHO) hat weitgehend das Krankenhaus definiert, das sowohl eine medizinische als auch eine soziale Institution darstellt, dessen Angebote im Haus erreicht werden können. Darüber hinaus stellt das Krankenhaus ein medizinisches und soziales Ausbildungs- und Untersuchungszentrum dar. Die WHO hat aber bemerkt, dass diese Definition nicht mit den praktischen Situationen der Krankenhäuser in vielen Entwicklungsländern übereinstimmt. Deswegen hat sie das Krankenhaus in diesen Ländern als Organisation

\footnotetext{
5 American Hospital Association (1974): Classification of Health Care Institutions, Chicago, 1974. S. 10 .
} 
definiert, in der für stationär aufgenommene Patienten medizinische und pflegerische Fürsorge gegeben wird. ${ }^{6}$

Allgemein sind Krankenhäuser sozial-technische, zielgerichtete, dynamisch offene, adaptive Systeme. Sie sind also Organisationen, die Entscheidungen über Ziele und Mitteleinsatz der Krankenversorgung treffen. Dabei sind Krankenhäuser eine besondere Erscheinungsform von Betrieben. ${ }^{7}$

Das Krankenhaus stellt eine Institution dar, deren hauptsächliche Aufgabe es ist, im Falle einer Krankheit den Zustand des betroffenen Menschen positiv zu beeinflussen. Die positive Beeinflussung des Gesundheitszustandes bedeutet die Verbesserung oder zumindest verhindert eine Verschlechterung des Gesundheitszustandes. Im Rahmen der Leistungserstellung werden Gesundheitsgüter hervorgebracht, die zu einer Verbesserung des Gesundheitszustandes beitragen sollen. Allerdings hat Krankenhaus sowohl ärztliche und pflegerischer Leistung als auch die Unterbringung und Verpflegung der Patienten zu sorgen. ${ }^{8}$

Nach dem Gesundheitskonzept der WHO wurde die Gesundheit so definiert, dass unter Gesundheit sowohl körperliches als auch soziales und seelisches Wohlbefinden verstanden wird. Dieses Konzept hat sich insofern durchgesetzt, als heute in der Forschung grundsätzlich zwischen physischer, psychischer und sozialer Gesundheit unterschieden wird. ${ }^{9}$

Dementsprechend kann eine Messung der Gesundheit beziehungsweise der Veränderung des Gesundheitszustandes auf diesen obengenannten drei Ebenen vorgenommen werden. Da es sich bei der Gesundheit also um eine mehrdimensionale Größe handelt, lässt sich die Verbesserung des Gesundheitszustands der Patienten nicht exakt bestimmen oder in eindeutigen messbaren Größen ausdrücken. Zudem

\footnotetext{
${ }^{6}$ Vgl. World Health Organization (1980): The Management of Hospitals, Technical Report Series, Geneva: WHO, 1980. S. 6-7.

7 Vgl. Hübner, H. (1980): Kostenrechnung im Krankenhaus, Grundlagen, Wirtschaftlichkeitsanalyse, Betriebsvergleich, 2. Aufl., Stuttgart, Berlin, Köln, Mainz, 1980, S. 22.

8 Vgl. Schwartz, A. (1997): Informations- und Anreizprobleme im Krankenhaussektor DUV., Wiesbaden, 1997. S. 11.

9 Vgl. Mooney, G. (1986): Economics, Medicine, and Health care, Sussex: Wheat sheaf Books, 1986, S. 22.
} 
ist die Einschätzung des Gesundheitszustandes stark von subjektiven Definitionen des Wohlbefindens durch das einzelne Individuum abhängig. ${ }^{10}$

Für eine differenzierte Betrachtung der Institution „Krankenhaus“ lässt sich eine Vielzahl von Unterscheidungskriterien heranziehen, das stellt Abb. 2 dar.

\begin{tabular}{|c|c|c|c|c|c|}
\hline Merkmale & \multicolumn{5}{|c|}{ Relevante Ausprägungen } \\
\hline Zielsetzung & \multicolumn{2}{|l|}{ For - Profit } & \multicolumn{3}{|c|}{ Non - Profit } \\
\hline Trägerschaft & $\begin{array}{l}\text { Privates } \\
\text { Krankenhaus }\end{array}$ & \multicolumn{2}{|c|}{$\begin{array}{l}\text { Freigemeinnütziges } \\
\text { Krankenhaus }\end{array}$} & \multicolumn{2}{|c|}{$\begin{array}{l}\text { Öffentliches } \\
\text { Krankenhaus }\end{array}$} \\
\hline $\begin{array}{l}\text { Versorgungsstuf } \\
\mathrm{e}\end{array}$ & $\begin{array}{l}\text { Grundversorg } \\
\text { ung }\end{array}$ & $\begin{array}{l}\text { Regelversorg } \\
\text { ung }\end{array}$ & $\begin{array}{l}\text { Schwerpur } \\
\text { ersorgung }\end{array}$ & ktv & $\begin{array}{l}\text { Zentralversorg } \\
\text { ung }\end{array}$ \\
\hline $\begin{array}{l}\text { Art der } \\
\text { Entlohnung } \\
\text { Festbesoldete }\end{array}$ & $\begin{array}{l}\text { Chefarzt- } \\
\text { Krankenhaus }\end{array}$ & \multicolumn{2}{|c|}{$\begin{array}{l}\text { Belegarzt- } \\
\text { Krankenhaus }\end{array}$} & \multicolumn{2}{|c|}{$\begin{array}{l}\text { Festbesoldeten- } \\
\text { Krankenhaus }\end{array}$} \\
\hline
\end{tabular}

Abb. 2: Konstituive Merkmale des Krankenhauses.

In der Literatur findet man häufig eine Einteilung in „For-Profit“" und „Non-Profit“Krankenhäuser, wobei die primäre Zielsetzung des Unternehmens als Kriterium herangezogen wird. For-Profit-Krankenhäuser als erwerbswirtschaftliche Betriebe sind primär auf die Erzielung von Gewinnen ausgerichtet und dadurch von NonProfit-Krankenhäuser als bedarfswirtschaftlicher Betrieb abzugrenzen, die an der Bedarfsdeckung orientiert sind. ${ }^{11}$ Das oberste Betriebsziel des Non-Profit- Krankenhauses kann man daher definieren mit Bedarfsdeckung der Bevölkerung des Einzugsbereiches mit adäquaten, qualitativ hochwertigen medizinischen Leistungen. ${ }^{12}$ Aber dieses Oberziel muss jedoch unter wirtschaftlichen Bedingungen erbracht werden. So ist im Krankenhauswesen ausdrücklich vorgesehen, dass Krankenhäuser ihre Verwaltung und Wirtschaftsführung planmäßig, wirtschaftlich und sparsam zu halten und somit Ausgaben zu vermeiden haben, die für den Betrieb, die

10 Vgl. Kaltenbach, T. (1993): Qualitätsmanagement in Krankenhaus, Qualitäts- und Effizienzsteigerung auf der Grundlage des Total Quality Management, 2. Aufl., Melsunger, 1993, S.665.

11 Vgl. Aldag, H. (1988): Rahmenbedingungen, Problemlösungserfordernisse und konzeptionelle Grundlegung eines Marketing für Krankenhäuser, Dissertation, Göttingen, 1988, S. 6.

12 Vgl. Schäfer, G. (1988): Betriebswirtschaftliche Aspekte in der Krankenhausfinanzierung, herausgegeben von der Fachvereinigung der Verwaltungsleiter deutscher Krankenanstalten e. v., Marl, 1988.S. 19. 
Erhaltung und die Erweiterung der Krankenanstalt nicht unbedingt erforderlich sind.

Non-Profit-Krankenhäuser unterliegen aber nicht den Regeln der Marktwirtschaft. Ausgaben, Kosten und Finanzierung richten sich nicht nach Angebot und Nachfrage, sondern werden zwischen dem Krankenhaus und seinen Sozialpartnern ausgehandelt.

Ein weiteres wichtiges Charakteristikum stellt die Trägerschaft dar. Hierbei lassen sich private und öffentliche Träger unterscheiden. Private Träger können wie im Fall der privaten Krankenhäuser - natürliche Personen oder juristische Personen des Privaten Rechts sein. ${ }^{13} \mathrm{Zu}$ den privaten Trägern zählen aber auch kirchliche Träger oder Träger der freien Wohlfahrtspflege wie Stiftungen oder Vereine. Krankenhäuser, die in diese Trägerkategorie fallen, werden als freigemeinnützige Krankenhäuser bezeichnet. Der Wirtschaftsgrundsatz, der diesen Krankenhäusern zugrunde liegt, entspricht dem karitativen oder humanitären Prinzip.

Öffentliche Krankenhäuser werden von öffentlichen Trägern wie die Staat bzw. Gebietskörperschaften (Länder, Kreise, Kommunen), Zweckverbänden von Gebietskörperschaften oder Sozialleistungsträgern (z.B. staatliche Versicherungsanstalt) nach dem Prinzip der Daseinsfürsorge betrieben und unterhalten. ${ }^{14}$

Eine Zuordnung der Krankenhäuser zu verschiedenen Versorgungsstufen kann nach Breite und Tiefe des medizinisch-pflegerischen Leistungsspektrums und der in Bettenzahlen ausgedrückten Größe des Krankenhauses erfolgt werden. Hierbei findet man Krankenhäuser für Grund-, Regel-, Schwerpunkte- und Zentralversorgung.

Ein weiteres Unterscheidungskriterium ist die Art der Entlohnung der leitenden Ärzte. Hiernach lassen sich Chefarzt-, Belegarztkrankenhäuser und Krankenhäuser mit festbesoldeten Ärzten unterscheiden.

\footnotetext{
13 Vgl. Fischer, D. (1988): Marktstruktur und Marktverhalten in der Krankenhauswirtschaft, Spardorf, 1988, S. 54.

${ }^{14}$ Vgl. Aldag, H. (1988), S. 6.
} 


\subsubsection{Das Zielsystem des Krankenhauses}

Alle Entscheidungen im Krankenhaus sollen einem möglichst geschlossenen, präzisen und widerspruchsfreien Wertsystem unterworfen sein, um rationales Handeln zu gewährleisten. Dieses Wertsystem wird „Zielsystem“ genannt. Ein Zielsystem dient der Beurteilung und Auswahl von Handlungsalternativen. ${ }^{15}$

Die Ziele im Krankenhaus selbst können wiederum nur unter Einordnung in das übergeordnete Gesundheitsversorgungssystem gesehen werden. Erst eine Operationalisierung der vorgegebenen und für sich selbst wenig aussagefähigen Zielen des Gesundheitssystems führt zu erreichbaren Zielen.

Im Gegensatz zum privaten Erwerbsbetrieb, deren Leistungsaufgaben - wie die Produktion von Waren oder Dienstleistungen - in ersten Linie der Gewinnzielung dienen, stellt sich die Situation in den meisten Krankenhäusern völlig anders dar. Einerseits dienen hier die Leistungsaufgaben in ihrer ursprünglichen Form allein der Erfüllung des Betriebszwecks. Die Mehrzahl der Krankenhäuser hat in ihrer Eigenschaft als Wirtschaftsbetriebe im Vergleich zu Industrieunternehmen eine strategische Dimension weniger. Anderseits ist das Krankenhaus dadurch geprägt, dass ihm seine Ziele meistens von anderen vorgegeben werden. Das wichtigste Ziel ist das Gewährleistungsziel. Staat oder öffentliche Gebietskörperschaften als Träger streben einen politisch bemessenen Zustand der Versorgung mit Krankenhausleistungen an. ${ }^{16}$

Das Hauptziel des Krankenhausbetriebs ist die Deckung des Krankenverbesserungsbedarfs der Bevölkerung an voll- und semistationären Krankenhausleistungen. Je nach den Gegebenheiten und Bedingungen des einzelnen Krankenhauses wird dieses Hauptziel durch Nebenziele ergänzt, die parallel zum Hauptziel angestrebt werden. Hier zu zählen u.a. Repräsentation des Krankenhausträgers, die Erzielung von Einkünften, die Sicherung der Arbeitszufriedenheit des Krankenhauspersonals, medizinische Forschung sowie Aus- und Weiterbildung des Gesundheitspersonals.

\footnotetext{
15 Vgl. Eichhorn, S. (1987): Krankenhausbetriebslehre: Theorie und Praxis der KrankenhauLeistungsrechnung, Kohlhammer(Hrsg.), Bd.3, Köln, Stuttgart, Berlin, Mainz, 1987 S. 14.

${ }^{16}$ Vgl. Richter, H. (1997), S. 17.
} 
Als Sachziel eines Krankenhausbetriebs kann mithin die Verbesserung der vollund teilstationären der Intensiv-, Normal-, Langzeit- und Teilzeitversorgung angesehen werden. Sie umfasst die Teilleistungen der ärztlichen Behandlungen, pflegerischen Betreuung, sozialen Fürsorge, seelsorgerischen Hilfe und Hotelversorgung. Hinzu können ambulante Krankenversorgung sowie Lehre und Forschung treten. Abb. 3 stellt das Zielsystem des Krankenhauses dar.

\section{Wirtschaftsgrundsätze des Krankenhausträger}

- Speziell-formal

Prinzip der Daseinsvorsorge, Caritas/ Humanitas

- Allgemein-formal Leistungsfähigkeit, Wirtschaftlichkeit, finanzielles

Gleichgewicht

\section{Zielkonzeption Krankenhausbetrieb}

- Sachziel

- Formalziel

Krankenversorgung

Bedarfsdeckung

institutioneller Sinn

\section{Zielsystem Krankenhausbetrieb}

- Hauptziel

Deckung des Bedarfs der Bevölkerung an voll- und teilstationärer Krankenhausversorgung

- Nebenziele

Sicherung der Arbeitszufriedenheit des Krankenhauspersonals, Aus- und Weiterbildung, Forschung, Erzielung von Einkünften.

- Zwischen- und Unterziele

- Leistungserstellungsziel

Betriebsführungsziel, Ziel der Leistungsfähigkeit, Ziel der Kostenwirtschaftlichkeit

- Bedarfsdeckungsziel Ziel der dringlichkeitsgemäßen Bedarfskongruenz, Ziel der räumlichen Bedarfskongruenz, Ziel der zeitlichen Bedarfskongruenz

- Personalwirtschaftsziel

- Finanzwirtschaftsziel Ziel der Sicherung des Personalbestandes, Ziel der Sicherung der Arbeitszufriedenheit und der Leistungen des Personals, Ziel der Sicherung der Personaleffizienz Liquiditätssicherungsziel, Ziel der Sicherung der Eigenwirtschaftlichkeit, Ziel der Sicherung der funktionellen Kapitalerhaltung

- Angebotswirtschaftsziel

- Autonomie- und Integrationsziel Ziel der optimalen Preisgestaltung, externes Kontaktziel, externes Informationsziel

Ziel der Entscheidungs- und Handlungsautonomie, Ziel der Kooperation mit anderen Krankenhäusern, Medizinbetrieben und sonstigen Betrieben

Abb. 3: Zielsystem des Krankenhauses. ${ }^{17}$

${ }^{17}$ In Anlehnung an Eichhorn, S. (1987) S. 15 


\subsubsection{Krankenhausleistungen}

Im Allgemein wird im Zusammenhang mit betriebswirtschaftlichen Überlegungen unter „Leistung“ das Ergebnis der betrieblichen Betätigung verstanden. Leistung ist „,Sachzielbezogene Entstehung von Gütern materieller oder immaterieller Art““. ${ }^{18}$ Der Leistungsbegriff wird daher von zwei Merkmalen bestimmt:

- Güterentstehung, d.h. eine Leistung liegt nur dann vor, wenn in einem Betrieb neue Wirtschaftsgüter entstehen,

- Zweckbestimmung, d.h. zur Leistung rechnen nur solche Güterentstehungen, die aus dem Prozess der betrieblichen Leistungserstellung hervorgegangen sind und die dem angestrebten Sachziel des Betriebes entsprechen.

Die Definition der Krankenhausleistung ergibt sich aus der vorangegangenen Formulierung der Aufgaben eines Krankenhauses. Entsprechend sind Krankenhausleistungen sämtliche Anstrengungen, die der Erfüllung eben jener Aufgaben dienen: Das Krankenhaus erbringt eine Leistung, wenn es den einer stationären Behandlung bedürftigen Patienten nach Maßgaben des medizinischen Kenntnisstandes und unter Berücksichtigung des medizinischen Fortschritts behandelt. ${ }^{19}$ Die Kernaufgaben, das Erkennen, Heilen, Bessern oder Lindern von Krankheiten, Leiden, Körperschaden der das Krankenhaus aufsuchenden Patienten wird durch Leistungen erfüllt, die das Krankenhaus unmittelbar für den Markt erbringt. ${ }^{20}$

Im ersten Schritt sind die Krankenhausleistungen in drei Kategorien zu unterteilen, und zwar die Bereithaltung von Krankenhausbetten (Vorhalteleistung), die medizinischen Behandlung von Patienten und die pflegerische Betreuung der Patienten einschließlich der reinen Hotelleistungen. ${ }^{21}$

18 Vgl. Hörmann, W., Ingruber, H. (1988): Krankenhausbetriebslehre: Grundzüge der Betriebsführung im Krankenhaus, 1. Aufl., Wien, 1988, S. 18.

${ }^{19}$ Vgl. Wiedermann, R. (1980): The Management of Hospitals, Technical Report Series, Geneva: WHO, 1980, S. 5.

${ }^{20}$ Vgl. Kaltenbach, T. (1993), S. 45.

${ }^{21}$ Die Bereithaltung von Krankenhausbetten wird am besten durch die Zahl der Betten ausgedrückt. Die medizinischen Behandlung wird durch die Zahl der Behandlungsfälle repräsentiert. Und die pflegerische Betreuung der Patienten siegelt sich in der Zahl der Pflegetage wieder. Vgl. Herbold, W., Horstmann, P., Gemke, H., Diek, M. (1997): Leistungsstrukturveränderungen - Nullrunde oder Budgeterhöhung?, in: Das Krankenhaus, 7/ 1997, S. 533. 
Das gesamte Spektrum der Leistungen des Krankenhauses läßt sich wiederum in Untergruppen der unterschiedlichen Ressourcenaufwendigkeit wie Diagnose-, Therapie-, Pflege- und Versorgungsleistungen aufgeteilt werden. ${ }^{22}$ Diagnoseleistungen betreffen ärztliche Einzelleistungen zur Feststellung und Benennung von Krankheiten. Therapieleistungen umfassen solche Einzelleistungen, die der Heilbehandlung des aufgenommenen Patienten dienen. Pflegeleistungen erfolgen im Bereich der Grundpflege (allgemeine Pflege) und der Behandlungspflege (spezielle Pflege). ${ }^{23}$ Einzelleistungen der Grundpflege dienen unabhängig vom Krankheitsbild der Befriedigung normaler Lebensbedürfnisse des Patienten und seiner psychischen und sozialen Betreuung. Die Behandlungspflege umfasst Pflegeleistungen, die im Zusammenhang mit der Diagnose und Therapie erbracht werden. ${ }^{24}$ Versorgungsleistungen betreffen Aufgaben der Versorgung und Verwaltung, die sich aus dem stationären Aufenthalt des Patienten ergeben. ${ }^{25}$ Die beispielhafte Aufgliederung des primären Leistungsspektrums zeigt Abb. 4:

\footnotetext{
${ }^{22}$ Vgl. Hörmann, W., Ingruber, H. (1988), S. 249.

${ }^{23}$ Vgl. Pschyrembel, W. (1986): Klinisches Wörterbuch, Berlin, 1986, S. 347 und S. 654.

24 Vgl. Eichhorn, S. (1975): Krankenhausbetriebslehre - Theorie und Praxis des Krankenhausbetriebes, Band. I, 3. Aufl., Stuttgart, Köln, Berlin, Mainz, 1975, S. 364.

${ }^{25}$ Vgl. Schmitz, R. (1993): Patientenbezogene Steuerung in Krankenhaus, Stuttgart, 1993, S. 38.
} 


\begin{tabular}{|c|c|c|}
\hline I. & $\begin{array}{r}\text { Diagnoseleistungen } \\
\qquad- \\
- \\
-\end{array}$ & $\begin{array}{l}\text { - Laboratoriumsdiagnostik } \\
\text { Röntgendiagnostik } \\
\text { Endoskopie } \\
\text { Sonographie }\end{array}$ \\
\hline II. & Therapieleistungen & $\begin{array}{l}\text { - Operationen } \\
\text { Strahlentherapie } \\
\text { Physikalische Therapie } \\
\text { Medikamentöse Therapie }\end{array}$ \\
\hline III. & Pflegeleistungen & $\begin{array}{l}\text { - Grundpflege } \\
\text { - Körperpflege und Ernährung } \\
\text { - Hilfestellung bei Ausscheidungen } \\
\text { - Bewegung und Lagerung } \\
\text { Behandlungspflege } \\
\text { - Assistenz bei diagnostischen } \\
\quad \text { Leistungen } \\
\text { - Assistenz bei therapeutischen } \\
\text { Leistungen } \\
\text { - Vitalzeichenkontrolle } \\
\text { Arzneimittelgabe } \\
\text { Wundbehandlung }\end{array}$ \\
\hline IV. & Versorgungsleistungen & $\begin{array}{l}\text { - Unterbringungsleistung } \\
\text { Speisen und Wäscheversorgung }\end{array}$ \\
\hline
\end{tabular}

Abb. 4: Die Abgrenzung von Krankenhaus-Leistungskategorien ${ }^{26}$

Daneben können sonstige Leistungen wie z. B. Schule im Krankenhaus, Krankenhausbibliothek, Krankenhaus-Cafe oder Shop im Klinik als Zusatznutzen bezeichnet werden. ${ }^{27}$

Nach der Art der betrieblichen Leistungen gehört das Krankenhaus mithin zur Gruppe der Dienstleistungsbetriebe. Innerhalb dieser Gruppen handelt es sich bei der Krankenhausleistung um einen der so genannten kundenpräsenzbedingten Dienstleistungsbetrieben „Gastbetrieben“. ${ }^{28}$ Abb. 5 stellt die Typologie der Dienstleistungsbetriebe dar.

26 Quell: Preuß, O. (1996): Kosten und Deckungsbeitragsmanagement im Krankenhaus unter besonderer Berücksichtigung von Fallpauschalen und Sonderentgelten, Lang, P. (Hrsg.), Frankfurt am Main, Berlin, Bern, New York, Paris, Wien, 1996, S. 37.

${ }^{27}$ Vgl. Deutz, W. (1999): Marketing als Erfolgsfaktor in Krankenhausmanagement, Frankfurt a. M., Berlin, Neu York, Paris, Wien: Lang, 1999, S. 48-49.

${ }^{28}$ Vgl. Hörmann, W., Ingruber, H. (1988) S. 7. 


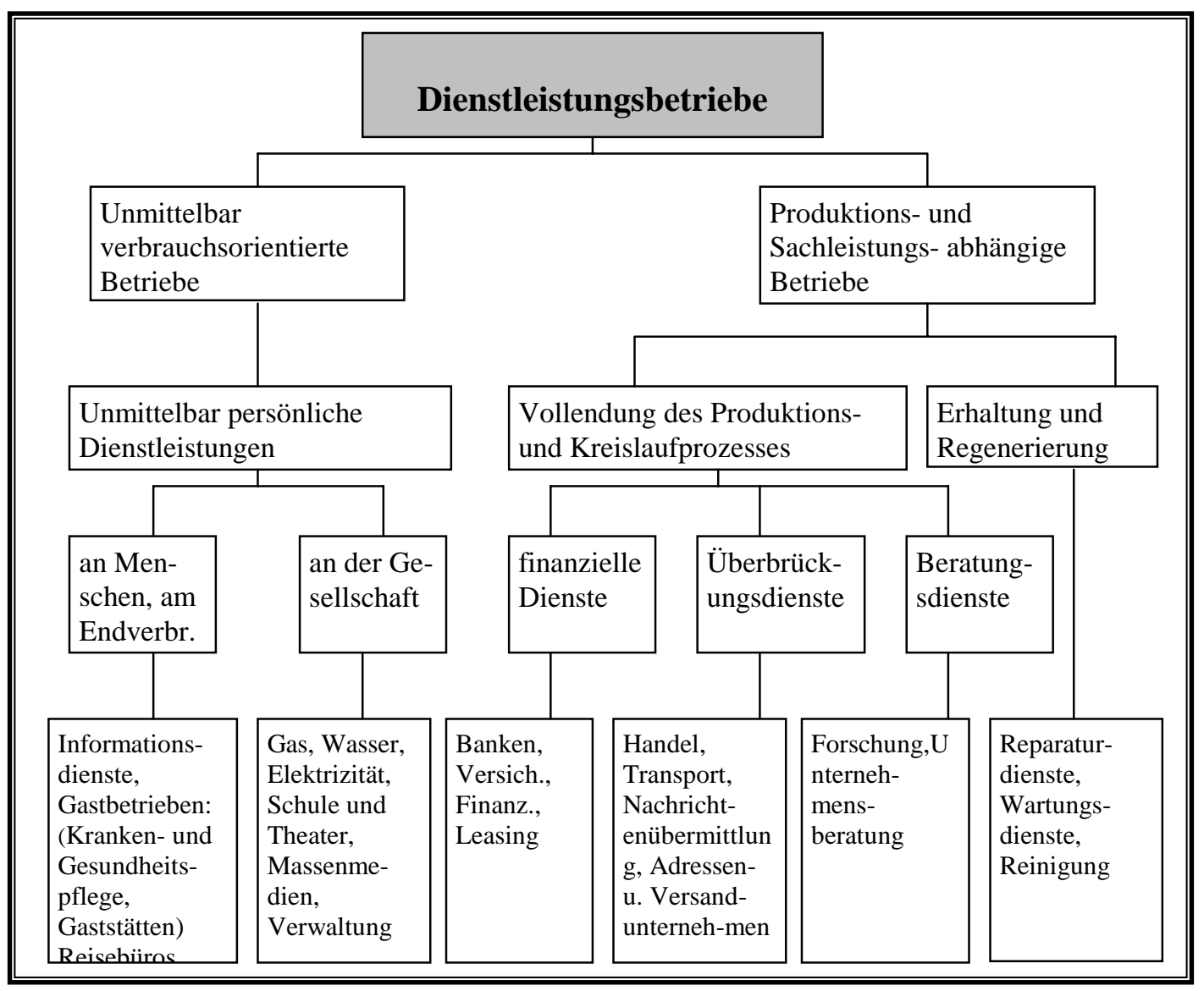

Abb. 5: Typologie der Dienstleistungsbetriebe ${ }^{29}$

Man spricht in diesem Zusammenhang auch von beiderseitig personenbezogenen Dienstleistungen, da die Erstellung und die Inanspruchnahme der betrieblichen Leistungen die Präsenz des Kunden erfordert. ${ }^{30}$ Krankenhausleistungen sind somit personengebundene Dienstleistungen. Bei den personengebundenen Dienstleistungen ist das Auffinden geeigneter Leistungskategorien mit erheblichen Problemen verbunden. Die Mengenkomponente lässt sich bei Dienstleistungen nur bedingt definieren und abgrenzen. Sie können daher nur schwer operationalisiert oder gemessen werden. Erschwerend kommt hinzu, dass bei Leistungsbeginn der Leistungsumfang nicht eindeutig absehbar ist. ${ }^{31}$

${ }^{29}$ Quelle: Keun, F. (1997): Einführung in die Krankenhauskostenrechnung, 3. Überarbeitete Aufl., Wiesbaden: Gabler, 1997, S. 2.

${ }^{30}$ Vgl. Berekoven, L. (1985): der Leistungsmarkt- Sachliche und empirische Befunde, in: Pestel (Hrsg.), 1985, S. 28.

31 Vgl. Herder-Dorneich, Ph. (1981): Problemgeschichte der Gesundheitsökonomik, in: HerderDorneich, Thiemeyer (Hrsg.), 1981., S. 29 ff. 
Der Konsument nimmt im Krankenhaus die Dienstleistung zur Wiederherstellung oder Verbesserung seines eignen Gesundheitszustandes in Anspruch. Dabei werden Krankenhausleistungen in der Regel folgende Besonderheiten zugeschrieben:

- Erstellung und Konsum erfolgen simultan: Diese Simultaneität bezieht sich auf den Umstand, dass Dienstleistungen im Leistungserstellungsprozess „uno-acto“ konsumiert werden. ${ }^{32}$ Das Uno-acto-Prinzip beschreibt den Umstand, dass schon mit der Erbringung der Leistung die Vertragserfüllung bzw. die Konsumtion der Leistung beginnt. Das bedeutet allerdings nicht, dass der Konsument während des Leistungserstellungsprozesses immer anwesend sein muss, ${ }^{33}$ obwohl dies bei einer Reihe von Dienstleistungen (wie bei der Krankenhausleistung) zwangsläufig der Fall ist.

Krankenhausleistungen sind damit weder lager- noch transportfähig. Sie müssen zum Zeitpunkt und am Ort der Erstellung der Nachfrage produziert werden. Zudem müssen Nachfragespitzen absorbiert werden können. Dadurch steigt der Anteil der Bereithaltungskosten an den Gesamtkosten des Krankenhauses. ${ }^{34} \mathrm{Im}$ Prinzip kann ein Krankenhauses in mancher Hinsicht mit der Feuerwehr vergleichen werden, deren Kosten im Wesentlichen unabhängig davon entstehen, ob es brennt oder nicht. Alle Kosten, die im Krankenhaus zur Aufrechterhaltung seiner Betriebsbereitschaft anfallen, entstehen, unabhängig von der erwarteter Auslastung. ${ }^{35}$

- Dienstleistungen werden unter Einbringung eines externen Faktors erbracht: Diese Integration eines externen Faktors bezeichnet die Tatsache, dass die Leistungserstellung nur möglich ist, wenn der Konsument sich selbst oder eines seiner Güter an dem Prozess beteiligt, ${ }^{36}$ d.h. Leistungserbringung im Krankenhaus bedingt die Mitwirkung des Patienten. Bei der Leistungserstellung

\footnotetext{
32 Vgl. Stauss, B. (1989): Beschwerdepolitik als Instrument des Dienstleistungsmarketing, in: Jahrbuch der Absatz- und Verbrauchsforschung, 1989, S. 48.

${ }^{33}$ Vgl. Meyer, D. (1993): Technischer Fortschritt im Gesundheitswesen, Tübingen, 1993, S. 25.

34 Vgl. Riebel, P. (1982): Grundfragen der Kostenrechnung im Gesundheitswesen und ihre Folgerungen für die Ordnungspolitik, in: Gäfgen, Lampert (Hrsg.), 1982, S. 66.

35 Vgl. Hildbrand, R. (1988), S. 377.

${ }^{36}$ Vgl. Wiedermann, R. (1998), S. 24.
} 
arbeiten Produzent und Konsument unmittelbar zusammen. ${ }^{37}$ Daraus ergeben sich verschiedene Konsequenzen für die Leistungserstellung und Leistungsmessung im Krankenhaus. Verweigert z.B. der Patient seine Mitwirkung, so kann der Gesundungsprozess im Krankenhaus erfolglos bleiben. ${ }^{38}$ Die Endkombination der Produktionsfaktoren wird somit auch durch die Beteiligung des Patienten bestimmt. Diese führt zum Problem, dass die Krankenhausleistung nicht ohne Weiteres von der Patientenleistung getrennt werden kann. ${ }^{39}$ Falls man die Leistung alleine dem Krankenhaus zurechnet, ergeben sich Ungleichheiten durch die unterschiedliche Mitwirkung des Patienten.

- Beschränkte Substitutionalität der Produktionsfaktoren: Der Betriebsprozess im Krankenhaus wird nicht nur von Menschen getragen, sondern er betrifft auch unmittelbar den Menschen (den Patienten). Die Kundenpräsenz und die Unmöglichkeit, immaterielle Bestandteile der Krankenhausleistung durch Maschinen $\mathrm{zu}$ ersetzen, eröffnen der Krankenhausführung nur beschränkte Rationalisierungspotentiale. ${ }^{40}$ Somit sind den Rationalisierungsbestrebungen im Krankenhaus natürliche Grenzen gesetzt. Ein Überschreiten dieser Grenzen könnte das eigentliche Ziel der Krankenhausarbeit gefährden. ${ }^{41}$

- Geringe Angebotselastizität: Bei kurzfristiger Änderung der Nachfrage erfolgt die Kapazitätsanpassung erst mit großer Verzögerung. Die Notwendigkeit, qualifizierte Fachkräfte für die Leistungserbringung im Krankenhaus einsetzen zu müssen, raubt dem Krankenhaus die Möglichkeit zur flexiblen Gestaltung der Leistungskapazität im kurzfristigen Bereich.

37 Vgl. Strehlau-Schwoll, H. (1993): Anpassung der Kosten- und Leistungsrechnung an die Erfordernisse des GSG-Deckungsbeitragsrechnung und relative Einzelkostenrechnung, in: Das Krankenhaus, 5/1993, S. 112.

${ }^{38}$ Vgl. Herder-Dorneich, P. (1985): Wettbewerb und Rationalitätsfalle im System der Gesetzlichen Krankenversicherung, in: Hamm, Neubauer (Hrsg,) 1985, S. 25.

39 Vgl. Unterhuber, H. (1986): Preissteuerung in der Krankenhausversorgung. Möglichkeiten und Grenzen der Anwendung von Preisen zur Steuerung der Versorgung mit Krankenhausleistungen, Dissertation an der Bundeswehruniversität München, 1986, S. 72.

${ }^{40}$ Vgl. Strehlau-Schwoll, H. (1993), S. 113.

${ }^{41}$ Vgl. Eichhorn, S. (1975), S. 14. 


\subsubsection{Aspekte der Wirtschaftlichkeit im Krankenhaus}

Das Wirtschaftlichkeitsprinzip als ein Ziel aller wirtschaftlichen Betriebe wird gemeinhin mit Rationalprinzip beschrieben, das zwei Varianten besitzt: ${ }^{42}$

i. Maximalprinzip: Dabei soll die größtmögliche Leistung mit gegebenen Mitteln realisiert werden.

ii. Minimalprinzip: Dabei soll eine bestimmte Leistung mit dem geringstmöglichen Mitteleinsatz erreicht werden.

Wenn auch beim Betrieb „Krankenhaus“ die Bedarfsdeckung an Krankenhausleistungen im Vordergrund steht, so orientieren sich doch alle Entscheidungen am Wirtschaftlichkeitsprinzip. In den Krankenhäusern greift dabei in der Regel Minimumprinzip, da die Ziele der Leistungserbringung festgelegt sind, aber die Mittel variiert werden können. Das bedeutet, die medizinisch notwendigen und zweckmäßigen Leistungen sollen mit möglichst geringen Kosten erbracht werden. ${ }^{43}$

Unter Wirtschaftlichkeit der Krankenhausversorgung versteht man die Angemessenheit von Art und Umfang der Arbeitsleistungen, Sachgüter und Betriebsmittel, die zur Erstellung der Einzelleistungen im Bereich von Diagnostik, Therapie, Pflege und Hotelversorgung eingesetzt werden (Mitteladäquanz des Leistungserstellungsprozesses). ${ }^{44}$

Bei der Bewertung der Wirtschaftlichkeit einer bedarfswirtschaftlich orientierten Unternehmung kommt der Frage, ob die von der Unternehmung erbrachten Leistungen wirtschaftlich erzeugt werden, die zentrale Bedeutung zu. Hierzu stellen sich eine Reihe von anschließenden Fragen: Wie kann man erkennen, ob ein Krankenhaus seine Leistungen wirtschaftlich erbringt? Was kostet die jeweilige

\footnotetext{
42 Vgl. Wöhe, G. (1990): Einführung in die Allgemeine Betriebswirtschaftslehre, 17. Aufl., München, 1990, S. 1.

43 Beide Prinzipien (Minimal- und Maximalprinzip) stellen auf eine optimale Relation von ZweckMittel ab, unterscheiden sich aber in ihrer Ausrichtung. die beiden Prinzipien können niemals gleichzeitig verwirklicht werden. Eine ständige Verringerung des Mitteeinsatzes führt jedoch zwangsläufig dazu, dass sich eine maximale Zweckerreichung nicht mehr erreichen läßt. Dies ist insbesondere der Fall bei Krankenhäuser in vielen Entwicklungsländern, die sehr begrenzten Ressourcen haben.

${ }^{44}$ Vgl. Hörmann, W., Ingruber, H. (1988) S. 29
} 
Leistung und könnten die gleichen Leistungen auch mit geringerem Aufwand erstellt werden?

Bei der Wirtschaftlichkeitsbewertung wird daher immer die relative Wirtschaftlichkeit eines Krankenhauses bzw. einer Fachabteilung betrachtet, d.h. der Vergleich mit vergleichbaren Krankenhäusern bzw. Fachabteilungen. Die Wirtschaftlichkeitsbewertung muss sich somit auf Betriebsvergleiche stützen. ${ }^{45}$ Die Beurteilung der Wirtschaftlichkeit nach betriebswirtschaftlichen Gesichtspunkten wird dabei exakterweise durch die Relation von Istkosten zu Sollkosten gemessen. ${ }^{46}$ Davon ausgehend müssen sich Überlegungen zur Beurteilung und Sicherung von Wirtschaftlichkeit instrumentell an folgendem prozessualen Ablauf von Information, Kontrolle und Rückkoppelung im Bereich des Krankenhausbetriebsprozesses orientieren: ${ }^{47}$

- Definition des Leistungszieles des Krankenhauses bzw. Dokumentation, Analyse und Beurteilung des Behandlungsergebnisses

- Aufstellen von Soll-Profilen für den Ablauf des Behandlungsergebnisses bzw. Dokumentation, Analyse und Beurteilung des Behandlungsergebnisses

- Aufstellen von Soll-Profilen für die Leistungsbereitschaft der Ressourcen bzw. Dokumentation der Ist-Leistungsbereitschaft, Analyse und Beurteilung der vorhandenen Ressourcen

- Aufstellen von Soll-Profilen für den Verbrauch an Produktionsfaktoren zur Erstellung der Sekundärleistungen bzw. Ermitteln des Verbrauchs an Produktionsfaktoren bei der Sekundärleistungserstellung, Analyse und Beurteilung des Leistungserstellungsprozesses.

Obwohl ein Krankenhaus nicht nach absolutem Wirtschaftlichkeits- und Marktprinzip arbeiten kann, ${ }^{48}$ ist aber heutzutage der Einsatz wirtschaftlicher und effek-

45 Vgl. Janssen, D. (1999): Wirtschaftlichkeitsbewertung von Krankenhäuser, Konzepte und Analysen von Betriebsvergleichen, Stuttgart, Berlin, Köln, 1999, S. 10.

${ }^{46}$ Keun, F. (1997) S.84.

47 Vgl. Hörmann, W., Ingruber, H. (1988) S. 18-3.

48 Die Verteilung der Krankenhausgüter auf die Bevölkerung hat nicht nach dem Kaufkraftprinzip, sondern nach dem Bedarfsprinzip zu erfolgen. Beispielsweise ist das einzelne Krankenhaus gezwungen, unabhängig vom Preis jeden Patienten aufzunehmen und im Rahmen seiner 
tiver Managementmethoden eine Überlebensfrage jedes Krankenhauses. Kein Krankenhaus kann es sich leisten, Fragen der Steuerung des aktuellen Leistungsgeschehens sowie der künftigen Leistungsstruktur dem Zufall zu überlassen. ${ }^{49}$ Die Notwendigkeit, dass Krankenhäuser eine strategische Planung betreiben müssen, ist unbestritten. Untersuchungsgegenstände der strategischen Planung sind die Organisationsstruktur, das Leistungsprogramm, der Ressourceneinsatz sowie die Unternehmens-/ Umweltbeziehungen. ${ }^{50}$

\subsubsection{Der Betriebsprozess des Krankenhauses}

Die spezifische Leistung des Krankenhausbetriebes ergibt sich aus dem Zusammenwirken der Elemente “Arbeitsleistungen“, „Sachgüter“" und „Betriebsmittel“, die durch die Krankenhausleitung sowie die zuständigen Organe des Krankenhausträgers als dem dispositiven Faktor geplant, organisiert und kontrolliert werden. $^{51}$ Zur Erbringung von Leistungen werden in verschiedenen organisatorischen Teilbereichen arbeitsteilig Ressourcen (Produktionsfaktoren) eingesetzt. Das wird schematisch in Abb. 6 dargestellt.

Leistungsfähigkeiten zu behandeln. Vgl. Siebig, J. (1993): Formen der flexiblen Budgetierung im Rahmen der Krankenhausfinanzierung, in: Das Krankenhaus, 2/ 1993, S. 92.

${ }^{49}$ Vgl. Conrad, H. J. (1997): Anforderung an ein leistungsfähiges Krankenhausmanagement, ProfitCenter- Steuerung durch dreistufige Deckungsbeitragsrechnung, in: DAS Krankenhaus, 10/ 1997, S. 607.

50 Vgl. Strehlau-Schwoll, H. (1996): Die Profit-Center Konzeption, Baustein der Führungsorganisation des Krankenhauses, in: F\&W, 4/ 1996,S. 317. 


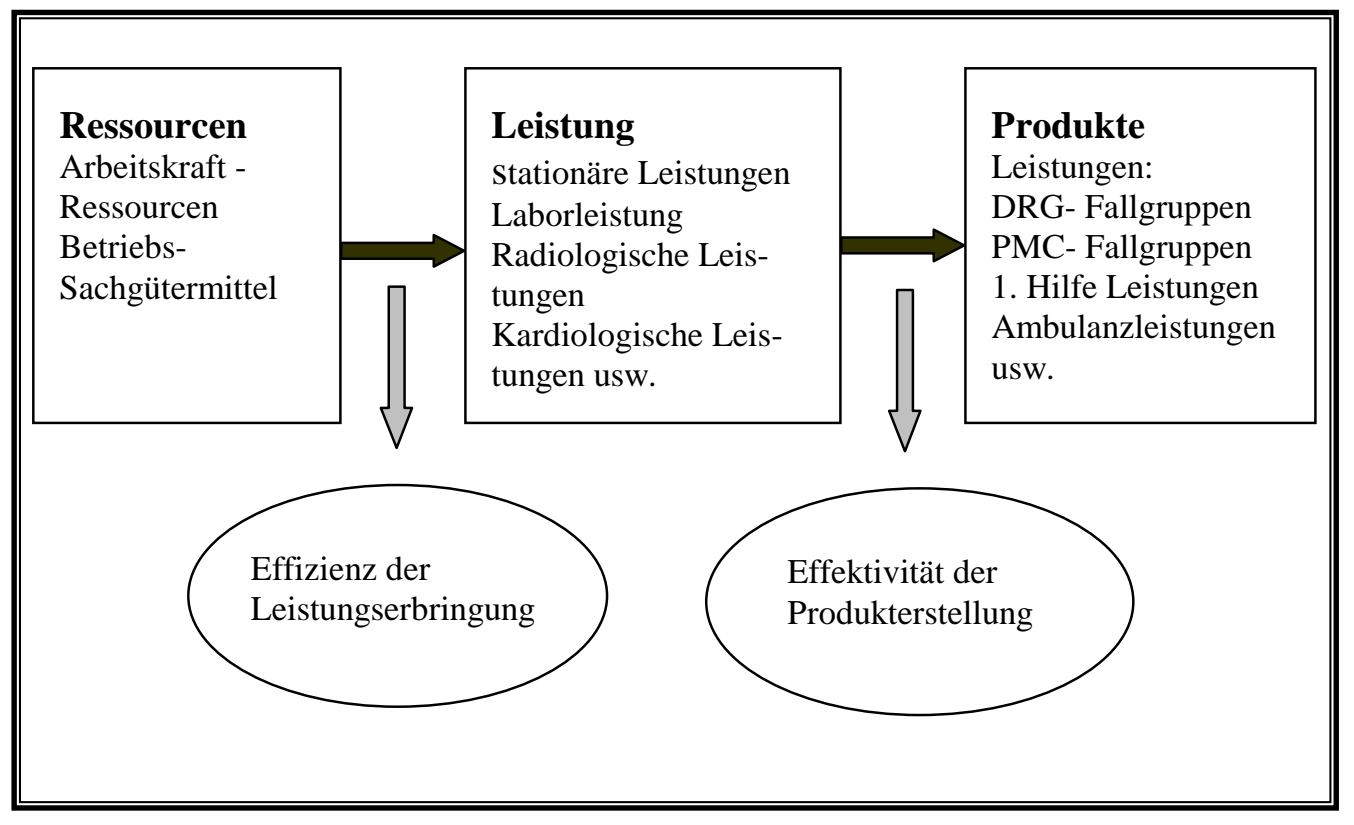

Abb. 6: Schematische Darstellung des Leistungsgeschehens im Krankenhaus . ${ }^{52}$

Der Produktionsprozess des Krankenhauses kann als ein mehrstufiger Prozess interpretiert werden, wobei der Primär-Output des Krankenhauses in der Veränderung des Gesundheitszustands des Patient zu sehen ist. Dies wird durch den Einsatz von Primär-Input bzw. Sekundär-Output, d.h. den verschiedenen Einzelleistungen der Diagnostik, Therapie, Pflege, Versorgung und Verwaltung bewirkt, die sich wiederum aus der Kombination von Produktionsfaktoren (Sekundär-Input) ergeben. ${ }^{53}$

Der Einsatz von Produktionsfaktoren wird auch als Input bezeichnet. Bei den Produktionsfaktoren unterscheidet man Potentialfaktoren und Repetierfaktoren. Produktionsfaktoren sind solche, die sich bei Erstellung der Krankenhausleistung nicht vermindern (z.B. menschliche Arbeitsleistung, Gebäude, Anlagegüter). Und Repetierfaktoren sind solche, die sich vermindern bzw. verbrauchen (z. B. Einmalmaterial, Lebensmittel).

Ist es noch ziemlich einfach, den Input des Betriebsprozesses des Krankenhauses als die Summe der eingesetzten Produktionsfaktoren zu abgrenzen, so ergeben sich

51 Vgl. Eichhorn, S. (1976): Krankenhausbetriebslehre - Theorie und Praxis des Krankenhausbetriebes, Band. II, 3. Aufl., Stuttgart, 1976, S. 7.

52 Quelle: Hildbrand, R. (1988): Kostenrechnung, in Handbuch Krankenhausrechnungswesen Grundlagen- Verfahren- Anwendungen, Eichhorn, S. (Hrsg.), 2. Aufl., Wiesbaden, 1988, S. 357. 
bei der Definition der Produktionsleistung „des Outputs“ im Unterschied zu einem Produktionsbetrieb größere Schwierigkeiten. Die heute noch übliche Messung der Krankenhausleistung als Zahl der geleisteten Pflegetage oder im Idealfall als Zahl der Einzelleistungen im diagnostisch- therapeutischen Bereich ist bei Einbeziehung des Patienten als Dienstleistungssubjekt unbefriedigend. Es geht nicht darum, eine bestimmte Anzahl von Leistungseinheiten $\mathrm{zu}$ produzieren, sondern den Gesundheitszustand des Patienten positiv zu beeinflussen. Dabei ergibt sich ein Veränderungswert aus dem Vergleich des Status des Patienten vom Anfang bis zum Ende des Krankenhausaufenthaltes.

Auf Grund dieser Betrachtungsweise ist nun der Primär-Output (die Primärleistung) des Krankenhausprozesses die Veränderung im Gesundheitszustand des Patienten (geheilt, gebessert, reaktiviert, rehabilitiert usw.). Gesamtwirtschaftlich gesehen kann dabei die Primärleistung des Krankenhauses auch als „Gesundheitskapitalbildung" gesehen werden.

Der Sekundär-Output setzt sich zusammen aus den Einzelleistungen im medizinischen Bereich. ${ }^{54}$ Dieser Sekundäroutput ist bei dieser Betrachtungsweise die Zahl der geleisteten Pflegetage, die Zahl der Einzelleistungen in Diagnose, Therapie, in Pflege und im Hotelbereich des Krankenhauses. Abb. 7 stellt die Betriebsprozess des Krankenhauses dar. ${ }^{55}$

Die Pflegetage und Behandlungsfälle werden in diesem Schema als Indikatoren verschiedener Typen von Zwischenpunkten verstanden, die unmittelbar unterhalb des eigentlichen Outputs „Gesunden“ anzusiedeln sind. ${ }^{56}$ Die Pflegetage stehen in diesem Kontext für die pflegerische Komponente, während die Behandlungsfälle die medizinische Komponente der Krankenhausleistungen darstellen. ${ }^{57}$

\footnotetext{
${ }^{53}$ Vgl. Eichhorn, S. (1979), S. 177.

54 Vgl. Steiner, P. (1997): Messung und Beurteilung öffentlicher Leistungen - Der Krankenhausoutput, Sternenfels, Berlin: Verl. Wiss. Und Praxis, 1997, S. 108.

${ }^{55}$ Vgl. Hörmann, W., Ingruber, H. (1988) S. 23.

56 Vgl. Preuß, O. (1994): Das Krankenhaus als Betrieb, in: Peters, S., Schär, W. (Hrsg.), Betriebswirtschaft und Management im Krankenhaus, S. 92 - 109, Berlin, 1994. S. 105.
} 


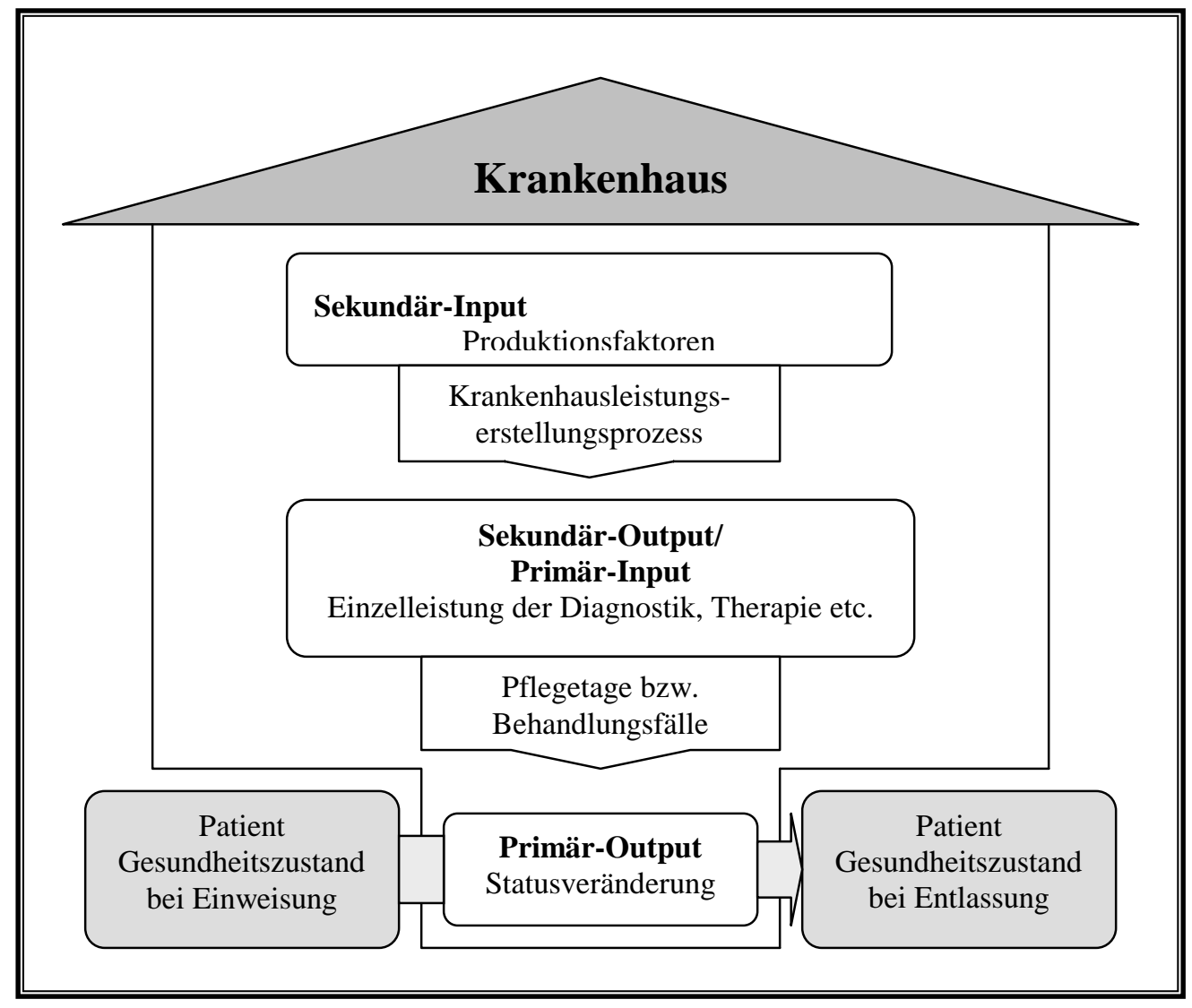

Abb. 7: Betriebsprozess des Krankenhauses ${ }^{58}$

\subsection{Krankenhäuser in Deutschland}

Der folgende Abschnitt betrachtet speziell die Situation der Krankenhäuser in Deutschland.

\subsubsection{Das Krankenhauswesen in Deutschland}

In der Bundesrepublik Deutschland ist das Krankenhauswesen eine öffentliche Aufgabe. Die Verpflichtung des Staates zur Schaffung und Erhaltung eines funktionierenden Krankenhauswesens folgt aus dem in Artikel 20 Abs. 1 des GrundHilfestellung in sozialen Notlagen als auch die Daseinsvorsorge im weitesten Sinne umfaßt. Dabei hat der Einzelne einen konkreten Rechtsanspruch auf staatliche Leistungen in dem hierunter $\mathrm{zu}$ subsumierenden Sektor des Krankenhauswesens. So ist aus den öffentlichen Aufgaben der

${ }^{57}$ Vgl. Andersen, H., Henke, K., Schulenberg, M. (1992): Basiswissen Gesundheitsökonomie, Band I, Einführende Texte, Berlin, 1992, S. 89.

58 Quelle: Schwartz, A. (1997), S. 17. 
Krankenhausversorgung eine verfassungsrechtlich verankerte Verpflichtung geworden, bei deren Erfüllung der Gesetzgeber Chancengleichheit für alle auf hohem Niveau postuliert. ${ }^{59}$

\subsubsection{Definition des deutschen Krankenhauses}

Die Definition eines Krankenhauses im deutschen Krankenhauswesen ist von den gesetzlichen Rahmenbedingungen geprägt. Das Krankenhaus wird als ein Dienstleistungsbetrieb besonderer Art definiert, der unter den Bedingungen des Krankenhausfinanzierunggesetzes (KHG) und der Bundespflegesatzverordnung das Ziel hat, Überschüsse zu erwirtschaften. ${ }^{60}$

Der Gesetzgeber beschreibt Krankenhäuser als „Einrichtungen, in denen durch ärztliche und pflegerische Hilfeleistung Krankheiten, Leiden oder Körperschäden festgestellt, geheilt oder gelindert werden sollen oder Geburtshilfe geleistet wird und in denen die zu versorgenden Personen untergebracht und verpflegt werden können“. ${ }^{61}$ Daneben dienen Krankenhäuser als Ausbildungsstätten für medizinisches und pflegerisches Personal. ${ }^{62}$ Die Krankenhäuser haben im gesundheitlichen Versorgungssystem eine zentrale Stellung mit vielfältigen Funktionen und Aufgaben. Sie sollen damit also

i. die Behandlung der einer Stationärenbehandlung bedürftigen Patienten, insbesondere der Schwerkranken und Schwerverletzten, nach Maßgabe des medizinischen Kenntnisstandes und unter Berücksichtigung des medizinischen Fortschritts ermöglichen,

ii. die Vorhaltung ausreichender Sach- und Personalressourcen gewährleisten, um jederzeit bei Unfällen, Notfällen, Katastrophen oder Seuchen die erforderliche medizinische Versorgung zu garantieren,

iii. eine humane ärztliche und krankenpflegerische Versorgung Sterbender bieten, für die das Krankenhaus oftmals letzte lindernde und sterbebegleitende $\mathrm{Zu}$ flucht ist,

\footnotetext{
${ }^{59}$ Vgl. Siebig, J. (1993), S. 92.

${ }^{60}$ Vgl. Gebhard, W. (1986): Controlling im Krankenhaus, in: Controlling 6/ 1986, S. 207.

${ }^{61} \mathrm{Vgl}$. 2 Abs. $1 \mathrm{KHG}$.

${ }^{62} \mathrm{Vgl}$ § 2 Abs. 1.a KHG.
} 
iv. die Entwicklung/Implementation des medizinischen, medizinischtechnischen, pharmakologischen und pflegerischen Fortschritts forcieren,

v. die Aus- und Weiterbildung der Ärzte, Pflegekräfte und anderer Gesundheitsberufe für das gesamte Gesundheitssystem sichern und

vi. Forschung und Lehre unterstützen. ${ }^{63}$

Wenn man sich in Deutschland anschaut, in welcher Trägerschaft sich deutsche Krankenhäuser befinden, trifft man auf drei unterschiedlichen Formen: öffentliche, freigemeinnützige und private Trägerschaften. ${ }^{64}$

Öffentliche Krankenhäuser in Deutschland sind solche, deren Trägerschaft eine kommunale Gebietskörperschaft, ein Bundesland, der Bund oder eine sonstige Körperschaft des Öffentlichen Rechtes ist. Im Einzelnen sind dabei zu unterscheiden: Bei den kommunalen Krankenhäusern die Gemeindekrankenhäuser, städtische Krankenhäuser, Kreiskrankenhäuser sowie Landes- bzw. Bezirkskrankenhäuser.

Freigemeinnützige Krankenhäuser werden von Trägern, die einer religiösen, humanitären oder sozialen Vereinigung zuzuordnen sind, auf der Grundlage der Freiwilligkeit und Gemeinnützigkeit unterhalten. Im Einzelnen gibt es z.B. Krankenhäuser der Kirchen, der einzelnen Kirchengemeinden, Ordenskrankenhäuser, Stiftungskrankenhäuser, Krankenhäuser des Roten-Kreuzes, Gemeinschaftskrankenhäuser oder Vereinskrankenhäuser. Hierbei ist in neuerer Zeit die Entwicklung zu beobachten, dass zunehmen die Krankenhäuser in $\mathrm{GmbH}$ mit einem gesamtverantwortlichen Geschäftsführer umgestellt werden. ${ }^{65}$

Private Krankenhäuser werden von ihren Trägern nach erwerbswirtschaftlichen Grundsätzen betrieben. Sie nennen sich zumeist Privatkrankenanstalten oder Privatkliniken.

\footnotetext{
${ }^{63}$ Vgl. Deutsche Krankenhausgesellschaft (1994 a): Positionen der DKG zur Reformdiskussion im Gesundheitswesen, Die Stellung des Krankenhauses in der künftigen gesundheitlichen Versorgung, in: Das Krankenhaus, 12/1994, S. 539.

${ }^{64}$ Vgl. Andersch, N. (1990): Krankenhausentwicklung und gewerkschaftliche Krankenhauspolitik: Eine Übersicht unter besonderer Berücksichtigung der ÖTV., Va\&g, Marburg, 1990, S. 17-19.

65 Dem hessischen Beispiel sind andere Bundesländer mit ihren Universitätsklinik gefolgt. Vgl. Schwarz, R. (1993), S. 116.
} 
Insgesamt gab es im Jahr 1998 in Deutschland 2.259 Krankenhäuser, die über 572.000 Krankenhausbetten verfügten. Für je 10.000 Einwohner standen 1998 durchschnittlich 69,7 Krankenhausbetten zur Verfügung (141 Einwohner pro Bett). ${ }^{66}$

\subsubsection{Die gesetzlichen Rahmenbedingungen für deutsche Krankenhäuser}

Die deutschen Krankenhäuser und somit das Krankenhauswesen unterliegen einer Fülle weiterer gesetzlicher Vorschriften, die sich im Lauf der Zeit mehrmals geändert haben. Heutzutage gelten u.a. die folgenden Gesetze und Regelungen:

- Gesetz zur wirtschaftlichen Regelung der Krankenhäuser und zur Regelung der Krankenhauspflegesätze (Krankenhausfinanzierungsgesetz - KHG): Das KHG verfolgt den Zweck, die Wirtschaftlichkeit der Krankenhäuser zu sichern, um eine bedarfsgerechte Versorgung der Bevölkerung mit leistungsfähigen, eigenverantwortlich wirtschaftenden Krankenhäusern zu gewährleisten und zu sozial tragbaren Pflegesätzen beizutragen. Das Gesetz ist 1972 verabschiedet worden, weil „ständige Defizite zu einer erheblichen Überalterung der Krankenhäuser geführt hatten und die Anforderungen einer ausreichenden Versorgung der Bevölkerung mit Krankenhausleistungen nicht mehr entsprachen“. ${ }^{67}$ Die Vorhaltung von Krankenhausleistungen wird als eine öffentliche Aufgabe verstanden. Nach dem KHG wird die Aufgabe erfüllt, indem Investitionskosten durch öffentliche Förderung übernommen werden. Die Finanzierung der laufenden Betriebs- und Behandlungskosten erfolgt dagegen über Pflegesätze, welche die Patienten bzw. ihre Krankenkassen zahlen. Die damit entstandene duale Krankenhaus-Finanzierung soll den Krankenhäusern einerseits die erforderlichen Investitions-mittel sichern und andererseits die Höhe der Pflegesätze mäßigen. ${ }^{68}$

\footnotetext{
${ }^{66}$ Vgl. Statistisches Bundesamt (1999 a): Mitteilung für Presse, Krankenhausstatistik 1998: Mehr Patienten, weniger Betten, kürzere Verweildauer, http://www.statistik-Bund.de/-presse/deutsch/pm/p9354094. htm, 01. 03. 2000, (Stand Am 14.01.1999).

${ }^{67}$ Schwarz, K. (1982): Auswirkung der Bevölkerungsentwicklung auf Leistungsnachfrage und Ausgaben im Gesundheitswesen, der Bundesminister für Arbeit und Sozialordnung (Hrsg.), Bonn, 1982, S. 1-10.

${ }^{68}$ Vgl. Hauschild, E., Schomacker, R., Strauf, H.G. (1981): Kriterienmodell zur Einordnung von Krankenhäusern in ein Abgestuftes Versorgungssystem, der Bundesminister für Arbeit und Sozialordnung (Hrsg.), Bonn, 1981, S. 9.
} 
- Das Fünfte Sozialgesetzbuch (SGB V): Es befasst sich mit der gesetzlichen Krankenversicherung. Es finden sich dort insbesondere Vorschriften, die den Leistungsanspruch das Versicherten und das Verhältnis von Krankenkassen zu Leistungserbringern, also auch zum Krankenhaus, regeln. Um das Ziel einer zentralstaatlichen Steuerung des Krankenhauswesens zu erreichen, werden vom zuständigen Bundesministerium gesetzliche Aktivitäten ausgelöst, die entscheidende Regelungen für den Krankenhausbereich vom zustimmungspflichtigen KHG zum SGB verlagern, bei dem die rechtliche Position und der Einfluss der Länder und Krankenhäuser ungleich schwächer ist. ${ }^{69}$

- Verordnung zur Regelung der Krankenhauspflegesätze (Bundespflegesatzverordnung BPflV): Es regelt das Vorgehensweise bei der Festlegung von Pflegesätzen.

- Das Gesundheitsstrukturgesetz GSG verfügt, dass spätestens seit 1. Januar 1996 die Betriebskosten mittels Abteilungsbudgets, Sonderentgelten und Fallpauschalen abgerechnet werden müssen. Das vormals bestehende Selbstkostendeckungsprinzip und die prospektive Budgetierung auf der Basis tagesgleicher Pflegesätze sind damit abgeschafft worden.

- Hierzu kommen diverse Gesetze, die Änderungen im SGB V und im KHG verfügten und zu Neufassungen der BPflV führten. Dazu gehören beispielsweise das Krankenhauskostendämpfungsgesetz vom 22. Dezember 1981, das Krankenhaus-Neuordnungsgesetz vom 20. Dezember 1984, das Gesundheitsreformgesetz (GRG) vom 20. Dezember 1988, das Gesundheitsstrukturgesetz (GSG) vom 21. Dezember 1992, das Stabilisierungsgesetz vom 29. April 1996, das 1. und 2 Krankenversicherung Neuordnungsgesetz (1. und 2. GKV-NOG) vom 12. Juni 1997 und schließlich Krankenversicherung-Solidaritätsgesetz (GKV-SolG) vom 28. Dezember 1998. ${ }^{70}$

\footnotetext{
69 Vgl. Bruckenberger, E. (1993): von der Länderkompetenz zum „Einkaufsmodell“ der Krankenkassen“ die Konsequenzen des Gesundheits-Strukturgesetzes für Krankenhäuser, in: das Krankenhaus, 2/ 1993, S. 77.

${ }^{70}$ Vgl. Trill, R. (2000): Krankenhaus-Management, Aktionsfelder und Erfolgspotentiale, 2. Erweiterte und überarbeitete Aufl. Neuwied, Kriftel: Luchterland, 2000, S. 47.
} 


\subsubsection{Wirtschaftlichkeit und Leistungsfähigkeit in deutschen Krankenhäusern}

Der Begriff der Wirtschaftlichkeit oder der sparsamen Wirtschaftsführung wird in allen das Krankenhaus direkt betreffenden Gesetzen und Verordnungen (KHG, BPflV sowie SGB V) mehrfach verwendet. Wenn auch der Begriff der Wirtschaftlichkeit zu den zentralen Begriffen der Krankenhausgesetzgebung zählt, besteht doch sein „Beitrag“ zur inhaltlichen Klärung des Wirtschaftlichkeitsbegriffs im Grunde nur in seiner wiederholten Erwähnung. Eine Definition seitens des Gesetzgebers erfolgte nicht. $^{71}$

Mit der Forderung nach einer leistungsgerechten Vergütung hat der deutsche Gesetzgeber auf Grund der neuen BPflV, die zum 01.01.1995 in Kraft trat, und deren Anwendung für alle Krankenhäuser ab 01.01.1996 zwingend war, verschiedene Arten von Krankenhausleistungen unterschieden, die auf einer unterschiedlichen Anspruchsgrundlage vergütet werden und für deren Kalkulation die einzubeziehenden Kosten festgelegt wurden.

Die nach $\S 1$ Abs. 1 BPflV zu vergütenden Krankenhausleistungen umfassen insbesondere die ärztliche Behandlung, die Krankenpflege, die notwendige Versorgung mit Arznei-, Heil- und Hilfsmitteln sowie die Unterbringung und Verpflegung der Patienten. ${ }^{72}$

Im Sinne der BPflV lassen sich Krankenhausleistungen in allgemeine Krankenhausleistungen und Wahlleistungen zerlegen: ${ }^{73}$

- Unter die allgemeinen Krankenhausleistungen werden dabei alle Krankenhausleistungen subsumiert, „die unter Berücksichtigung der Leistungsfähigkeit des Krankenhauses im Einzelfall nach Art und Schwere der Krankheit für die medizinisch zweckmäßige und ausreichende Versorgung des Patienten notwendig sind“. 74

- Wahlleistungen sind darüber hinaus erbrachte Krankenhausleistungen, deren Erbringung die allgemeinen Krankenhausleistungen nicht beeinträchtigen. Sie

\footnotetext{
${ }^{71}$ Vgl. Jansen, D. (1999), S. 10.

${ }^{72}$ Vgl. $\$ 2$ Abs. 1 BPflV.

${ }^{73}$ Vgl. $\$ 2$ Abs. 1 BPflV.

${ }^{74}$ Vgl. $§ 2$ Abs. 2 BPflV.
} 
umfassen etwa die Unterbringung in einem gesondert zu berechnenden Einzelzimmer oder die Behandlung durch einen liquidationsberechtigten Chefarzt. ${ }^{75}$

Der Umfang der Krankenhausleistungen bemisst sich nach den Erfordernissen des einzelnen Krankheitsfalls. Die Leistungen selbst müssen dem Wirtschaftlichkeitsgebot genügen. Sie müssen ausreichend, zweckmäßig und wirtschaftlich sein. Sie dürfen das Maß des Notwendigen nicht überschreiten. Leistungen, die nicht notwendig oder unwirtschaftlich sind, können Versicherte nicht beanspruchen, dürfen die Leistungserbringer nicht bewirken und die Krankenkassen nicht bewilligen. ${ }^{76}$

Der deutsche Gesetzgeber hat mit einer Reihe von Festlegungen die Krankenhäuser darauf verpflichtet, Transparenz in die Qualität ihrer Leistungsprozesse sowie in die Effizienz ihrer Kostenstrukturen zu bringen, um medizinische Versorgungsqualität sicherzustellen. Gesetzliche Einzelparagraphen, die sich auf die Leistungsfähigkeit und Wirtschaftlichkeit des Krankenhauses beziehen, sind unter anderem $\S 26$ BPflV sowie $\S \S 70,109,112,113,137,301$ SGB V.

Um die tatsächliche Leistungsfähigkeit eines Krankenhauses im Hinblick auf die gesetzlichen Anforderungen zu sichern, müssten folgende Fragen gestellt werden: ${ }^{77}$

- Wie organisiert und steuert ein Krankenhaus den reibungslosen Übergang eines Patienten von einer Versorgungsphase in die nächste (§§ 112, 301 SGB V)?

- Durch welche Organisations- und Kooperationsformen verzahnt das Krankenhaus den ambulanten mit dem stationären Sektor, und welche Wirkungen bzw. Kosten und Patientenzufriedenheit sind damit verbunden (§§ 39. 115 a, b SGB V)?

- Inwieweit ist das Krankenhaus fähig, die gesundheitspolitischen Ziele der Landesregierung zu stützen?

- In welcher Form stellt das Krankenhaus eine ganzheitliche Betreuung des Patienten im Rahmen eines regionalen Gesundheitsnetzwerkes sicher?

\footnotetext{
${ }^{75}$ Vgl. § 22 BPflV.

${ }^{76}$ Vgl. § 12 Abs. 1 SGB V.

${ }^{77}$ Vgl. Von Eiff, W. (1997): Krankenhausbetriebsvergleich, Controllinginstrument zur Planung von Leistungsprozessen, in: das Krankenhaus, 10/ 1997, S. 613-614.
} 
Gesetzlich sollen Krankenhäuser sich an Maßnahmen zur Qualitätssicherung beteiligen, um die Qualität der Leistungen zu sichern. ${ }^{78}$ Entsprechend erstellen die Bundesländer Krankenhauspläne und Investitionsprogramme ${ }^{79}$ und haben Krankenhäuser einen Anspruch auf Förderung durch das Land, sofern sie in den Krankenhausplan aufgenommen und im Investitionsprogramm berücksichtigt worden sind. ${ }^{80}$ Die Länder legen somit fest welches Ausmaß das durch sie mitzufinanzierende Angebot von Krankenhausleistungen annehmen soll bzw. darf. Die Landesverbände der Krankenkassen, die Verbände der Ersatzkassen und der Landesausschuss des Verbandes der privaten Krankenversicherung können gemeinsam die Wirtschaftlichkeit, Leistungsfähigkeit und Qualität der Krankenhausleistung prüfen lassen. Sie bestellen dazu einvernehmlich mit dem Krankenhaus einen Prüfer. ${ }^{81}$ Die von einem Krankenhaus angebotenen Wahlleistungen muß der Patient entweder selbst oder durch eine freiwillige Zusatzversicherung finanzieren.

\subsubsection{Finanzierungs- und Entgeltsystem der deutschen Krankenhäuser}

\subsubsection{Das Finanzierungssystem}

Das Finanzierungssystem der deutschen Krankenhäuser ist als dualistisches System aufgebaut. ${ }^{82}$ Hierbei stammen in Gegensatz zur monistischen Finanzierung die Finanzmittel aus zwei unterschiedlichen Quellen, die jeweils nur für abgegrenzte Kostenbereiche gelten. ${ }^{83}$ Die Investitionskosten werden dabei im Zuge öffentlicher Förderung von den betreffenden Bundesländer und Kommunen übernommen, wogegen die laufenden Betriebskosten von Krankenkassen bzw. den Beitragszahlern erstattet werden.

Betriebskosten sind alle Kosten, die dem Krankenhaus durch die Patientenversorgung direkt, aber auch indirekt durch die Aufrechterhaltung der Betriebsbereit-

\footnotetext{
${ }^{78}$ Vgl. $\$ 137$ SGB V.

${ }^{79} \mathrm{Vgl}$. $8 \mathrm{KHG}$.

${ }^{80} \mathrm{Vgl}$ \& $8 \mathrm{KHG}$.

${ }^{81}$ Vgl. $§ 113,1$ SGB V.

82 duale oder dualistische Finanzierung, da ein Teil der Ausgaben über die Länder, der andere Teil u.a. über den Pflegesatz abgedeckt werden muss.

${ }^{83}$ Vgl. Raem, A. (1996): der Arzt als Manager, München, 1996, S. 27.
} 
schaft in medizinischer, technischer und administrativer Notwendigkeit entstehen. Diese Kosten umfassen alle Kosten, die für eine laufende Erbringung der stationären Krankenhausleistungen aufgewendet werden müssen. Darin enthalten sind v.a. Personalkosten, Sachkosten und die Instandhaltungskosten. ${ }^{84}$

Investitionsgüter und damit verbundene Abschreibungen finden dagegen keinen Eingang in die Betriebskosten, da sie aus Fördermitteln des Staates durch Steuereinnahmen finanziert werden. ${ }^{85}$ In Abb. 8 wird das System der Krankenhausfinanzierung in Deutschland dargestellt.

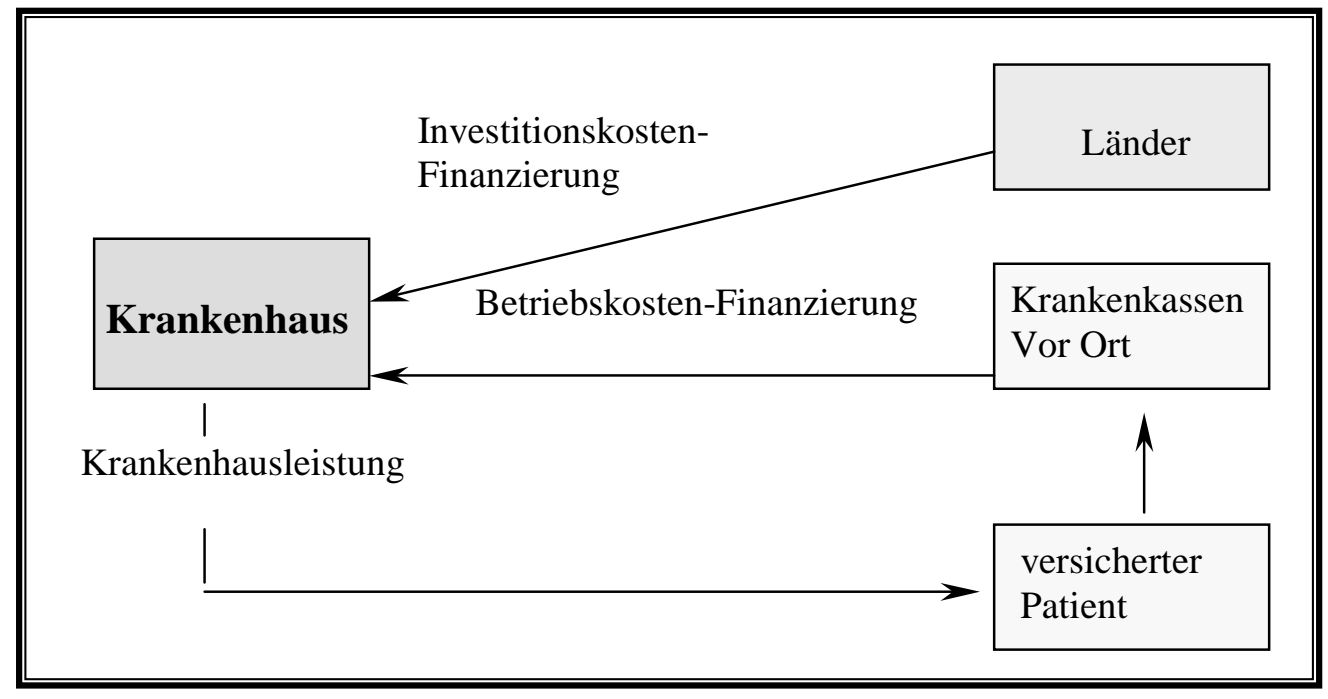

Abb. 8: Finanzierungssystem des deutschen Krankenhauses ${ }^{86}$

\subsubsection{Das Entgeltsystem in deutschen Krankenhäusern}

Der Einstieg in ein leistungsorientiertes Entgeltsystem erfolgt schrittweise, wobei „künftig nicht mehr das belegte Bett, sondern definierte Behandlungsleistungen die

\footnotetext{
${ }^{84}$ Vgl. Jungmann- Ginkel, E., Kober, K. (1993): Krankenhausbetriebslehre für Führungskräfte der Krankenpflege, Lorsch, 1993, S. 117.

${ }^{85}$ Vgl. ebenda, S.23.

${ }^{86}$ Quelle: Haubrock, M. (1994), S. 218.
} 
Bezugsbasis der Vergütung sein sollen“. ${ }^{87}$ Das Krankenhaus in Deutschland ist somit nicht mehr als „Einproduktunternehmen“ $\mathrm{zu}$ sehen, das nur Pflegetage „erstellt“, sondern als ein Unternehmen, das vielfältige Produkte anbietet.

Allgemeine Krankenhausleistungen werden vergütet durch:

- Pauschalierte Entgelte nach § 11 BPflV (Fallpauschalen und Sonderentgelt)

- Einen Gesamtbetrag nach $\S 11$ BPflV (Krankenhaus-Individuell-Restbudget), der über tagesgleiche Pflegesätze nach § 13 BPflV (Abteilungspflegesätze, Basispflegesatz) den Patienten oder ihre Kostenträgern (Krankenkassen oder sonstige Einrichtungen, die die Kosten der ambulanten Behandlungen tragen) anteilig berechnet wird. ${ }^{88}$ Abb. 9 stellt das Vergütungssystem der deutschen Krankenhäuser dar:

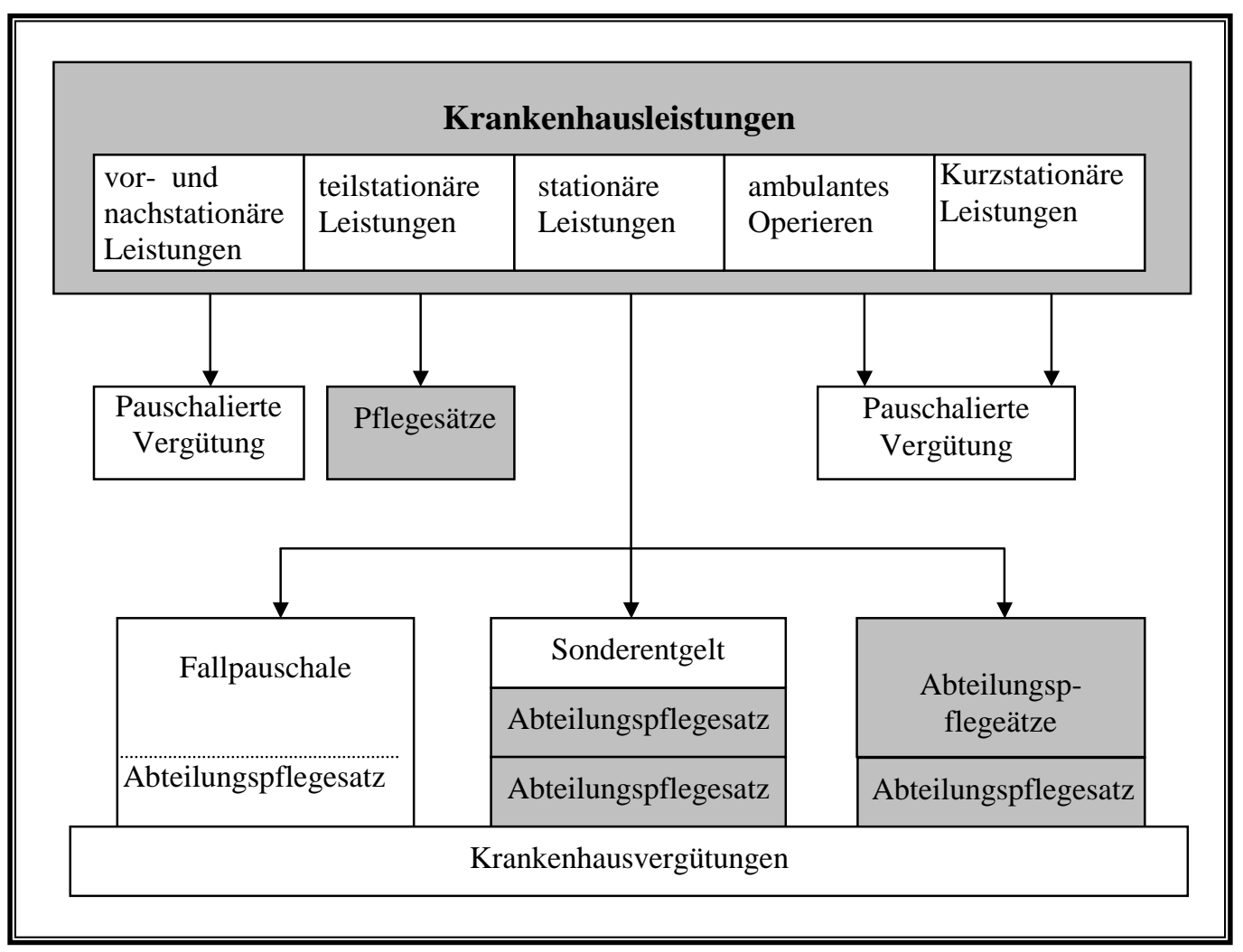

87 Neubauer, G., Zelle, B. (1996): Fallpauschalen: Ein Ansatz zu einer Leistungsbezogenen Krankenhausvergütung, in: Adam, D. (hrsg.): Krankenhausmanagement, Schriftenreihe zur Unternehmensführung (SzU.), Band 59, Wiesbaden 1996, S. 27.

${ }^{88}$ Vgl. 4.2.3.1. 
Abb. 9: Vergütungssystem von Krankenhausleistungen der deutschen Krankenhäuser ${ }^{89}$

Mit den Fallpauschalen werden die gesamten allgemeinen Krankenhausleistungen für die Behandlung eines Patienten, der einer nach Therapie und Diagnose abgrenzbaren Gruppe angehört, vergütet. In Anlage $1 \mathrm{zu} \S 11 \mathrm{BPflV}$ findet sich ein Katalog von Krankenhausleistungen, die derzeit durch Fallpauschalen vergütet werden. Bei dieser Vergütungsform spielt die tatsächliche Verweildauer eines Patienten im Krankenhaus grundsätzlich keine Rolle. Für sogenannte Ausreißerpatienten, die wesentlich länger behandelt werden müssen, als in der Fallpauschale berücksichtigt, ermöglicht $§ 14$ Abs. 7 BPflV innerhalb der Grenzverweildauer die zusätzliche Abrechnung von tagesgleichen Pflegesätzen. ${ }^{90}$ Der Fallpauschalenpatient wird nach Überschreiten der Grenzverweildauer $\mathrm{zu}$ einem „neuen“ Budgetpatienten. ${ }^{91}$

Mit dem Sonderentgelt wird ein Teil der allgemeinen Krankenhausleistungen für einen bestimmten Behandlungsfall vergütet, im Wesentlichen operative Leistungen sowie die Kosten von Implantaten, Transplantaten und des medizinischen Sachbedarfs. Anlage 2 zu $§ 11$ BPflV enthält einen Katalog der Behandlungsfälle, für die ein Sonderentgelt in Rechnung gestellt werden kann. Die sonstigen ärztlichen und pflegerischen Dienste, die lediglich mit einer Schlüsselung der eigentlichen Leistung zurechenbar sind, bleiben innerhalb eines Sonderentgeltes unberücksichtigt. ${ }^{92}$ Daher stellt eine Kombination der Sonderentgelte ein Teilmodul der Fallpauschale dar. ${ }^{93}$ Für jede Fallpauschale existiert ein korrespondierendes Sonderentgelt, wobei der Umkehrschluss nicht gilt. Kann für einen Behandlungsfall

\footnotetext{
${ }^{89}$ Quelle: Neubauer, G. (1999): Formen der Vergütung von Krankenhäusern und deren Weiterentwicklung, in: Braun, G. (Hrsg.), Handbuch Krankenhausmanagement, Baustein für eine moderne Krankenhausführung, Stuttgart, 1999, S. 28.

90 Vgl. Schmidt-Rettig, B. (1995): Vom selbstkostendeckenden Pflegesatz zu fallpauschalierten Preisen, in: Eichhorn, S., Schmidt-Rettig, B. (Hrsg.): Krankenhausmanagement im Werte- und Strukturwandel, Stuttgart, Berlin, Köln, 1995, S. 140.

$91 \S 14$ Abs. 7 BPflV.

92 Vgl. Tuschen, K.H., Philippi, M. (1995): Leistung- und Kalkulationsaufstellung im Entgeltsystem der Krankenhäuser, Stuttgart, Berlin, Köln, 1995, S. 5.

${ }^{93}$ Vgl. Bundesministerium für Gesundheit (1995 a): Leitfaden zur Einführung von Fallpauschalen und Sonderentgelten gemäß BPflV 1995: Datenbedarf, Kalkulationsgrundlagen, Abrechnungsmodalitäten, Kostengliederung, Schriftenreihe des Bundesministeriums für Gesundheit, Band 44, Baaden-Baaden, 1995, S. 3.
} 
sowohl eine Fallpauschale als auch ein Sonderentgelt in Ansatz gebracht werden, hat grundsätzlich die Einordnung als Fallpauschale Vorrang. ${ }^{94}$ Die zusätzliche Einführung von Sonderentgelten soll es ermöglichen, sehr kostenintensive Behandlungsfälle, die in keine Fallpauschalenkategorie fallen, wenigstens teilweise über pauschalierte Festpreise leistungsgerecht zu vergüten. ${ }^{95}$

Die genannten Kataloge enthalten keine Preise, sondern Punktzahlen i.S.v. Bewertungsrelationen, die bundeseinheitlich festgelegt sind. Der tatsächlich in Form einer Fallpauschale oder eines Sonderentgelts zu zahlende Preis ergibt sich durch die Multiplikation dieser Punktzahlen mit einem von den Verbänden der Krankenhäuser und Krankenkassen jeweils auf Landesebene unter Beachtung einer bedarfsgerechten medizinischen Versorgung der Bevölkerung festzulegenden Punktwert.

Mit der Einführen von Festpreisen entstehen für Krankenhäuser konkrete Anreize, die Kosten pro Behandlungsfall so gering wie möglich zu halten, indem Einsparungspotentiale identifiziert werden und durch Leistungsausweitungen Degressionseffekte einsetzen können. Da dem Krankenhaus unabhängig von der Verweildauer ein konstanter Erlös gegenübersteht, ist es nicht mehr sinnvoll, ein Bett möglichst lange mit einem Patienten zu belegen. Kurze Verweildauer und viele Fälle pro Bett verbessern die Erfolgslage, indem die durchschnittlichen Behandlungskosten pro Fall sinken. ${ }^{96}$ Dies ist in der Praxis der deutschen Krankenhäuser der Fall, wobei die durchschnittliche Verweildauer der Patienten im Krankenhaus von 11,4 Tagen 1996 auf 11 Tage 1997 und auf 10,7 Tage 1998 sanken. $^{97}$

Der Anteil der derzeit über Fallpauschalen und Sonderentgelte vergüteten Leistungen wird bei etwa $30 \%$ der Vergütung des gesamten operativen Leistungsspektrums bzw. bei etwa $20 \%$ der gesamten Kosten eines Krankenhauses liegen. Durch die beabsichtigte Festlegung weiterer Fallpauschalen und Sonderentgelte

\footnotetext{
94 Vgl. § 14 BPflV.

95 Vgl. Pfaff, M. (1995): Das Krankenhaus im Gefolge des Gesundheitsstrukturgesetzes 1993, Baden- Baden, 1995, S. 91.

96 Vgl. Neubauer, M., Breu, M. (1999): Budgetäre Auswirkungen von Verweildauerreduktionen, Ressourceneinsparung durch Verweildauerreduktion, eröffnen Entscheidungs- und Handlungsoptionen, in: F\&W, 2/1999, 112-113.
} 
wird dieser Anteil wachsen, so dass die Steuerungswirkung der neuen Entgeltformen im Zeitablauf mehr Gewicht erhalten wird. ${ }^{98}$

Der verbleibende Teil der Kosten der allgemeinen Krankenhausleistungen, der nicht durch Fallpauschalen oder Sonderentgelte vergütet wird, wird gemäß $§ 12$ BPflV über ein kostenorientiertes, zwischen Krankenhaus und Krankenkassen auszuhandelndes, flexibles Budget erstattet, wobei erstattet i.S.v. ,,angemessen vergütet" zu verstehen ist. Dieses Budget wird nach $\S 13$ BPflV den Patienten bzw. deren Krankenkassen in Form von Abschlagszahlungen, die sich durch Summation differenzierter tagesgleicher Pflegesätze ergeben, in Rechnung gestellt. Die im Budget vereinbarten Erlöse für unmittelbar oder mittelbar erbrachte ärztliche oder pflegerische Leistungen verschiedener Krankenhausbereiche werden über spezifische Abteilungspflegesätze, der dann noch im Restbudget verbleibende Teil der allgemeinen Krankenhausleistungen, z.B. für Unterkunft und Verpflegung (Hotelkosten) oder Verwaltung, ${ }^{99}$ über einen einheitlichen Pflegesatz, den Basispflegesatz, vergütet. Die Pflegesätze orientieren sich an den Pflege- und Berechnungstagen, die in einem Krankenhaus anfallen. Allerdings handelt es sich nach neuer Regelung um ein System von nach verschiedenen Bereichen differenzierten Pflegesätzen, die zudem auf einer niedrigeren Bemessungsgrundlage basieren, da die durch Fallpauschalen und Sonderentgelte vergüteten Leistungen gesondert betrachtet werden. ${ }^{100}$

Mit der Einführung des Budgets bzw. der daraus abgeleiteten Pflegesätze wird dem jeweiligen Krankenhaus bei wirtschaftlicher Betriebsführung die Erfüllung seines spezifischen Versorgungsauftrags ermöglicht. Mit der Einführung der gesonderten Abrechnung der Basisleistungen wurde vor allem das Ziel verfolgt, die Kostenstruktur der Krankenhäuser transparenter $\mathrm{zu}$ machen und die inner-

\footnotetext{
97 Vgl. Statistisches Bundesamt (1998): Mitteilung für Presse, Erneute Zunahme der Zahl der Patienten in den Krankenhäusern, http://www.statistik-Bund.de/presse/deutsch/pm/p9354094. htm, 01.03.2000, (Stand Am 22.09.1998), S. 1.

98 Vgl. Strehlau- Schwoll, H. (1997): Kostenrechnung und Kostenmanagement im Krankenhaus, in: Freidank, C., Götze, U., Huch, B., Lueter, G., (Hrsg.): Kostenmanagement, Heidelberg, 1997, S. 538.

${ }^{99}$ Vgl. § 11 BPflV.

${ }^{100}$ Vgl. Strehlau- Schwoll, H. (1997), S. 541.
} 
betriebliche Verrechnung über Schlüsselgrößen zwischen Abteilungen zu vermeiden. ${ }^{101}$

Auch kann der externe Wirtschaftlichkeitsvergleich mit Krankenhäusern ähnlicher Größenordnung detaillierter durchgeführt werden, als dies bisher mit einem einheitlichen Pflegesatz möglich war. ${ }^{102}$

\section{3 Das syrische Krankenhaus als wirtschaftlichen Betrieb}

Dieser Abschnitt wird in Abgrenzung zur Darstellung der Situation Deutschland die Situation der syrischen Krankenhäuser näher betrachten.

\section{3.1 Allgemeine Betrachtungen der syrischen Krankenhäuser}

Es gibt in Syrien keine spezielle Krankenhauslehre, die das Krankenhaus als wirtschaftlichen Betrieb behandelt. Überhaupt ist das Thema „Krankenhaus als wirtschaftlicher Betrieb“ ein neues Thema, das erst im letzten Jahrzehnt in Syrien sowohl in der Wirtschaftslehre als auch in den an der für Betriebsführung orientierten rechtlichen Rahmenbedingungen betrachtet wurde.

Bisher wurden im syrischen Krankenhauswesen lediglich die medizinischen und sozialen Schwerpunkte behandelt. Über die wirtschaftlichen Aspekte gibt es fast keine wissenschaftlichen Studien. Ursachen könnten dabei sein, dass die Entwicklungsstufe der syrischen Wirtschaft sich auf die landwirtschaftlichen und teilweise auf die industriellen Betriebe konzentriert, da die Dienstleistungsbetriebe relativ neu waren. Insbesondere der Krankenhausbetrieb ist ganz neu vom wirtschaftlichen Gesichtspunkt wissenschaftlich betrachtet worden, obwohl das erste Krankenhaus in den arabischen Ländern in Syrien im Jahr 706 begründet wurde. ${ }^{103}$

In der Gesetzgebung gibt es auch kein gesetzlichen Vorschriften, die den Krankenhausbetrieb regeln. Als gesetzliche Rahmenbedingungen gelten allgemein Handels- und Sozialgesetze an den Krankenhäusern, die auch die anderen Handelsund Industriebetriebe regeln. Um die Besonderheiten der Krankenhäuser betrachten zu können, wird bei der Gründung eines Krankenhauses ein Krankenhaus-

\footnotetext{
$\overline{101}$ Vgl. Tuschen, K.H., Philippi, M. (1995), S. 9.

102 Vgl. Neumann, U. (1999): Der leistungsorientierte Krankenhausbetriebsvergleich, in: F\&W, 5/1999, S. 426- 247.
} 
Handbuch eingerichtet werden, das von dem Gesundheitsministerium an Hand der allgemeinen rechtlichen Rahmenbedingungen akzeptiert werden. Jedes Krankenhaus-Handbuch sollte ausreichende Verordnungen über Begriffsdefinitionen, Zielsysteme, Organisationsordnung usw. umfassen. Darüber hinaus soll jedes Krankenhaus die häufig von der Regierung erlassenen Anordnungen befolgen, die die detaillierten durchgeführten Aktivitäten regelt. Dies gilt sowohl für staatlichen als auch für privaten Krankenhäusern.

\subsubsection{Die Begriffsdefinition des syrischen Krankenhauses}

Da es weder ein Krankenhauswesen noch Gesetze bezogen auf Krankenhäuser gibt, findet man keine Begriffsdefinition, die ein Krankenhaus als wirtschaftlichen Betrieb definiert. Die einzige Möglichkeit, einen Krankenhausbetrieb in Syrien zu determinieren, steht durch das Krankenhaus-Handbuch jedes Krankenhaus zu verfügen. ${ }^{104}$ Das Handbuch des Uni-Klinikums Damaskus hat das Krankenhaus als staatliche Gesellschaft definiert, die eine juristische Person mit wirtschaftlicher und administrativer Eigenständigkeit darstellt. ${ }^{105}$ Demnach hat das Krankenhaus die Aufgabe:

1. gemeinsam mit anderen medizinischen Organisationen den medizinischen und pflegerischen Bedarf in der arabischen Republik Syrien zu decken,

2. die medizinische Fakultät und Institutionen der Universität Damaskus mit Studiums- und Praktikumbedarf zu versorgen sowie

3. wissenschaftliche Untersuchungen in Verbindung mit dem „Hoch Studium Ministerium“" auszuführen. ${ }^{106}$

Als Krankenhausarten unterscheidet man in Syrien zwei Arten: staatliche ${ }^{107}$ und private Krankenhäuser. Die staatlichen Krankenhäuser haben hinsichtlich der Größe und Leistungskapazität eine große Bedeutung. Aber auch in den letzten

103 Farruch, O. (1970 Arb.): die Geschichte der Wissenschaften bei dem Arabern. Dar Alielm lemalaien, Beirut, 1970, S. 291.

${ }^{104}$ Harstanie, H. (1990 Arb.): Krankenhaus-Management, Institut für Management (Hrsg.), Read, Saudiareiben, 1990, S. 453.

105 § 1 Das Handbuch des Klinikums „Assad“ der Universität Damaskus.

106 § 2 Das Handbuch des Klinikums „Assad“ der Universität Damaskus. 
Jahren mit der Wirtschaftsliberalisierung sind private Krankenhäuser relevante Unternehmen geworden.

In Syrien gab es 1998 insgesamt 353 Krankenhäuser mit 18.737 Krankenhausbetten. Dabei hatten die 65 staatliche Krankenhäuser 13.337 Betten und die 285 private Krankenhäuser 4.411 Betten. Damit standen 1998 für je 10.000 Einwohner durchschnittlich 12,9 Krankenhausbetten zur Verfügung (775 Einwohner pro Bett). ${ }^{108}$

\subsubsection{Wirtschaftlichkeit und Leistungsfähigkeit in syrischen Krankenhäusern}

Generell hat die Wirtschaftlichkeit in vielen Ländern, die ihre Wirtschaft sozialistisch führt, eine geringe Rolle in der Betriebsführung gespielt. Dies war ebenfalls in Syrien als einem Land der Fall, das eine sozialistische Tendenz in der Wirtschaftsführung hat. ${ }^{109}$ Die meisten Betriebe haben wenig nach dem Marktprinzip gearbeitet, wodurch die Wirtschaftlichkeit von geringer Bedeutung war.

In den letzten Jahren wurde die Wirtschaft in Syrien jedoch nachhaltig reformiert. Dabei wurde die gesamte Wirtschaft liberalisiert und die Privatwirtschaft weiter und stärker neben der staatlichen Wirtschaft gefördert. ${ }^{110}$ Eine steigende Tendenz zur Orientierung am Marktprinzip und am betriebswirtschaftlichen Management in der Betriebsführung sind zu sehen, dass die Wirtschaftlichkeit mehr und mehr ins Blickfeld rückt.

Zur Beurteilung der Wirtschaftlichkeit in den syrischen Krankenhäusern muss zwischen privaten und staatlichen Krankenhäusern differenziert werden.

1. Wirtschaftlichkeit in privaten Krankenhäusern: Da das primäre Ziel der syrischen Privat-Krankenhäuser als erwerbwirtschaftliche Betriebe die Erzie-

107 Träger eines staatlichen Krankenhauses in Syrien sind u. a. das Gesundheitsministerium, eine von den vier syrischen Universitäten und das Verteidigungsministerium.

108 Syrische zentrale Statistikamt: Jahresbericht 1998, Damaskus, 1999, S.387.

109 In Syrien war der Wirtschaft nicht absolut sozialistisch, da es immer Privatwirtschaft und Gemischtwirtschaft neben der stattlichen Wirtschaft gab. Aber die großen und wichtigen Betriebe waren staatlich, die Regierung hatte der staatlichen Wirtschaft als Leitungssektor. Vgl. Poelling, S. (1996): The Role of the Private Sector in the Syrian Economy, in: Economic Liberalization and Privatization in Socialist Arab Countries,: Hopfinger, H., (Hrsg.), Gotha: Perthes, 1996. S. 155-157.

110 Sukkar, N. (1996): Economic Liberalization in Syria, in: Economic Liberalization and Privatization in Socialist Arab Countries, Hopfinger, H., (Edit.), Gotha, Perthes, 1996,147-148. 
lung von Einkommen und Gewinn ist, arbeiten sie nach dem absoluten Wirtschaftlichkeits- und Marktprinzip. Der in der Praxis von "KrankenhausMarkt" bestimmte Preis soll alle aufgebrachten Kosten decken sowie möglichst hohe Gewinne erzielen. Dagegen wird in manchen Behandlungsfällen, bei denen der Preis vom staatlichen zuständigen Ministerium für Gesundheit oder von der Ärztekammer fest gelegt wird, die Wirtschaftlichkeit in den syrischen PrivatKrankenhäusern nach Maximal-Prinzip durchgeführt, d.h. einem Patienten werden nur diejenige Krankenhaus-Leistungen gegeben, deren Kosten mit dem von Krankenhaus erwartenden Gewinn den festen Preis nicht überschreiten.

2. Wirtschaftlichkeit in staatlichen Krankenhäusern: Bei diesen Krankenhäusern als bedarfswirtschaftliche Betriebe ist eine Erzielung von Einkommen oder Gewinn nicht festgesetzt, sondern die Deckung des Gesundheitsbedarfs der Bevölkerung. Somit ist hier die Wirtschaftlichkeit nicht vorgesehen. Fast alle Leistungen der staatlichen Krankenhäuser sind für Patienten kostenlos, und es gibt kaum andere Kostenträger wie z. B. Krankenkassen, die die Kosten dem Krankenhaus erstatten. Aber mit der Liberalisierung der Wirtschaft und Reformierung der stattlichen Betriebe ist der Einsatz von Ressourcen und Planung der Leistungsprogramme nach wirtschaftlichen und effektiven Managementmethoden von großer Bedeutung geworden.

\subsubsection{Das Finanzierungssystem in den syrischen Krankenhäusern}

Die Finanzierung der syrischen Krankenhäuser unterscheidet sich auch bei privaten und staatlichen Krankenhäusern. Das Finanzierungssystem der privaten Krankenhäuser ist in Abb. 10 dargestellt: 


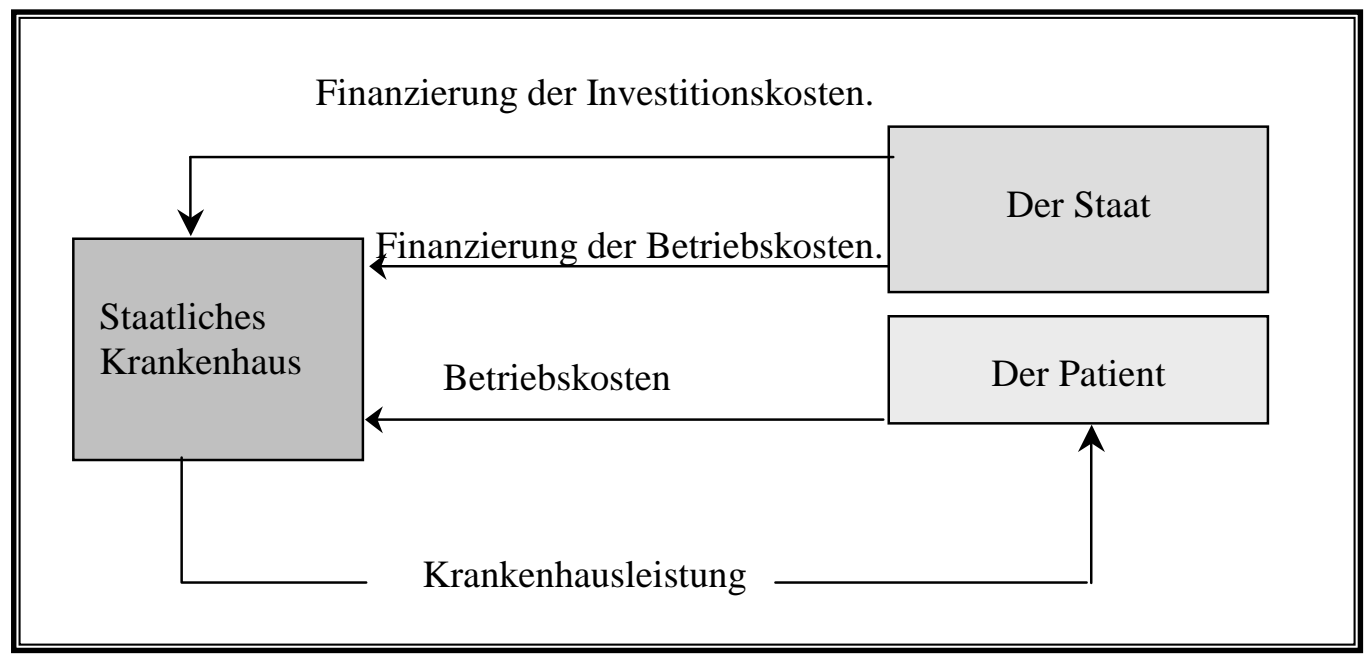

Abb. 10: Finanzierungssystem der privaten Krankenhäuser in Syrien

Ein Privat-Krankenhaus wird in Form einer KG oder GmbH von privatem Kapital finanziert, womit die gesamten Kosten von dem betreffenden Patienten selbst erstattet werden. Dem versicherten Patient können diese Kosten dann von seiner Krankenkasse gänzlich oder anteilig ausgeglichen werden.

Anders ist das Finanzierungssystem bei staatlichen Krankenhäusern, das in Abb. 11 dargestellt.

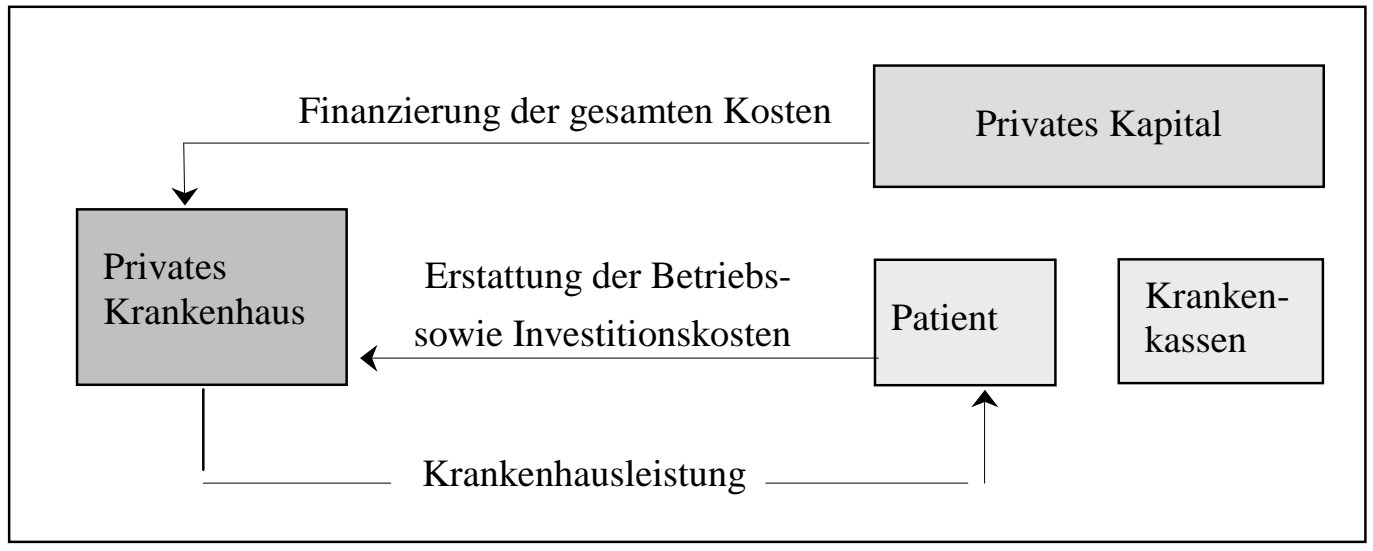

Abb. 11: Finanzierungssystem der staatlichen Krankenhäuser in Syrien. 
Hierbei werden die gesamten Kosten der staatlichen Krankenhäuser von der Regierung finanziert. Und da deren Leistungen kostenlos sind, wird keine von denen erstattet werden. In manchen Fällen, wo extra Leistungen mit besonderer Bedienung (sogenannte Privat-Abteilungen) angeboten werden, wird ein Anteil der Betriebskosten von Patient erstattet.

\section{GRUNDLAGE DER KOSTEN- UND LEISTUNGSRECHNUNG IM KRANKENHAUS}

Der Kosten- und Leistungsrechnung kommt im Rahmen einer angewandten Betriebsführung allgemein die Aufgabe zu, kostenorientierte betriebliche Entscheidungsprozesse zu erklären sowie den für die Zielrealisierung erforderlichen Einsatz von Wirtschaftsgütern festzulegen. ${ }^{11}$ Diese Aussage gilt auch für die Kosten- und Leistungsrechnung im Krankenhaus mit ihren speziellen, durch die besondere Zielsetzung dieser Dienstleistungsbetriebe bedingten Problemen. In diesem Kapitel werden die Grundlagen der Kosten- und Leistungsrechnung im Allgemeinen und im Hinblick auf Krankenhäuser behandelt.

\subsection{Allgemeine Betrachtungen zur Kosten- und Leistungsrechnung in Krankenhaus}

Grundsätzlich kann es unterstellt werden, dass die mengen- und wertmäßige Erfassung, Verteilung und Zurechnung von Kosten in einem Krankenhaus der gleichen Problematik unterliegt wie in einem erwerbswirtschaftlichen Unternehmen. Der allgemeine Gemeinsamkeit bezüglich der grundsätzlich vom Unternehmenstyp unabhängigen Tragfähigkeit der konzeptionellen Grundlagen der Kosten- und Leistungsrechnung befreit immerhin nicht von einer jeweils unternehmenstypspezifischen Auslegung der Grundbegriffe und einer betriebszielorientierten Gestaltung einer Kosten- und Leistungsrechnung.

\footnotetext{
111 Vgl. Freidank, C. (1994): Kostenrechnung, Einführung in die begrifflichen, theoretischen, verrechnungstechnischen sowie planungs- und kontrollorientierten Grundlagen des innerbetrieblichen Rechnungswesens, 5. Aufl., München, Wien: Oldenburg, 1994, S. 1.
} 


\subsubsection{Aufgaben der Kosten- und Leistungsrechnung}

Eines der wichtigsten Hilfsmittel, durch die eine ordnungsgemäße Planung, Steuerung, Überwachung und Kontrolle eines Kombinationsprozesses gewährleistet wird, ist das betriebliche Rechnungswesen. Das betriebliche Rechnungswesen umfasst sämtliche Verfahren, die dazu dienen, das betriebliche Geschehen zahlenmäßig zu erfassen, zu planen und zu kontrollieren. ${ }^{112}$

Die Hauptaufgaben des Rechnungswesens, die sowohl außenstehende (externe) als auch interne Interessenten dienen, lassen sich durch die Zweckerfüllung des Rechnungswesens ableiten. ${ }^{113}$ Sie haben abstrakt - formalen Inhalt und charakterisieren in allgemeiner Form, die Art und Verwendung der bereitzustellenden Informationen. Die Hauptaufgaben sind die: ${ }^{114}$

- Dokumentationsaufgabe: Sachlich und zeitlich geordnete Aufzeichnung aller Geschäftsfällen.

- Rechenschaftslegungs- und Informationsaufgabe: Rechenschaftslegung und Information über die Lage der Vermögen, der Schulden und des Erfolges des Unternehmens.

- Dispositionsaufgabe: Bereitstellung des aufbereiteten Zahlenmaterials für Planungs- und Entscheidungszwecke.

- Kontrollaufgabe: eingerichteten Informations- und Kontrollsystem für Überwachung der Wirtschaftlichkeit sowie Zahlungsfähigkeit des Unternehmens.

Die Erfüllung der Zwecke des betrieblichen Rechnungswesens hängt von den vorgegebenen, zu erreichenden Zielen ab (Liquiditäts- und Erfolgsziel). ${ }^{115}$ Aus der Verschiedenheit der Aufgaben der betrieblichen Rechnungswesens ergibt sich eine

${ }^{112}$ Vgl. Haberstock, L.: Kostenrechnung,. Einführung mit Fragen, Aufgaben und Lösungen, Bd.1, 8. Aufl., Wiesbaden, 1987, S.7.

113 Wedel, H. (1999): Grundlagen des Rechnungswesens, Band 2: Kosten- und Leistungsrechnung, 7. Aufl., Herne, Berlin, 1999, S.14.

${ }^{114}$ Vgl. Schoenfeld, H., Möller, H. (1995): Kostenrechnung, Einführung in das betriebswirtschaftliche Rechnungswesen mit Erlöse und Kosten, 8. Aufl., Stuttgart, 1995, S.4-10.

115 Vgl. Bloech, J., Götze, U., Sierke, B. (1993): Managementorientiertes Rechnungswesen, Konzepte und Analysen zur Entscheidungsvorbereitung, Wiesbaden, 1993, S. 10. 
Zweiteilung in externes Rechnungswesen (Finanzbuchführung und Jahresabschluss) und interne Rechnungswesen ( Kosten- und Leistungsrechnung) ${ }^{116}$

Die Aufgabenschwerpunkte und Informationsempfänger des betrieblichen Rechnungswesens (externes und internes) sind in Abb. 12 dargestellt.

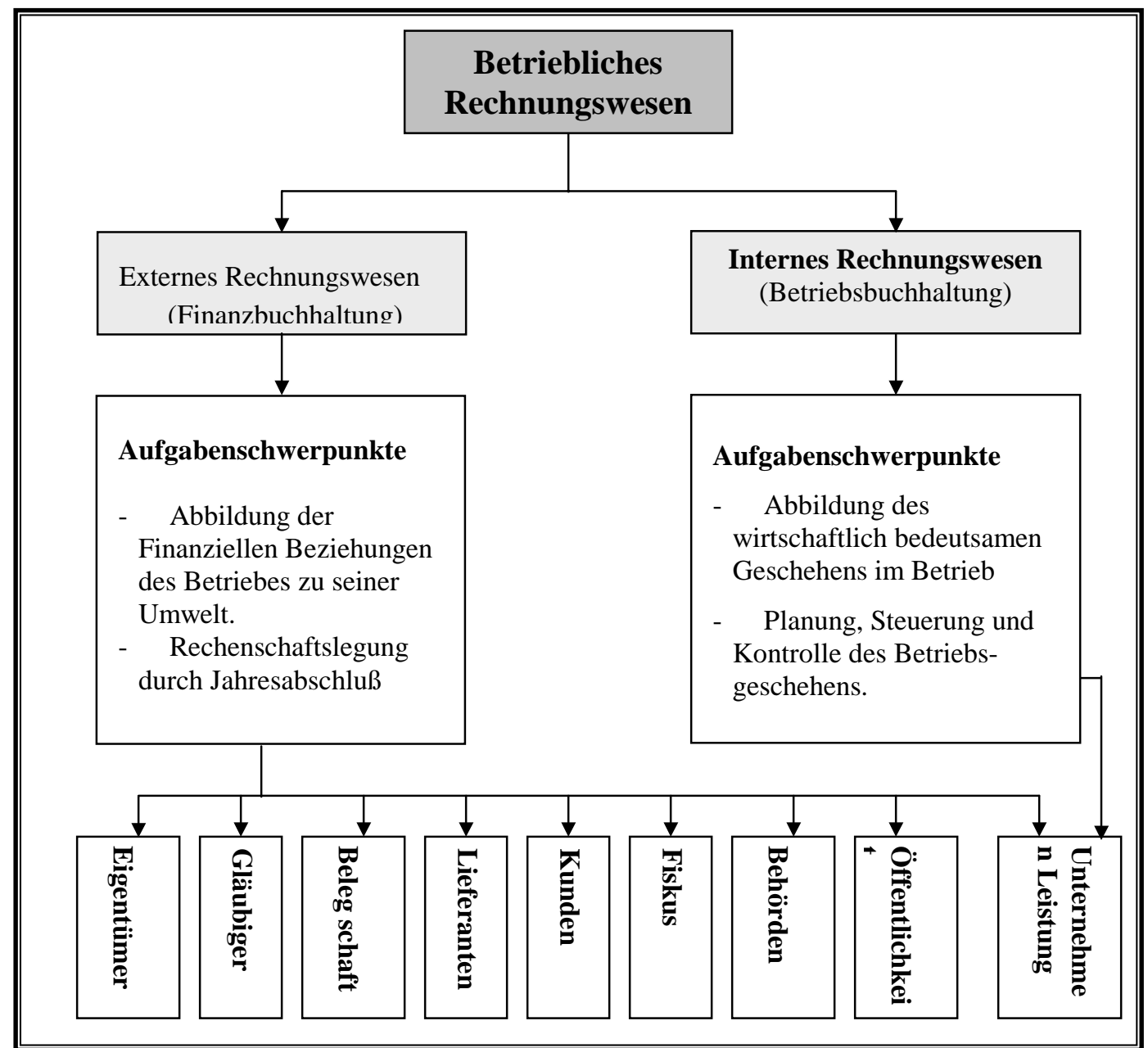

Abb. 12: Aufgabenschwerpunkte und Informationsempfänger des betrieblichen Rechnungswesens. ${ }^{117}$

Die Kosten- und Leistungsrechnung stellt einen Teilbereich des betrieblichen Rechnungswesens dar, der durch eine systematische Erfassung, Weiterleitung und

116 Vgl. Fuchs, M. (1988): Grundlagen des betrieblichen Rechnungswesens, in: Eichhorn, S. (Hrsg.), Handbuch Krankenhaus-Rechnungswesen, 2. Aufl., Wiesbaden, 1988, S. 41-42.

117 Quelle: Hummel, S., Männel, W. (1986): Kostenrechnung, Grundlagen, Aufbau und Anwendung, 4. Aufl., Wiesbaden, 1986 S.6. 
Aufbereitung von Kosten und Leistungen der Unternehmensführung Informationen für eine betriebsinterne Planung, Steuerung und Kontrolle bereitstellen soll. ${ }^{118}$

Gegenstand der Finanzbuchführung (Geschäftsbuchführung) sind alle monetären Vorgänge zwischen dem Krankenhaus und der Umwelt. Die Kosten- und Leistungsrechnung erfasst hingegen den Prozess der Leistungserstellung und Leistungswertung innerhalb des Krankenhauses. ${ }^{119}$ Die Zielsetzung der Wirtschaftlichkeit der krankenhausbetrieblichen Leistungserstellung ist die Kostenminimierung für ein vorgegebenes Leistungsprogramm. Dies verlangt ein Instrumentarium, das zur Überprüfung des Zielerreichungsgrades geeignet ist. Ein mittelbar notwendiges Instrument dafür ist die Kosten- und Leistungsrechnung. ${ }^{120}$

Während bei der nach außen gerichteten Finanzbuchführung mit dem Jahresabschluss die Rechenschaftslegungs- und Informationsaufgabe im Vordergrund steht, bildet das interne Rechnungswesen den Betriebsprozess in Kosten- und Leistungsgrößen ab. Damit werden die zwei Hauptzwecke erfüllt: ${ }^{121}$

- Kosten- und leistungsbezogene Planung, Steuerung und Kontrolle des Betriebsprozesses sowie

- Preisermittlung.

Eine dritte Aufgabe könnte allerdings als Aspekt der Kosten- und Leistungsrechnung für dispositive Zwecke, nämlich das Bereitstellen des Zahlenmaterials, benannt werden, ${ }^{122}$ wenn sie den instrumental programmatischen Charakter im Zusammenhang mit unternehmerischen Entscheidungs- und Steuerungsaufgaben betonen. $^{123}$

Für das Krankenhaus muss die Kosten- und Leistungsrechnung im Rahmen der Planung, Steuerung und Kontrolle des Betriebsprozesses insbesondere Informationen über die Wirtschaftlichkeit zur Verfügen stellen. ${ }^{124}$ An die Stelle der Preis-

\footnotetext{
118 Vgl. Hummel, S., Männel, W.(1986) S. 4.

119 Vgl. Hentze, J., Kehres, E. (1995): Kosten- und Leistungsrechnung in Krankenhaus, systematische Einführung, 3. Aufl., Stuttgart, Berlin, Köln: Kohlhammer, 1995, S. 20.

${ }^{120}$ Vgl. Leonhardt, J. (1988): Aufbau- und Anwendungsmöglichkeiten einer Grenzplankostenrechnung in Krankenhausbetrieben, Hamburg, 1988, S. 14.

${ }^{121}$ Vgl. Schweitzer, M., Küpper, H.: Systeme der Kostenrechnung, 5. Aufl., Landsberg, 1991, S. 58.

122 Vgl. Kosiol, E. (1979 A): Kosten- und Leistungsrechnung, Berlin,1979, S.85.

123 Vgl. Schweitzer, M., Küpper, H. (1991), S. 18.

${ }^{124}$ Vgl. Hentze, J., Kehres, E. (1995), S. 20.
} 
ermittlung tritt die Ermittlung der Selbstkosten, die gleichzeitig auf gültige Gesundheitsgrundrechte und Entgeltsformen reagieren müsste. ${ }^{125}$

Eine Hilfsfunktion der Kosten- und Leistungsrechnung wird besonders deutlich, wenn es im Bereich der Planung und Kontrolle um die Leistung geht. Hier ist aber die Kosten- und Leistungsrechnung sicher nicht der einzige Datenlieferant.

Der Aufgabenkomplex der Kosten- und Leistungsrechnung im Krankenhaus findet sich grundsätzlich auch in privaten Betrieben. Im Einzelnen jedoch ergeben sich zusätzliche Aufgaben durch die staatliche Kontrolle der Kosten und Leistung. Das soll die folgende Auffächerung der Aufgaben verdeutlichen (Tab. 1).

\begin{tabular}{|c|c|c|}
\hline \multirow{2}{*}{$\begin{array}{l}\text { Aufgabenkom- } \\
\text { plexe }\end{array}$} & \multicolumn{2}{|l|}{ Einzelaufgaben } \\
\hline & $\begin{array}{l}\text { Innerhalb des } \\
\text { Krankenhauses }\end{array}$ & nach außen \\
\hline Dokumentation & 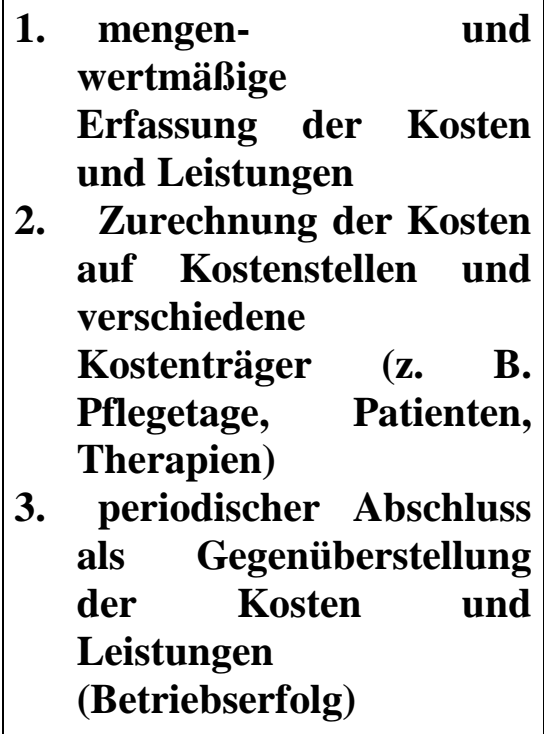 & $\begin{array}{l}\text { 1. } \begin{array}{l}\text { und } \\
\text { wengen- }\end{array} \\
\text { der Kortmäßige Erfassung } \\
\text { Leistungen } \\
\text { 2. Zurechnung der Kosten } \\
\text { auf Kostenstellen } \\
\text { 3. Nachweis der Selbst- } \\
\text { Kosten. }\end{array}$ \\
\hline Planung & 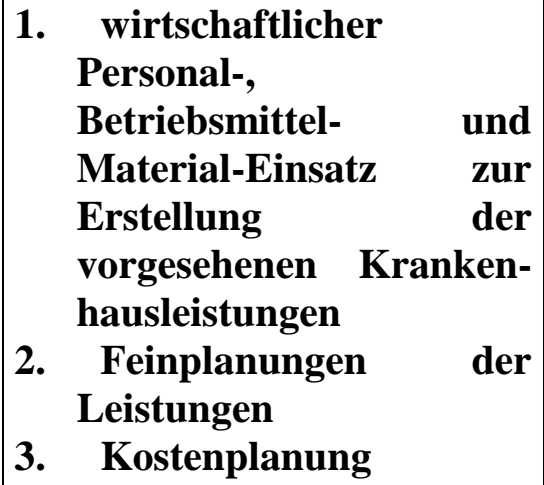 & $\begin{array}{l}\text { regionale, quantitative } \\
\text { und qualitative } \\
\text { Krankenhaus-Planung }\end{array}$ \\
\hline
\end{tabular}

125 Vgl. Dieter, B. (1994): Understanding the Hospital Cost Accounting Process, in: Finkler, S. (Edit.), Issues in Cost Accounting for Health Care Organisations, New York, 1994, S. 13. 


\begin{tabular}{|c|c|c|}
\hline Kontrolle & $\begin{array}{l}\text { 1. Beobachtung von Kosten } \\
\text { und Leistungen im } \\
\text { Zeitablauf } \\
\text { 2. Ermittlung } \\
\text { Kennzahlen } \\
\text { 3. Soll/Ist-Vergleich }\end{array}$ & $\begin{array}{l}\text { Zur Beurteilung der } \\
\text { sparsamen } \\
\text { Wirtschaftsführung: } \\
\text { 1. Zwischen- und über- } \\
\text { betriebliche Vergleiche } \\
\text { der Kosten und } \\
\text { Leistungen } \\
\text { 2. Schaffung von Kosten- } \\
\text { und Leistungsstandards }\end{array}$ \\
\hline
\end{tabular}

Tab. 1: Aufgaben der Kosten- und Leistungsrechnung im Krankenhaus. ${ }^{126}$

\subsubsection{Begriffsdefinitionen zur krankenhausspezifischen Kosten- und Leistungs- rechnung}

In den verschiedenen Teilgebieten des betrieblichen Rechnungswesens wird mit unterschiedlichen ökonomischen Größen gerechnet, die teils übereinstimmende und teils abweichende Inhalte haben und für die sich spezifische Begriffe bezüglich eines Krankenhauses herausgebildet haben.

Kosten und Leistungen sind ein gleichrangiges Begriffspaar. Kosten entstehen durch Leistungen, während die Leistungserstellung Kosten verursacht. Beide, Kosten und Leistungen, sind sowohl als Mengen als auch als Werte zu verstehen. Die erforderlichen Ressourcen (Produktionsfaktoren) werden in den verschiedenen Teilbereichen des Krankenhauses vorgehalten und zur Leistungserstellung eingesetzt, um das Hauptziel, die stationäre und ambulante Versorgung der Krankenhaus-Patienten, zu erreichen. Die damit verbundenen Kosten können, müssen aber nicht identisch sein mit der Gesamtsumme aller Krankenhaus-Rechnungen. Abb. 13 stellt Kosten und Leistung als einander bedingende Größen dar.

\footnotetext{
126 In Anlehnung an: Vgl. Bopp, M., Hohenbild, R. (1984): Kosten- und Leistungsrechnung im Krankenhaus, Köln, 1984, S. 11.
} 


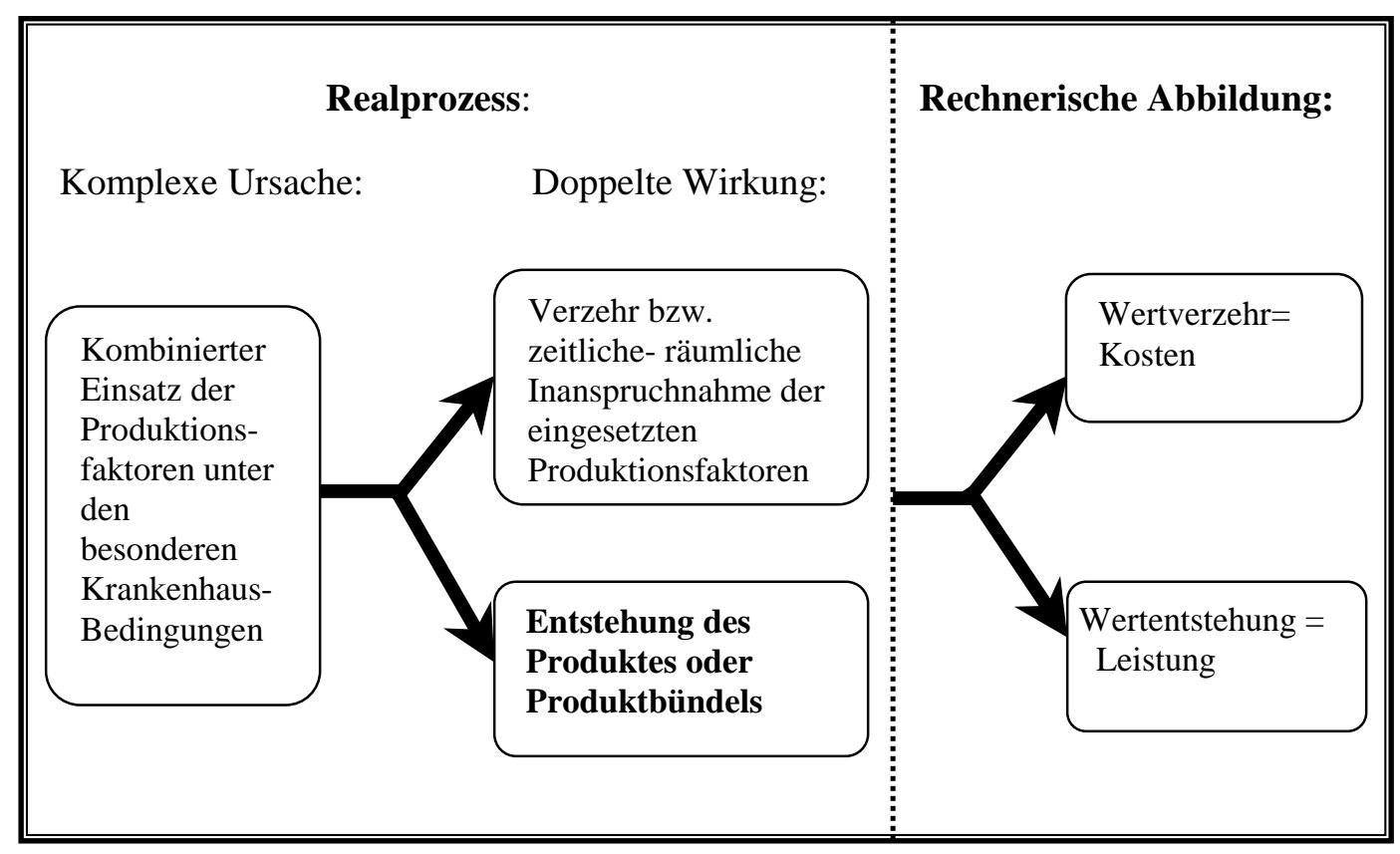

Abb. 13: Kosten und Leistung als einander bedingende Größen ${ }^{127}$

\subsubsection{Kostenbegriff}

Die Festlegung des Kostenbegriffs kann in einer wertmäßigen oder in einer pagatorischen Sichtweise erfolgen. ${ }^{128}$

Der wertmäßige Kostenbegriff bestimmt Kosten als bewerteten, leistungsbezogenen Verzehr von Gütern und Dienstleistungen einer Rechnungsperiode. ${ }^{129}$ Unabhängig von der Auszahlungs- bzw. Ausgabenwirksamkeit können bei Anwendung des wertmäßigen Kostenbegriffs kalkulatorische Kosten in Form von Anders- oder Zusatzkosten in Ansatz gebracht werden. ${ }^{130}$

Die Mehrzahl der Autoren verwendet den wertmäßigen Kostenbegriff, der auch in der Praxis des Rechnungswesens die weiteste Verbreitung gefunden hat. Kosten sind danach bewerteter Verzehr von wirtschaftlichen Gütern und Dienstleistungen,

127 Quelle: Hildbrand, R. (1988): Kostenrechnung, in: Eichhorn, S. (Hrsg.), Handbuch Krankenhausrechnungswesen - Grundlagen- Verfahren- Anwendungen, 2. Aufl., Wiesbaden, 1988, S. 373. ${ }^{128}$ Vgl. Eisel, W. (1994): Technik des betrieblichen Rechnungswesens, München, 1980, S. 339.

${ }^{129}$ Vgl. Freidank, C., (1994), S. 4.

${ }^{130}$ Zusatzkosten sind Kosten, die nur kalkulatorisch in die Kostenrechnung eingehen, also keinen Aufwand darstellen, (z.B. kalkulatorischer Unternehmerlohn, kalkulatorische Eigenkapitalzinsen, oder kalkulatorische Eigenmiete). Anderskosten sind Kosten, die in der Kostenrechnung nach anderen Gesichtspunkten als in der Aufwandsrechnung berücksichtigt werden, (z. B. kalkulatorische Abschreibungen, kalkulatorische Zinsen, oder kalkulatorische Wagnisse. 
der zur Erreichung des Betriebszweckes sowie zur Aufrechterhaltung der erforderlichen Kapazitäten entsteht.

Unabhängig von verschiedenartigen Formulierungen ist dieser Begriff durch drei Merkmale gekennzeichnet: ${ }^{131}$

- Verbrauch von Wirtschaftsgütern: Wirtschaftsgüter sind Gegenstände, die real existieren, zweckgeeignet, rechtlich verfügbar, übertragbar und knapp sind. Zum Güterverbrauch zählt nicht nur der Verbrauch an Sachgüter, sondern auch die Nutzung von Arbeitsleistungen, Dienstleistungen, Nutzungsrechten und Kapital.

- Leistungsbezogenheit des Güterverbrauchs: Der Güterverbrauch wird eingeschränkt auf denjenigen Teil, der bewußt eingegangen wird bzw. auf Grund naturgesetzlicher, rechtlicher und wirtschaftlichen Daten nicht vermeidbar ist, wenn man Wirtschaftsgüter zum Zweck der Leistungserstellung einsetzt.

- Bewertung der Leistungsbezogenheit des Güterverbrauchs: Durch die Bewertung mit Geldeinheiten werden die ungleichnamigen, leistungsbezogenen Kostengüterverbrauchsmengen gleichnamig gemacht und damit verrechenbar. Wie die Bewertung zu erfolgen hat (z. B. Anschaffungspreise, Wiederbeschaffungspreise, Festpreise, Opportunitätskosten), richtet sich nach dem Zweck der Rechnung.

Der pagatorische Kostenbegriff leitet sich im Gegensatz zum wertmäßigen Kostenbegriff nicht aus Güterbewegungen, sondern aus Geldbewegungen ab. ${ }^{132}$ Kosten werden als betriebsbezogene, nicht kompensierte Ausgaben definiert. Dieser Begriff ist abzugrenzen von Begriffen der pagatorischen Rechnung, die auf die Erfassung von Zahlungsströmen abstellt. Hierbei leistungsbezogenen Verbrauch wird mit den zugehörigen historischen oder planmäßigen Anschaffungspreisen (ausgaben) bewertet. ${ }^{133}$

${ }^{131}$ Vgl. Bopp, M., Hohenbild, R. (1984): Kosten- und Leistungsrechnung im Krankenhaus, Köln 1984. S.16-21 und vgl. Freidank, C., (1994), S. 4-9.

${ }^{132}$ Vgl. Heinen, E. (1978): Betriebswirtschaftliche Kostenlehre, 5. Aufl., Wiesbaden, 1978, S. 81.

${ }^{133}$ Vgl. Freidank, C., (1994), S. 9-10. 


\subsubsection{Leistungsbegriff}

In der Kosten- und Leistungsrechnung wird Leistung nicht als physikalische Größe oder als Tätigkeit aufgefasst, sondern als Ergebnis betrieblicher Tätigkeit. Dieses Tätigkeitsergebnis hat, wie die Kosten, eine Mengen- und eine Wertkomponente, die multiplikativ zu einer Wertgröße verbunden werden.

Als Korrelat zum wertmäßigen Kostenbegriff sind Leistungen im Produktionsprozess entstandene, bewertete Wirtschaftsgüter. Dieser Begriff lässt sich prinzipiell durch drei Merkmale kennzeichnet. ${ }^{134}$

- Entstehung von Wirtschaftsgütern: Der Begriff der Wirtschaftsgüter schließt Sachgüter, Dienstleistungen und Rechte ein. Allgemein umfassen Leistungen alle Tätigkeitsergebnisse, denen Kosten zugerechnet werden können. Dabei entstehen aus den eingesetzten Güter- und Dienstleistungen durch Kombination und Transformation neue Güter und Dienstleistungen, die entweder nach außen oder innerbetrieblich weiter verwertet werden.

- Bezogenheit auf den Produktionsprozess: Diese Bezogenheit drückt aus, dass zu den Leistungen nur diejenigen Güter- und Dienstleistungen zählen, die der eigentlichen (typischen) betrieblichen Aufgabenerfüllung dienen.

- Bewertung der hergestellten Produkte: Die heterogenen Güter- und Dienstleistungen werden durch die Bewertung vergleichbar und verrechenbar gemacht. Die Verrechenbarkeit ist die Voraussetzung für die Erfassung und Auswertung der Leistungshöhe. In der Leistungsrechnung werden allgemein folgende Wertansätze verwendet: Verkaufs-, Fest-, Durchschnitts-, Schätz- und Knappheitspreise. Die Verkaufspreise sind das wertmäßige Äquivalent der eigentlichen betrieblichen Tätigkeit, die im Krankenhaus vornehmlich aus stationären und ambulanten Leistungen bestehen. Im Krankenhaus als Dienstleistungsbetrieb ist das Endprodukt der Leistung als „Gesundheit“ nicht messbar, doch sind Messung und Bewertung der Einsatzfaktoren, die zum Endpro-

${ }^{134}$ Vgl. Scherrer, G. (1999): Kostenrechnung, 3., neubearb. Aufl., Stuttgart, 1999, S.13-14. 
dukt führen, genauso Elemente zur Leistungsermittlung wie in der Kostentheorie. $^{135}$

Von den wertmäßigen Leistungen sind die Erträge zu unterscheiden. Während die Leistung den Wert aller im Rahmen der betrieblichen Tätigkeit erstellten Güterund Dienstleistungen erfasst, beinhalten die Erträge erzielten Gegenwerte für die veräußerten mengenmäßigen Leistungen.

Beim pagatorischen Leistungsbegriff erfolgt die Bewertung der hergestellten Produkte mit dem realisierten Beitrag als Einzahlungsströmen aus der Veräußerung der Produkte. ${ }^{136}$

\subsection{Kosten- und Leistungsrechnung in deutschen Krankenhäusern}

In diesem Abschnitt werden die Grundlagen der Kosten- und Leistungsrechnung in deutschen Krankenhäusern betrachtet.

\subsubsection{Aufgaben der Kosten- und Leistungsrechnung in deutschen Kranken- häusern}

Die Kosten- und Leistungsrechnung als Teil des betrieblichen Rechnungswesens ist fast ausschließlich auf interne Zwecke ausgerichtet. Eine Ausnahme besteht allerdings für solche Betriebe, die bestimmte öffentliche Aufträge abwickeln, wobei die im Betrieb angefallenen Kosten gegenüber dem Auftraggeber nachgewiesen werden. ${ }^{137}$ Für das Verfahren der Preisbildung und zum Zwecke der Informationsaufbereitung für öffentlich Auftraggeber ist die Kosten- und Leistungsrechnung an die Vorschriften der „Verordnung über die Preise bei öffentlichen Aufträgen“ und der „Leitsätze für die Preisermittlung auf Grund von Selbstkosten“ gebunden. ${ }^{138}$

Die Kosten- und Leistungsrechnung für deutsche Krankenhäuser ist durch $\S 8$ KHBV gesetzlich vorgeschrieben und muss neben den allgemeinen betriebs-

\footnotetext{
${ }^{135}$ Vgl. Tauch, J. (1987): Kosten- und Leistungsrechnung im Krankenhaus, von Kosten- und Leistungsnachweis zur Patientenbezogenen Kosten- und Leistungsrechnung, 1. Aufl., Gütersloh, 1987.S. 21.

${ }^{136}$ Vgl. Scherrer, G., (1999), S.15.

137 Vgl. Brede, H. (1997): Betriebswirtschaftslehre, Einführung, 6., unwesentl. veränd. Aufl. München, Wien, 1997, S. 209.
} 
wirtschaftlichen Funktionen auch rechtlich vorgegebene Zwecke erfüllen. Dadurch erhält die Kosten- und Leistungsrechnung des Krankenhauses ebenfalls eine Sonderstellung. ${ }^{139}$ Indem sich die Vergütung der deutschen Krankenhäuser für eine längeren Übergangszeit aus einem Mischsystem von

- Kostenerstattungspreisen und

- pauschalierten Festpreisen

zusammensetzt, entstehen daraus gleichzeitig vielschichtige und komplexe Anforderungen an die Aufgaben und die Ausgestaltung der Kosten- und Leistungsrechnung. ${ }^{140}$ Die Aufgaben der Kosten- und Leistungsrechnung in deutschen Krankenhäusern lassen sich an zwei Aspekten festmachen: gesetzliche Dokumentationsgrundlage und die als Instrument der Entscheidungsunterstützung, auf die in den folgenden beiden Abschnitten näher eingegangen wird.

\subsubsection{Die Kosten- und Leistungsrechnung als gesetzliche Dokumentations- grundlage}

Die rechtlich vorgegebenen Zwecke der Kosten- und Leistungsrechnung bestimmen sich vor allem aus $\S 8$ Abs. $1 \mathrm{KHBV}$. Jedes Krankenhaus ist gemäß $\S 8$ Abs. 1 verpflichtet, eine Kosten- und Leistungsrechnung zu führen, die aufbauend auf dem Zahlenwerk der Finanzbuchhaltung folgende Aufgaben erfüllen muss:

- Innerbetriebliche Steuerung,

- Ermittlung und Beurteilung der Wirtschaftlichkeit und Leistungsfähigkeit,

- Ermittlung der pflegesatzfähigen Kosten für das Erstellen der Leistungsund Kalkulationsaufstellung nach den Vorschriften der BPflV.

\section{Betriebsinterne Steuerung:}

138 Vgl. Götzinger, M., Michael, H. (1990): Kosten- und Leistungsrechnung, eine Einführung, 5. Aufl., Heidelberg, 1990, S. 24.

139 Vgl. Freymann, H. (1989): Controlling in Krankenhaus - Möglichkeiten und Nutzen für die Betriebsführung, in: Eichhorn, s., Feyermann, H. (Hrsg.), Gesamtverantwortung, Wirtschaftliches Krankenhaus, Beiträge zu Management, Planung, Rechnungswesen, Prüfung, 3. Aufl., Köln, 1989, S. 61.

140 Vgl. Schlüchtermann, J., Gorschlüter, P. (1996): Ausgewählte Aspekte des modernen Kostenmanagements, In: Adam, D. (Hrsg.), Krankenhausmanagement, Schriftenreihe zur Unternehmensführung, Band 59, Wiesbaden 1996, S. 98. 
Gemäß $\S 8 \mathrm{KHBV}$ hat das Krankenhaus eine Kosten- und Leistungsrechnung, die eine betriebsinterne Steuerung erlaubt, wobei es keine gesetzlichen Vorschriften über die Ausgestaltung der Kosten- und Leistungsrechnung gibt. Sie stellt ab auf das Erreichen von Zielen, die quantifizierbar nach Zielinhalt, Zielausmaß und zeitlichem Bezug vorgegeben sind. ${ }^{141}$ Eine eindeutige Zielsetzung ist hier Voraussetzung für eine effektive Steuerung. Nur wenn das Ziel klar definiert ist, kann die betriebliche Steuerung sinnvoll sein. ${ }^{142}$

Das gesetzlich vorgeschriebene Ziel des deutschen Krankenhauses ist die Erfüllung des Versorgungsauftrages. Das bedeutet, die Verbesserung des Gesundheitsstatus aller das Krankenhaus aufsuchenden Patienten herbeizuführen. Daher wird als Primärleistung der Krankenhausleistung auch die Veränderung des Gesundheitsstatus, d. h. die Gesundheitszustandsveränderung des Patienten definiert. ${ }^{143}$ Die Operationalisierung dieses globalen Zieles bestimmt die qualitative und quantitative Gesamtleistung des Krankenhauses für zukünftige Perioden. ${ }^{144}$ Die Krankenhausgesamtleistung lässt sich aus dem Leistungsspektrum an Fächern, stationärer und ambulanter Patientenversorgung sowie die dafür notwendige Infrastruktur beschreiben. ${ }^{145}$ Für die Planung muss der zukünftige Bedarf an geplanten Einzelleistungen ermittelt werden, um die notwendigen Ressourcen bereitstellen zu können. ${ }^{146}$ Durch die gesetzlich zwingende Festschreibung des Budgets ist es für die Existenzsicherung der Krankenhäuser unvermeidbar, in unterjährigen Periodenabständen die realisierten Leistungen den vereinbarten Leistungen gegenüberzustellen, um bei Abweichungen Gegensteuerungsmaßnahmen einleiten zu können. ${ }^{147}$

\section{Ermittlung und Beurteilung der Wirtschaftlichkeit und Leistungsfähigkeit:}

Die Ermittlung und Beurteilung und der Wirtschaftlichkeit ist insbesondere im Krankenhaus ein wesentlicher Zweck der Kosten- und Leistungsrechnung. ${ }^{148}$ Ent-

\footnotetext{
${ }^{141}$ Vgl. Hentze, J., Kehres, E. (1995), S. 21.

142 Vgl. Hildbrand, R. (1988), S. 348.

${ }^{143}$ Vgl. Hörmann, W., Ingruber, H. (1988), S. 9-28.

144 Vgl. Möhlmann, E. (1992), S. 87-92.

145 Die Sekundärleistungen sind in $\S 2$ Abs. 1 BPflV abschließend aufgezählt.

146 Vgl. Keun, F. (1997), S. 83.

${ }^{147}$ Vgl. Hildebrand, R. (1988), S. 358.

${ }^{148}$ Vgl. Keun, F. (1997), S. 84.
} 
sprechend der Aufgabenstellung der deutschen Krankenhäuser, nämlich Krankheiten festzustellen, zu heilen oder zu lindern, kommt die Leistungsfähigkeit eines Krankenhauses primär darin zum Ausdruck, ${ }^{149}$ inwieweit es gelingt, den Gesundheitszustand von Patienten positiv zu verändern. Gemäß $§ 17$ Abs. 1 KHG müssen die Pflegesätze medizinisch leistungsgerecht sein, und einem Krankenhaus bei wirtschaftlicher Betriebsführung ermöglichen, den Versorgungsauftrag zu erfüllen. ${ }^{150}$

Auch durch den im Gesetz vorgesehenen Krankenhausvergleich werden die Wirtschaftlichkeit und die Möglichkeit der Beurteilung der Wirtschaftlichkeit gewinnen. Die Bundespflegesatzverordnung 1995 hat im §5 einen solchen Krankenhausvergleich ab 1998 vorgesehen. ${ }^{151}$

Die Pflicht zur Beurteilung der Wirtschaftlichkeit und Leistungsfähigkeit eines deutschen Krankenhauses begründet sich darauf, dass die Leistungen zu einem großen Teil auf Basis von Kostenerstattungen finanziert werden müssen. Dabei wird auch beurteilt, ob sich die Kostenforderungen des betreffenden Krankenhauses auf einer wirtschaftlichen Leistungserbringung begründen. ${ }^{152}$

In $§ 13$ BPflV a. F. sind in diesem Zusammenhang die Kosten und Leistungen vergleichbarer Krankenhäuser sowie die Maßstäbe und Grundsätze zur Beurteilung der Wirtschaftlichkeit und Leistungsfähigkeit der Krankenhäuser nach § 19 KHG festgeschrieben. Es ist vorgesehen, dass die deutsche Krankenhausgesellschaft und die Spitzenverbände der gesetzlichen Krankenversicherung Empfehlungen über Maßstäbe und Grundsätze für die Wirtschaftlichkeit der Krankenhäuser, insbesondere für den Personalbedarf und die Sachkosten, erarbeiten. ${ }^{153}$ Die Ermittlung der Kosten- und Leistungsstruktur in den deutschen Krankenhäusern ist nicht nur aus Gründen der betrieblichen Steuerung notwendig. Insbesondere soll eine Transparenz der Kosten- und Leistungsverhältnisse jedes Krankenhauses geschaffen

\footnotetext{
${ }^{149}$ Vgl. Eichhorn, S. (1976), S. 29.

${ }^{150}$ Vgl. 117 Abs.1 KHG.

${ }^{151}$ Vgl. Baschke, W. L. (1994): BPflV 95- der Kompromiß, in: F\&W, 4/1994, S. 270.

152 Vgl. Hentze, J., Kehres, E. (1995), S. 23.

${ }^{153}$ Vgl. §19 KHG.
} 
werden, die den Patienten die Wahl des Krankenhauses und den Krankenkassen einen Vergleich erleichtern. ${ }^{154}$

Gemäß Artikel 10 der Verordnung zur Neuordnung des Pflegesatzrechtes von 08.01.1994 i.V.m. § 5 Abs. 1 BPflV wird durch den vorgesehenen Krankenhausvergleich auf Landesebene die Bemessung von medizinischen leistungsgerechten Budgets sowie von tagesgleichen Pflegesätze mit der krankenhausindividuellen Ermittlung abgestimmt. Beispielsweise wird ein Krankenhausvergleich notwendig sein, wenn die Bemessung des Entgeltes für Fallpauschalen und Sonderentgelte nach durchschnittlichem Punktwert nicht endgültig festgelegt ist und damit einem ständigen Anpassungsprozess unterliegt. ${ }^{155}$ Durch einen Krankenhausvergleich wird die Optimierung der Kosten und der daraus entwickelten Preise möglich sein. Damit müssen Krankenhäuser, deren Kosten über den Preisen liegen, Reserven ausschöpfen, um mindestens kostendeckend zu arbeiten. Krankenhäuser, die eine Kostendeckung erreichen sogar oder Gewinne erzielen, könnten nach weiteren Möglichkeiten der Kosteneinsparung suchen. ${ }^{156}$ Aus diesem Anpassungsprozeß könnten optimierte Durchschnittspreise im Zeitablauf erreichen. ${ }^{157}$

Mit der Änderungen der Gesetze für Krankenhaus wird die Kosten- und Leistungsrechnung im Krankenhaus mehr und mehr ihren Zwecken gerecht, die erforderlichen Informationen zur Beurteilung und Steuerung der Wirtschaftlichkeit in der stationären Krankenversorgung, im einzelnen Krankenhaus, in Abteilungen, Kliniken und Stationen und schließlich der einzelnen erbrachten Leistungen zu liefern. $^{158}$

\section{Ermittlung der pflegesatzfähigen Kosten sowie Erstellung der Leistungs- und Kalkulationsaufstellung (LKA) nach den Vorschriften der BPflV:}

154 Vgl. Monka, M. (1993): Entwicklung und Berechnung von Fallpauschalen, Das ökonomische Modell des WidO, in: Arnold, M., Paffrath, D., Krankenhaus-Report 1993, Aktuelle Beiträge, Trends, Statistik, Stuttgart, 1993, S.98.

${ }^{155}$ Vgl. Keun, F. (1997), S. 86.

156 Vgl. Brayer, F. (1991): Die Kalkulation von Preisen im Krankenhauswesen, Ziele, Methoden und Auswirkungen, Heidelberg, 1991, S. 40.

${ }^{157}$ Vgl. Regler, K. (1994): Auswirkung des GSG und Handlungsbedarf der Krankenhausträger, in: Das Krankenhaus, 1/1994, S. 4.

158 Vgl. Klockhaus, H. E. (1997): Kosten- und Leistungsrechnung im Krankenhaus, München, Essen, 1997, S. 15 
Im $\S 7$ BPflV in Verbindung mit $\S 2$ BPflV wird geregelt, welche Kosten des Krankenhauses zu den Kosten der allgemeinen Krankenhausleistung gehören und durch Budget und Pflegesätze gedeckt werden, und welche Kosten auf andere Weise zu decken sind.

Die stärkere Betonung der Leistungsstruktur eines Krankenhauses im Vorfeld der Pflegesatzverhandlung ist von Bedeutung für die Gestaltung einer krankenhausspezifischen Kosten- und Leistungsrechnung. Nach § 7 Abs. 2 BPflV sind nach dem eingeführten Nettoprinzip in der LKA grundsätzlich nur die pflegesatzfähigen Kosten, d. h. Kosten der allgemeinen voll- und teilstationären Krankenhausleistungen, in der kostenartenspezifischen Gliederung auszuweisen. Die Kosten nicht-pflegesatzfähiger Leistungen, etwa in den Bereichen Ambulanz, krankenhausferne Versorgungseinrichtungen, Personalunterbringung, Forschung und Lehre oder Leistungen an Dritte (wie etwa Arzneimittellieferungen durch die krankenhauseigene Apotheke an andern Krankenhäuser), werden in der LKA nicht mehr ausgewiesen, sondern sind bereits im Vorfeld der Pflegeverhandlungen aus dem Block der gesamten Kosten des Krankenhauses auszugliedern. In Aufweichung des strengen Nettoprinzips sind die nicht-pflegesatzfähigen Kosten anderer Leistungen, die mit der voll- und teilstationären Leistungserbringung in einem engen Bezug stehen, explizit und offen im Rahmen der LKA auszugliedern, so etwa die Kosten vor- und nachstationärer Leistungen, wahlärztlicher Leistungen und letztlich auch die Kosten der durch Fallpauschalen und Sonderentgelte vergüteten Leistungen. Für Vor- und nachstationäre Leistungen gibt es vorerst einen Erlösabzug vom Restbudget (Abteilungsbudget) in Höhe vom 90\% anstelle einer Kostenausgliederung, ${ }^{159}$ d. h. es gibt eine kostenrechnerische Trennung von Pauschalentgelt- und Budgetbereich in Form einer einmaligen Kostenausgliederung, um Quersubventionierungen und daraus resultierende Wettbewerbsvorteile zu unterbinden. ${ }^{160}$

\footnotetext{
159 Erlösabzug bedeutet, dass die pauschalierten Entgelte mit der geplanten Menge der Leistungen multipliziert werden und das Produkt aus dem Abteilungsbudget ausgegliedert wird.

${ }^{160}$ Vgl. Schlüchtermann, J., Gorschlüter, P. (1996), S. 98.
} 


\subsubsection{Die Kosten- und Leistungsrechnung als Instrument der Entschei- dungsunterstützung}

Die Kosten- und Leistungsrechnung in deutschen Krankenhäusern tendierte vor Einführung des Vergütungssystems vor allem dazu, den Aufforderungen aus den KHBV und BPflV gerecht zu werden und nicht dem Krankenhausmanagement ein Informationsinstrument an die Hand zu geben ${ }^{161}$, mit dem eine betriebswirtschaftliche Steuerung und Entscheidungsfindung ermöglicht wird. ${ }^{162}$ Der Grund liegt darin, dass die externen Rechnungslegungsvorschriften in den gesetzlichen Rahmenbedingungen von hoher Bedeutung sind.

An dieser Stelle soll jedoch betrachtet werden, inwieweit die gesetzlichen Mindestanforderungen an die Aufgaben und die Ausgestaltung der krankenhausspezifischen Kosten- und Leistungsrechnung diesen Anforderungen Rechnung tragen bzw. ob aus Sicht der Entscheidungsunterstützung zusätzliche Anforderungen an die Ausgestaltung der Kosten- und Leistungsrechnung zu stellen sind.

Einerseits zeigen die Erfahrungen in der Krankenhauspraxis, dass ausgehend von den externen Rechnungsvorschriften das interne Rechnungsverfahren unmittelbar entwickelt werden muss. Die Instrumente der Kostenermittlung und Kostenkontrolle müssten entwickeln worden, weil es nur mit Kenntnis der Selbstkosten möglich ist, die Wirtschaftlichkeit erbrachter Leistungen nach rechtlichen Rechnungsvorschriften zu beurteilen. ${ }^{163}$

Andererseits entsteht für Krankenhäuser mit der Einführung von wettbewerbspolitischen Anreizmechanismen in Form von Festpreisen ein konkreter Anreiz, die Kosten pro Behandlungsfall so gering wie möglich $\mathrm{zu}$ halten, indem Einsparungspotentiale identifiziert werden und durch Leistungsausweitungen Degressionseffekte einsetzen können. ${ }^{164}$ Für genau definierte Leistungen werden parallel zu den gesetzlichen Anforderungen an die Kosten- und Leistungsrechnung zukünftig entscheidungsorientierte Führungs- und Steuerungsinformation zu stellen sein, mit

\footnotetext{
161 Vgl. Ehrlich, A.(1996): Neuorientierung des Krankenhauscontrolling, in: BiBu, 2/1996, S. 33.

162 Vgl. Klockhaus, H. E. (1997), S. 10.

163 Vgl. Hummel, S., Männel, W.(1986) S. 194.

164 Vgl. Menze, T., Michels, R.: Controlling - Instrumente im Krankenhaus, Existenzsicherung durch mehr Wirtschaftlichkeit, in: F\&W, 3/1996, S. 200.
} 
denen eine langfristige Existenz des Krankenhauses durch das Erschließen von Wettbewerbsvorteilen gesichert werden soll.

Somit ist es nicht mehr sinnvoll, ein Bett möglichst lange mit einem Patienten zu belegen, da dem Krankenhaus unabhängig von der Verweildauer ein konstanter Erlös gegenübersteht. Kurze Verweildauer und viele Fälle pro Bett verbessern die Erfolgslage, indem die durchschnittlichen Behandlungskosten pro Fall sinken. ${ }^{165}$

Mit Einführung der neuen Kosten- und Leistungsrechnung im Rahmen der KHBV und ihrer Änderungensverordnung wird nun die Kalkulation der Entgelte verlangt. Das hat zur Folge, dass auf Entgelte-Ebene eine Beurteilung der Rentabilität per Gesetz verlangt wird. Für die meisten Krankenhäuser wird das bedeuten, dass sie hiermit Neuland beschreiten und sie sich mit der fallbezogenen (gleichzusetzen mit produktbezogenen) Kosten- und Leistungsrechnung erstmals beschäftigen müssen. ${ }^{166}$ Gemäß $§ 8$ Abs. 2 KHBV ist die Steuerung des Unternehmensprozesses durch eine Planung und Kontrolle von Kosten und Leistungen vorgeschrieben. ${ }^{167}$ Unter Steuerung wird hier jedoch lediglich der Einsatz einer Plankostenrechnung verstanden, die das prospektiv zu verhandelnde Budget ermitteln und die Einhaltung geplanter Erlöse und Kosten sichern soll.

Da lediglich durch eine differenzierte Erfassung und Dokumentation der Leistungen die Produktionsfaktoren festgestellt und geplant werden können, muss für die Planung der zukünftige Bedarf an Krankenhausleistungen ermittelt werden, um die notwendigen Ressourcen bereitzustellen. Darüber hinaus setzt die gesetzlich vorgeschriebene betriebsinterne Steuerung eine Kontrolle der Plan-Leistung und Plan-Kosten voraus. ${ }^{168}$

\footnotetext{
165 Bei einem derart auf Rationalisierung ausgelegten System sind zusätzlich gezielte Maßnahmen zur Qualitätssicherung unerläßlich. Es muss verhindert werden, dass der Abbau von Kosten mit einem Abbau von Leistungsqualität einhergeht. Das GSG 93 hat deshalb zusätzlich ein Instrument zur Qualitätssicherung als notwendige Ergänzung für den Rationalisierungsdruck eingeführt, um Patientenselektionen zu unterbinden und den erwünschten Behandlungserfolg sicherzustellen. Vgl. Adam, D. (1996): Krankenhausmanagement im Wandel. In: Adam, D. (Hrsg.): Krankenhausmanagement. Schriftenreihe zur Unternehmensführung (SzU), Band 59, Wiesbaden, 1996, S. 11, Sehen auch 2.2.2.

${ }^{166}$ Vgl. Klockhaus, H. E. (1997), S. 14.

167 Vgl. $\$ 8$ Abs.2 KHBV.

${ }^{168}$ Vgl. Keun, F. (1997), S. 83.
} 


\subsubsection{Rechtliche Rahmenbedingungen der Kosten- und Leistungsrechnung in deutschen Krankenhäusern}

Die Krankenhäuser haben - historisch bedingt - sehr unterschiedliche Motivationen, äußere Formen und innere Organisationen und betätigen sich auf einem Markt, der auf der Angebots- und auf der Nachfrageseite eine ganz besondere Struktur aufweist. Die Besonderheiten der Krankenhäuser haben den Staat veranlasst, alle mit dem Angebot von Krankenhausleistungen zusammenhängenden Fragen gesetzlich zu regeln. ${ }^{169}$ Das gilt im besonderen Maße für das Rechnungswesen.

Zur Erfüllung der Aufgaben des Rechnungswesens werden Informationen über die Situationen des Krankenhausbetriebes allen berechtigten und interessierten Stellen zur Verfügung gestellt. Dazu haben deutsche Krankenhäuser mehr als andere Einzelwirtschaften gesetzliche Vorschriften zu beachten. ${ }^{170}$ Neben allgemeinen Vorschriften, wie handels- und steuerrechtlichen Bestimmungen, zählen hierzu krankenhausspezifische Regelungen wie z. B. das Krankenhausfinanzierungsgesetz (HHG), die Bundespflegesatzverordnung (BPflV), die Krankenhausbuchführungsverordnung (KHBV) sowie weitere landesgesetzliche Regelungen.

\subsubsection{Allgemeine Vorschriften}

$\mathrm{Zu}$ den allgemeinen Vorschriften gehören handelsrechtliche, kommunalrechtliche und steuerrechtliche Vorschriften. ${ }^{171}$

Handelsrechtliche Vorschriften umfassen das Handelsgesetzbuchs (HGB), die Grundsätze ordnungsmäßiger Buchführung $(\mathrm{GoB})$ sowie Bestimmungen über des Rechnungswesens in den für entsprechende Rechtsform von Unternehmen geltende

\footnotetext{
${ }^{169}$ Fuchs, M. (1988), S.50.

${ }^{170}$ Vgl. Keun, F. (1997), S. 6.

${ }^{171}$ Krankenhäuser als Einrichtungen des öffentlichen Gesundheitswesens sind von der Steuerpflicht befreit. Steuerrechtliche Vorschriften, die für andere Unternehmen von großer Bedeutung sind, spielen daher im Bereich des Krankenhauses eine untergeordnete Rolle. Eine Ausnahme bilden die erwerbswirtschaftlichen Privatkliniken.
} 
Gesetzen. ${ }^{172}$ Diese Vorschriften enthalten konkrete Einzelheiten wie Durchführungspflicht, Aufstellung von Bilanz und Bewertung von Vermögen und Schulden.

Nach diesen handelsrechtlichen Vorschriften in Verbindung mit $\S 1$ Abs.1 und $\S 3$ KHBV sind Krankenhäuser verpflichtet, Bücher zu führen und die Vermögenslage nach den Grundsätzen ordnungsmäßiger Buchführung darzulegen.

$\mathrm{Zu}$ den kommunalrechtlichen Vorschriften gehören die Gemeinde- und Kreisordnung, die Bestimmungen über die Haushaltwirtschaft, den Erwerb und die Veräußerung des Vermögens und die wirtschaftliche Betätigung der Gemeinden enthalten, die Eigenbetriebsgesetze und -verordnungen und die für Eigenbetriebe zutreffenden Vorschriften der Gemeindehaushaltsverordnungen und der Gemeindekassenverordnungen der Länder. ${ }^{173}$

\subsubsection{Spezielle Vorschriften}

Das Gesundheitsstrukturgesetz (GSG) von 1993: Das GSG sieht vor allem die Einführung fallorientierter Krankenhausentgelte vor. Darüber hinaus nehmen mit Inkrafttreten des GSG die Aufgaben der Kosten- und Leistungsrechnung im Krankenhaus zu (z. B. Leistungs- und Kosten-Planung, Dokumentation, Kontrolle und Abweichungsanalyse). Durch die gesetzlichen Regelungen des GSG muss die Kostenrechnung die benötigten Daten für den Kosten- und Leistungsnachweis bereitstellen. ${ }^{174}$

Durch das GSG vom 01.01.1993, das als Artikelgesetz aufgebaut ist, wurden die nachstehenden krankenhausspezifischen Vorschriften wesentlich beeinflusst. Diese nachstehend aufgeführten Gesetze bzw. Verordnungen unterliegen bis heute weiteren Änderungen, die als solche nicht mehr auf das GSG zurückzuführen sind, da dieses als Artikelgesetz nur einmalige Wirkung besitzt. ${ }^{175}$

Krankenhausfinanzierungsgesetz (KHG) von 1993

\footnotetext{
172 Krankenhäuser, die in der Rechtsform der AG geführt werden, haben die Vorschriften über die Bildung einer gesetzlichen Rücklage, über die Ergänzung der Gewinn- und Verlustrechnung, über die besonderen Vorschriften zum Anhang und über die Feststellung und Prüfung des Jahresabschlusses zu beachten.

${ }^{173}$ Fuchs, M. (1988), S.56.

${ }^{174}$ Vgl. Strehlau- Schwoll, H. (1993), S. 214.

175 Vgl. Keun, F. (1997), S.8.
} 
Das Gesetz zur wirtschaftlichen Sicherung der Krankenhäuser und zur Regelung der Krankenhauspflegesätze (Krankenhausfinanzierungsgesetze) KHG, das 1972 in Kraft getreten ist, wurde am 10.04.1991 und durch Artikel 11 des GSG 93 modifiziert. ${ }^{176}$ Zuletzt wurde es geändert durch das zweite Gesetz zur Neuordnung von Selbstverwaltung und Eigen Verantwortung in der gesetzlichen Krankenversicherung (2. GKV-NOG) vom 23. 06. 1997. ${ }^{177}$

Zweck dieses Gesetzes ist die wirtschaftliche Sicherung der Krankenhäuser, um eine bedarfsgerechte Versorgung der Bevölkerung mit leistungsfähigen, eigenverantwortlich wirtschaftenden Krankenhäusern $\mathrm{zu}$ gewährleisten ${ }^{178}$ und $\mathrm{zu}$ sozial tragbare Pflegesätze zu ermitteln. ${ }^{179}$

Aus den Bestimmungen des KHG ergeben sich vor allem die folgenden Angelegenheiten für die Kostenrechnung:

- Im Anwendungsbereich stellt sich die Frage, welche Krankenhäuser und welche Einrichtungen in Krankenhäusern überhaupt in die Förderung einbegriffen werden. ${ }^{180}$

- Im Rahmen der Grundsätze für die Pflegesatzregelung sind die Kosten der Krankenhausleistungen nach Maßgaben der KHBV auf Grundlage der kaufmännischen Buchführung und einer Kosten- und Leistungsrechnung zu ermitteln. $^{181}$

- Nach § 28 Abs. 1 KHG sind Krankenhäuser verpflichtet, dem Bundesminister für Gesundheit sowie den zuständigen Behörden der Länder bestimmte Informationen z. B. über Pflegesätze -Beurteilung. -Bemessung und -Entwicklung, die personelle und sachliche Ausstattung und die Kosten der Krankenhäuser nach Kostenarten zu erteilen.

176 In der Fassung der Bekanntmachung vom 10. April. 1991, (BGBI. I, S. 886).

177 BGBI. I, S. 1520.

178 Mit Rationalisierungsinvestitionen und einer Produktivitätssteigerung nach $\S 18$ KHG wurde die Chance zu einer Senkung der durchschnittlichen Kosten vergeben. Vgl. Tanski, J. S.: Finanzierung von Rationalisierungsinvestitionen nach § 18b KHG, in: das Krankenhaus, 2/1997, S. 55

${ }^{179} \mathrm{Vgl}$. KHG $§ 1$ Abs. 1.

180 Z. B. findet dieses Gesetz keine Anwendung auf Krankenhäuser, deren Träger der Bund ist, Polizeikrankenhäuser und Krankenhäuser im Straf- oder Maßregelvollzug ( 3 KHG).

181 § 17 Abs. 2 KHG. 


\section{Krankenhaus- Buchführungsverordnung (KHBV)}

Im Rahmen des $\S 16$ KHG hat die Bundesregierung mit Zustimmung des Bundesrates eine Verordnung über die Rechnungs- und Buchführungspflichten von Krankenhäusern erlassen (Krankenhaus-Buchführungsverordnung KHBV). Diese Verordnung ist am 11.04.1978 in Kraft getreten. Ihre Vorschriften waren erstmals für das am 01.01.1979 beginnende Geschäftsjahr anzuwenden.

Die KHBV gilt für alle Krankenhäuser, auch für die nicht nach KHG geförderten Einrichtungen. Ausgenommen sind nur Krankenhäuser, die nach § 1 Abs. 2 KHG bestimmt werden:

- Krankenhäuser, deren Träger der Bund, eine gesetzliche Rentenversicherung oder eine gesetzliche Unfallversicherung ist,

- Polizeikrankenhäuser,

- Krankenhäuser im Straf- oder Maßregelvollzug und

- die nach $§ 5$ Abs. 1 KHG nicht geförderten Krankenhäuser. ${ }^{182}$

Die KHBV stellt die Mindestforderung an das Krankenhausrechnungswesen dar. Sie verpflichtet Krankenhäuser, die Bücher nach den Regeln der doppelten kaufmännischen Buchführung zu führen (Finanzbuchführung und Jahresabschluss). Daneben hat jedes Krankenhaus gemäß $\S 8$ und der Anlage 5 zur KHBV eine Kosten- und Leistungsrechnung zu führen. Die KHBV umfasst die folgenden fünf Anlagen:

- Anlage 1: Gliederung der Bilanz

- Anlage 2: Gliederung der Gewinn- und Verlustrechnung

- Anlage 3: Anlagennachweis

- Anlage 4: Kontenrahmen für die Buchführung

- Anlage 5: Kostenstellen für die Kosten- und Leistungsrechnung.

$182 \mathrm{Zu}$ denen gehören nach $\S 5$ Abs. 1 KHG Krankenhäuser und Einrichtungen, die besondere Situationen oder Trägern haben. Beispielsweise Tuberkulose-Krankenhäuser, Versorgungskrankenhäuser und solche Einrichtungen, soweit sie durch besondere Bedürfnisse des Zivilschutzes bedingt sind. 


\section{Bundespflegesatzverordnung (BPflV)}

Die Verordnung zur Regelung der Krankenhauspflegesätze (BPflV) vom 21.08.1985, ${ }^{183}$ wurde am 20.09.94 und durch Artikel 12 des GSG für das Jahr 1995 geändert. Zuletzt wurde es geändert durch die fünfte Verordnung zur Änderung der Bundespflegesatzverordnung vom 09. 12. 1997. ${ }^{184}$

Die BPflV umfasst Regelungen für die Abgrenzung der pflegesatzfähigen Leistungen sowie Grundlagen der Entgeltbemessung, Vergütung der allgemeinen Krankenhausleistungen und Pflegesatzverfahren, ${ }^{185}$ d. h. die Verordnung nimmt indirekt ebenfalls Einfluss auf das Rechnungswesen, insbesondere die Kostenrechnung. ${ }^{186}$ Nach $\S 8$ Abs. 1 Satz 1 der KHBV muss die vom Krankenhaus zu führenden Kosten- und Leistungsrechnung auch die Ermittlung der Selbstkosten sowie die Erstellung des Kosten- und Leistungsnachweises nach den Vorschriften der BPflV ermöglichen.

\section{Abgrenzungsverordnung (AbgV)}

Die Bundesregierung hat mit Zustimmung des Bundesrates nach $\S 16$ Nr. 5 KHG die Verordnung über die Abgrenzung der im Pflegesatz nicht zu berücksichtigenden Investitionskosten von den pflegesatzfähigen Kosten der Krankenhäuser Abgrenzungsverordnung (AbgV) von 21. Dezember 1985 erlassen. ${ }^{187}$ Die letzte Änderung der AbgV wurde durch die Verordnung zur Neuordnung des Pflegesatzrechts von 26.09.1994durchgeführt. ${ }^{188}$

Diese Verordnung regelt die Abgrenzung und Zuordnung von Wirtschafts-, Gebrauchs- und Verbrauchsgüter und die Bestimmung der durchschnittlichen Nutzungsdauer der Wirtschaftsgüter in Krankenhäusern. ${ }^{189}$

\footnotetext{
183 BGB1. I, S. 1666.

184 BGB1. I, S. 2874.

185 Diese Verordnung gilt nicht für Krankenhäuser, auf die das Krankenhausfinanzierungsgesetz nach $\S 3$ Satz 3 Nr. 1 bis 4 keine Anwendung findet, und die nach $\S 5$ Abs. 1 Nr.2, 4 oder 7 des Krankenhausfinanzierungsgesetzes nicht gefördert werden, ( 1 Abs. 2 BPflV).

${ }^{186}$ Vgl. Fuchs, M. (1988), S. 68.

187 BGB1 I, S.2255.

188 BGBl I, S.2750.

189 Dieses Gesetz findet ebenfalls Anwendung bei allen Krankenhäusern, für die das KHG verpflichtend ist. Vgl. Fuchs, M. (1988), S. 66.
} 


\subsubsection{Begriffsdefinitionen zur Kosten- und Leistungsrechnung in Deutschland}

\subsubsection{Kostenbegriff}

Bezüglich des Aufbaus einer krankenhausbetrieblichen Kostenrechnung folgt der Gesetzgeber der pagatorischen Sichtweise des Kostenbegriffs. Diese Überlegung ergibt sich mittelbar aus spezifischen Rechtsvorschriften, die die Forderung nach einer nachprüfbaren Herleitung der Kosten aus der Finanzbuchhaltung und die positive Aufzählung von pflegesatzfähigen Kostenelementen beinhalten. Durch die BPflV wird der Wortlaut des $\S 8 \mathrm{KHBV}$ geändert in ,...die Ermittlung der pflegesatzfähigen Kosten sowie die Erstellung der Leistungs- und Kalkulationsaufstellung nach den Vorschriften der Bundespflegesatzverordnung.. “190. Der gesetzliche Kostenbegriff entspricht den pagatorischen Kosten, die den Grundkosten gleichgesetzt werden können. Darüber hinaus sind Abschreibungen auf Anlagegüter bei nicht oder teilweise geförderten Krankenhäusern entsprechend steuergesetzlichen Regelungen auf der Grundlage der pagatorischen Anschaffungsoder Herstellungskosten zu bilden. ${ }^{191}$

Da der Kostenbegriff des Krankenhausfinanzierungsrechts ein pagatorischer ist, der auf erfolgswirksame Ausgaben abstellt, bleiben kalkulatorische Kosten in der Kostenrechnung der Krankenhäuser meist unberücksichtigt. Das bedeutet, dass sich die Abgrenzung zwischen Aufwandsarten der Finanzbuchhaltung und Kostenarten auf die betriebsfremden Aufwendungen beschränkt. ${ }^{192}$ Z. B. entfällt der Ansatz von kalkulatorischen Abschreibungen und Zinsen wegen der Finanzierung der Investitionskosten. Betriebsmittelkreditzinsen hingegen beziehen sich nur auf budgetfähige und nicht auf investive Bestandteile und gehen als Kosten in die Kosten- und Leistungsrechnung ein.

Selbstkosten des Krankenhauses sind nach $§ 17$ Abs. 2 KHG die Kosten der Krankenhausleistungen, die nach Maßgabe der Krankenhausbuchführung auf der

\footnotetext{
190 Vgl. § 8 KHBV.

191 Vgl. Preuß, O. (1996), S.60-61.

192 Ausnahmeweise werden kalkulatorische Kosten bei den Kalkulationsverfahren zur Ermittlung der pauschalierten Entgelte und der krankenhausindividuellen Basispflegesätze nicht berücksichtigt. Vgl. Baugut, G., Schmitz, R. (1993): Methodik zur Kalkulation hausindividueller
} 
Grundlage der kaufmännischen Buchführung und einer Kosten- und Leistungsrechnung zu ermitteln sind.

Zum gesetzlichen Kostenbegriff gehören daher pflegesatzrelevante Kosten, also die Kosten, die bei Ermittlung des Pflegesatzes Berücksichtigung finden dürfen. ${ }^{193}$ Pflegesatzfähige Kosten sind nach $\S 2$ Nr. 5 KHG die Kosten des Krankenhauses, deren Berücksichtigung im Pflegesatz nicht nach diesem Gesetz ausgeschlossen ist.

Nach § 3 Abs. 1 Abgrenzungsverordnung (AbgV) sind pflegesatzfähig:

1. die Kosten der Wiederbeschaffung von beweglichen, selbständig nutzungsfähigen Gebrauchsgütern, deren Anschaffung- oder Herstellungskosten für das einzelne Gebrauchsgut ohne Umsatzsteuer 800,00 DM nicht übersteigen,

2. sonstige Investitionskosten und ihnen gleichstehende Kosten nach Maßgabe der $\S 17$ u. 18 des KHG und $\S 8$ BPflV,

3. die Kosten der Anschaffung oder Herstellung von Verbrauchsgütern sowie

4. Die Kosten der Instandhaltung von Anlagegütern nach Maßgabe des $§ 4 .{ }^{194}$

Die nicht pflegesatzrelevanten Kosten sind Krankenhauskosten, die bei der Ermittlung des Pflegesatzes nicht berücksichtigt werden dürfen. Zu diesen gehören bestimmte Investitionskosten, die die gemäß $§ 2$ Nr. 2 KHG aufgeführten Ausgaben sind, ${ }^{195}$ Kosten für Leistungen, die nicht der stationären oder teilstationären

Fallkosten für den Vergleich mit standardisierten Fallpauschalen, in: das Krankenhaus, 4/ 1993, S. 172.

193 Nach $§ 2$ Abs. 4 KHG sind Pflegesätze die Entgelte der Benutzer oder ihrer Kostenträger für stationäre und teilstationäre Leistungen des Krankenhauses. Die Kosten für allgemeine Krankenhausleistungen werden mit Pflegesätzen nur vergütet, wenn diese Kosten dem Grunde nach pflegesatzfähig sind. Vgl. §7 Abs.1 BPflV.

194 Kosten der Instandhaltung sind nach $\S 4 \mathrm{AbgV}$ die der Erhaltung oder der Wiederherstellung von Anlagegütern des Krankenhauses, wenn dadurch

i. das Anlagegut in seiner Substanz nicht wesentlich vermehrt, in seinem Wesen nicht erheblich verändert, seine Nutzungsdauer nicht wesentlich verlängert oder über seinen bisherigen Zustand hinaus nicht deutlich verbessert wird

ii. a) in baulichen Einheiten: Gebäudeteilen, betriebstechnische Anlagen und Einbauten oder

b) Außenanlagen

nicht vollständig oder nicht überwiegend ersetzt werden.

Für die Beurteilung des überwiegenden Ersatzes sind Maßnahmen, die im Rahmen eines einheitlichen Vorhabens in einem Zeitraum bis $\mathrm{zu}$ drei Jahren durchgeführt werden, zusammenzurechnen.

195 Die Finanzierung der Investitionsausgaben erfolgt über die Länder, d. h. sie dürfen nicht über die Pflegesätze dem Krankenhaus zufließen. Für die im Pflegesatz zu berücksichtigenden 
Krankenhausversorgung dienen, ${ }^{196}$ und Kosten für wissenschaftliche Forschung und Lehre, die über den normalen Krankenhausbetrieb hinausgehen.

Gemäß 33 Abs. 2 AbgV sind nicht pflegesatzfähig:

1. Kosten :

a. der Errichtung und Erstausstattung von Krankenhäusern mit Ausnahme der Kosten nach Absatz 1 Nr. 3,

b. der Ergänzung von Anlagegütern, soweit diese über die übliche Anpassung der vorhandenen Anlagegüter an die medizinische und technische Entwicklung wesentlich hinausgehen,

2. die Kosten der Wiederbeschaffung von Anlagegütern mit einer durchschnittlichen Nutzungsdauer von mehr als drei Jahren und

3. die Kosten der Erhaltung oder Wiederherstellung von Anlagegütern, soweit diese Kosten nicht zu den Instandhaltungskosten nach $§ 4$ gehören.

Tab. 2 stellt die Kosten des Krankenhauses mit der gesetzlichen Abfassung in pagatorischen Kosten (Pflegesatzrelevante-, und Investitionskosten) und kalkulatorischen Kosten dar:

\begin{tabular}{||l|l|l|l||}
\hline \multirow{2}{*}{ Kostenarten } & \multicolumn{2}{|l||}{ Pagatorische Kosten } & Kalkulator- \\
\cline { 2 - 4 } & $\begin{array}{l}\text { Pflegesatz- } \\
\text { relevante } \\
\text { Kosten }\end{array}$ & $\begin{array}{l}\text { Investit- } \\
\text { ions } \\
\text { kosten }\end{array}$ & Kosten \\
\hline Löhne u. Gehälter & $\mathrm{x}$ & & \\
\hline Gesetzliche Sozialabgaben & $\mathrm{x}$ & & \\
\hline Aufwendung für Altersversorgung & $\mathrm{x}$ & & \\
\hline $\begin{array}{l}\text { Aufwendung für Beihilfen und Unter- } \\
\text { stützung }\end{array}$ & $\mathrm{x}$ & & \\
\hline Sonstige Personalaufwendungen & $\mathrm{x}$ & & \\
\hline $\begin{array}{l}\text { Lebensmittel und bezogene } \\
\text { Leistungen }\end{array}$ & $\mathrm{x}$ & & \\
\hline
\end{tabular}

Investitionskosten gelten durch das GSG neue Regelungen. Neben den für die Wiederbeschaffung von Wirtschaftsgütern mit einer durchschnittlichen Nutzungsdauer von bis zu drei Jahren sind auch die Ausgaben der Finanzierung von Rationalisierungsinvestitionen nach §18b KHG nicht zu den Investitionskosten zu rechnen und damit von den pflegesatzfähigen Kosten ausgeschlossen. Vgl. Keun, F. (1997), S.38-39.

$196 \$ 17$ Abs. 3 Nr.1 KHG 


\begin{tabular}{|c|c|c|c|}
\hline Medizinischer Bedarf & $\mathrm{x}$ & & \\
\hline Wasser, Energie, Brennstoffe & $\mathrm{x}$ & & \\
\hline Wirtschaftsbedarf & $\mathrm{x}$ & & \\
\hline $\begin{array}{l}\text { Verwaltungsbedarf } \\
\text { - } \text { ADV- } \\
\quad \text { Organisationsaufwand } \\
\text { - } \text { Fernsprechanlagen }\end{array}$ & $\begin{array}{l}\text { teilweise } \\
\text { teilweise }\end{array}$ & $\begin{array}{l}\text { teilweise } \\
\text { teilweise }\end{array}$ & \\
\hline Wiederbeschaffte Gebrauchsgüter & $\mathrm{x}$ & $\mathrm{x}$ & \\
\hline $\begin{array}{l}\text { Instandhaltung } \\
\text { - finanziert nach } § 7 \text { Abs.1 Nr. } 4 \\
\text { BPflV } \\
\text { - nicht aktivierungsfähige nach dem } \\
\text { KHG } \\
\text { geförderte Maßnahmen }\end{array}$ & $\mathrm{x}$ & $\mathrm{x}$ & \\
\hline Steuern, Abgaben, Versicherungen & $\mathrm{x}$ & & \\
\hline $\begin{array}{l}\text { Zinsen und ähnliche Aufwendungen } \\
\text { für Betriebsmittelkredite }\end{array}$ & $\mathrm{x}$ & & \\
\hline $\begin{array}{l}\text { Auflösung von Ausgleichsposten und } \\
\text { Zuführung der Fördermittel nach } \\
\text { KHG zu Sonderposten oder } \\
\text { Verbindlichkeiten }\end{array}$ & & $\mathrm{x}$ & \\
\hline Abschreibungen & teilweise & teilweise & \\
\hline $\begin{array}{l}\text { Zustandkosten } \\
\begin{aligned}- & \text { kalk. Unternehmerlohn } \\
- & \text { kalk. Eigenkapitalzinsen } \\
- & \text { kalk. Eigenmiete }\end{aligned}\end{array}$ & & & $\begin{array}{l}\mathrm{x} \\
\mathrm{x} \\
\mathrm{x}\end{array}$ \\
\hline $\begin{array}{l}\text { Anderskosten } \\
\text { kalkulatorische Abschreibungen } \\
\text { kalkulatorische Zinsen } \\
\text { kalkulatorische Wagnisse }\end{array}$ & & & $\begin{array}{l}\mathrm{x} \\
\mathrm{x} \\
\mathrm{x}\end{array}$ \\
\hline
\end{tabular}

Tab. 2: Abgrenzung von pagatorischen und kalkulatorischen Kosten ${ }^{197}$

\subsubsection{Leistungsbegriff}

$\mathrm{Zu}$ den Zwecken der Kosten- und Leistungsrechnung wurden die Krankenhausleistungen vom deutschen Gesetzgeber definiert. Danach sind Krankenhausleistungen die folgenden: ${ }^{198}$

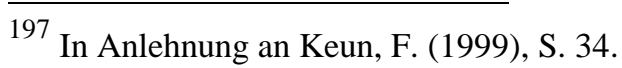


1. Krankenhausleistungen nach $\S 1$ Abs. 1 sind insbesondere ärztliche Behandlung, Krankenpflege Versorgung mit Arznei-, Heil-, und Hilfsmitteln, die für die Versorgung im Krankenhaus notwendig sind, sowie Unterkunft und Verpflegung, sie umfassen allgemeine Krankenhausleistungen und Wahlleistungen. $\mathrm{Zu}$ den Krankenhausleistungen gehören nicht die Leistungen der Belegärzte (§ 23) sowie der Beleghebammen und -entbindungspfleger.

2. Allgemeine Krankenhausleistungen sind diejenigen, die unter Berücksichtigung der Leistungsfähigkeit des Krankenhauses im Einzelfall nach Art und Schwere der Krankheit für die medizinisch zweckmäßige und ausreichende Versorgung des Patienten notwendig sind. Unter diesen Voraussetzungen gehören dazu auch,

a. die während des Krankenhausaufenthalts durchgeführten Maßnahmen zur Früherkennung von Krankheiten im Sinne des SGB V,

b. die vom Krankenhaus veranlassten Leistungen Dritter,

c. die aus medizinischen Gründen notwendige Mitaufnahme einer Begleitperson des Patienten sowie

d. die besonderen Leistungen von Tumorzentren und onkologischen Schwerpunkten für die stationäre Versorgung von krebskranken Patienten.

Der vom Gesetzgeber gewählte Leistungsbegriff dient vor allem der Kostenbegrenzung verschiedener „Geldtöpfe“, wie z. B. für die stationäre und ambulante Krankenhausleistungen. Was darf in das Budget für die stationäre Versorgung hineingerechnet werden und was nicht? ${ }^{199}$

Die Krankenhausleistungen werden nach der Art der Abrechnung unterschieden in Allgemeine- und Wahlleistungen. Für Wahlleistungen, z. B. für Zwei- oder Einbettzimmer oder Telefon darf ein deutlich höheres und nicht unbedingt mittels nachweisbarer Kosten kalkuliertes Entgelt festgesetzt werden. Eine inhaltliche Spezifizierung von Leistungsarten erfolgt nur für einen Teil des gesamten

\footnotetext{
198 Vgl. §2 Abs. 1, 2 BPflV.

199 Vgl. Grünenwald, K., Kehr, H., Tuschen, K. (1987): Kommentar Leistungs- und Leistungsnachweis der Krankenhäuser, Bad Homburg, 1987, S. 274.
} 
Leistungsspektrums des Krankenhauses im Zusammenhang mit dem Kosten- und Leistungsnachweis gemäß $§ 16$ Abs. 4 BPflV. ${ }^{200}$

\subsubsection{Mindestanforderungen an die Kosten- und Leistungsrechnung im deut- schen Krankenhaus}

Die Durchführung der Kosten- und Leistungsrechnung in den deutschen Krankenhäusern ist nicht nur aus Gründen der allgemeinen betrieblichen Funktionen sinnvoll, sondern auch aus Gründen der Erfüllung von Vorschriften der Gesetze und Verordnungen.

Nach speziellen Gesetzen ${ }^{201}$ muss das Krankenhaus Mindestanforderungen an die Kosten- und Leistungsrechnung erfüllen. Aufgaben und grundlegender Aufbau der Kosten- und Leistungsrechnung im deutschen Krankenhaus sind in der Krankenhausbuchführungsverordnung (KHBV) auf der Grundlage des Krankenhausfinanzierungsgesetzes (KHG) sowie der Bundespflegesatzverordnung (BPflV) konkretisiert.

Gemäß $\S 8$ Abs. 1 KHBV hat jedes Krankenhaus die Pflicht, eine aus der Buchführung nachprüfbar herzuleitende Kosten- und Leistungsrechnung zu führen. Die Befreiung von der Pflicht zur Führung einer Kosten- und Leistungsrechnung ist möglich, wenn ein Krankenhaus über weniger als 100 Betten verfügt oder nur eine bettenführende Abteilung aufweist, die Kosten für die Einrichtung der Betriebsbuchhaltung in einem unangemessenen Verhältnis zu dem erreichbaren Nutzen stehen und die nach $\S 8$ Abs. 1 KHBV geforderten Zwecke durch Betriebsstatistiken oder Sonderrechnungen erreicht werden können. ${ }^{202}$

Die Einführung der neuen Entgeltformen bedeutet für die meisten deutschen Krankenhäuser aber zumindest die Einrichtung einer differenzierteren Leistungserfassung sowie die Notwendigkeit der Erweiterung des bisherigen Kostenrechnungssystems um eine aussagefähige Kostenträgerrechnung. ${ }^{203}$

\footnotetext{
200 Vgl. Hildbrand, R. (1988), S.381.

${ }^{201} \mathrm{Vgl}$. Abschnitt 3.2.2.2.

202 Vgl. §9 KHBV (Befreiungsvorschrift).

203 Vgl. Maltry, H., Strehlau-Schwoll, H. (1997): Kostenrechnung und Kostenmanagement im Krankenhaus, in: Freidank, C., Götze, U., Huch, B., Weber, J. (Hrsg.): Kostenmanagement, neue Konzepte und Anwendungen, Berlin, Heidelberg, 1997, S. 536.
} 
Gemäß $§ 17$ Abs. 2 KHG sind die Kosten der Krankenhausleistungen nach Maßgabe der Krankenhaus-Buchführungsverordnung auf der Grundlage der kaufmännischen Buchführung und einer Kosten- und Leistungsrechnung zu ermitteln und gemäß $\S 8$ Nr. 2 KHBV nachprüfbar herzuleiten.

Nach $\S 17$ Abs. 4 BPflV 1995 ist festgelegt, anstelle des Kosten und Leistungsnachweises eine Leistungs- und Kalkulationsaufstellung nach dem Muster der Anlage 3 und 4 der BPflV nur noch auf Verlangen einer Vertragspartei zu erstellen, d. h. der Nachweis der Selbstkosten ist nicht mehr zwingend erforderlich, eine Konsequenz aus dem aufgehobenen Selbstkostendeckungsanspruch. ${ }^{204}$

$\S 8 \mathrm{KHBV}$ stellt an die Kosten- und Leistungsrechnung der deutschen Krankenhäuser folgende Mindestanforderungen:

Das Krankenhaus hat die aufgrund seiner Aufgaben und Struktur erforderlichen Kostenstellen zu bilden. Es sollen, sofern hierfür Kosten und Leistungen anfallen, mindestens die Kostenstellen gebildet werden, die sich aus dem Kontenrahmen, der Anlage der KHBV ist, ergeben. Bei abweichender Gliederung dieser Kostenstellen soll durch ein ordnungsmäßiges Überleitungsverfahren die Umschlüsselung auf den Kontenrahmen sichergestellt werden.

Die Kosten sind aus der Finanzbuchhaltung nachprüfbar herzuleiten.

Die Kosten und Leistungen sind verursachungsgerecht nach Kostenstellen zu erfassen. Sie sind darüber hinaus den anfordernden Kostenstellen zuzuordnen, soweit dieses für die Zwecke und für die Erstellung des Kosten- und Leistungsnachweises erforderlich ist.

Der Schwerpunkt der Kosten- und Leistungsrechnung im Krankenhaus liegt damit in der Kostenstellenrechnung. Jedoch wird auch eine Kostenträgerrechnung ausdrücklich $\S 8 \mathrm{KHBV}$ verlangt. Diese erfolgt hinsichtlich der stationären Krankenhausleistungen im Kosten- und Leistungsnachweis außerhalb des Systems der Kostenrechnung. Dort wird auf der Grundlage des Budgets und der voraussichtlichen Belegung für jedes Krankenhaus ein allgemeiner Pflegesatz ermittelt.

Ausgehend vom Wortlaut des $\S 8 \mathrm{KHBV}$ genügt es allerdings nicht, dass die Kosten- und Leistungsrechnung nur gemäß den gestellten Mindestanforderungen

${ }^{204}$ Vgl. Keun, F. (1997), S. 33. 
durchgeführt wird, um ihre Aufgaben als Instrument zur betriebsinternen Steuerung und Wirtschaftlichkeitskontrolle voll zu erfüllen. ${ }^{205}$

\subsection{Grundlagen der Kosten- und Leistungsrechnung in syrischen Kranken- häusern}

\subsubsection{Rechtliche Rahmenbedingungen der Kosten- und Leistungsrechnung in syrischen Krankenhäusern}

Als rechtlicher Rahmen steht grundsätzlich das Gesetz für das Syrische Eeinheitliche Rechnungs-System (SERS). Es trat am 19.03.1974 in Kraft und wurde bisher nur einmal am 25.01.1978 geändert. ${ }^{206}$

SERS ist die einzige rechtliche Rahmenbedingung für die Kosten- und Leistungsrechnung, nach deren Regelungen die Betriebe ihre Aktivitäten verrechnen müssen. Nach $\S 1$ SERS gelten die Vorschriften des Gesetzes besonders an Betriebe und Betriebsgruppen des "Public-Sektors"207 in Syrien außer Banken- und Versicherungsbranchen. Dies umfasst die Betriebe der Industrie, des national oder international Handels, der Dienstleistung und der Landwirtschart.

Da die Betriebe des Public-Sektors die größten und relevantesten in Syrien sind, gilt das SERS in der Regel für einen großen Teil der syrischen Wirtschaft, mit dem Ziel, die Privatwirtschaft anzuspornen. ${ }^{208}$ In der Praxis ist das SERS tatsächlich schon von allen industriellen Betrieben, aber nicht von vielen der Dienstleistungsbetriebe (z. B. Krankenhäuser) angewendet worden. Trotzdem bleibt das SERS eine rechtliche Rahmenbedingung für eventuelle Durchführung sowie Entwicklung einer Kosten- und Leistungsrechnung in den syrischen Krankenhäusern.

\footnotetext{
205 Vgl. Abschnitt 5.2.

206 Vor 1974 gab es in Syrien keine gesetzlichen Vorschriften, die die Kosten- und Leistungsrechnung in den Betrieben regelten. Die Betriebe hatten nach eigenem Wunsch und gemäß allgemeiner Rechnungswesen- Standards in der Praxis ihre Kostenrechnung durchgeführt.

207 Betriebe und Betriebsgruppen des "Public-Sektors" sind Organisationen, die eigene juristische und administrative eigenständige Personalität im Rahmen der staatlichen Wirtschaft haben. Sie betrieben eine industrielle, agrarwirtschaftliche, finanzielle sowie Handels- Anstrengung. § 1 Abs. 3 Grund Finanzierungsgesetz Nr. 92/1967.

208 Sakka, H. (1998): das einheitliche Rechnungssystem, 10 Aufl., Universität Damaskus (Hrsg.), Damaskus, 1998, S. 88.
} 


\subsubsection{Aufgaben der Kosten- und Leistungsrechnung in syrischen Kranken- häusern}

Da die Kosten- und Leistungsrechnung als Subsystem des gesamten Rechnungssystems in Syrien betrachtet wird, musste sie in der ersten Reihe die Hauptaufgabe des SERS erfüllen. Die Kosten- und Leistungsrechnung nach dem SERS hat externe sowie interne Aufgaben zu erfüllen:

1. Externe Aufgaben: Die Hauptaufgabe der Kosten- und Leistungsrechnung in syrischen Betrieben besteht darin, der zentralen staatlichen Einrichtungen (die Regierung), die für die zentrale Planung der gesamten Nationalwirtschaft verantwortlich sind, mit ausreichenden Angaben über die Betriebe zu versorgen.

Die Angaben sollen alle Einzelheiten über alle Betriebe erhalten, die homogenen nach den einheitlichen Vorschriften des SERS verrechnet werden. ${ }^{209}$

2. Nach $\S 11$ Abs. 1 SERS hat die Kosten- und Leistungsrechnung folgenden interne Aufgaben zu erfüllen:

a. Erfassung und Verrechnung der Kosten und Leistungen,

b. Analyse und Kontrolle der Kosten der Kostenstellen,

c. Bestimmung der Kosten der Leistungseinheit und

d. Darstellung der Ist- sowie Normal-Kosten in periodischen Kostenberichten. $^{210}$

Diese Aufgaben sollen auch von der Kosten- und Leistungsrechnung in syrischen Krankenhäusern erfüllt werden, womit zum einen ausreichende Informationen über die Kosten und Leistungen des Krankenhauses dem Ministerium für Gesundheit mitgeteilt werden können, das Planungen für das Krankenhaus in Rahmen der staatlichen Gesundheitspolitik durchführt (externe Aufgaben). Zum anderen dient die Kosten- und Leistungsrechnung als Instrument zur Erfassung, Verrechnung und Kontrolle der Kosten im Krankenhaus (interne Aufgaben). Und solange keine

\footnotetext{
${ }^{209}$ Das SERS hat einheitliche Begriffsdefinitionen sowie einheitliche Verfahren zum Verrechnungsund Versammlungsprozess, um homogene Daten über verschiedenen Wirtschaftsbereiche und -betriebe der zentralen Nationaleinrichtungen versorgen zu können.
} 
Kosten- und Leistungsrechnung in Krankenhäusern durchgeführt wird, steht der staatlichen Zentraleinrichtungen keine die bestimmten und definierten Angaben über alle Krankenhäuser, die zur Planung unabdingbar sind. Die KrankenhausLeitung verliert ebenfalls das benötigte Instrument für Planung, Steuerung und Kontrolle des betrieblichen Geschehens.

\subsubsection{Begriffsdefinition zur Kosten- und Leistungsrechnung in syrischen Krankenhäusern}

Da das SERS die Rechnungsverfahren in allen Betriebe vereinheitlicht, um das Ziel von homogener Daten-Zusammenstellung aller wirtschaftlichen Branchen $\mathrm{zu}$ erreichen, hat der syrischen Gesetzgeber detaillierte Definitionen für die meisten rechnungswesenbezogenen Begriffe ${ }^{211}$.

Begriffsdefinitionen, die sich auf die Kosten- und Leistungsrechnung beziehen, werden in Folgenden dargestellt.

\subsubsection{Der Kostenbegriff}

Der syrischen Gesetzgeber hat die wertmäßige Sichtweise des Kostenbegriffs herangezogen, da die Kosten- und Leistungsrechnung nach dem SERS die Kosten aller Betriebe in erster Linie der Berechnung der Nationalprodukts dienen soll. Mit dieser Aufgabe muss in der Kosten- und Leistungsrechnung alle wertmäßigen Kosten erfasst werden.

Die Kosten der Leistungen nach SERS sind alle Kosten, die direkt oder indirekt der Leistungseinheit in den Produktionskostenstellen zugeordnet werden. Werden dazu die Vertriebskosten verrechnet, resultiert die Umsatzkosten, mit Einordnung der administrativen Kosten dazu resultiert die sämtlichen Kosten der Leistungen. ${ }^{212}$ Analog dazu werden mit Zurechnung der direkten und indirekten Kosten, welche die Leistungen einer Krankenhaus-Abteilung bezogen, resultiert die

\footnotetext{
210 Die Kostenberichte sollen regelmäßig alle 3 Monaten erstellt werden. Sie sollen Angaben über Ist-Kosten der aktuellen und vergangenen Perioden erhalten. Die Abweichungen sollen ebenfalls bestimmt und analysiert werden. $\$ 62$ A und B SERS.

211 SERS erfasst auch bestimmte Definitionen für Begriffe wie z. B. Vermögen, Liquidität, Eigenoder Fremdkapital, Produktion sowie Abschreibung.

${ }^{212}$ Vgl. § 3 Abs. 31- 33 SERS.
} 
Pflegesatzkosten. Ordnet dazu die administrativen Kosten ergeben sich die sämtlichen Kosten der Krankenhausleistungen.

Ist- und Normal-Kosten: Istkosten sind diejenigen, die sich aus der Zurechnung aller angefallenen Kosten der Leistungen der vergangenen Periode erbeben. Normalkosten sind diejenigen, die sich in einer Periode nach technischer Analyse und Bestimmung des Produktionsprozesses jeder Leistung ergeben. Mit der Vergleich zwischen Ist- und Normal-Kosten können die Abweichungen bestimmt und analysiert werden. ${ }^{213}$

Direkte- und Gesamt- Kosten: Direkte Kosten sind diejenigen, die zu einer Leistungseinheit oder Kostenstelle direkt zurechnet werden können. Sie umfassen direkte Material, direkte Personal-Kosten und direkte Kosten der ProduktionsHilfsstellen. Gesamtkosten sind diejenige, die zur Leistungseinheit oder Kostenstelle direkt nicht zurechnfähig sind. Sie sollen indirekt nach bestimmten Schlüsseln (Zeit, Löhne oder Fläche) umgerechnet werden. ${ }^{214}$

Fixe und variable Kosten: Die Fixkosten entstehen in bestimmten Periode nach vorausbestimmten Produktions- oder Vertriebs-Kapazität, aber sie ändern sich nicht mit der Änderung der Produktionsmenge. Meistens liegen sie außerhalb der Managemententscheidungen wie z. B. Miete, Versicherungen oder gesetzlichen Steuern und Ausgaben. Variable Kosten sind diejenige, die zur Produzierung sowie Vertrieb eine Leistung benötigt sind, aber sie ändern sich proportional mit der Änderung von Produktionskapazität wie oft die Produktionsbedingungen von Materialpreise, Löhne und technischer Prozesse sich nicht geändert haben. Lassen sich solche Fixkosten bei der Änderung des Beschäftigungsniveaus sprunglich ändern, handelt sich hierbei an Sprung-Fixkosten. ${ }^{215}$

\subsubsection{Der Leistungsbegriff:}

Der syrische Gesetzgeber hat die Leistungen nach ihren Komponenten definiert. Leistungen können eine oder mehre von folgenden Elemente enthalten: ${ }^{216}$

\footnotetext{
213 Vgl. § 3 Abs. 34- 35 SERS.

214 Vgl. § 3 Abs. 18 und Abs. 23 SERS.

215 Vgl. § 3 Abs. 27-29 SERS.

${ }^{216}$ Vgl. § 6 Abs. 4 SERS.
} 
1. Der Wert der hergestellten und vertriebenen Produkte oder Dienstleistungen.

2. Die positiven Bestandsveränderungen der zwischen- und Fertigprodukte. Diese sollen in erster Stufe für die Zwecke der Nationalverrechnung nach Marktpreise bewertet werden und dann für die Zwecke der Kosten- und Leistungsrechnung nach den Herstellungskosten bewertet werden, falls ihre Kosten niedriger als der Marktpreis sind. ${ }^{217}$

3. Innerbetriebliche Leistungen für eigene Verwendung wie z.B. selbst Herstellung von Tools oder Geräte, die vom Betrieb selbst genutzt werden.

4. Leistungen für Dritten.

Entsprechend entsteht bei der Leistungsdefinition im Krankenhaus nach den Regelungen des SERS eine große Komplexität. Insbesondere ergibt sich die Schwierigkeit im Krankenhaus, wenn am Ende einer Periode der Gesundheitszustand von Patienten, die noch nicht entlassen wurden, im Sinne von „Zwischenprodukten“ bewertet werden sollen, um in der Nationalverrechnung Berücksichtigung zu finden.

\section{TEILGEBIETE DER KOSTEN- UND LEISTUNGSRECHNUNG}

\subsection{Aufbau der Kosten- und Leistungsrechnung im Krankenhaus}

Die Grundaufbau der Kostenrechnung besteht aus drei Stufen: Kostenartenrechnung, Kostenstellenrechnung und Kostenträgerrechnung, die im Folgenden kurz allgemein erläutert werden sollen.

Zu Darstellung des Aufbaus einer Krankenhaus-Kostenrechnung müssen zunächst Kostenarten-, Kostenstellen-, Bezugsgrößen- und Kostenträgerrechnung erstellt werden sowie die Art der technischen Durchführung der Kostenrechnung bzw. ihrer Integration in das Krankenhaus- Rechnungswesen geklärt werden. Dieser Prozess wird in Abb.14 dargestellt.

217 Aus der Sicht der Nationalverrechnung sind Produkte nach dem Marktpreis zu zurechnet werden, unterschiedslos ob sie schon vertrieben werden oder nicht. Dagegen der Wert der noch nicht vertriebenen Leistungen soll nach dem niedrigeren Wert von ihrem Marktpreis oder angefallenen. Kosten. Vgl. Sakka, H. (1997), S. 203. 


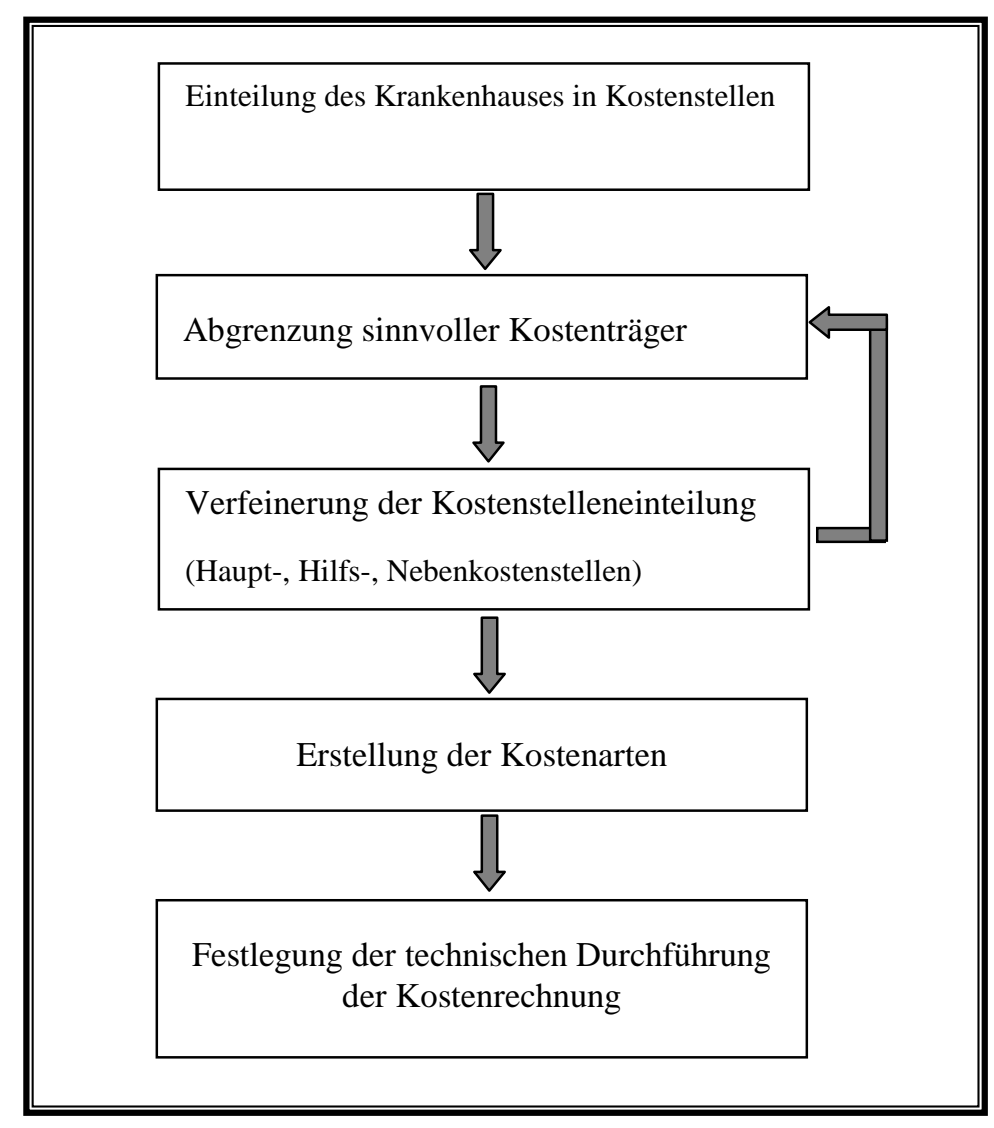

Abb. 14: Erstellung der Aufbauorganisation einer KrankenhausKostenrechnung $^{218}$

- Die Kostenartenrechnung erfasst alle im Laufe einer Abrechnungsperiode angefallenen bzw. anfallenden Kosten in systematisch gegliederter Form. Die Kostenartenrechnung beantwortet die Frage: Welche Kosten sind angefallen?

- In der Kostenstellenrechnung werden die einzelnen Kostenarten den Leistungsbereichen (Kostenstellen) zugeordnet, in denen sie entstehen sind bzw. durch die sie verursacht werden. Die Kostenstellenrechnung beantwortet die Frage: Wo sind die Kosten angefallen? Kostenarten- und Kostenstellenrechnung werden häufig unter dem Oberbegriff Betriebsrechnung zusammengefasst.

- In der Kostenträgerrechnung als dritte Stufe der Kostenrechnung werden die anfallenden Kosten auf die Kostenträger verteilt, nach dem sie in der Kostenartenrechnung erfasst und in der Kostenstellenrechnung auf die Endkostenstellen verrechnet worden sind. Die Kostenträgerrechnung beantwortet die 
Frage: Wofür sind welche Kosten in verschiedenen Kostenstellen in welcher Höhe entstanden? In der Kostenträgerrechnung kann man zwischen Kostenträgerzeitrechnung und Kostenträgerstückrechnung unterscheiden.

- Bei der Kostenträgerzeitrechnung werden die in einer Rechnungsperiode angefallenen Kostenträgerkosten den Leistungen (Erlöse) der Kostenträger gegenübergestellt. Die zumeist kurzfristige Rechnung ermittelt den Saldo zwischen diesen Größen und damit den Betriebserfolg.

- Bei der Kostenträgerstückrechnung werden die Kosten je Leistungseinheit ermittelt. $^{219}$

Die Kostenermittlung in den Stufen Kostenarten-, Kostenstellen- und Kostenträgerrechnung erfolgt rechnungstechnisch in den Phasen Kostenerfassung und Kostenverteilung ${ }^{220}$. Das stellt in Abb. 15 dar.

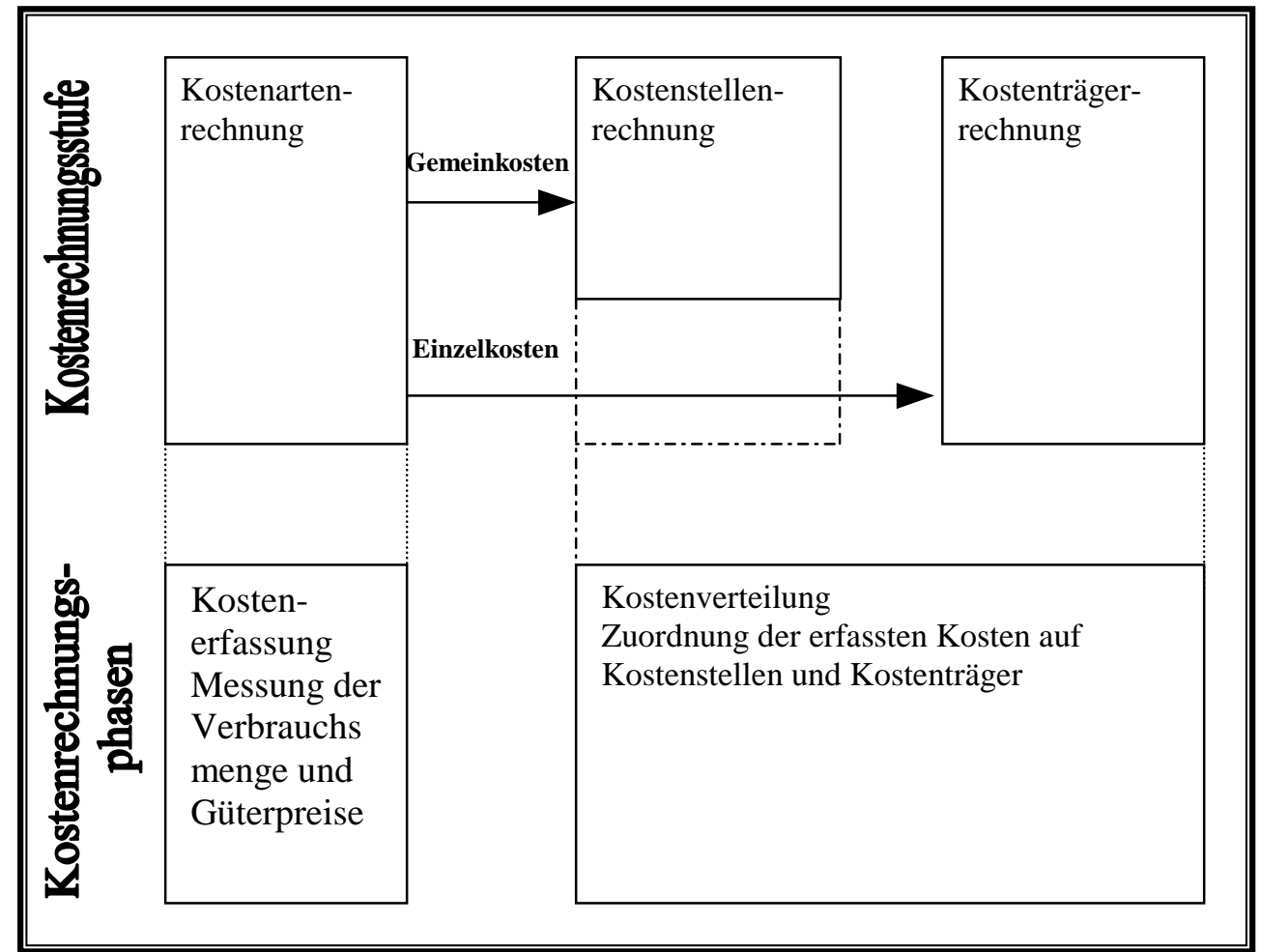

Abb. 15: Stufen und Phasen der Kostenrechnung 221

218 Quelle: Leonhardt, J. (1988), S.78.

219 Vgl. Haberstock, L. (1998): Kostenrechnung I, Einführung mit Fragen, Aufgaben einer Fallstudie und Lösungen, 10. Aufl., Berlin, 1998, S. 9-10.

${ }^{220}$ Vgl. Schweitzer, M., Küpper, H. U. (1995), S. 59.

${ }^{221}$ Quelle: Schweitzer, M., Küpper, H. U. (1995), S. 59. 


\subsubsection{Kostenartenrechnung}

\subsubsection{Definition und Aufgaben der Kostenartenrechnung}

Die Aufgabe der Kostenartenrechnung steht darin, den sachzielbezogenen Güterverbrauch mengen- und wertmäßig systematisch nach Kostenarten getrennt zu erfassen.

Die Kostenartenrechnung bildet die Grundlage der laufenden Kostenrechnung und dient einer systematischen Erfassung und Kategorisierung des in einer bestimmten Periode angefallenen sachzielbezogenen Güterverbrauchs nach Arten von Kostengütern. ${ }^{222}$ Sie erweist eine vollständige Erfassung und Darstellung der angefallenen Kosten durch die Leistungserstellung, insbesondere auch die Abgrenzung von zwischen Aufwendungen und Kosten. ${ }^{223}$

Die Kostenartenrechnung muss in zweckdienlicher, übersichtlicher und nachvollziehbarer Form angeben, welche Kosten im Krankenhaus entstanden sind bzw. entstehen werden. Damit ermittelt sie die Kosten als Grundlage für die Kostenstellen- und Kostenträgerrechnung.

Um eine vollständige und einheitliche Erfassung der Kosten zu ermöglichen, bedarf es zunächst eine Einteilung bzw. Gliederung der Kostenarten. ${ }^{224}$ Die Gliederung in Kostenarten hat so zu erfolgen, dass die Erreichung der Rechnungsziele durch die Kostenartenrechnung vorbereitet oder bereits realisiert wird. Die Erfassung der Kostenarten wird in Zusammenarbeit mit der Finanzbuchhaltung sowie vorgelagerten Rechnungskreisen wie der Lohn- und Gehaltsabrechnung und der Materialabrechnung vorgenommen. ${ }^{225}$

Bei der Kostenartenrechnung geht es darum, die einzelnen Kostenarten zu analysieren. Durch die Bestimmung prozentualer Anteile einzelner Kostenarten an den Gesamtkosten einer Periode ermöglicht sie einen schnellen Einblick in die Struktur eines Unternehmens und erlaubt damit eine erste Kostenanalyse, wobei die Entwicklung einzelner Kostenarten im Zeitablauf in Form von Zeitvergleichen anhand der absoluten Höhe oder des relativen Anteiles an den Gesamtkosten analysiert wird. Sie dient damit zur Kontrolle der absoluten Höhe einzelner

\footnotetext{
222 Vgl. Gabele, E. (1992): Kosten- und Erlösrechnung, München 1992, S. 62.

223 Vgl. Wahl, O. (1989): Kostenrechnung II für Studium und Praxis, Ist- und Normalkostenrechnung, 3., überarb. Aufl. Bad Homburg, 1989, S. 14.

${ }^{224}$ Vgl. Schweitzer, M., Küpper, H. U. (1995), S. 97.
} 
Kostenarten im Zeitvergleich sowie zur Kontrolle des relativen Anteils einzelner Kostenarten an den Gesamtkosten.

\subsubsection{Gliederung der Kostenartenrechnung}

Die Gliederung der Kostenartenrechnung wird von den Rechnungszielen der anschließenden Kostenstellen- und Kostenträgerrechnung stark beeinflußt. Die Kostenarten allein liefern zum Teil nicht immer aussagekräftige Informationen. Daher werden die in einem Abgrenzungszeitraum anfallenden Kosten je nach Ausrichtung der zu liefernden Informationen nach verschiedenen Kriterien gegliedert.

Die Kostenarten lassen sich nach verschiedenen Merkmalen und Kriterien systematisieren und bilden, die aber keineswegs alle für die Kostenartenrechnung anwendbar oder zweckmäßig sind. Die Kriterien, nach denen die Kostenarten sich bilden lassen, und resultierenden Einteilungen sind in Tab. 3 dargestellt.

\begin{tabular}{|c|c|}
\hline Einteilungskriterien & Kostenarten \\
\hline $\begin{array}{l}\text { Art der verbrauchten } \\
\text { Kostengüter }\end{array}$ & 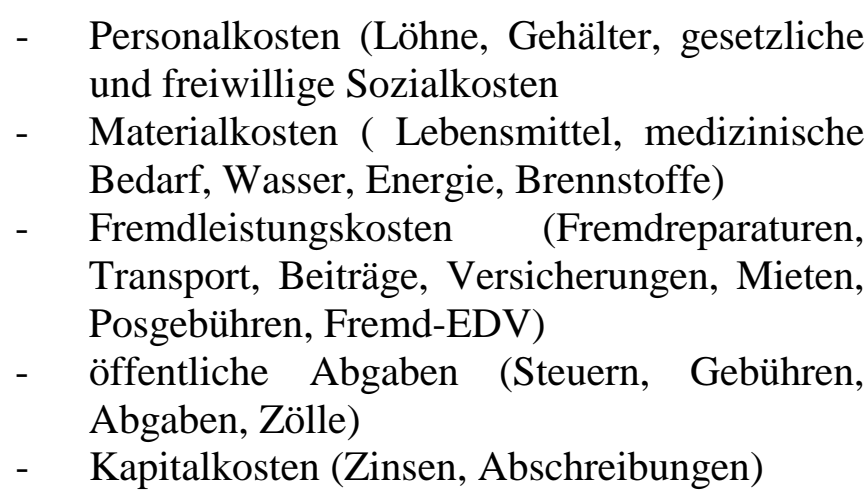 \\
\hline Art der Kostenerfassung & $\begin{array}{ll}- & \text { aufwandsgleiche Kosten } \\
- & \text { aufwandsungleiche Kosten }\end{array}$ \\
\hline Funktionsbereiche & $\begin{array}{ll}- & \text { Kosten der Verwaltung } \\
- & \text { Kosten der Versorgung } \\
- & \text { Kosten der Diagnostik, Therapie } \\
- & \text { Kosten der Pflege } \\
- & \text { Kosten für Sonstiges (z. B. Ausbildung }\end{array}$ \\
\hline
\end{tabular}

225 Vgl. Hummel, S., Männel, W. (1986), S. 14. 


\begin{tabular}{|c|c|}
\hline Herkunft der Kosten & $\begin{array}{ll}\text { - } & \text { primäre Kosten } \\
\text { - } & \text { sekundäre Kosten }\end{array}$ \\
\hline $\begin{array}{l}\text { Art der Verrechnung auf } \\
\text { Zurechnungsobjekte }\end{array}$ & $\begin{array}{ll}\text { - } & \text { Einzelkosten } \\
\text { - } & \text { Gemeinkosten } \\
\text { - } & \text { Unechte Gemeinkosten }\end{array}$ \\
\hline $\begin{array}{l}\text { Abhängigkeit von der } \\
\text { Leistungsmenge }\end{array}$ & $\begin{array}{ll}\text { - } & \begin{array}{l}\text { beschäftigungsabhängige } \\
\text { (proportionale, degressive, progressive }\end{array} \\
\text { Kosten) } \\
\text { - } \begin{array}{l}\text { beschäftigungstunabhängige Kosten (absolut } \\
\text { fixe, sprungfixe Kosten) }\end{array}\end{array}$ \\
\hline Ausgabewirksamkeit & 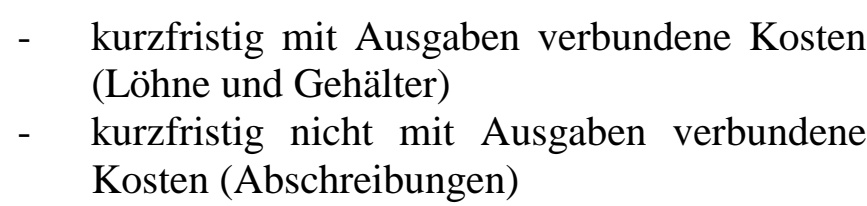 \\
\hline
\end{tabular}

Tab. 3: Bildungs- und Einteilungsmöglichkeiten der Kosten ${ }^{226}$

- Gliederung nach Art der verbrauchten Produktionsfaktoren: Nach diesem Kriterium werden Kostenarten gemäß Auskunft über die Art der Güter und des Dienstleistungsverzehrs in der Abrechnungsperiode eingeteilt in:

- Personalkosten (z. B. Löhne, Gehälter und Sozialabgaben),

- Sachkosten (z. B. Lebensmittel, medizinischer Bedarf, Wirtschaftsbedarf und Verwaltungsbedarf) und

- die Kapitalkosten (z. B. Zinsen für Betriebsmittelkredite).

- $\quad$ Gliederung nach der Art der Zurechnung: Dadurch werden Einzelkosten und Gemeinkosten unterschieden:

- Einzelkosten sind den betrieblichen Leistungen direkt zuzuordnen. Sie werden ohne weitere Verrechnung über die Kostenstellen auf die Kostenträger umgelegt z. B. Implantate, Blutersatzstoffe oder Herzschrittmacher, der einem Patienten eingesetzt wird. Die Einzelkosten genügen in hohem Maße dem Verursachungsprinzip ${ }^{227}$

\footnotetext{
226 Quelle: Bopp, M., Hohenbild, R. (1984), S. 69.

227 Vgl. Hentze, J. (1979): Kosten- und Leistungsrechnung in Krankenhäuser systematische Einführung, Köln, Stuttgart, Berlin, Mainz, 1979, S. 51.
} 
- Gemeinkosten sind alle Kosten, die einem Kostenträger nicht direkt zugeordnet werden können. Sie sind für mehrere oder alle Leistungen der Kostenbereiche des Krankenhauses entstanden. Hier zu zählen insbesondere Verwaltungskosten. Bei den Gesamtkosten ist eine Verteilung nach dem Verursachungsprinzip selten oder gar nicht durchzuführen. Daher werden sie indirekt in Form von Zuschlägen mit Hilfe besonderer Schlüssel in der Regel über die einzelnen Kostenstellen auf die Leistungen verrechnet.

- Wenn die Gemeinkosten direkt aus Vereinfachungsgründen zu dem einzelnen Kostenträger zugerechnet werden, so spricht man von unechten Gemeinkosten (z. B. Verbandmittel, die theoretisch den Patienten genau zugerechnet werden könnten), und von echten Gemeinkosten, wenn sie nur indirekt zugerechnet werden können. ${ }^{228}$

- $\quad$ Gliederung nach der Art der Abhängigkeit von der Leistungsmenge: Durch Gliederung der Kosten nach ihrem Verhalten bei Beschäftigungsänderungen, also einer Änderung der Kapazitätsauslastung, lassen sich fixe Kostenarten und variable Kostenarten unterscheiden. Im Krankenhaus wird die Kapazitätsauslastung durch den Grad der Bettenauslastung ausgedrückt, die Zahl der Patienten, die Anzahl der Laborleistungen zur Angabe der Beschäftigung im Laborbereich und die Zahl der Geburten zur Messung der Auslastung des Kreißsaales betrachtet.

- $\quad$ Fixe Kosten sind diejenigen, die konstant bleiben, obwohl die Beschäftigung sich ändert. Sie fallen unabhängig von der Beschäftigung immer in gleicher Höhe an, z. B. Verrechnungen und bestimmte Steuern, Instandhaltung und Geräteüberwachung. Es gibt solche Kosten, die bis zu einer bestimmten Erhöhung des Beschäftigungsgrades konstant bleiben die sich aber durch eine weitere Erhöhung steigern, z. B. Personalkosten für festangestellter Mitarbeiterin der Verwaltung. Solche Kosten werden als sprungfixe Kosten bezeichnet.

- Variable Kosten sind beschäftigungsabhängig. Sie verändern sich proportional, überproportional (progressiv) oder unterproportional (degressiv) mit der

$\overline{228}$ Vgl. Scherrer, G. (1999), S. 24-26. 
Beschäftigungsveränderung, z. B. Arznei-, Heil- und Hilfsmittel, Lebensmittel für Patienten und für Laboruntersuchungen. ${ }^{229}$

Im Allgemeinen sind Einzelkosten variable Kosten. Gemeinkosten können sowohl variable als auch fixe sein, wobei fixe Kosten als Kosten der Betriebsbereitschaft immer Gemeinkosten sind. Tabelle 4 stellt verschiedene Kostenarten und ihren Anteil an fixen und variablen Kosten an den Gesamtkosten eines Krankenhauses dar.

\begin{tabular}{||l|l|l|}
\hline \hline Kostenarten & $\begin{array}{l}\text { Periodenfixe } \\
\text { Kosten } \\
\text { Anteil \% }\end{array}$ & $\begin{array}{l}\text { Periodenvariable } \\
\text { Kosten } \\
\text { Anteil \% }\end{array}$ \\
\hline Personalkosten & 98 & 2 \\
Lebensmittel für Patienten & - & 100 \\
Medizinischer Bedarf & 5 & 95 \\
Wasser, Energie, Brennstoffe & 95 & 5 \\
Wirtschaftsbedarf & 80 & 20 \\
Verwaltungsbedarf & 95 & 5 \\
Steuern, Abgaben, Versicherungen & 100 & - \\
Wirtschaftsgüter & 100 & - \\
Instandhaltung & 100 & - \\
Sonstige Kosten & 100 & - \\
\hline
\end{tabular}

Tab. 4: Anteil des variablen und fixen Anteils bei ausgewählten Kostenarten ${ }^{230}$

- Gliederung nach der Herkunft der Kostengüter: Nach diesem Kriterium werden primäre Kosten und sekundäre Kosten unterschieden:

- Die primären Kostenarten (einfachen, ursprünglich) fallen durch den Verbrauch von Leistungen an, die von außen bezogen werden. Sie entstehen durch den Bezug von Kostengütern am Beschaffungsmarkt (Sachgüter, Arbeitsleistung).

- $\quad$ Sekundäre (zusammengesetzte, gemischte) Kostenarten sind das wertmäßige Äquivalent für die vom Betrieb verbrauchten selbsterstellten innerbetrieblichen Leistungen z. B. Kosten der Wäschereinigung, Instandhaltung und Instand-

229 Vgl. Kloock, J., Sieben, G., Schildbach, T. (1999): Kosten- und Leistungsrechnung, 8., aktualisierte und erw. Aufl., Düsseldorf, 1999, S. 44-49.

230 Quelle: Engelke, D.R., Riefenstahl, R. (1985): Die Auswirkung der Flexibilisierung des Krankenhausbudgets auf die Erlössituation und das Betriebsergebnis des Krankenhauses, in: Krankenhaus- Unschau, 5/1985, S. 334. 
setzung durch eigenes Personal. Sekundäre Kostenarten werden ausschließlich kostenstellenbezogen im Rahmen des Betriebsabrechnungsbogens erfasst.

\subsubsection{Erfassung der Kostenarten}

Um sämtliche im Leistungsprozess angefallenen Kosten in Rahmen der Kostenartenrechnung vollständig sogar eindeutig zu erfassen (Kriterium der Vollständigkeit und Kriterium der Eindeutigkeit), müsste die Zuordnung der Kosten zu den verschiedenen Kostenarten mit Hilfe von Kontierungsvorschriften einheitlich erfolgen (Kriterium der Einheitlichkeit). Die Kontierungsvorschriften sollen in Form eines Kontierungskataloges dokumentiert sein. Darüber hinaus sollte das im Sinne der Wirtschaftlichkeitsbeurteilung eines Betriebs (Zeit- und zwischenbetrieblicher Vergleich) nicht nur während einer Abrechnungsperiode sondern auch im Verhältnis der Abrechnungsperiode untereinander geschehen werden. ${ }^{231}$

Für ein mittelgroßes Krankenhauses könnten sich diejenigen Kostenarten ergeben, die beispielsweise in Abb.16 dargestellt sind.

\begin{tabular}{|c|c|c|}
\hline $\begin{array}{l}\text { Aufwandgleiche Kostenarten } \\
\text { Löhne und Gehälter } \\
\text { Ärztlicher Dienst } \\
\text { Pflegedienst } \\
\text { Medizinisch- technischer } \\
\text { Dienst } \\
\text { Funktionsdienst } \\
\text { Klinisches Hauspersonal } \\
\text { Wirtschafts-, Versorgungs-, } \\
\text { und medizinischer Dienst } \\
\text { Instandhaltungs- und } \\
\text { Instandsetzungsdienst } \\
\text { Verwaltungsdienst } \\
\text { Sonderdienste } \\
\text { Gesetzliche Sozialabgaben } \\
\text { Aufwendungen für } \\
\text { Altersversorgung } \\
\text { Aufwendungen für Beihilfen } \\
\text { und Unterstützungen } \\
\text { Sonstige Personal aufwend- } \\
\text { ungen } \\
\text { Lebensmittel } \\
\text { Medizinischer Bedarf } \\
\text { Arzneien, Heile- u. } \\
\text { Hilfsmittel } \\
\text { Ärztlich verordnete }\end{array}$ & $\begin{array}{l}\text { 10. Verwaltungsbedarf } \\
\text { 11. Aufwendungen für zentrale } \\
\text { Dienstleistungen } \\
\text { 12. Verwaltungsbedarf } \\
\text { 13. Aufwendungen für zentrale } \\
\text { Dienstleistungen } \\
\text { 14. Wirtschaftsgüter mit einer } \\
\text { Nutzungsdauer bis zu } 3 \text { J. } \\
\text { - ..für medizinischen Bedarf } \\
\text { - .. für Wirtschaftsbedarf } \\
\text { - .. für Verwaltungsbedarf } \\
\text { 15. Instandhaltung, } \\
\text { Instandsetzung, Material für } \\
\text { aktivierte Eigenleistungen } \\
\text { 16. Steuern, Abgaben, } \\
\text { Versicherungen } \\
\text { 17. Sonstige Aufwendungen } \\
\text { - Fort- und Weiterbildungen } \\
\text { - Ausbildungsstätten } \\
\text { - Sonstige } \\
\text { Kalkulatorische Kostenarten } \\
\text { 18. Kalkul. Abschreibungen } \\
\text { 19. Kalkul. Zinsen auf das } \\
\text { betriebsnotwendige Kapital } \\
\text { 20. Kalkul. Wagnisse } \\
\text { - Leistungserstellung }\end{array}$ & $\begin{array}{l}\text { - med. Gas } \\
\text { - Strom } \\
\text { - Notstromaggregat } \\
\text { - Lufttechnik } \\
\text { - Sauerstoff } \\
\text { - Hol- und Bringdienst } \\
\text { - Patientenbibliothek } \\
\text { - Arztbibliothek Ko der } \\
\text { 22. Verrechnete Kosten der } \\
\text { Hilfskostenstellen den } \\
\text { Versorgungsbereiches } \\
\text { - Lebensmittellager } \\
\text { - Küche } \\
\text { - Textillager } \\
\text { - Wäscherei } \\
\text { - Wäscherei, } \\
\text { - Fremdleistung } \\
\text { - Näherei } \\
\text { - Bettenzentrale, Dienst- } \\
\quad \text { funktion } \\
\text { - Arzneimittellager } \\
\text { - Allgemeine } \\
\text { Kostenstellen } \\
\text { - Apotheke } \\
\text { - Materiallager } \\
\text { Allgemein }\end{array}$ \\
\hline
\end{tabular}

${ }^{231}$ Vgl. Coenenberg, A. (1992): Kostenrechnung und Kostenanalyse, Landsberg, Lech, 1992, S. 6768. 


\begin{tabular}{|c|c|c|}
\hline 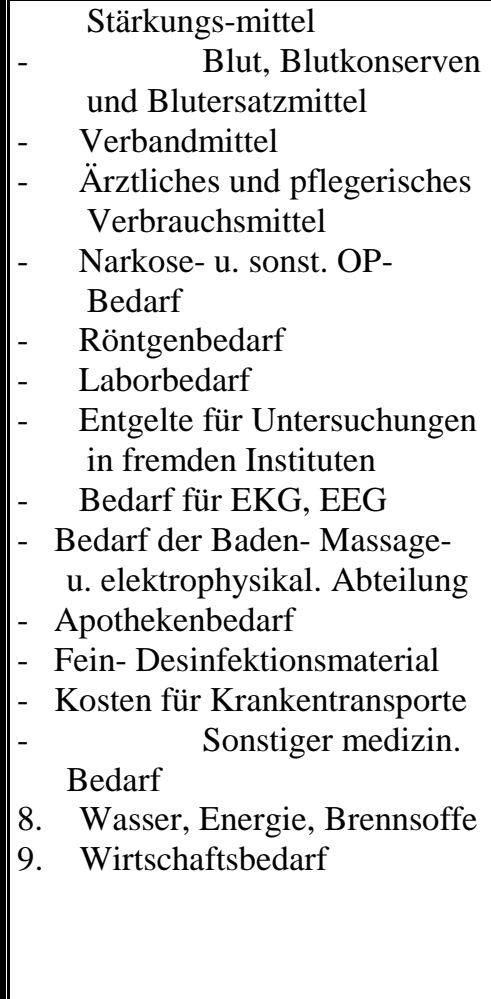 & $\begin{array}{l}\text { swagnis } \\
\text { - } \quad \text { Beständewagnis } \\
\text { - Vertriebswagnis } \\
\text { Sekundäre Kostenarten } \\
\text { 21. Verrechnete Kosten } \\
\text { allgemeiner } \\
\text { Hilfskostenstellen } \\
\text { - Betriebsgebäude } \\
\text { - Arzt- Kasino } \\
\text { - Speisesaal, Teeküche } \\
\text { - Krankenhausleitung } \\
\text { - Personalverwaltung } \\
\text { - Patientenaufnahme, } \\
\text { Leistungsaufnahme, Kasse } \\
\text { - Finanzbuchhaltung } \\
\text { - Posteingang, -ausgang } \\
\text { - Archiv } \\
\text { - Telefonzentrale } \\
\text { - Wirtschaftsleitung } \\
\text { - Werkstätten } \\
\text { - Hausgeistlicher } \\
\text { - Reinigung } \\
\text { - Reinigung Fremdleistung } \\
\text { - Gasheizung }\end{array}$ & $\begin{array}{l}\text { Kostenstellen } \\
\text { - } \text { Lager } \\
\text { 23. Verrechnete Kosten der } \\
\text { Hilfskostenstellen des } \\
\text { Pflege- und } \\
\text { Behandlungsbereiches } \\
\text { - Röntgen } \\
\text { - EKG } \\
\text { - Endoskopie } \\
\text { - Labor } \\
\text { - Bakteriologisches } \\
\text { - Labor } \\
\text { - Bäder, Physikalische } \\
\text { - Therapie } \\
\text { - Anästhesie } \\
\text { - OP- Chirurgie } \\
\text { - OP- Gynäkologie } \\
\text { - OP- Urologie } \\
\text { - OP- HNO } \\
\text { - } \text { Oreißsaal Augen } \\
\text { - Ambulanzchirurgie } \\
\text { - Ambulanzgynäkologie } \\
\text { - Ambulanzurologie } \\
\text { - Ambulanz- Innere }\end{array}$ \\
\hline
\end{tabular}

Abb. 16: Kostenartenplan für ein mittelgroßes Krankenhaus ${ }^{23}$

Grundsätzlich ergibt sich die Erfassung der Kostenarten in einem Krankenhaus aus seiner spezifischen Zielsetzung, nämlich einerseits sind Informationen zur Entscheidungsvorbereitung und zur Kontrolle bereitzustellen, ${ }^{233}$ andererseits muss durch die Übernahme der Daten aus der Finanzbuchhaltung bereits im System der Finanzbuchhaltung gewährleistet sein, dass die Informationen dem Anspruch der Kostenrechnung genügen oder zumindest durch Umrechnungen und Verdichtungen diesen angepasst werden können, da bei isolierter Erfassung ein erheblicher Mehraufwand entsteht. ${ }^{234}$

Im Einzelnen kann die Erfassung der Kosten differenziert oder undifferenziert vorgenommen werden.

- Differenzierte Werterfassung (getrennte Erfassung der Mengen- und Preiskomponente): Hierbei sollte beispielhaft die Ermittlung der Materialkosten herangezogen werden. Im ersten Schritt werden die Verbrauchsmengen erfasst, dann sind die Mengen mit den entsprechenden Preisen (Anschaffungs-, Tages- oder

\footnotetext{
${ }^{232}$ Vgl. Leonhardt, J. (1988), S. 87.

233 Vgl. Leonhardt, J. (1988), S. 81.

${ }^{234}$ Vgl. Dietz, O., Bofinger, W. (1995): Krankenhausfinanzierungsgesetz, Bundespflegesatzverordnung und Folgerecht- Kommentare, Wiesbaden, 1995, S. 76.
} 
Verrechnungspreise) $\mathrm{zu}$ bewerten. Die Bewertung erfolgt nach den in der Finanzbuchhaltung zu befolgenden Vorschriften (handels- und steuerrechtliche Vorschriften). Aufgrund ihrer materiellen Bedeutung ist für die mit der Kostenund Leistungsrechnung $\mathrm{zu}$ erreichenden Ziele der Betriebssteuerung und Wirtschaftlichkeitskontrolle im Krankenhaus die getrennte Mengen- und Preiserfassung auch bei den Personalkosten unverzichtbar. Durch die Gegenüberstellung von Leistungen und Personaleinsatz lassen sich Produktionskennziffern bilden, die als betten- und fallbezogene Belastungsziffern im Zeitvergleich und zwischenbetrieblichen Vergleich Verwendung finden. ${ }^{235}$

- Undifferenzierte Werterfassung: wenn die getrennte Erfassung der Menge und Preiskomponente nicht möglich ist, erfolgt die Erfassung über einen Kostenbetrag z. B. die Kosten der Versicherungen und Gebühren.

\subsubsection{Kostenstellenrechnung}

Die Kostenartenrechnung erfüllt an sich keine eigenständigen Aufgaben, sondern ist sie als eine zielgerechtete Vorbereitungsarbeit für folgende Teilgebiete der Kosten- und Leistungsrechnung zu betrachten. Die Kostenstellenrechnung ist der nächste Schritt der Kostenrechnung, wobei die erfassten und nach Arten gegliederten Kosten auf die Betriebsbereiche verteilt werden sollen. ${ }^{236}$

\subsubsection{Definition und Aufgaben der Kostenstellenrechnung}

Die Kostenstellenrechnung baut auf der Kostenartenrechnung auf, indem nach der Erfassung der Kostenarten sich ihre Verteilung auf die Betriebsbereiche, in denen sie angefallen sind, anschließt. Die Kostenstellenrechnung beantwortet die Fragestellung, wo welche Kosten in welcher Höhe angefallen sind. Die Kostenstellenrechnung wird von den Grundsätzen der Kostenverursachung und der Kosteneinwirkung beherrscht, d. h. die Kostenarten werden am Ort ihrer Entstehung erfasst. Der Ort der Kostenentstehung wird als Kostenstelle bezeichnet. $^{237}$

235 Die Personalrechnung im Krankenhaus umfasst die Personalstatistik als Mengenrechnung und die Lohn- und Gehaltsabrechnung als Wertrechnung. Vgl. Hentze, J., Kehres, E. (1995), S. 48-49.

${ }^{236}$ Vgl. Haberstock, L. (1998), S. 104.

237 Vgl. Kloock, J., Sieben, G., Schildbach, T. (1999), S. 109. 
In besonders einfachen Fällen, wie bei der Einproduktfertigung, lassen sich sämtliche Kosten direkt auf den Kostenträger (das Produkt) zurechnen. Beim Krankenhaus als Dienstleistungs-Mehrproduktunternehmung hingegen lassen sich nur ganz bestimmte Kostenarten (die sog. Kostenträgereinzelkosten) direkt auf die Kostenträger zurechnen (z. B. Medikamente auf die einzelnen Leistungen der Therapie oder auf einen bestimmten Patienten).

Viele Kostenarten (die sog. Kostenträgergemeinkosten) sind dagegen den Kostenträgern nicht direkt zurechenbar (z. B. zeitabhängige Abschreibungen, Steuern auf einzelnen Leistungen oder auf einen bestimmten Patienten). Diese werden zunächst auf die Kostenstellen als den Orten der Kosten- und Leistungsentstehung teils direkt, teils indirekt zugerechnet. Die Kostenstellen verrechnen ihre Kosten dann proportional zu der Inanspruchnahme ihrer Leistungen auf andere Kostenstellen (innerbetriebliche Leistungsverrechnung und Kostenträger). ${ }^{238}$

Die Kostenstellenrechnung bildet den Schwerpunkt der Kostenrechnung, indem ihr im einzelnen folgende Aufgaben zukommen: ${ }^{239}$

- Kontrolle der Wirtschaftlichkeit der einzelnen Kostenstellen: Die Kontenkontrolle der Wirtschaftlichkeit kann an den Stellen durchgeführt werden, an denen die Kosten und Leistungen entstanden sind. Diese Wirtschaftlichkeitskontrolle ist effektiver als die Betrachtung der Wirtschaftlichkeit eines gesamten Betriebs. Eine wichtige Voraussetzung für jede Kostenstelle ist die klare Abgrenzung von anderen Kostenstellen und die Ernennung eines Verantwortlichen, der für die Wirtschaftlichkeit seiner Kostenstelle sensibilisiert ist und dazu Rede und Antwort zu stehen hat. Die Kontrolle der einzelnen Kostenstellen erfolgt durch Soll-Ist-Vergleich, wobei Vergleich der kostenstellenbezogenen Darstellung des prospektiven Budgets mit den tatsächlichen Kosten durchgeführt wird. Das bedeutet auch die Forderung nach einer Plankostenrechnung. Die Wirtschaftlichkeitskontrolle einzelner Kostenstellen erfolgt in Rahmen der Teilkostenrechnung lediglich auf Basis der variablen Kosten, da der Kostenstellenverantwortliche nur für die Kosten verantwortlich gemacht werden

${ }^{238}$ Vgl. Bopp, M., Hohenbild, R. (1984), S. 107. 
kann, die er durch eine Variation der Menge beeinflussen kann. Innerhalb die Grenzplankostenrechnung werden damit nur die variablen Kosten als Plan- bzw. Sollkosten je Kostenstelle ermittelt. Die fixen Kosten sind als Periodenkosten auszuweisen und somit nicht auf einzelne Leistungseinheiten im Sinne des Verursachungsprinzips zu verrechnen. ${ }^{240}$

- Planung und Überwachung der Kostenstellenbudgets: Die Kostenstellenverantwortlichen (im Krankenhaus sind sie in der Regel die Chefärzte), können über die relevanten Kosten- und Leistungsdaten informiert werden. Das besetzt eine hohe Priorität bei der Planung und internen Budgetierung und somit auch bei der Kostenkontrolle voraus. Die Transparenz des Betriebes von einzelnen Kostenstellen über Kostenstellengruppen bis hin zum Gesamtbetrieb ist Primärziel der Kostenstellenrechnung. ${ }^{241}$ Die Beeinflussbarkeit der Kosten im Krankenhaus bezieht sich auf das Vermeiden von Kosten der Kostenstellen, z. B. von nicht zwingend notwendigen Mehrfach-Untersuchungen, des Verfalls von Arzneimittel und fehlender Abstimmung von Urlaubsplanung usw., damit wird eine Budgetvorgabe zur Lenkung ermöglicht.

- Vorbereitung der Kostenträgerrechnung: Die unterschiedliche Beanspruchung einer Kostenstelle durch die verschiedenen Leistungsarten verursacht einen unterschiedlichen Kostenanteil. Dieser kann erst durch die Kostenstellenrechnung in der Kostenträgerrechnung richtig zugeordnet werden. Damit wird die Genauigkeit der Kostenträgerzeitrechnung und Kostenträgerstückrechnung erhöht.

\subsubsection{Bildung und Einteilung von Kostenstellen}

Um die Aufgaben der Kostenstellenrechnung erfüllen zu können, müssen einige Grundsätze beachten werden.

\footnotetext{
${ }^{239}$ Vgl. Schweitzer, M., Küpper, H. U. (1995): System der Kosten- und Erlösrechnung, 6. Aufl., München, 1995, S. 126; auch Vgl. Koch, J. (1998): Gesundheitsökonomie, Betriebswirtschaftliche Kosten- und Leistungsrechnung, München, Wien, Oldenburg, 1998, S. 65-66.

${ }^{240}$ Vgl. Hummel, S., Männel, W.(1983) S. 140.

241 Vgl. Rippel, W. H., Posininsky, R. (1990): Praktische Anwendung der Kostenarten- und Kostenstellenrechnung, in: das Krankenhaus, 11/ 1990, S. 486.
} 
- $\quad$ Bei der Bildung von Kostenstellen ist darauf Wert zu legen, dass ihre Verantwortlichen in der Lage sein sollten. Kostenstellen sollen ein gegenüber anderen abgegrenzter eigener Verantwortungsbereich sein, wodurch eine effektive Wirtschaftlichkeitskontrolle ermöglicht wird. Für jede Kostenstelle muss ein Leiter verantwortlich sein, der die Kosten zu vertreten hat. ${ }^{242}$

Deutliche Bezugsgrößen für die anfallenden Kosten und die erstellten Leistungen müssen für jede Kostenstelle gefunden werden. Dies ist ein Ausgangspunkt für die Kostenkontrolle und die Kalkulation. Die Bestimmung richtiger Bezugsgrößen ist um so einfacher, je aufrecht die Kostenstellengliederung durchgeführt wird. ${ }^{243}$

- Die Kostenstellengliederung sollte aber nur soweit gebildet werden, wie sie nach dem Wirtschaftlichkeitsprinzip vertreten werden kann.

Im allgemein können die Kostenstellen nach verschiedenen Kriterien, die miteinander kombiniert sind, gebildet werden:

- Funktionale Abgrenzung,

- Verantwortungsbereiche,

- Räumliche Abgrenzung und

- Abrechnungstechnische Verfahren.

In der Tat kann eins oder mehrere diesen Kriterien auf die Bildung der Kostenstellen wirken. Abb. 17 stellt die Bestimmungsfaktoren für die Bildung der Verantwortungsbereiche (Kostenstellen) dar.

${ }^{242}$ Vgl. Trill, R. (2000), S. 416. 


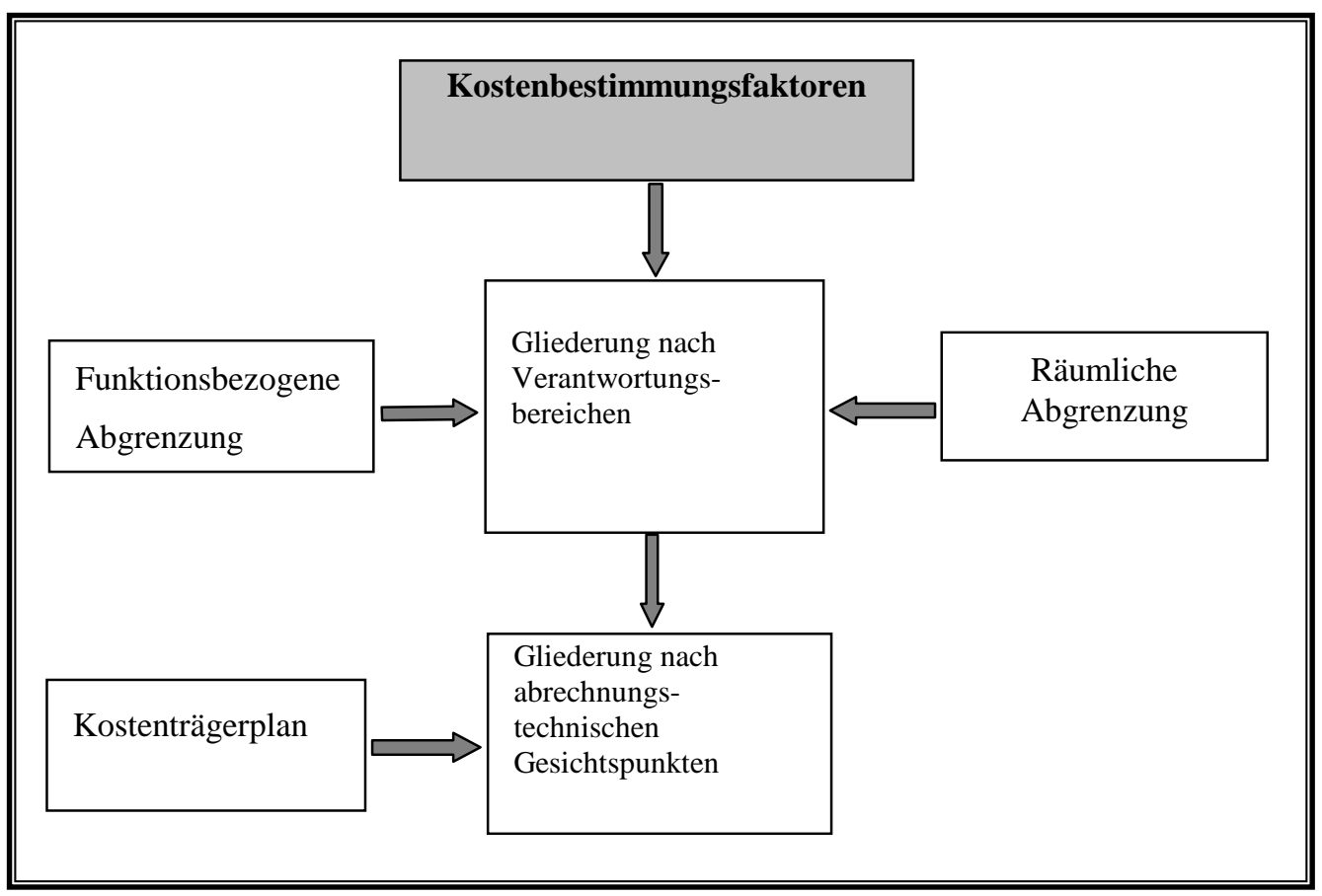

Abb. 17: Verantwortungsbereiche und Kostenbestimmungsfaktoren. ${ }^{244}$

Die Funktionsbereiche sind ein Kriterium, nach dem die Kostenstellen in Betrieben der Wirtschaft untergliedert sind. Das gilt auch im Krankenhausbetrieb. So wird ein Krankenhaus in Kostenstellen wie z. B. Versorgungseinrichtung, medizinische Bereiche, Pflegebereiche, Verwaltungsstellen, Forschungsstellen und sonstige Kostenstellen eingeteilt. Diese Kostenstellen sollen gleichzeitig einzelne Verantwortungsbereiche darstellen, da Kostenstellenverantwortung bzw. Ergebnisverantwortung die Voraussetzung für Kostenkontrolle, aber auch für Kostenplanung und für moderne Managementmethoden ist. Die Einteilung nach Funktionsbereichen mit der Bildung der Kostenstellen nach Verantwortungsbereichen ist deckungsgleich, sofern der Organisationsplan für die Funktionsbereiche die Kompetenzen klar abgrenzt. Diese Gliederung hat den Vorteil, dass der jeweilige Kostenstellenleiter zur Verantwortung gezogen werden kann. $^{245}$

\footnotetext{
${ }^{243}$ Vgl. Hentze, J. (1979), S. 87.

244 Quelle: Leonhardt, J. (1988), S. 89.

245 Kloock, J., Sieben, G., Schildbach, T. (1999): Kosten- und Leistungsrechnung, 8., aktualisierte und erw. Aufl., Düsseldorf, 1999, S. 111.
} 
Räumliche Gliederungskriterien dominieren, sofern eine Kostenstelle einen räumlich zusammenhängenden und abgegrenzten Bereich darstellt. Die Einteilung nach räumlichen Gesichtspunkten ist aber für die Kostenstellenrechnung nur dann zweckmäßig, wenn gleichzeitig eine Gliederung nach Verantwortungsbereichen durchgeführt wird, da andernfalls mehrere Kostenstellenleiter für die Kostenstelle verantwortlich wären. Ist die Einteilung nach Verantwortungsbereichen Hauptkriterium, so können anderen Abgrenzungen wie funktionsbezogene und räumliche Kriterien als Subkriterien bei der Kostenstellenbildung einfließen..

Im Krankenhaus könnten bedeutende Bestimmungsgründe für die Tiefe der Kostenstellengliederung wie die Größe des Krankenhauses, das Leistungsprogramm, die Ablauf- und Aufbauorganisation des Krankenhauses und die verfolgten Kostenrechnungszwecke sein. Als Beispiel ist unter dem Sichtpunkt Kostenstellenverantwortlichkeit eine detaillierte Differenzierung für die Kostenstelle „Küche“ in Hauptküche, Diätküche, Personalküche usw. vorzunehmen. Daraus ergibt sich eine differenziertere Leistungserfassung, die wiederum eine exakte Analyse der Kosten der Kostenstellen ermöglichen. ${ }^{246}$ In Tab. 5 sind die Verantwortungsbereiche eines Krankenhauses dargestellt. Dabei sind auch räumliche und funktionale Kriterien berücksichtigt.

\begin{tabular}{|c|c|c|c|}
\hline $\begin{array}{l}\text { Dezentrale } \\
\text { Bereiche }\end{array}$ & \multicolumn{3}{|c|}{ Zentrale Bereiche } \\
\hline $\begin{array}{l}\text { I. Dezentraler } \\
\text { medizinischer } \\
\text { Bereich }\end{array}$ & $\begin{array}{l}\text { II. Zentraler } \\
\text { medizinischer } \\
\text { Bereich }\end{array}$ & \begin{tabular}{|l} 
III. \\
Versorgungsbereich
\end{tabular} & $\begin{array}{l}\text { IV. } \\
\text { Verwaltungsbereich }\end{array}$ \\
\hline $\begin{array}{l}\text { Innere Medizin } \\
\text { Chirurgie } \\
\text { Neurochirurgie } \\
\text { Umfallchirurgie } \\
\text { Urologie } \\
\text { Kieferchirurgie } \\
\text { Orthopädie } \\
\text { Gynäkologie } \\
\text { Geburtshilfe } \\
\text { Pädiatrie } \\
\text { HNO } \\
\text { ZMK }\end{array}$ & $\begin{array}{l}\text { Laboratoriums- } \\
\text { diagnostik } \\
\text { Blutversorgung } \\
\text { Funktionsdiagnosti } \\
\quad \text { k } \\
\text { Endoskopie } \\
\text { Strahlendiagnostik } \\
\text { Nuklearmedizin } \\
\text { Strahlentherapie } \\
\text { Operationsbereich } \\
\text { Anästhesie } \\
\text { Intensivmedizin }\end{array}$ & $\begin{array}{l}\text { a. medizinischer } \\
\text { Versorgungsberei } \\
\text { ch } \\
\text { Apotheke mit } \\
\text { Verbandmittelaus } \\
\text { gabe } \\
\text { Zentralstrlisation } \\
\\
\text { b. Wirtschaftlich- } \\
\text { technischer } \\
\text { Bereich } \\
\text { Küche }\end{array}$ & $\begin{array}{l}\text { Hauptabteilung } \\
\text { Patientenabteilung } \\
\text { Betriebswirtschaftli } \\
\text { che- } \\
\text { Abteilung } \\
\text { wirtschaftsführungs } \\
\text { - } \\
\text { abteilung } \\
\text { Technische } \\
\text { Abteilung }\end{array}$ \\
\hline
\end{tabular}

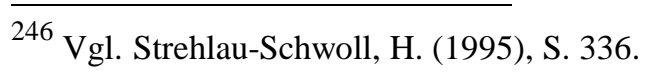




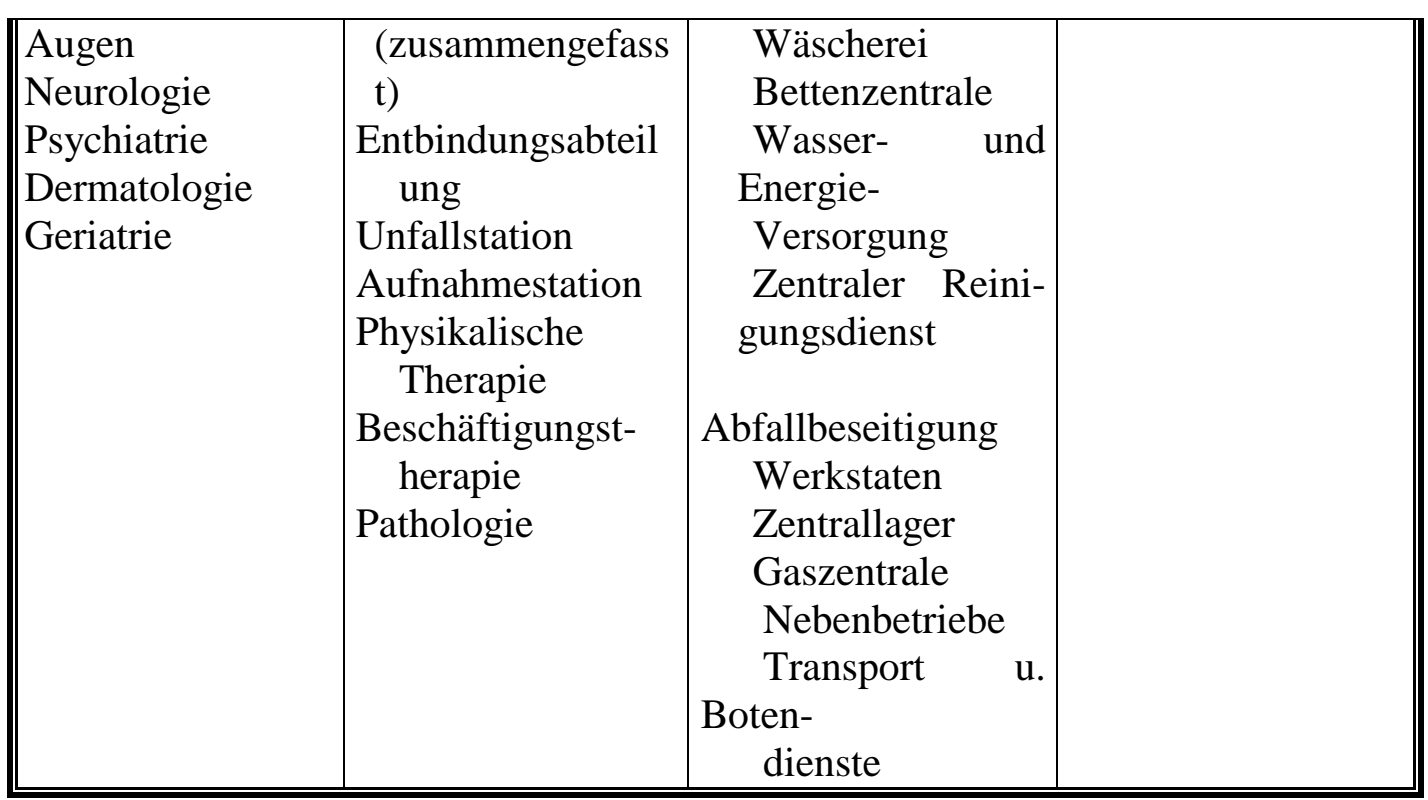

Tab. 5: Verantwortungsbereiche des Krankenhauses. ${ }^{247}$

Nach Abrechnungsverfahren-Kriterien kann man Hauptkostenstellen, Hilfskostenstellen $^{248}$ und Nebenkostenstellen unterscheiden. In den Hauptkostenstellen werden eigentlichen Leistungen eines Krankenhauses erstellt. Die Hilfskostenstellen dienen mittelbar zur Leistungserstellung. Sie geben innerbetriebliche Leistungen an andere Kostenstellen weiter. Bei den Hilfskostenstellen können allgemeine Hilfskostenstellen, Hilfskostenstellen im Behandlungsbereich und im Versorgungsbereich unterschieden werden. In den Nebenkostenstellen werden Leistungen erbracht, die nicht zum eigentlichen Leistungsprogramm des Krankenhauses gehören. ${ }^{249}$

Auch unter rechnungstechnischen Gesichtpunkten kann man Vor- und Endkostenstellen unterscheiden. Die Kosten der Vorkostenstellen werden im Rahmen der Kostenstellenrechnung auf die ihre Leistungen empfangenden Endkostenstellen weiter umgelegt. Von den Endkostenstellen werden die Kosten direkt auf den Kostenträger verrechnet. In Tab.. 6 wird die Bildung der Kostenstellen eines mittleren Krankenhauses nach dem Rechnungsverfahren dargestellt.

\footnotetext{
247 Quelle: Leonhardt, J. (1988), S. 90.

248 Auch werden Hauptkostenstellen als primäre Kostenstellen und die Hilfskostenstellen als sekundäre Kostenstellen bezeichnet.

${ }^{249}$ Vgl. Hentze, J. (1979), S. 88.
} 


\begin{tabular}{|c|c|c|c|c|}
\hline \multicolumn{3}{|c|}{ Hilfskostenstellen } & \multirow{2}{*}{$\begin{array}{c}\text { Haupt- } \\
\text { kostenstellen }\end{array}$} & \multirow{2}{*}{$\begin{array}{c}\text { Neben- } \\
\text { kostenstellen }\end{array}$} \\
\hline Allgemeine & $\begin{array}{c}\text { Versorgungs- } \\
\text { bereich }\end{array}$ & $\begin{array}{l}\text { Pflege- und } \\
\text { Behandlungs- } \\
\text { bereich }\end{array}$ & & \\
\hline $\begin{array}{l}\text { Betriebsgebäud } \\
\text { Betriebsgebäud } \\
\text { e } 2 \\
\text { Arzt-Kasino } \\
\text { Speisesaal, } \\
\text { Teeküche } \\
\text { Krankenhausleit } \\
\text { ung } \\
\text { Personalverwalt } \\
\text { ung } \\
\text { Patientenaufnah } \\
\text { me/ } \\
\text { Leistungsabrech } \\
\text { nung } \\
\text { Kasse } \\
\text { Finanzbuchhalt } \\
\text { ung } \\
\text { Posteingang/ } \\
\text { ausgang } \\
\text { Archiv } \\
\text { Telefonzentrale } \\
\text { / Pforte } \\
\text { AKH und KKH } \\
\text { Wirtschaftsleitu } \\
\text { ng } \\
\text { Werkstätten } \\
\text { Hausgeistlicher } \\
\text { Reinigung } \\
\text { Reinigung } \\
\text { Fremdleistung } \\
\text { Gasheizung } \\
\text { Med. Gas } \\
\text { Wasser, Strom, } \\
\text { Not- } \\
\text { stromaggregat, } \\
\text { Luft- technik }\end{array}$ & $\begin{array}{l}\text { Lebensmittell } \\
\text { Küche } \\
\text { Textillager } \\
\text { Wäscherei } \\
\text { Wäscherei } \\
\text { Fremdleistung } \\
\text { Näherei } \\
\text { Bettenzentrale } \\
\text { / Desin - } \\
\text { fenktion } \\
\text { Arzneimittella } \\
\text { ger } \\
\text { Allgemeine } \\
\text { Kostenstelle } \\
\text { Apotheke } \\
\text { Materiallager } \\
\text { (Büromaterial } \\
\text { Haushaltsver- } \\
\text { brauchsartikel } \\
\text {, med. Lager) } \\
\text { Allgemeine } \\
\text { Kostenstelle } \\
\text { Lager }\end{array}$ & $\begin{array}{l}\quad \text { Röntgen 1 } \\
\text { Röntgen } 2 \\
\text { EKG 1 } \\
\text { EKG 2 } \\
\text { Endoskopie } \\
\text { Labor 1 } \\
\text { Labor 2 } \\
\text { Bakteriologisch } \\
\text {. Labor } \\
\text { Bäder/ physi. } \\
\text { Therapie } \\
\text { Anästhesie } \\
\text { OP- Chirurgie } \\
\text { OP- } \\
\text { Gynäkologie } \\
\text { OP- Urologie } \\
\text { OP- HNO } \\
\text { OP- Augen } \\
\text { Kreisaal } \\
\text { Ambulanz- } \\
\text { Chirurgie } \\
\text { Ambul.- } \\
\text { Gynäkologie } \\
\text { Ambulanz- } \\
\text { Urologie } \\
\text { Ambulanz- } \\
\text { Innere }\end{array}$ & \begin{tabular}{l}
\multicolumn{1}{c}{ Innere Medizin } \\
Frauenstation \\
Männerstation \\
Infektionsstation \\
Privatstation \\
Pädiatrie un \\
Früh- und \\
Neugeborenen- \\
station \\
Klein- \\
Schulkinderstation \\
Säuglings- und \\
Krabbler- station \\
Infektionsstation \\
Allgemeine \\
Chirurgie \\
Frauenstation \\
Männerstation \\
Privatstation \\
Chirurgie \\
Urologie \\
Orthopädie \\
Gynäkologie \\
Geburtshilfe \\
HNO \\
Augen \\
Infektionsstation
\end{tabular} & $\begin{array}{l}\quad \text { Wohnheim } 1 \\
\text { Wohnheim } 2 \\
\text { Krankenpflegesc } \\
\text { hule 1 } \\
\text { Krankenpflegesc } \\
\text { hule 2 } \\
\text { Röntgen 1 } \\
\text { Röntgen 2 } \\
\text { EKG 1 } \\
\text { EKG 2 } \\
\text { Endoskopie } \\
\text { Labor 1 } \\
\text { Labor 2 } \\
\text { Bakteriologische } \\
\text { s Labor } \\
\text { Ambulanz- } \\
\text { Chirurgie } \\
\text { Ambul.- } \\
\text { Gynäkologie } \\
\text { Ambulanz- } \\
\text { Urologie } \\
\text { Ambulanz-Innere } \\
\text { Bäder/ physi. } \\
\text { Therapie }\end{array}$ \\
\hline
\end{tabular}

Tab. 6: Bildung der Kostenstellen eines Krankenhauses. ${ }^{250}$

$\overline{250}$ Quelle: Leonhardt, J. (1988),S. 93. 


\subsubsection{Durchführung der Kostenstellenrechnung}

Die letzte Stufe der Kostenstellenrechnung ist die Verteilung aller primären Kosten, die im Rahmen der Kostenstellenskontierung den betreffenden Kostenstellen als Kostenstelleneinzelkosten direkt zugeordnet wurden, auf die leistungsempfangenden Kostenstellen. Damit müssen auch alle Kosten der Vorkostenstellen auf die leistungsempfangenden Endkostenstellen übergeleitet werden. Die Verteilung der Kosten kann grundsätzlich auf zweierlei Art und Weise durchgeführt werden auf Grundlage tatsächlich beanspruchter Leistungen (innerbetriebliche Leistungsverrechnung) und auf Grundlage von Verteilungsschlüsseln (Umlagenrechnung).

Verfahren der innerbetrieblichen Leistungsverrechnung: Ein Betrieb erstellt nicht nur die marktorientierten Leistungen, sonder auch Leistungen, die er selbst konsumiert (innerbetriebliche Leistungen) wie z. B. Reparaturleistungen, selbsterstellte Anlagen bzw. Modelle, eigene Transportleistungen oder Erzeugung von Strom. Die Kosten der innerbetrieblichen Leistungsverrechnung sollen entsprechend ihrer Inanspruchnahme auf andere Kostenstellen verteilt werden. Die Verrechnung innerbetrieblicher Leistungen ist für die Zwecke der Kostenkontrolle, Ermittlung der Selbstkosten und dem Wirtschaftlichkeitsvergleich zwischen Eigen- und Fremderstellung erforderlich. $^{251}$

Im Krankenhaus werden Leistungen nicht nur direkt für Patienten, sondern auch Leistungen, die indirekt für ihn erstellt (innerbetriebliche Leistungen) erbracht wie selbsterstellte Dienstleistungen (z. B. Laborleistungen, Röntgenbilder, Reparaturleistungen, Wäschereinigung, Speisen), selbsterstellte Güter, die im Krankenhaus wieder verbraucht werden (z. B. selbsterstellte Medikamente, Strom), und selbsterstellte Güter, die über einen Zeitraum Nutzungen abgeben können (z. B. selbsterstellte Anlagen). Die exakte Erfassung der Kosten eines Krankenhauses ist nur dann möglich, wenn eine Leistungsverrechnung von den Instituten, also den nicht- Kliniken stattfinden. 
Beispielsweise müssen zur Kostenermittlung einer Unfallchirurgie die Kosten der Blutzentrale und die Anästhesie in Ansatz betrachtet werden. ${ }^{252}$

In innerbetrieblichen Leistungen unterscheidet sind folgende Grundtypen der Leistungsverflechtung zu betrachten:

a. Einseitige, einstufige Leistungsabgaben an eine Kostenstelle, wobei alle Kosten einer Vorkostenstelle an eine Endkostenstelle abgegeben werden, z. B. die Vorkostenstelle Herz-OP-Bereich bringt nur für die Endkostenstelle Herzchirurgie.

b. Einseitige, einstufige Leistungsabgaben an mehrere Kostenstelle: die Kosten einer Vorkostenstelle werden an mehrere Endkostenstellen abgegeben, z. B. die Vorkostenstelle Labor erbringt Leistungen für alle bettenführenden Abteilungen.

c. Einseitige, mehrstufige Leistungsabgaben: die Leistungen einer Vorkostenstelle werden in einer anderen Vorkostenstelle verrechnet, die ihre Leistungen wiederum an Endkostenstellen abgibt, z. B. das Labor bringt Untersuchungen für den OP-Bereich, der seine Leistungen an die Endkostenstelle Chirurgie weiterberechnet.

d. Wechselseitige Leistungsabgaben: Hier beliefern sich mindestens zwei Kostenstellen gegenseitig, z. B. Pflegefachbereiche, in denen für Patienten eines anderen Pflegebereichs Konsiliarleistungen erbracht werden. Diese Leistungsverflechtung tritt im Krankenhaus nur sehr selten auf, so dass sie bei der Auswahl der Verrechnungsverfahren meistens unberücksichtigt bleibt. Abb. 18 stellt die Typen innerbetrieblicher Leistungsverflechtungen dar:

${ }^{251}$ Vgl. Haberstock, L., (1998), S. 124-125.

252 Vgl. Schwarz, R. (1993): Modernes Management in öffentlichen Krankenhäusern, wie kann die Krankenhausleitung den Konsequenzen des GSG begegnen, in: das Krankenhaus 3/ 1993, S. 117. 


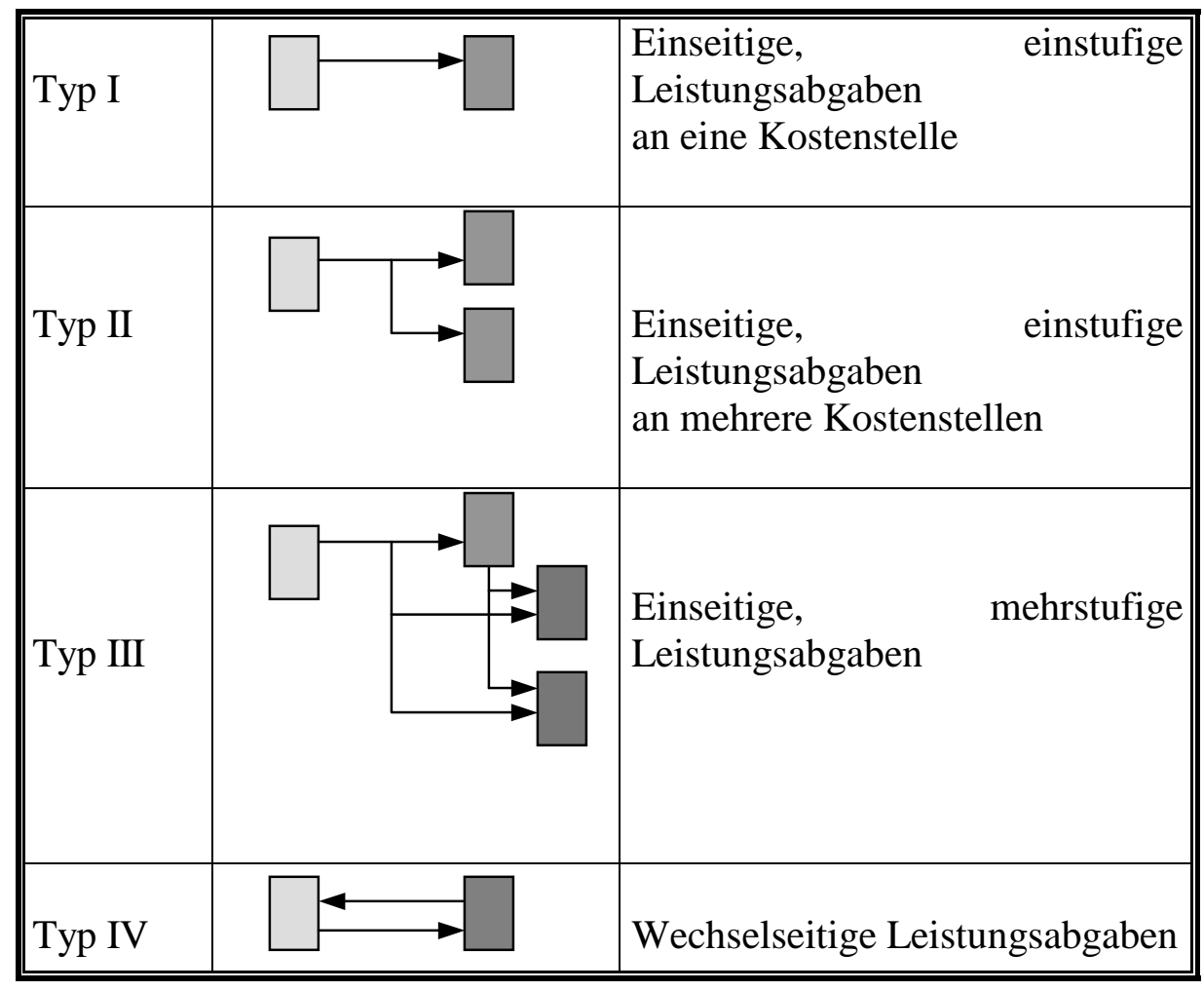

Abb. 18: Typen innerbetrieblicher Leistungsverflechtungen. ${ }^{253}$

Nach den Leistungsverflechtungen, die im Krankenhaus auftreten, muss von den unterschiedlichen Verfahren der innerbetrieblichen Leistungsverrechnung bzw. der Kostenumlage, die in Abb. 19 dargestellten sind, ein geeignetes Verfahren ausgewählt werden.

${ }^{253}$ Quelle: Hentze, J., Kehres, E. (1995), S.94. 


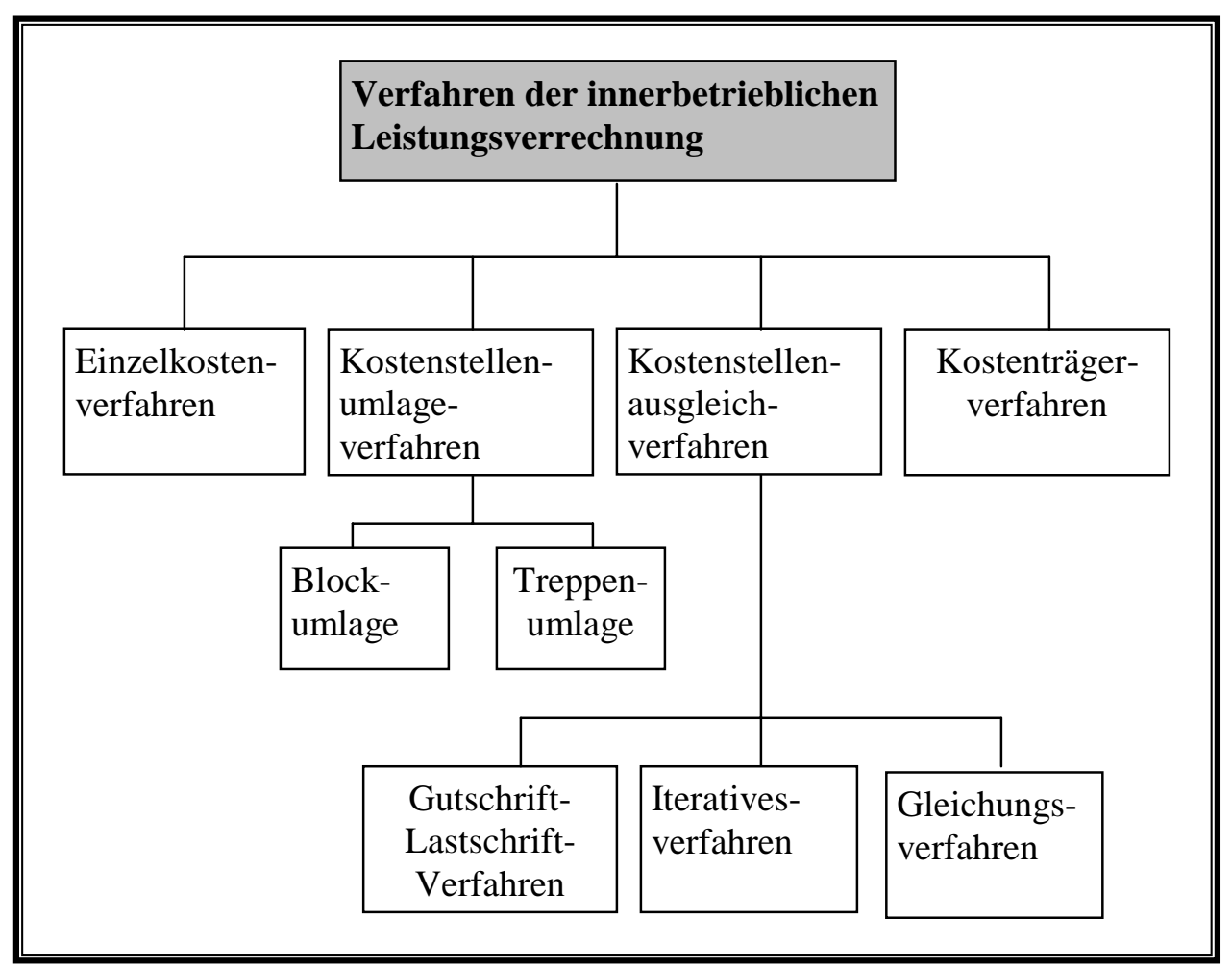

Abb. 19: Verfahren der innerbetrieblichen Leistungsverrechnung. ${ }^{254}$

Das Einzelkostenverfahren (Kostenartenverfahren) findet nur Anwendung, wenn die innerbetrieblichen Leistungen in Haupt- bzw. Endkostenstellen erbracht werden. ${ }^{255}$

Das Kostenstellenumlageverfahren wird bei der mehrstufigen, einseitigen Leistungsverflechtung angewendet, wenn Kostenstellen spezielle innerbetriebliche Leistungen erbringen. Es wird vom Kostenartenverfahren abgewichen, da sowohl die Einzelkosten als auch die anteiligen Gemeinkosten der innerbetrieblichen Leistungen auf eigens hierfür eingerichteten Hilfskostenstellen erfasst werden. ${ }^{256}$

Beim Blockumlageverfahren werden die primären Kosten sämtlicher Vorkostenstellen zu sekundären Kosten der Endkostenstellen verrechnet.

254 Quelle: Schweitzer, M., Küpper, H. U. (1995), S. 140.

255 Vgl. Schoenfeld, H., Möller, H.(1995), S.148.

256 Vgl. ebenda, S. 148-149. 
Das Treppenumlageverfahren verteilt die Kosten der Kostenstellen auf die jeweils nachgelagerten Vor- und Endkostenstellen. Hierbei muss die Reihenfolge der Vorkostenstellen und damit die Reihenfolge der Kostenverteilung so gewählt werden, dass die zuerst abgerechneten Kostenstellen möglichst keine Leistungen von nachgelagerten Kostenstellen erhalten. Das Verfahren wird so lange fortgeführt, bis alle Vorkostenstellen ihre Kosten verteilt haben. $^{257}$

Bei den wechselseitigen Leistungsverfahren müssen die Kosten aller innerbetrieblichen Leistungen simultan gleichzeitig durch mathematisches Verfahren (Gleichungsverfahren) verrechnet werden.

Bei den Kostenträgerverfahren werden die innerbetrieblichen Leistungen wie Leistungen für Dritte als selbständige Kostenträger kalkuliert. Dabei werden die Einzelkosten und die Gemeinkosten der innerbetrieblichen Leistungen mit Hilfe von Kalkulationsverfahren bestimmt.

Das System des Betriebsrechnungsbogens: Der Betriebsrechnungsbogen (BAB) ist traditionell ein organisatorisches Hilfsmittel zur Verrechnung der Kostenträgerkosten auf die Kostenstellen sowie Kostenträger und als Arbeitsanweisung zur Realisierung der Aufgaben der Kostenstellenrechnung zu verstehen. Der BAB erfasst grundsätzlich nur die Ggemeinkosten. Die Einzelkosten werden den Kostenträgern direkt und verursachungsgemäß zugerechnet. Aus Übersichtlichkeits- und Vollständigkeitsgründen können Einzelkosten dennoch im BAB für Zwecke des Informierens aufgenommen werden $^{258}$

Der Betriebsabrechnungsbogen verbindet die Kostenarten- und Kostenträgerrechnung miteinander. Die Kostenträgergemeinkosten werden aus der Kostenartenrechnung in den $\mathrm{BAB}$ übernommen und dann auf diejenigen Kostenstellen verteilt, in denen sie angefallen sind. Danach läßt sich die Summe der primären Kostenträgergemeinkosten für jede Kostenstelle feststellen.

\footnotetext{
${ }^{257}$ Vgl. Freidank, C. (1994). S. 140-141.

258 Vgl. Kloock, J., Sieben, G., Schildbach, T.(1999), S.113-115.
} 
Summiert man diese Kosten über alle Kostenstellen, so muss sich wieder die Summe der aus der Kostenartenrechnung übernommenen Beträge ergeben, denn in der Kostenstellenrechnung wird diese Summe lediglich auf Kostenstellen verteilt. $^{259}$

Im Aufbau des Betriebsabrechnungsboges des Krankenhauses werden alle kostenstellenbezogen erfassten Kostenarten in der vertikalen Auflistung aufgeführt. in der horizontalen Gliederung werden die Kostenstellen aufgeführt. Sie sollen in Endkostenstellen (Normalpflege und abweichende Pflege) und Vorkostenstellen (die gemeinsamen Kostenstellen sowie die Institutionen der Versorgungs- und Medizinischeinrichtungen) gegliedert werden. Darüber hinaus müssen Für die Kostenabrechnung und Kosten- sowie Wirtschaftlichkeitskontrolle auch die Einzelkosten des Krankenhauses im BAB ausgewiesen werden. ${ }^{260}$

\subsubsection{Kostenträgerrechnung}

Nachdem die angefallenen Kosten in der Kostenartenrechnung erfaßt und in der Kostenstellenrechnung auf die Endkostenstellen weiterverrechnet worden sind, werden sie innerhalb der Kostenträgerrechnung auf die Kostenträger verteilt. Somit bildet die Kostenträgerrechnung die letzte Stufe der Kostenrechnung ${ }^{261}$

\subsubsection{Definition und Aufgabe der Kostenträgerrechnung}

Kostenträger sind die betrieblichen Leistungen. Aus Sicht der Kostenrechnung sind Kostenträger die selbständige Leistungseinheit wie z. B. Einzelauftrag, Hergestellte Produkteinheit oder bereitgestellte Dienstleistung, die den Güter- und Leistungsverbrauch ausgelöst haben. Folglich ist die Abgrenzung der Kostenträger unproblematisch, sofern die Leistungseinheiten begreiflicherweise nach Branchenzugehörigkeit definiert werden können. ${ }^{262}$ Allerdings werden häufig zur Erhöhung der Übersichtlichkeit Kostenträger zu Kostenträgergruppen (Leistungsgruppen)

\footnotetext{
${ }^{259}$ Vgl. Bopp, M., Hohenbild, R. (1984), S. 116.

${ }^{260}$ Vgl. Keun, F. (1997), S. 135-136.

261 Vgl. Haberstock, L. (1998), S. 142.

${ }^{262}$ Vgl. Cohnenberg, A., (1992), S. 92.
} 
zusammengefasst, wenn die Zahl der unterschiedlichen Leistungseinheiten sehr groß ist. $^{263}$

Im Krankenhaus sind Kostenträger aus Sicht der Kostenrechnung alle Krankenhausleistungen, durch die Kosten verursacht werden. Dementsprechend müssen die Verursacher der Kosten nach dem Verursachungsprinzip die entstandenen Kosten tragen. ${ }^{264}$ In der Regel wird der Patient als „Leistungseinheit“ bestimmt.

Kostenträger sind sowohl Absatzleistungen (Marktleistungen, Endleistungen) als auch innerbetriebliche Leistungen. Die Absatzleistungen (Hauptkostenträger) entsprechen den ,abrechenbaren Leistungen“, die sich in stationäre und ambulante Leistungen untergliedern, und den innerbetrieblichen Leistungen ${ }^{265}$ (Hilfskostenträger) mit den dazu benötigten Vorleistungen (Abb. 21).

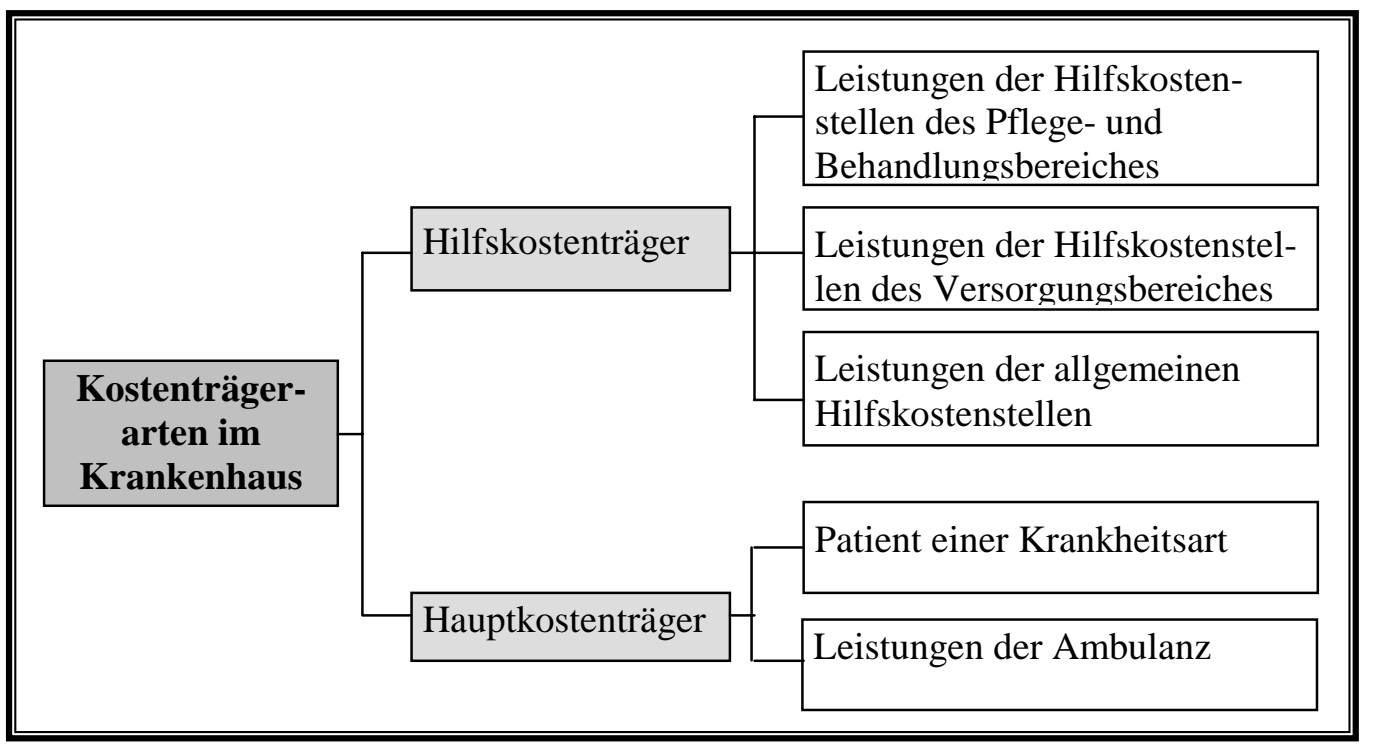

Abb. 21: Kostenträgerarten im Krankenhaus. ${ }^{266}$

Die Aufgaben der Kostenträgerrechnung sind nach der Blickrichtung zwischen periodenbezogener Kostenträgerzeitrechnung (Betriebsergebnisrechnung) oder objektbezogenen Kostenträgerstückrechnung (Selbstkostenrechnung, Kalkulation) zu unterscheiden. Während die Aufgabe der Kostenträgerstückrechnung die Ermittlung der Kosten für eine einzelne Leistungseinheit ist, ist die Aufgabe der

\footnotetext{
263 Vgl. Schoenfeld, H., Möller, H.(1995), S.167.

${ }^{264}$ Vgl. Heinen, E. (1978), S. 107-108.

265 Die Verrechnung innerbetrieblicher Leistungen ist grundsätzlich im Rahmen der Kostenstellenrechnung behandelt worden. Siehe 4.1.2.3.

266 In Anlehnung an Leonhardt, J. (1988), S.129.
} 
Kostenträgerzeitrechnung die Gegenüberstellung aller in einer Abrechnungsperiode entstandenen Kosten und der dafür erzielten Erlöse.

Die Hauptaufgaben der Kostenträgerzeitrechnzng besteht in Durchführung der kurzfristigen Erfolgsrechnung (Betriebsergebnisrechnung), wobei die nach Leistungsarten gegliederten insgesamten Kosten, die in der Abrechnungsperiode angefallen sind, ermittelt werden. Darüber besteht die Hauptaufgaben der Kostenträgerstückrechnung in der Ermittlung der Selbst- bzw. Herstellkosten der betrieblichen Leistungseinheiten oder -Einheitsgruppen, wobei die Unterlagen für preispolitische Entscheidungen sowie als Ausgangsdaten für Planungsaufgaben dienen. $^{267}$

Da die Kostenträgerstückrechnung patientenbezogen ausgestaltet werden sollte, wobei die Kosten möglichst dort erfaßt werden sollen, wo sie anfallen, besitzt sie im Krankenhaus neben der Ermittlung der Kostenträgerkosten folgende Aufgaben:

Ermittlung der Angebotspreise und Preisuntergrenzen: Die Preisbildung gilt als Hauptaufgabe der Kostenträgerrechnung, da bei vielen Entgeltsystemen der Krankenhäuser die Ermittlung von Preisuntergrenzen existentielle Bedeutung gewinnt.

- $\quad$ Kontrolle der Wirtschaftlichkeit: Die Wirtschaftlichkeitskontrolle ist zwar wesentliche Aufgabe der Kostenstellenrechnung, jedoch kann auch die Kostenträgerrechnung die bezüglich Informationen liefern, womit sie durch die Feststellung der Selbstkosten/ Herstellkosten Vergleichsmöglichkeiten mit anderen vergleichbaren Krankenhausbetrieben dient. Andererseits können mit Hilfe der Kostenträgerrechnung auch Vergleiche einzelner, intern erbrachter Teilleistungen (beispielsweise die eigentlichen Kernaktivitäten nur begleitende Dienstleistungen wie Speisenversorgung und Wäschereidienste) mit externen Anbietern derselben Leistungen durchgeführt und der Frage nach Eigenerstellung oder Fremdbezug nachgegangen werden.

Planung, Steuerung und Analyse des Leistungsprogramms: Für Zwecke der Planung, Steuerung und Analyse des Leistungsspektrums eines Krankenhauses benötigt es detaillierter Informationen über die Kostensituation in den

${ }^{267}$ Vgl. Haberstock, L. (1998), S. 144-145. 
einzelnen Abteilungen, die ebenfalls in der gewünschten Form besonders die Kostenträgerrechnung bereitstellen kann. ${ }^{268}$

\subsubsection{Identifikation von Kostenträgern}

Im Rahmen der Kostenträgerrechnung werden die Kosten je Bezugsobjekt ermittelt. In Abhängigkeit vom Produktionsprogramm könnten dabei spezifische Kostenträgervarianten gebildet werden.

Im Krankenhausbereich ist die Identifikation des Kostenträgers besonders problematisch. Grundsätzlich besteht die Primärleistung des Krankenhauses in der Verbesserung des Gesundheitszustandes der Patienten. Diese Krankenhausleistung lässt sich nicht quantifizieren und ist somit als Zurechnungsobjekt für Kosten unpraktisch. Im Rahmen der Kostenträgerrechnung ist daher hinsichtlich der Bestimmung der Leistungseinheiten auf Hilfsgrößen zurückgreifen, die sich nicht am Endergebnis, sondern an Zwischenergebnissen bzw. Zwischenprodukten der krankenhausbetrieblichen Leistungserstellung orientieren. ${ }^{269}$ Deswegen ist es für den Krankenhausbetrieb empfehlenswert, nur „Patient einer Krankheitsart“ als sachgerechten Hauptkostenträger zu definieren, dessen Kosten sich sowohl aus krankheitsspezifischen „Kostenbestimmungsfaktoren Patient“ als auch aus den „Kostenbestimmungsfaktoren Pflegetag“ bedingten Teilen zusammensetzen, ${ }^{270}$ wobei Einzelleistungen wie Leistungen im Wirtschafts-, Verwaltungs- und Versorgungsbereich, Leistungen der medizinischen Institutionen, ärztliche Leistungen, Pflegeleistungen und Operativleistungen die Kostenträger sein könnten. ${ }^{271}$

Auf Grund der grundsätzlichen Unterschiedlichkeit der Patienten und der für sie zu erbringenden Leistungen sieht sich der Kostenrechner damit im Kalkulationsproblem einer Einzelfertigung (Patientenkalkulation) gegenüber. Diese erscheint jedoch hinsichtlich ihres Erfassungs- und Durchführungsaufwandes als nicht praktikabel und wirtschaftlich, so dass sie nur in abgeschwächter Form Anwendung findet. Deren Grundlage ist die Klassifizierung der Patientenschaft des Krankenhauses in homogenen Patientkategorien bzw. Fallgruppen (z. B.

\footnotetext{
${ }^{268}$ Vgl. Tscheulin, D. K., Hemig, B. (1994), S. 503-504.

${ }^{269}$ Vgl. Preuß, O. (1996), S. 65.

${ }^{270}$ Vgl. Leonhardt, J. (1988), S. 128.
} 
diagnosebezogenen Fallgruppen, therapiebezogenen Fallgruppen). ${ }^{272}$ Eine Grundlage zur Bildung von Fallgruppen bietet die Internationale Klassifikation der Krankheiten und Verletzungen- „International Classification of Disease ICD“. ${ }^{273}$

\subsubsection{Durchführung der Kostenträgerstückrechnung}

Kostenträger aus Sicht der Kostenrechnung stellen alle betrieblich erstellten Güter und Leistungen dar, die nach Zurechnungsprinzipen zugerechnet werden.

Nach dem Verursachungsprinzip dürfen auf dem Kostenträger nur die Kosten erfasst können die bei einer zusätzlichen Kostenträgereinheit anfallen. Nach dem Durchschnittsprinzip können die Kosten im Durchschnitt auf die Kostenträger zurechnet werden (z. B. Fixkosten). ${ }^{274}$ Aber auch die nicht verursachungsgerechten Kosten dürfen nach dem Tragfähigkeitsprinzip auf die Belastbarkeit der Kostenträger (z. B. auf Grund des erzielbar hohen oder niedrigen Marktpreises) zugeordnet werden.

Die Durchführung der Kostenträgerstückrechnung lässt sich bezüglich des Zeitpunktes in drei verschiedene Kalkulationsarten unterscheiden:

- Vorkalkulation: Eine Vorkalkulation ist eine im Vorhinein durchgeführte Kostenträgerstückrechnung für bestimmte Leistungen, die als Grundlage für

${ }^{271}$ Vgl. Hildbrand, R.: (1988), S. 418.

272 Die Homogenität einer Patientkategorie oder Fallgruppe bedeutet, dass ein jeweils weitgehend übereinstimmendes Spektrum von zu erbringenden Krankenhausleistungen unterstellt werden kann (weitgehend Kostenstrukturgleichheit), und zum anderen eine gewisse Mächtigkeit der Gruppe, die deren Behandlung als selbständigen Kostenträger überhaupt lohnt (Wesentlichkeit). Vgl. Maltry, H., Strehlau-Schwoll, H. (1997), S. 547.

273 Es werden nun 23 Hauptdiagnosekategorien in den USA nach „Diagnosis Related Groups“ (DRG) verwendet. Sie wurden so gestaltet, dass jeweils eine Gruppe von Patienten mit ähnlicher Versorgung und einem vorhersehbaren Umfang an Leistungen und Ressourcenbedarf dargestellt wird. Die Zuordnung der Patienten in die DRGs wird nach Merkmalen wie HauptEntlassungsdiagnose, primäre Operationsmethode, Risikofaktoren und Alter des Patienten oder Entlassungszustand vorgenommen. Auch wurden 47 Krankheitsartengruppen nach „Patient Management Categories“ (PMC) erarbeitet, aus denen wiederum 840 PMC entwickelt wurden. Die Definition der Fallgruppen wurde mit Hilfe des medizinischen Sachverstandes festgelegt. Als Maßstab für die Einordnung der Patienten in die PMC dienten typische Diagnosekombinationen. Vgl. Keun, F. (1997), S. 183-189. Und Kropf, R.(1994): Physician Cost Variance Analysis under DRGs, in: Finkler, S. A. (Edit.),Issues in Cost Accounting for Health Care Organisations, New York, 1994, S. 167-170.

274 Die Fixkosten können nicht nach dem Verursachungsprinzip verursachungsgerecht zugeordnet werden, sondern sie können nach dem Durchschnittsprinzip in den Einproduktsbetriebsfällen mit Hilfe einer Schlüsselgroßen vorgenommen werden. Vgl. Keun, F. (1997), S. 169. 
Preisverhandlungen dient. Mit Hilfe von Vorkalkulationen wird über Aufnahme oder Ablehnung von Aufträgen entschieden.

- Nachkalkulation: Unter einer Nachkalkulation versteht man eine im Nachhinein durchgeführte Kalkulation. Ihre Ergebnisse dienen zum einen der stückbezogenen Erfolgsermittlung und -kontrolle im Rahmen der kurzfristigen Erfolgsrechnung, indem die Ist-Kosten der in einer Abrechnungsperiode erstellten Leistungen bzw. Leistungseinheiten ermittelt werden. Zum anderen dienen die in der Nachkalkulation ermittelten Kosten als Grundlage für zukünftige Vorkalkulationen bei ähnlichen Erzeugnissen. ${ }^{275}$

- Plankalkulation: Unter einer Plankalkulation schließlich ist eine Kostenträgerstückrechnung $\mathrm{zu}$ verstehen, bei der für eine bestimmte Planungsperiode im Voraus geplante Herstell- und Selbstkosten pro Leistungseinheit ermittelt werden.

Die Anwendung der Plankalkulation in der Kostenträgerrechnung im Krankenhaus gering bleibt, zum einen, weil die Planbarkeit von Krankenhausleistungen nicht immer möglich ist - wie bei anderen Fertigungsbetrieben - und die Kostenträgerrechnung damit auch nur bereits Geschehenes dokumentieren kann. Zum anderen, weil die leistungsbezogene Erlös- und Plandaten wegen der großen Bereitstellung von Krankenhausleistungen nicht zu Verfügung stehen. ${ }^{276}$

Schließlich unterscheidet man noch nach dem Umfang der auf die Kostenträger verrechneten Kosten in Vollkostenkalkulationen und Grenzkostenkalkulationen, obwohl praktisch die meisten Krankenhäuser mit der traditionellen Vollkostenrechnung arbeiten. ${ }^{277}$

Die Kostenträgerstückrechnung kann mittels drei verschiedener Hauptkalkulationsverfahren durchgeführt werden: Divisionsverfahren, Zuschlagsverfahren und Kuppelverfahren, die vom Leistungsprogramm und anderen speziellen Merk-

\footnotetext{
${ }^{275}$ Vgl. Freidank, C, (1994), S. 148.

276 Vgl. Richter, H. (1994): Kostenträgerrechnung und interne Budgetierung ab 1995/1996, neue Formen der Kosten- und Leistungsplanung, in: das Krankenhaus, 6/ 1994, S. 258.

277 Vgl. Tscheulin, D. K., Hemig, B. (1997): Aufbau einer Kostenträgerrechnung im Krankenhaus, in: das Krankenhaus, 11/ 1997, S. 503.
} 
malen der verschiedenen Branchen geprägt sind. ${ }^{278}$ In der Praxis werden in verschiedenen Formen verwendet (Abb. 22).

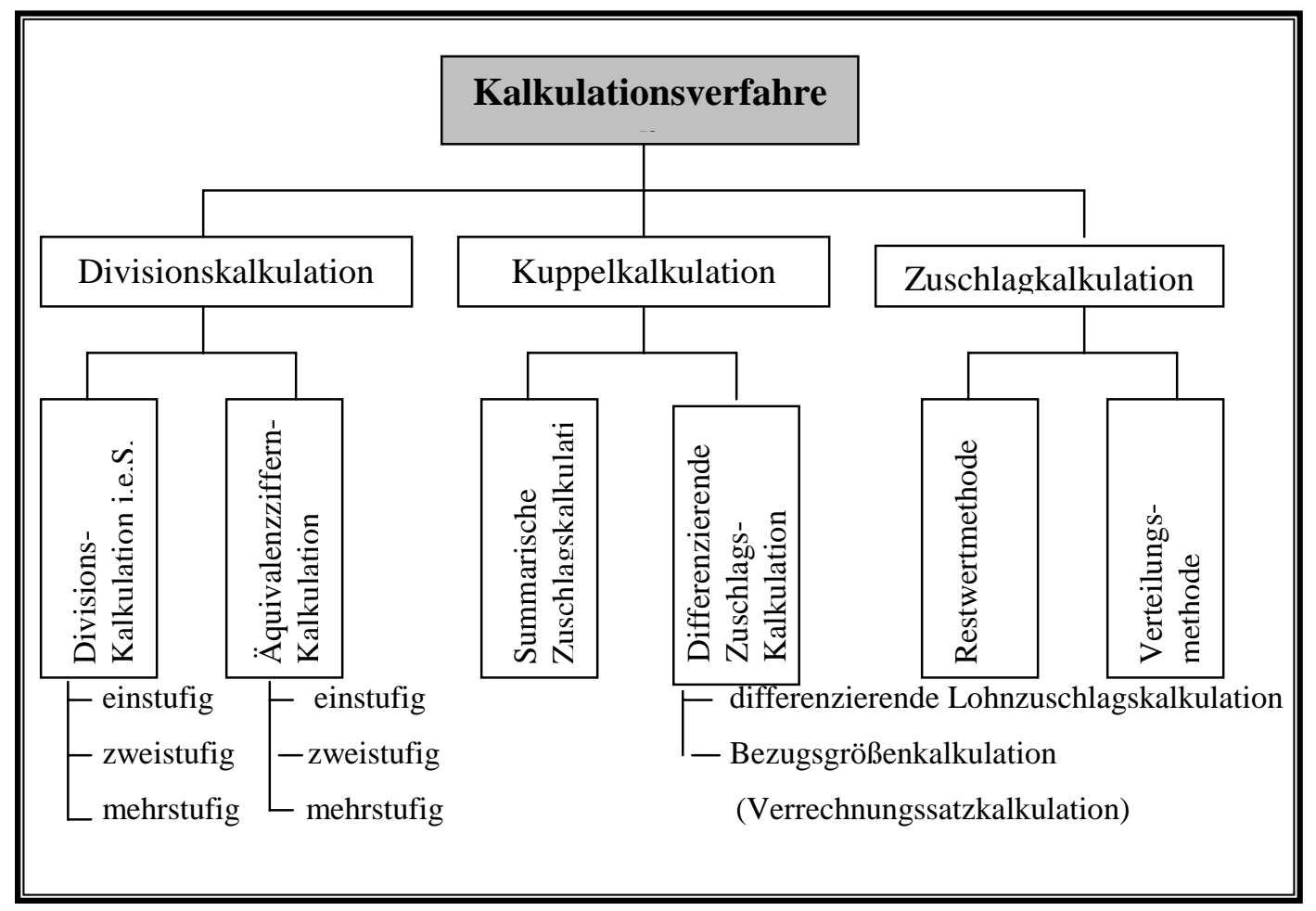

Abb. 22: Systematik der Kalkulationsverfahren. ${ }^{279}$

Der Divisionskalkulation liegt eine Durchschnittsbetrachtung zugrunde, wobei die Gesamtkosten des Betriebes oder einzelner Betriebsbereich durch die Menge der hergestellten Produkte bzw. Leistungen dividiert. Die Divisionskalkulation ist für einen Einprodukt-Betrieb (bzw. Betriebsbereich) entwickelt worden. ${ }^{280}$

Die Äquivalenzziffern-Kalkulation ist eine Unterform der Divisionskalkulation. Allerdings da Sie in Betrieben angewendet wird, in denen nicht einheitliche sondern Produktionssorten bzw. artverwandte Leistungen erstellt werden, soll die Kosten der Leistungen aufgrund der arbeitsablauftechnischen Ähnlichkeiten in

\footnotetext{
${ }^{278}$ Von der Kostenstellenleistung hängt es ab, wie und in welcher Dimension diese zu messen ist. Im Krankenhaus werden grundsätzlich Personalleistungen (meßbar in Zeitgrößen) und Sachleistungen (meßbar in Stück) unterschieden.

${ }^{279}$ Quelle: Tscheulin, D. K., Hemig, B. (1994), S. 504

280 Divisionskalkulationen können mit verschiedenen Varianten verwendet (einstufige oder mehrstufige Divisionskalkulation) angewendet werden. Vgl. Coenenberg, A., (1992), S. 93-96.
} 
einem bestimmten Verhältnis, das in Form von Äquivalenzziffern beobachtet wird, zueinander stehen. ${ }^{281}$

In den Zuschlagskalkulationen werden die Gesamtkosten in Kostenträgereinzelkosten und Kostenträgergemeinkosten getrennt. Während die Kostenträgereinzelkosten dann direkt den Kostenträgern zugeordnet werden können, müssen die Kostenträgergemeinkosten (summarisch oder differenziert) mit Hilfe von Kalkulationssätzen für mehrere Kostenträger zugeschlagen werden. Dies Verfahren kommt zur Anwendung, wenn das Leistungsprogramm in mehrstufigen Abläufen (Einzel- oder Serienfertigung) erstellt wird und dabei die Kosten heterogen verursacht werden, und eine Kostenträgerrechnung in Form der Divisionskalkulation nicht möglich ist. ${ }^{282}$

Die Kuppelkalkulation wird in denjenigen Betrieben verwendet, die aus natürlichen oder technischen Gründen zwangsläufig verschiedene Produkte herstellten. Hiermit sollen die Gesamtkosten des Kuppelproduktionsprozesses nach der Verteilungsoder Restkalkulationsmethode auf die Trägereinheiten (die einzelnen Kuppelprodukte) verteilt werden. ${ }^{283}$

Im Krankenhaus ist die Divisionskalkulationen in aufgrund der Heterogenität der Krankenhausleistungen kaum relevant. Hingegen da sind die Zuschlagskalkulationen dem Krankenhaus von großer Bedeutung, die Leistungserstellung im Krankenhaus in vielen Bereichen mit der Einzel- oder Serienfertigung vergleichbar ist. ${ }^{284}$

\subsubsection{Durchführung der Kostenträgerzeitrechnung}

Die Kostenträgerzeitrechnung ist das letzte Glied einer geschlossenen Betriebsabrechnung, in der die während einer Abrechnungsperiode angefallenen Kosten auf die erstellten Kostenträger umgerechnet werden. Durch Einbeziehung der wert-

\footnotetext{
281 Die Schwierigkeit der Anwendung der Äquivalenzziffernkalkulation besteht in der Ermittlung der Äquivalenzziffern. Grundlage hierfür kann die detaillierte Analyse der Betriebsprozesse sein. Vgl. Haberstock, L. (1998), S. 142.

282 Vgl. Freidank, C, (1994), S. 157-163.

283 Vgl. Haberstock, L. (1998), S. 166-170.

284. Es kann im Krankenhausbereich nach Ziffern wie z. B. Gewichtseinheiten, Zeiteinheiten oder Volumeneinheiten eingesetzt werden. Vgl. Tscheulin, D. K., Hemig, B. (1994), S. 506-507.
} 
mäßigen Leistungen dieser Kostenträger wird die Kostenträgerzeitrechnung häufig zu einer kurzfristigen Erfolgsrechnung (Betriebsergebnisrechnung) erweitert. Das stellt Abb. 23 dar.

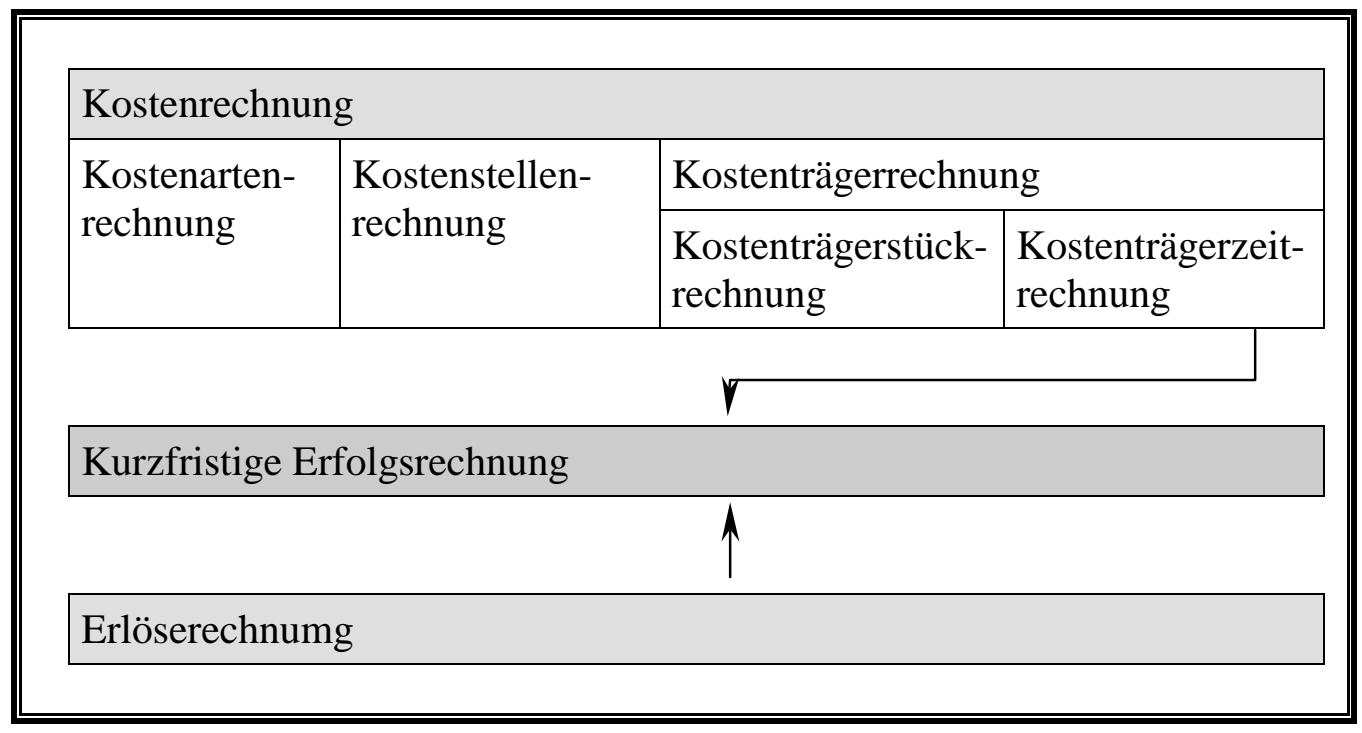

Abb. 23: die kurzfristige Erfolgsrechnung als erweiterte Kostenträgerzeit-
rechnung. Bei Unternehmungen, die nach dem erwerbswirtschaftlichen Prinzip im Interesse der Gewinnerzielung handeln, ist die Betriebsabrechnung das wichtigste Instrument zur Messung des Zielerreichungsgrades der Unternehmung.

Aber in den nicht erwerbswirtschaftlich orientierten und auf die Zielsetzung der Kostendeckung verpflichteten Krankenhäusern mit relativ starrem Leistungsprogramm dient die Betriebsergebnisrechnung im Wesentlichen zur Kontrolle der Selbstkostendeckung. ${ }^{286}$ Insbesondere mit den zunehmend ausgeschöpften Kostensenkungspotentialen spielt die Erlösrechnung als Basisrechnung für die Kostenträgerzeitrechnung eine entscheidende Rolle bei der Beeinflussung des Betriebsergebnisses. $^{287}$

Die Kostenträgerzeitrechnung kann entsprechend der Kostengliederung in zwei Verfahren (Gesamtkostenverfahren und Umsatzkostenverfahren) durchgeführt werden.

\footnotetext{
285 Quelle: Hentze, J., Kehres, E. (1995), 145.

${ }^{286}$ Vgl. Bopp, M., Hohenbild, R. (1984), S. 180.

${ }^{287}$ Vgl. Gabele, E., Fischer, P. (1992), S. 199.
} 
- Im Gesamtkostenverfahren werden die nach Kostenarten gegliederten Gesamtkosten und die nach Kostenträgern gegliederten Gesamterlöse eines Krankenhauses entsprechend der Gewinn- und Verlustrechnung gegenübergestellt.

- Im Umsatzkostenverfahren erfolgt eine Gegenüberstellung von Kostenträgererlösen und Kostenträgerstückkosten bezüglich der abgesetzten Produkte und somit eine Offenlegung der Erfolgsbeiträge der Kostenträger. Die Anwendung dieses Verfahrens erlaubt einen differenzierten Ausweis der einzelnen Erlösarten und Zuordnung der dafür entstandenen Kosten. ${ }^{288}$

\subsubsection{Leistungsrechnung}

\subsubsection{Definition und Aufgaben der Leistungsrechnung}

Die Leistungsrechnung stellt - ebenso wie die Kostenrechnung - ein wichtiges Informationsinstrument für die Betriebsleitung dar. Sie dient in Verbindung mit der Kostenrechnung der Ermittlung des Betriebsergebnisses, der Preisbildung sowie der Planung, Steuerung und Kostenkontrolle des Betriebsprozesses. Hierzu können Istleistungs-, Normalleistungs- oder Planleistungsrechnungen erforderlich sein. ${ }^{289}$

Die Erfassung der Leistungen und Durchführung der Leistungsrechnung ist für die Durchführung der Kosten- und Leistungsanalyse erforderlich. Sie ermöglicht nämlich zum einen die Durchführung einer verursachungsgemäßen und vollständigen innerbetrieblichen Leistungsverrechnung. Zum anderen erlaubt sie auch die Beurteilung der Leistungsfähigkeit der Stellen, deren Daten in die Gesamtbeurteilung des krankenhausbetrieblichen Leistungsprozesses eingehen. Tatsächlich wird in den meisten Krankenhäusern eine Erfassung der Leistungen nur für statistische Zwecke betrieben. Ferner ist in dem Ist-Zustand der vielen öffentlichen Krankenhäusern zu sehen, dass keine Leistungserfassung stattfindet. ${ }^{290}$

\footnotetext{
${ }^{288}$ Vgl. Maltry, H., Strehlau-Schwoll, H. (1997), S. 551.

${ }^{289}$ Vgl. Kosiol, E. (1979): Kosten- und Leistungsrechnung, Berlin, 1979, S. 137.

${ }^{290}$ Vgl. Schwarz, R. (1993), S.117.
} 


\subsubsection{Durchführung der Leistungsrechnung}

Analog zur Kostenrechnung wird zunächst eine Leistungsartenrechnung durchgeführt, in der die realisierten Leistungen differenziert nach Art der erstellten Leistungen erfaßt werden. In der Leistungsstellenrechnung werden die Leistungen den Orten ihrer Entstehung zugeordnet. Besonders in Krankenhäusern können viele Leistungsarten eindeutig bestimmten Leistungsstellen zugeordnet werden und mit einer Leistungsstellenrechnung entscheidungsrelevante Informationen gewonnen werden. ${ }^{291}$

Die Durchführung einer Leistungsträgerrechnung ist mit der Leistungsartenrechnung identisch, und Leistungsträger sind die Arten der erstellten Leistungen, deswegen erübrigt sie sich, und die Leistungsträger werden in der Leistungsartenrechnung erfasst. ${ }^{292}$

Um die Aufgaben der Kosten- und Leistungsrechnung im Krankenhaus zu erfüllen, ist die Leistungsdokumentation nicht nur je Leistungsträger vorzunehmen, sondern muss in der endgültigen Ausgestaltung ,just in time“ erfolgen. Dies bedeutet, dass während des Leistungserstellungsprozesses (während der Patient im Krankenhaus ist) die Leistungen bzw. die Leistungsanforderungen dokumentiert werden müssen. Hierzu stellt sich in der Krankenhauspraxis, insbesondere bei Notfällen, häufig das Problem, dass zum Zeitpunkt der Leistungserbringung noch nicht festgestellt werden kann, ob die Behandlung unter ambulanter oder stationärer Aufnahme erfolgen müsste. ${ }^{293}$

\subsubsection{Erfassung der Leistungsarten und Leistungsstatistik}

Die Erfassung der Leistungsarten muss analog zu den Kostenarten an den Orten erfolgen, wo sie erstellt worden sind. Die Qualität der Leistungsrechnung im

\footnotetext{
${ }^{291}$ Im Allgemeinen werden Leistungsstellenrechnungen nicht durchgeführt, da einerseits die Kosten der gewonnenen Informationen oft höher als der Wert der Information bzw. ihr Nutzen für betriebliche Entscheidungen über leistungsrelevante Sachverhalte sind. Anderseits, da meistens mehrere Abteilungen an der Erstellung einer Leistungsart beteiligt sind, ist es nur selten möglich, Bezugsgrößen zu definieren, mit denen Leistungsarten eindeutig bestimmten Leistungsstellen zugeordnet werden können. Vgl. Bölke, G., Schmidt- Rettig, B. (1988): LeistungsrechnungLeistungsstatistik: in: Eichhorn, S. (Hrsg.), Handbuch Krankenhausrechnungswesen, GrundlagenVerfahren- Anwendungen, 2. Aufl., Wiesbaden, 1988, S. 459.

${ }^{292}$ Leistungsarten bzw. Leistungsträger sind wiederum gleichzeitig Kostenträger, denen die Kosten als sachzielbezogene bewertete Güterverbräuche zugeordnet werden. Das könnte die Ursache dafür sein, dass die Leistungsrechnung nicht eigenständig sondern als integraler Bestandteil einer Kostenrechnungstheorie aufgefaßt würde.
} 
Krankenhaus wird durch den konzeptionellen Ansatz, insbesondere die sachgerechte Leistungsdefinition und fehlerfreie Erfassung erreicht. In den Krankenhäusern liegen die Schwierigkeiten der mengengemäßen Leistungserfassung, einerseits in der Unterschiedlichkeit der Leistungsarten zwischen den verschiedenen Krankenhausbereichen und andererseits zwischen den Leistungsstellen innerhalb eines Bereiches. Deswegen werden im Krankenhaus verschiedene Leistungserfassungs- und Leistungsauswertungstechniken kombiniert. Beispielsweise müssen bei der Bewertung der erfassten Leistungen eines Krankenhausbereichs die verschiedenartigen Leistungen mit Äquivalenzziffern bewertet werden, wenn sie zu einer Gesamtleistungszahl je Leistungsstelle zusammengefasst werden sollen. ${ }^{294}$ Zur Einführung einer von denen sind Faktoren wie Arbeitsaufwand, Fehlerquoten und Flexibilität mit Kosten- Nutzen- Analyse zu berücksichtigen. ${ }^{295}$

Zur Erfassung der Leistungen bedarf es generell des Aufbaus einer umfassenden Leistungsstatistik, wobei auf die technischen Möglichkeiten und auf das wirtschaftlich sinnvolle Rücksicht zu nehmen ist.

Als Krankenhaus-Leistungsdaten können die in den einzelnen Statistiken folgende aufgenommen werden:

- medizinische Leistungsdaten: Art und Zahl der diagnostischen und therapeutischen Leistungen einschließlich der Untersuchungsbefunde und Behandlungsergebnisse,

- pflegerische Leistungsdaten: Art und Zahl der Leistungen im Bereich von Grund- und Behandlungspflege einschließlich der Protokollierung des Patientenbefundes und

- $\quad$ allgemeine Leistungsdaten: Art und Zahl der Versorgungs- und Verwaltungsleistungen.

${ }^{293}$ Vgl. Strehlau-Schwoll, H. (1993), S. 216.

294 Äquivalenzziffern als Verhältniszahlen, die die unterschiedlichen Kosten je Leistungseinheit der verschiedenen Leistungsarten wiederspiegeln sollen, können betriebsindividuell ermittelt werden oder von überbetrieblichen Äquivalenzziffernsystemen übernommen werden. Vgl. Bopp, M., Hohenbild, R. (1984), S. 170-172.

295 Grundsätzlich bestehen die Möglichkeiten für die Leistungserfassung wie manuelle Erfassung bzw. Auswertung, Tastaturerfassung, Belegleser oder Barcode. Vgl. Hentze, J., Kehres, E. (1995), S. 92-93. 
Mit dieser Leistungsstatistik können zum einen Kennzahlen, die über einen operationalen, quantifizierbaren, zahlmäßig erfassbaren betriebswirtschaftlichen Tatbestand informieren, gebildet werden. ${ }^{296}$ Diese Leistungsstatistik dient sowohl intern als auch extern Informationsabnehmern, da sie als Grundlage für dispositive Aufgaben und für die Beurteilung der Leistungsfähigkeit einzelner Bereiche sowie des gesamten Krankenhauses fungieren können. Zum anderen dient ein Teil dieser Leistungsstatistik auch als Grundlage für die Verrechnung von innerbetrieblichen Leistungen und geht somit in die Leistungsarten- und Leistungsstellenrechnung der kostenstellenorientierten Erfolgsrechnung ein. ${ }^{297}$

\subsection{Teilgebiete der Kosten- und Leistungsrechnung in deutschen Kranken- häusern}

In diesem Abschnitt werden die Teilgebiete der Kosten- und Leistungsrechnung unter Betrachtung ihrer aktuellen Anwendung in der Bundesrepublik Deutschland behandelt.

Die Kosten- und Leistungsrechnung im deutschen Krankenhaus ist allgemein im $\S 8$ Krankenhausbuchführungsverordnung (KHBV) vorgeschrieben. Gemäß den Mindestanforderungen ${ }^{298}$ sind Kostenstellen aufgrund ihrer Aufgaben und Strukturen zu bilden, mindestens jedoch solche Kostenstellen, die sich aus den Daten der Kostenstellenrahmen nach Anlage 5 ergeben, sofern hierfür Kosten und Leistungen anfallen, Kosten aus der Buchführung herzuleiten und Kosten und Leistungen nach Kostenstellen verursachungsgerecht zu erfassen.

\footnotetext{
${ }^{296}$ Für weitere Details über Kennzahlen vgl. Eichhorn, S. (1987) S. 60-77.

${ }^{297}$ Vgl. Eichhorn, S. (1976), S. 112-115, sowie Eichhorn, S. (1987), S. 98-103.

298 Mindestanforderungen an die Kosten- und Leistungsrechnung für die deutschen Krankenhäuser sind im Abschnitt 3.2.4 dargelegt worden.
} 


\subsubsection{Kostenartenrechnung in den deutschen Krankenhäusern}

\subsubsection{Definition und Gliederung der Kostenartenrechnung in den deutschen Krankenhäusern}

Abgesehen von der Möglichkeit und Notwendigkeit, sowohl für die Geschäftsbuchhaltung als auch für die Kosten- und Leistungsrechnung weiter Unterteilungen der Kostenarten-Konten vorzunehmen, wird auch bei den deutschen

Krankenhäusern gesetzlich ein Kontenrahmen vorgegeben. Die Einteilung bzw. Gliederung der Kostenarten in den deutschen Krankenhäusern ergibt sich aus den Bestimmungen der (KHBV), insbesondere aus $\S 3 \mathrm{KHBV}$ im Verbindung mit dem Kontenrahmen, der als Anlage 4 Bestandteil der KHBV ist und damit Verordnungscharakter hat. Mit der berechtigten Forderung, im Krankenhausbereich eine aussagefähige Kostenartenrechnung zu führen, wird es zur Erfüllung vorstehend genannter Gliederungsgesichtspunkte notwendig, auch auf Seiten des Gesetzgebers über einen Ausbau der Kostenarten im Kontenrahmen nachzudenken, zumal dies auch für die Aussagefähigkeit der Kostenstellen- und Kostenträgerrechnung zwangsläufig von großer Bedeutung ist. ${ }^{299}$

Da die Kosten eines Krankenhauses nach $\S 8$ KHBV aus der Buchführung nachprüfbar abzuleiten sein müssen, empfiehlt sich in Anlehnung an den in Form eines Mindestgliederungsschemas vorgegebenen Kontenrahmen für die Buchführung eine Gliederung der Kostenarten nach Produktionsfaktoren. Wenn die Kosten aus der Finanzbuchhaltung nachprüfbar herzuleiten sind, bedeutet das für die Praxis gleichzeitig, dass die Finanzbuchhaltung die Werte auf den dort verarbeiteten Kostenarten-Konten der Kostenrechnung bereitstellt.

Der Kostenstamm, der aus der Finanzbuchhaltung übernommen wird, ist so aufgebaut, dass die Zuordnung der Kostenarten über den Kontenplan erfolgen kann. ${ }^{300}$ Der Kontenrahmen als allgemeingültiges Gliederungsschema ist für jedes Krankenhaus Grundlage für die Entwicklung seines individuellen Kontenplanes. d. h. im

\footnotetext{
${ }^{299}$ Vgl. Klockhaus, H. E. (1997), S. 38.

300 Vgl. Juretzka, G. (1989): Aufbau eines internen Rechnungswesens am Universitätsklinikum Göttingen, in: das Krankenhaus, 11/ 1989, S. 591.
} 
Rahmen des Musterkontenplans nach der KHBV 1978 und den Änderungen durch BPflV $1995^{301}$ kann jedes Krankenhaus je nach Anforderungen eine individuelle Ausgestaltung in der Weise vornehmen, so dass eine Überleitung auf die Konten des vorgeschriebenen Kontenrahmens jederzeit möglich ist. ${ }^{302}$ Bei der Ausgestaltung des individuellen Kontenplans ist neben der Einhaltung der gesetzlichen Mindestanforderungen darauf zu achten, dass er in einfacher Weise eine Abteilung der pflegesatzfähigen Kosten und deren Zuordnung $\mathrm{zu}$ den Positionen der Leistungs- und Kalkulationsaufstellung ermöglicht muß. ${ }^{303}$

Nach dem Musterkontenplan werden die Konten und deren Inhalte einheitlich definiert. Damit wird die klar und einheitlich geordnete Leistungs- und Kalkulationsaufstellung den Vergleich mit anderen Krankenhäusern leicht ermöglicht.

Der Musterkontenplan umfasst 10 Kontenklassen, wobei jede von denen nach Kontengruppen, Kontenuntergruppen und Konten differenziert wird. Durch die Gliederung der Aufwendungen in die Klassen 6 und 7 ist die Struktur der aufwandgleichen Kostenarten bereits vorgegeben. Sie soll wegen der dortigen Gliederung nach Art der verbrauchten Produktionsfaktoren und der damit verbundenen Transparenz über die verwandten Einsatzgüter beibehalten werden. In der Kontenklasse 6 sind Aufwendungen erfasst, die gleichzeitig pflegesatzrelevante Kosten darstellen und ebenso ohne Ausnahme in die Kostenrechnung übernommen werden. Tab. 7 stellt den Kontenrahmen (Aufwand- bzw. Kostenartengruppen) entsprechend Anlage 4 zur KHBV dar:

Bei diesem Schema ist zu beachten, dass die Kostenarten, die im Rahmen der Kontrolle besondere Bedeutung haben (z. B. Personalkosten, Kontengruppen 60 64), detailliert zu gliedern sind. Weiterhin sind alle die Positionen aus dem Kontenrahmen zu eliminieren, die neutrale Aufwendungen betreffen (außerordentliche Aufwendungen Kontengruppe 79), die weitgehend der klassischen

\footnotetext{
301 Die Systematik des Kontenplanes orientiert sich gleichzeitig an den Anforderungen des Kostenund Leistungsnachweises, der 1995 von der Leistungs- und Kalkulationsaufstellung abgelöst wurde.

302 Vgl. Keun, F. (1997), S. 103.

${ }^{303}$ Vgl. Bundesministerium für Gesundheit (1995): Leitfaden zur Einführung von Fallpauschalen und Sonderentgelten gemäß BPflV 1995: Datenbedarf, Kalkulationsgrundlagen, Abrechnungsmodalitäten, Kostengliederung, Schriftenreihe des Bundesministeriums für Gesundheit, Band 44, Baaden- Baaden, 1995.S. 79.
} 
Gliederung der neutralen Aufwendungen in außerordentliche, periodefremde und betriebsfremde entsprechen. ${ }^{304}$

\begin{tabular}{|c|c|c|c|}
\hline $\begin{array}{l}\text { Konten- } \\
\text { klasse }\end{array}$ & $\begin{array}{l}\text { Konten- } \\
\text { gruppe } \\
\text { n }\end{array}$ & Kostenarten (Aufwendungen) & $\underset{305}{\text { Struktur }}$ \\
\hline \multirow[t]{10}{*}{6} & 60 & Löhne und Gehälter & \multirow{5}{*}{$67 \%$} \\
\hline & 61 & Gesetzliche Sozialabgaben & \\
\hline & 62 & Aufwendungen für die Altersversorgung & \\
\hline & 63 & $\begin{array}{l}\text { Aufwendungen für die Beihilfen und } \\
\text { Unterstützungen }\end{array}$ & \\
\hline & 64 & Sonstige Personalaufwendungen & \\
\hline & 65 & Lebensmittel & $3 \%$ \\
\hline & 66 & Medizinischer Bedarf & $16 \%$ \\
\hline & 67 & Wasser, Energie, Brennstoff & $3 \%$ \\
\hline & 67 & Wirtschaftsbedarf & $3 \%$ \\
\hline & 69 & Verwaltungsbedarf & $2 \%$ \\
\hline \multirow[t]{7}{*}{7} & 70 & Aufwendungen für zentrale Dienstleistungen & $1 \%$ \\
\hline & 71 & Wiederbeschaffte Gebrauchsgüter & $0 \%$ \\
\hline & 72 & Instandhaltung & $3 \%$ \\
\hline & 73 & Steuern, Abgaben, Versicherungen & $1 \%$ \\
\hline & 74 & Zinsen und ähnliche Aufwendungen & - \\
\hline & 78 & Sonstige ordentliche Aufwendungen & \multirow[t]{2}{*}{$1 \%$} \\
\hline & 79 & Außerordentliche Aufwendungen & \\
\hline & & & $100 \%$ \\
\hline
\end{tabular}

Tab. 7: Kostenarten und Kostenstruktur. ${ }^{306}$

\subsubsection{Erfassung der Kostenartenrechnung im deutschen Krankenhaus}

Die im Musterkontenrahmen vorgelegten Kostenarten weisen bereits eine vergleichsweise große Gliederungstiefe auf, die nur dann weiter differenziert wer-

\footnotetext{
304 Vgl. Leonhardt, J. (1988), S. 79.

305 Vgl. Deutsche Krankenhausgesellschaft (1990): Auswertung von Kosten- und Leistungsnachweisen 1988, Düsseldorf, 1990, S. 10.

306 Quelle: Anlag 4 KHBV.
} 
den sollte, wenn dies aus Finanzierungsgründen oder für Zwecke der betrieblichen Feinsteuerung zweckmäßig ist. ${ }^{307}$

Diese Kostenarten werden differenziert nach der Mengen- und Preiskomponente (getrennte Erfassung) oder undifferenziert erfasst. Für die betriebsinterne Steuerung und Beurteilung der Wirtschaftlichkeit ist eine getrennte Erfassung der Wert- und Mengenkomponente unverzichtbar, die aufgrund der Anforderungen an die Leistungs- und Kalkulationsaufstellung für jedes Krankenhaus Pflicht ist. ${ }^{308}$

- Personalkosten:

Personalkosten sind alle Kosten, die durch den Personaleinsatz mittelbar oder unmittelbar entstehen. Sie stellen mit einem Anteil von zwei Dritteln den größten Kostenblock im deutschen Krankenhaus dar, dazu gehören Lohn- und Gehaltskosten sowie Personalnebenkosten. Sie werden im Wesentlichen in der Lohn- und Gehaltsbuchhaltung ermittelt und dann auf Sammelkonten der Kontenklasse 6 der Finanzbuchhaltung übertragen, das bedeutet, die Personalkostenarten, die in KHBV Anlage 4 und in der Selbstkostenplatte nach $\S 18$ Abs. 2 BPflV aufgeführt werden, sind Grundkosten, die direkt aus der Finanzbuchhaltung als Zweckaufwand in die Kostenrechnung übernommen können. Mit der dritten Änderungsverordnung zur KHBV wurden zwar die Zuordnungsvorschriften für die Dienstarten der Personalkosten geändert, jedoch wurde keine neue Aufteilung und Untergliederung der Kostenarten vorgenommen.

Es ist davon auszugehen, dass man entsprechende Erfahrungen in der Anwendung der Kosten- und Leistungsrechnung sammelt und dann auch in der KHBV nachholt, die Kostenarten der Personalkosten neu festzulegen. ${ }^{309}$ Tab. 8 stellt die Dienstarten der Personalkosten und ihre Struktur dar:

\footnotetext{
307 Vgl. Hildbrand, R. (1988), S. 398.

308 Die Pflicht zur Mengenerfassung ergibt sich aus dem Aufbau der Systematik der Leistungs- und Kalkulationsaufstellung, für die in Teil L2 (Personal des Krankenhauses) die durchschnittlich beschäftigten Vollkräfte, differenziert nach Dienstarten für den laufenden und den folgenden Pflegesatzzeitraum, anzugeben sind. Vgl. Hentze, J., Kehres, E. (1995), S. 49, und L2 Anlage 3 zu $§ 17$ Abs. 4 BPflV.

${ }^{309}$ Vgl. Klockhaus, H. E. (1997), S. 39.
} 


\begin{tabular}{||l|l|}
\hline Dienstarten der Personalkosten & Struktur $^{310}$ \\
\hline Ärztlicher Dienst & $22 \%$ \\
\hline Pflegedienst & $34 \%$ \\
\hline Medizinisch- technischer Dienst & $11 \%$ \\
\hline Funktionsdienst & $9 \%$ \\
\hline Klinisches Hauspersonal & $4 \%$ \\
\hline Wirtschafts- und Versorgungsdienst & $9 \%$ \\
\hline Technischer Dienst & $2 \%$ \\
\hline Verwaltungsdienst & $6 \%$ \\
\hline Sonderdienst & $1 \%$ \\
\hline Personal der Ausbildungsstätten & $1 \%$ \\
\hline Sonstiges Personal & $1 \%$ \\
\hline & $100 \%$ \\
\hline
\end{tabular}

Tab. 8 : Dienstarten der Personalkosten. ${ }^{311}$

Der Kostenrahmen der Anlage 4 zur KHBV unterscheidet Personalkostenarten, wobei innerhalb der Kontengruppen für Personalkostenarten jeweils nach Dienstarten differenziert wird. Beispielsweise stellt die Tab. 9 die Differenzierung die Löhne und Gehälter:

\begin{tabular}{|c|c|c|}
\hline \multirow{10}{*}{$\begin{array}{l}6 \\
\text { Personal } \\
\text { kosten }\end{array}$} & \multirow{10}{*}{$\begin{array}{l}60 \text { Löhne und } \\
\text { Gehälter }\end{array}$} & 6000 Vergütung an Ärztlicher Dienst \\
\hline & & 6001 Vergütung an Pflegedienst \\
\hline & & $\begin{array}{l}6002 \text { Vergütung an Medizinisch- technischer } \\
\text { Dienst }\end{array}$ \\
\hline & & 6003 Vergütung an Funktionsdienst \\
\hline & & 6004 Vergütung an Klinisches Hauspersonal \\
\hline & & $\begin{array}{l}6005 \quad \text { Vergütung an Wirtschafts- und } \\
\text { Versorgungsdienst }\end{array}$ \\
\hline & & 6006 Vergütung an Technischer Dienst \\
\hline & & 6007 Vergütung an Verwaltungsdienst \\
\hline & & 6008 Vergütung an Sonderdienst \\
\hline & & $\begin{array}{l}6010 \quad \text { Vergütung an Personal der } \\
\text { Ausbildungsstätten }\end{array}$ \\
\hline
\end{tabular}

\footnotetext{
${ }^{310}$ Vgl. Deutsche Krankenhausgesellschaft (1990), S. 10.

311 Quelle: Hentze, J., Kehres, E. (1995), S. 52.
} 


\begin{tabular}{|l|l|l||}
\hline & 6011 Vergütung an Sonstiges Personal \\
& $\begin{array}{l}6012 \quad \text { Vergütung an Nicht zurechenbare } \\
\text { Personalkosten }\end{array}$ \\
\hline \hline
\end{tabular}

Tab. 9: Löhne und Gehälter differenziert nach Dienstarten. ${ }^{312}$

In Löhne und Gehälter werden alle Leistungsentgelte einschließlich Vergütung für Überstunden, Bereitschaftsdienst und Rufbereitschaft, Zeitzuschläge, Sachbezüge für freie Unterkunft und Verpflegung sowie Gestellungsgelder erfaßt, ${ }^{313}$ ebenso jährliche Einzahlungen (z. B. Weihnachtsgeld, Urlaubsgeld, Gemeindeunfallversicherung usw. $)^{314}$

Aus der Kostenrechnung der deutschen Krankenhäuser ist zu ersehen, dass einerseits die Löhne im Krankenhaus Hilfslöhne, die Gemeinkosten sind, ausschließen ${ }^{315}$ und andererseits keine Aufteilung der Personalkosten in Löhne und Gehälter vorgenommen, aber nur eine Differenzierung in regelmäßig und unregelmäßig wiederkehrende Zahlungen wie z. B. Zuschläge für Überstunden oder Rufbereitschaft abgefaßt wird. Diese Differenzierung ist sinnvoll um eine Wirtschaftlichkeitskontrolle zu ermöglichen. ${ }^{316}$

In den gesetzlichen Sozialabgaben werden der Arbeitgeberanteil zur Sozialversicherung (Kranken-, Renten, und Arbeitslosenversicherung) sowie die Beiträge zur gesetzlichen Unfallversicherung erfasst.

$\mathrm{Zu}$ den Aufwendungen für die Altersversorgung gehören Beiträge zu Ruhegehaltsund Zusatzversicherungskassen.

Die Aufwendungen für Beihilfen und Unterstützung enthalten alle Beihilfen und Unterstützungen für Mitarbeiter sowie deren Hinterbliebene.

${ }^{312}$ Quelle: Anlag 4 KHBV.

313 Der Wert der freien Unterkunft (Kost und Wohnung) stellt einen Sachbezug im Sinne des $§ 8$ Abs. 2 Einkommensteuergesetz dar und ist entsprechend der Sachbezugsverordnung zu bewerten und unter Position Löhne und Gehälter auszuweisen. Gestellungsgelder sind Vergütungen für Leistungen, insbesondere von Angehörigen von Ordensgemeinschaften, die dem Träger des Ordens zufließen.

${ }^{314}$ Diese jährlichen Einzahlungen sind monatlich abzugrenzen und mit jeweils einem Zwölftel in der Kostenrechnung zu übernehmen.

315 In gewerblichen Betrieben werden die Löhne nach verrechnungstechnischen Gesichtspunkten in Fertigungslöhne und Hilfslöhne differenziert. Fertigungslöhne sind Kosten, die durch den Verbrauch von Arbeitsleistungen entstehen, die unmittelbar zur Fertigung des Produktes beitragen, während Hilfslöhne nur mittelbar bei der Leistungserstellung anfallen. Vgl. Hentze, J. (1979), S. 53.

316 Vgl. Rohrig, R., Schnee, S. (1995): Kostenarten, Kostenstellen, Kostenträgerrechnung, in: Eichhorn, S., Schmidt- Rettig, B. (Hrsg.): Krankenhausmanagement im Werte- und Strukturwandel, Stuttgart, Berlin, Köln, 1995, S. 271. 
Die sonstigen Personalaufwendungen erfassen aufteilbare sonstige Kosten für das Personal, wie Erstattung von Fahrkosten zum Arbeitsplatz, freiwillige Sozialleistungen an die Mitarbeiter (z.B. Jubiläumsgeschenke und -Zahlungen, Weihnachtsgeschenke).

\section{- Sachkosten}

Nach einer Gliederung der Sachkosten wurde in KHBV, in Verbindung mit der neuen Leistungs- und Kalkulationsaufstellung LKA der BPflV 95, wie folgt verlangt(Tab. 10):

\begin{tabular}{|l|l|}
\hline Kostenarten der Sachkosten & Struktur \\
\hline Lebensmittel und bezogene Leistungen & $9 \%$ \\
\hline Medizinischer Bedarf & $49 \%$ \\
\hline Wasser, Energie, Brennstoffe & $9 \%$ \\
\hline Wirtschaftsbedarf & $11 \%$ \\
\hline Verwaltungsbedarf & $5 \%$ \\
\hline Zentrale Verwaltungsdienste & $2 \%$ \\
\hline Zentrale Gemeinschaftsdienst & $1 \%$ \\
\hline Steuern, Abgaben, Versicherungen & $2 \%$ \\
\hline Instandhaltungen & $10 \%$ \\
\hline Gebrauchsgüter & $1 \%$ \\
\hline Sonstiges & $1 \%$ \\
\hline & $100 \%$ \\
\hline \hline
\end{tabular}

Tab. 10: Kostenarten und Struktur der Sachkosten ${ }^{317}$

Im Kontenrahmen erfolgt eine differenzierte Gliederung (Mindestforderung). Darüber hinaus würde sich eine weitere Untergliederung der Kontenuntergruppen für eine leistungsgerechte Kalkulation von Fallpauschalen und Sonderentgelten und für die Erfüllung der Aufgaben der Steuerung und der Wirtschaftlichkeitskontrolle, insbesondere bei sehr kostenintensiven Sachgütern, empfehlen. Da die wirtschaftlich größte Bedeutung innerhalb der Sachkosten der medizinische Sachbedarf hat, ist dessen Differenzierung und Struktur sowie weitere Differenzierung in Tab. 11 dargestellt.

${ }^{317}$ In Anlehnung an: Deutsche Krankenhausgesellschaft (1990), S. 10. 


\begin{tabular}{|c|c|c|c|}
\hline $\begin{array}{l}\text { Konte } \\
\text { n- } \\
\text { grupp } \\
\text { e } 66\end{array}$ & Medizinischer Bedarf & $\begin{array}{l}\text { Weitere Differenzierung } \\
\text { nach ... }\end{array}$ & $\begin{array}{l}\text { jtruk- } \\
\text { ur }\end{array}$ \\
\hline 6600 & $\begin{array}{l}\text { Arzneimittel, Heil- und } \\
\text { Hilfsmittel }\end{array}$ & $\begin{array}{l}\text { Indikationsgruppen (z. B. } \\
\text { Antibiotika, Zytostatika usw.) }\end{array}$ & $29 \%$ \\
\hline 6601 & \multicolumn{2}{|l|}{ Kosten der Lieferapotheke } & $1 \%$ \\
\hline 6602 & $\begin{array}{l}\text { Blut, Blutkonserven und } \\
\text { Blutplasma }\end{array}$ & $\begin{array}{l}\text { Blut, Blutkonserven und } \\
\text { Blutplasma }\end{array}$ & $7 \%$ \\
\hline 6603 & \multicolumn{2}{|c|}{ Verbandsmaterial } & $4 \%$ \\
\hline 6604 & \multicolumn{2}{|c|}{ Ärztliches und pflegerisches Verbrauchsmaterial, Instrumente } & $12 \%$ \\
\hline 6606 & $\begin{array}{l}\text { Narkose- und sonstige OP- } \\
\text { Bedarf }\end{array}$ & $\begin{array}{l}\text { Katheter, Infusionsbestecke, } \\
\text { Kanülen, } \quad \text { Nahtmaterial, } \\
\text { Klammergeräte Skalpelle }\end{array}$ & $11 \%$ \\
\hline 6607 & $\begin{array}{l}\text { Bedarf für Röntgen- und } \\
\text { Nuklearmedizin }\end{array}$ & $\begin{array}{l}\text { Röntgenfilme, Entwickler und } \\
\text { Fixierer, Kontrastmittel, } \\
\text { Katheter und Führungsdrähte }\end{array}$ & $6 \%$ \\
\hline 6608 & \multicolumn{2}{|l|}{ Laborbedarf } & $8 \%$ \\
\hline 6609 & \multicolumn{2}{|c|}{ Untersuchungen in fremden Instituten } & $7 \%$ \\
\hline 6610 & $\begin{array}{l}\text { Bedarf für EKG, EEG, } \\
\text { Sonographie }\end{array}$ & $\begin{array}{l}\text { EKG, EEG, Sonographie, } \\
\text { Sonstiges }\end{array}$ & $1 \%$ \\
\hline 6611 & \multicolumn{2}{|c|}{ Bedarf der Physikalischen Therapie } & $0 \%$ \\
\hline 6612 & \multicolumn{2}{|c|}{ Apothekenbedarf. Desinfektionsmaterial } & $1 \%$ \\
\hline 6613 & Implantate & $\begin{array}{l}\text { Arten der Implantate (z. B. } \\
\text { Hüft- endoprothesen, } \\
\text { Herzschrittmacher }\end{array}$ & $7 \%$ \\
\hline 6614 & \multicolumn{2}{|l|}{ Transplantate } & $0 \%$ \\
\hline 6615 & \multicolumn{2}{|l|}{ Dialysebedarf } & $2 \%$ \\
\hline 6616 & \multicolumn{2}{|l|}{ Kosten für Krankentransporte } & $1 \%$ \\
\hline 6617 & \multicolumn{2}{|l|}{ Sonstiger medizinischer Bedarf } & $3 \%$ \\
\hline & & & $100 \%$ \\
\hline
\end{tabular}

Tab. 11: Differenzierung und Struktur des medizinischen Sachbedarfs ${ }^{318} \#$

318 In Anlehnung an: Deutsche Krankenhausgesellschaft (1990), S. 27. 


\section{- Zinsen:}

Gemäß förderungsspezifischem Recht (KHG, SGB XI) können ebenfalls Fremdkapitalzinsen entstehen (z. B. durch Überbrückungskredite). Betriebsmittelkreditzinsen sind nur zu berücksichtigen, wenn sie zu den pflegesatzfähigen Kosten gehören. $^{319}$

Nach $\S 8$ Abs. 1 BPflV und entsprechend dem dualistischen Finanzierungssystem im Krankenhaus werden Zinsen für Betriebsmittelkredite im Kosten- und Leistungsnachweis und in der Kosten- und Leistungsaufstellung als pflegesatzfähige Kosten durch eine gesonderte Kontoführung nachgewiesen, wenn Kredit erforderlich aufgrund von Liquiditätsengpässen aufgenommen werden und es einer wirtschaftlichen Betriebsführung entspricht, dass das Krankenhaus primär, soweit vorhanden, Eigenmittel einzusetzen hat. Die Notwendigkeit, Betriebsmittelkredite aufzunehmen, soll dadurch eingeschränkt werden, dass die in der BPflV vorgesehenen Möglichkeiten (Teilzahlungen für längere Krankenhausaufenthalte sowie eine zeitnahe Zahlung der Pflegesätze) genutzt werden. ${ }^{320}$

\section{- Abschreibungen:}

Krankenhaus-Betriebsmittel wie z. B. Gebäude, Technische Anlagen, Einrichtungsund Ausstattungsgegenstände sind langfristig nutzbare Anlagegegenstände. Sie werden durch die Nutzung und den Zeitablauf im Wert gemindert. Die Wertminderung (Abschreibung) ist im förderungsspezifischen Recht (KHG, SGB XI) ein Teil der Vorhaltekosten. Und da die Anlagegüter der Krankenhäuser nach dem dualistischen Finanzierungssystem sind zum größten Teil von den Bundesländern finanziert werden, ${ }^{321}$ gehören die Abschreibungen, soweit sie durch öffentliche Mittel gefördert werden, nicht zu den pflegesatzfähigen Kosten. Abschreibungen

\footnotetext{
319 Kosten sind dann pflegesatzfähig, wenn sie nach den Bestimmungen der BPflV über Budget und Pflegesätze gedeckt werden. Vgl. Koch, J. (1998), S. 56.

${ }^{320}$ Vgl. Hentze, J., Kehres, E. (1995), S. 58.

321 Vgl. Gerdelmann, W. (1996): GSG aus Sicht der Krankenkassen, in: Adam, D. (Hrsg.): Krankenhausmanagement, Band 59, SzU., Wiesbaden, 1996, S. 38.
} 
der Gebrauchsgüter der Rationalisierungsinvestitionen entsprechend der $\S 18 \mathrm{~b}$ und $\S 17$ Abs. 4 KHG sind jedoch pflegesatzfähig. ${ }^{322}$

In der Finanzbuchhaltung sind die Abschreibungen nach den Vorschriften der KHBV in Verbindung mit handels- und steuerlichen Vorschriften behandelt worden. Gemäß $\S 5$ Abs. 1 KHBV in Verbindung mit $\S 253$ HGB sind Vermögensgegenstände des Anlagevermögens, deren Nutzung zeitlich durch die Festlegung der voraussichtlichen betriebsgewöhnlichen Nutzungsdauer begrenzt ist, ${ }^{323}$ zu den Anschaffungs- oder Herstellungskosten vermindert, um Abschreibungen anzusetzen. $^{324}$

\subsubsection{Kostenstellenrechnung in deutschen Krankenhäusern:}

\subsubsection{Definition und Aufgaben der Kostenstellenrechnung in deutschen Krankenhäusern:}

Die Einführung der Kostenstellen in deutschen Krankenhäusern ist aufgrund der rechtlichen Verpflichtung obligatorisch und ist grundsätzlich als Vollkostenrechnung zu konzipieren. ${ }^{325}$ Lt. $§ 8 \mathrm{KHBV}$ sind alle Kosten und Leistungen verursachungsgerecht nach Kostenstellen zu erfassen. Sie sind darüber hinaus den

322 Nach dem seit 01. Januar 1996 geltenden $\S 18$ bHG besteht die Möglichkeit, Rationalisierungsinvestitionen über den Pflegesatz zu finanzieren. Als Rationalisierungsinvestitionen wurden jene Investitionen und Maßnahmen angesehen, die geeignet sind, alsbald die Leistung des Krankenhauses kostengünstiger zu erbringen. Vgl. Tanski, J. S. (1997), S.55.

323 Nach $\S 8$ Abs. 1 BPflV sind Abschreibungen auf Anlagegüter (Absetzungen für Abnutzung) nach denselben Grundsätzen zu berücksichtigen, wie sie für dieselben Anlagegüter nach steuerrechtlichen Vorschriften zulässig sind. Darüber hinaus sind Abschreibungstabellen des Bundesfinanzministers und die Abschreibungstabelle im Stichwörterkatalog (Krankenhausrechnungswesen) der Krankenhausgesellschaft, Baden-Württemberg für das Krankenhaus anwendbar. Vgl. Deutsche Krankenhausgesellschaft (1992): Hinweise der DKG zum Rechnungswesen der Krankenhäuser unter besonderer Berücksichtigung der Anpassung des Rechnungswesens der Krankenhäuser in der neuen Bundesländern an die Erfordernisse des Krankenhausfinanzierungsrechts, Düsseldorf, 1992, S. 52- 53.

${ }^{324}$ Vgl. § 5 Abs. 1 KHBV.

325 Nach den Vorschriften der KHBV ist die Kostenstellenrechnung obligatorisch, soweit das Krankenhaus nicht nach $\S 9 \mathrm{KHBV}$ von der Führung einer Kosten- und Leistungsrechnung befreit ist. Vgl. Deutsche Krankenhausgesellschaft (1992), S. 156; 171. §9 KHBV lautet: "Ein Krankenhaus mit bis zu 100 Betten oder mit nur einer bettenführenden Abteilung kann von den Pflichten nach $\S 8$ befreit werden, soweit die mit diesen Pflichten verbundenen Kosten in keinem angemessenen Verhältnis zu dem erreichbaren Nutzen und die in $\$ 8$ Satz 1 genannten Zwecke auf andere Weise erreicht werden können. Über die Befreiung entscheidet auf Antrag 
anfordernden Kostenstellen zuzuordnen, soweit dies für die in Satz 1 genannten Zwecke $^{326}$ erforderlich ist. ${ }^{327}$

Die Aufgaben der Kostenstellen im deutschen Krankenhaus können gesetzlich so differiert werden, dass einerseits die Aufgaben von kostenstellenbezogener Kostenund Wirtschaftlichkeitskontrolle sowie Überwachung kostenstellenbezogener Budgets für den Nachweis der geforderten Kostenbudgets immer als eine Informationsgrundlage gegenüber den Krankenkassen existiert. Diese erlaubt eine Beurteilung der Wirtschaftlichkeit. Andererseits wird im Rahmen der abrechnungstechnischen Aufgaben der Kostenstellen mit der Einführung differenzierter Abteilungsbudgets neben der Überwachung des Kostenbudgets zusätzlich notwendig, ${ }^{328}$ das Krankenhaus in einzelne zu überwachende Abrechnungsbereiche zu untergliedern. Zur Ermittlung der abteilungsbezogenen Budgets müssen alle Kosten über die Kostenstellenrechnung fließen. Damit müssen nicht nur die Gemeinkosten über die Kostenstellenrechnung geleitet werden, sondern alle Kosten einschließlich der Kostenträgereinzelkosten. ${ }^{329}$

Schließlich wird mit der Einführung des Vergütungssystems, wobei die Gesamtkosten entsprechend ihrer Deckung differenziert erfasst werden müssen, eine Vorbereitung für die nachfolgende Kostenträgerrechnung ermöglicht.

\subsubsection{Bildung und Kontierung der Kostenstellen in deutschen Kranken- häusern}

Analog zur Kostenartenrechnung ist auch für die Kostenstellenrechnung durch den deutschen Gesetzgeber in der Anlage 5 der KHBV ein an funktionalen Gesichtspunkten orientierter Kostenstellenrahmen als Mindestgliederungsschema vorgegeben (Abb. 24).

des Krankenhauses die zuständige Landesbehörde; dabei sind einvernehmliche Regelungen mit dem Landespflegesatzausschuss nach $\S 20$ BPflV anzustreben.“

${ }^{326}$ Vgl. § 8 Abs. 1 KHBV: „Das Krankenhaus hat die auf Grund seiner Aufgaben und Struktur erforderlichen Kostenstellen zu bilden. Es sollen, sofern hierfür Kosten und Leistungen anfallen, mindestens die Kostenstellen gebildet werden, die sich aus dem Kostenstellenrahmen der Anlage 5 ergeben."

327 Vgl. $\$ 8$ Abs. 3 KHBV.

328 § 3 Abs. 1 BPflV lautet: „.... Das Budget und die Pflegesätze nach § 10 müssen medizinisch leistungsgerecht sein und einem Krankenhaus bei wirtschaftlicher Betriebsführung ermöglichen, den Versorgungsauftrag zu erfüllen...“ 


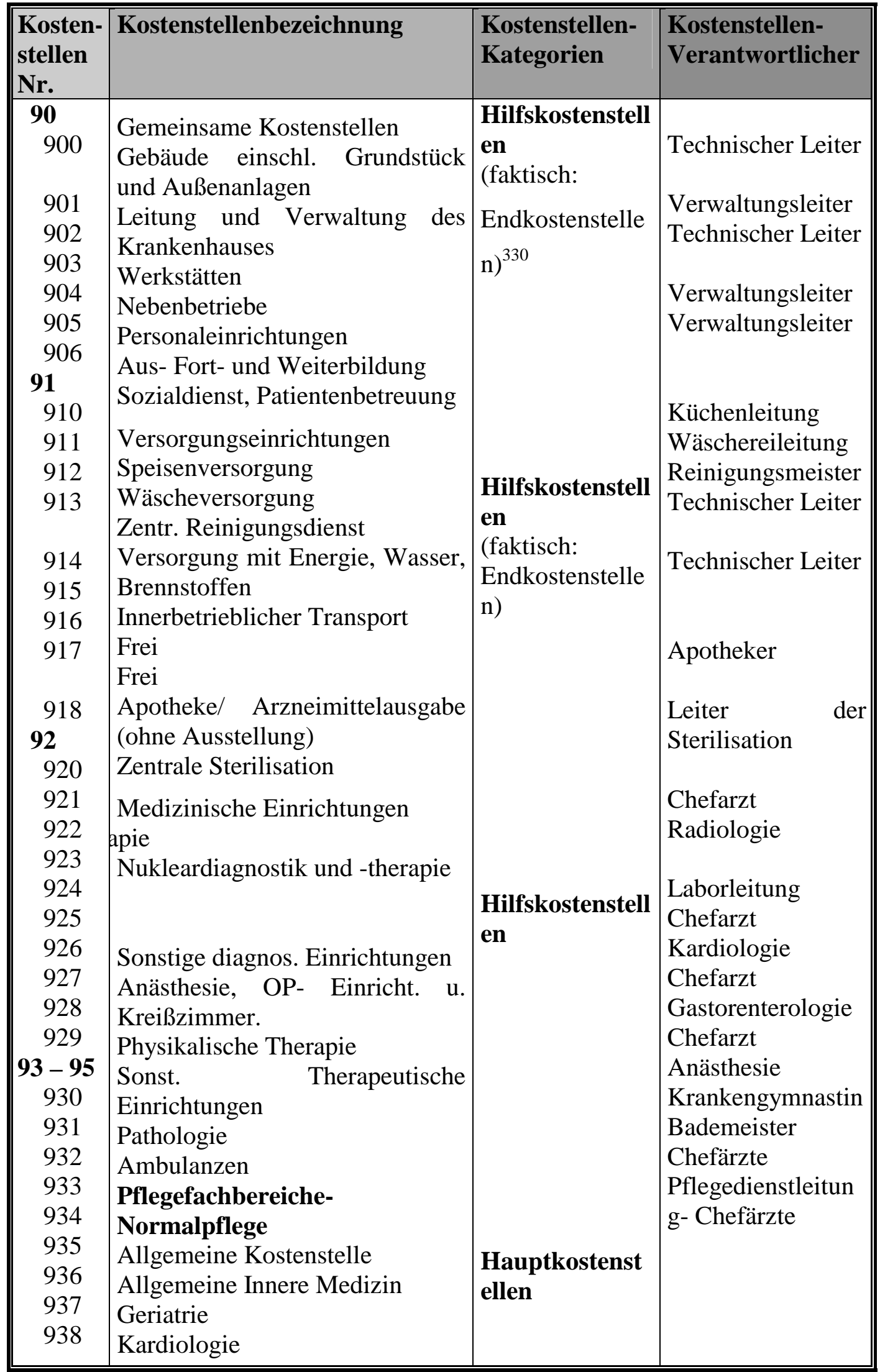

${ }^{329}$ Vgl. Rohrig, R., Schnee, S. (1995), S. 276.
${ }^{330}$ Vgl. 4.2.2.3 


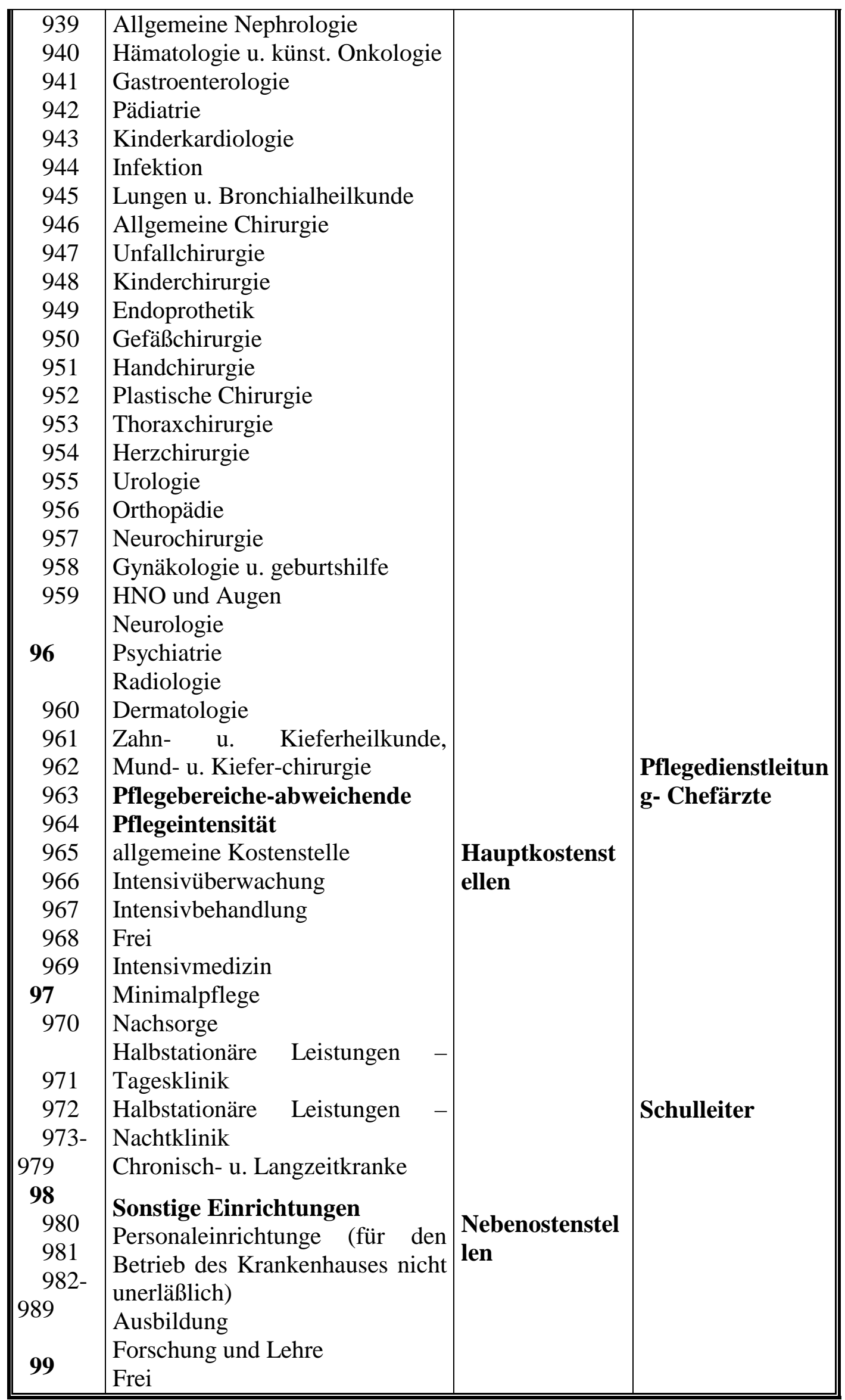




\begin{tabular}{||l|l|l||}
\hline Ausgliederung & Nebenostenstel \\
Ambulanzen & len \\
Hilfs- und Nebenbetriebe & (nach allein \\
Frei & $\begin{array}{l}\text { rechnungs- } \\
\text { technischen } \\
\text { Frei }\end{array}$ & $\begin{array}{l}\text { Gesichts- } \\
\text { punkten zur } \\
\text { Kostenausgliede } \\
\text { rung } \\
\end{array}$ \\
\hline
\end{tabular}

Abb. 24: Kostenstellenrahmen für die Kosten- und Leistungsrechnung nach KHBV. ${ }^{331}$

Dieser Kostenstellenrahmen ist gemäß $\S 8$ Nr. 1 KHBV für alle Krankenhäuser verbindlich, d.h. dass mindestens die im Kostenstellenrahmen aufgeführten Kostenstellen gebildet werden müssen. Da diese Einteilung in Kostenstellengruppen bzw. Kostenstellen relativ grob ist, ist eine beliebig feine Untergliederung von den betreffenden Krankenhäusern selbst unter Beachtung der gängigen Grundsätze (Übersichtlichkeit, rechnungstechnische Gründsätze und Verantwortungsgesichtspunkte) in Abhängigkeit von Betriebsgröße, Leistungsstruktur sowie Aufbau- und Ablauforganisation vorzunehmen. ${ }^{332}$ Beispielsweise kann ein Krankenhaus zum Zweck der Gewährleistung einer leistungsgerechten Nachkalkulation der Entgelte sowie einer wirkungsvollen Kostenkontrolle die Unterkostenstellen 922 Laboratorien in 9221 Hämathologie, 9222 Immunologie und 92223 Mikrobiologie, auch die Unterkostenstellen 941 Chirurgie in 9410 Allgemeine Krankenhaus, 9411 Station I und 9412 Station II . . usw. weiter untergliedern.

Unter Berücksichtigung der Abteilungspflegesätze, die im neuen Entgeltsystem gefördert wurden, sind die Kostenstellen gemäß der in der Betriebsabrechnung zu erfolg innerbetrieblichen Kostenverrechnung hierarchisch zu gliedern. Analog dieser Abrechnungstechnik muß zwischen Vor- und Endkostenstellen unterschieden werden. Innerhalb dieser sind die sogenannten Haupt- und Nebenkostenstellen zu unterscheiden. Alle Kosten des originären Krankenhausbetriebs sind den Hauptkostenstellen zuzuordnen (Basis-, Abteilungspflegesätze,

${ }^{331}$ In Anlehnung an: $\S 8$ Nr. 1, Anlage 5 KHBV und Maltry, H., Strehlau-Schwoll, H. (1997), S. 544. 
Fallpauschalen und Sonderentgelte). Nebenkostenstellen erstellen Leistungen, die mit der eigentlichen Aufgabe des Krankenhauses nicht im unmittelbaren Zusammenhang stehen (z. B. Erlöse aus Personalwohnbereich, Fremdwäscherei).

Gemäß $§ 8 \mathrm{KHBV}$ sind Kosten und Leistungen verursachungsgerecht nach dem Ort ihrer Entstehung zu ermitteln. Wenngleich die Einzelkosten direkt den Kostenträgern zugerechnet werden können, müssen die Gemeinkosten auf Vor- und Endkostenstellen zugeordnet werden. Abb. 25 stellt beispielhaft einen Kostenstellenkonterungskatalog auf Grund des Kostenstellenrahmens der Anlage 5 KHBV dar.

\begin{tabular}{|c|c|c|}
\hline $\begin{array}{l}\text { Konto- } \\
\text { Nr. }\end{array}$ & Konteninhalt & Kostenstellenzuordnung \\
\hline 60- 64 & Personalkosten & $\begin{array}{l}\begin{array}{l}\text { Zuordnung auf } \\
\text { entsprechend dem }\end{array} \\
\begin{array}{l}\text { Kostenstellen } \\
\text { DV-Programm } \\
\text { Personalrechnung } \\
\text { (=vorgelagerte }\end{array} \\
\text { Nebenrechnung). }\end{array}$ \\
\hline 65 & Lebensmittel & 91000 \\
\hline 66 & Medizinischer Bedarf & \\
\hline 6600 & Arzneimittel & Materialrechnung Apotheke \\
\hline 6602 & Blut, $\quad$ Blutkonserven, & Materialrechnung Apotheke \\
\hline 6603 & Blutsatzmittel & Materialrechnung Lager \\
\hline 6604 & $\begin{array}{l}\text { Verbandmittel } \\
\text { Ärztliches und pflegeisches }\end{array}$ & Materialrechnung Lager \\
\hline 6606 & Verbandmittel & Materialrechnung Apotheke/ direkt \\
\hline 6607 & Narkose- und sonst. OP-Bedarf & \\
\hline $\begin{array}{l}6608 \\
6609\end{array}$ & $\begin{array}{l}\text { Bedarf für Röngen- und } \\
\text { Nuklearmedizin } \\
\text { Laborbedarf }\end{array}$ & $\begin{array}{l}\text { Materialrechnung Apotheke/ direkt } \\
920\end{array}$ \\
\hline $\begin{array}{l}6610 \\
6611\end{array}$ & $\begin{array}{l}\text { Untersuchungen u. fremden } \\
\text { Instituten }\end{array}$ & $\begin{array}{l}\text { Materialrechnung Apotheke/ direkt } \\
922\end{array}$ \\
\hline $\begin{array}{l}6612 \\
6613\end{array}$ & $\begin{array}{l}\text { Bedarf für EKG, EEG } \\
\text { Sonographie }\end{array}$ & $\begin{array}{l}\text { Direkt } \\
\text { Direkt } 923\end{array}$ \\
\hline 6616 & Bedarf der physikalischen & Direkt 926 \\
\hline 6617 & $\begin{array}{l}\text { Therapie } \\
\text { Feindesinfektionsmittel }\end{array}$ & $\begin{array}{l}\text { Materialrechnung Apotheke } \\
\text { Direkt } 925\end{array}$ \\
\hline 67 & $\begin{array}{l}\text { Implantate } \\
\text { Kosten für Krankentransporte }\end{array}$ & $\begin{array}{l}\text { Direkt } \\
\text { Direkt }\end{array}$ \\
\hline 68 & Sonstiger $\quad$ Medizinischer & \\
\hline $\begin{array}{l}6800 \\
6801\end{array}$ & Bedarf & 913 \\
\hline
\end{tabular}

332 Vgl. Trill, R. (2000), S.416., und Vgl. Strehlau-Schwoll, H. (1997), S. 543. 


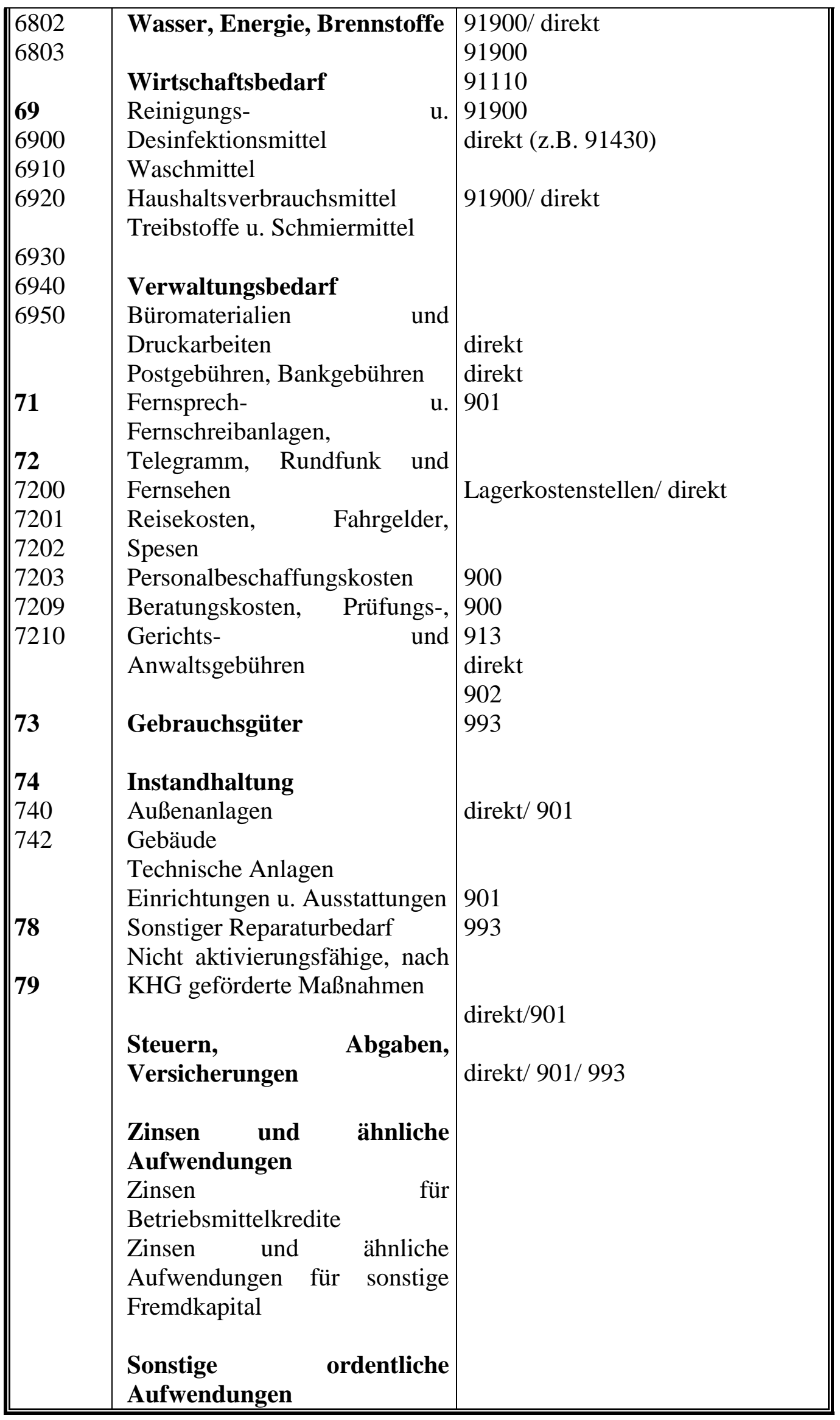




\section{Übrige Aufwendungen}

Abb. 25: Kostenstellenkontierungskatalog. ${ }^{333}$

Die Kostenstellen in den deutschen Krankenhäusern sind so gebildet, dass es in den meisten Fällen möglich ist, die Kosten an einer Kostenstelle direkt zu erfassen. Dazu gehören die Kostenstellengruppen 90 (Gemeinsame Kostenstellen) und 91 (Versorgungseinrichtungen) $\mathrm{zu}$ den Allgemein-Kostenstellen, die für alle Krankenhausteile Leistungen erbringen (Hilfskostenstellen). Beispielsweise existiert neben den Stationen eine allgemeine Kostenstelle, der die Kosten zugeordnet werden, die alle Stationen der Fachabteilung gemeinsam betreffen, die jedoch diesen nicht direkt zugeordnet werden können (z. B. Ärztlicher Dienst, Chefarztsekretärin). Bei den Kostenstellengruppen 92 (Medizinische Institutionen) handelt es sich um Vorkostenstellen, soweit ihre Leistungen stationäre und teilstationäre Patienten betreffen, und um Endkostenstellen, wenn Leistungen für ambulante Patienten erbracht werden. In den Endkostenstellen Kontengruppen 93-96 (Pflegebereiche) werden die eigentlichen Leistungen (Marktleistungen) des Krankenhauses erstellt (Hauptkostenstellen). Und in den Endkostenstellen Kontengruppen 97-98 (sonstige Einrichtungen und Ausgliederungen) werden die Kosten der Leistungen erfaßt, die nicht $\mathrm{zu}$ den eigentlichen Leistungen des Krankenhauses gehören und an andere Leistungsbereiche des Krankenhauses, an die Kostenträger oder an Nebenkostenträger abgegeben werden können (Nebenkostenstellen). Insbesondere ist bei den Ausgliederungen die Kostenstelle Ambulanz hervorzuheben, wobei die Ambulanzselbstkosten ermittelt werden können und dann den aus der Ambulanz erzielten Erlösen gegenübergestellt werden. $^{334}$

333 Quelle: , Hentze, J., Kehres, E. (1995), S. 72-74.

${ }^{334}$ Vgl. Keun, F. (1997), S. 131. 


\subsubsection{Durchführung der Kostenstellenrechnung in deutschen Kranken-}

häusern

Im Rahmen der Durchführung der Kostenstellenrechnung sollen die Kosten der Vorkostenstellen den leistungsempfangenden Endkostenstellen durch die Auswahl geeigneter Bezugsgrößen möglichst verursachungsgerecht zugeordnet werden.

Eine spezifische Besonderheit auf Grund des Vergütungssystems kommt in deutschen Krankenhäusern vor, ${ }^{335}$ da die gemeinsamen Kostenstellen und Versorgungseinrichtungen (Kontengruppen 90-91) keine Vorkostenstellen stellen, obwohl sie in einer leistungsorientierten Betrachtung lediglich Vorkostenstellen sind. Sie werden durch die Existenz der Entgeltkomponente „Basispflegesatz als künstlicher Kostenträger“ faktisch zu Endkostenstellen, auf den die Kosten des Basisbereichs zu verteilen sind. ${ }^{336}$

Die Kostenstellen Medizinisch-Institutionen „Kontengruppe 92“ sind Hilfskostenstellen, die gleichzeitig Vorkostenstellen darstellen. Eine Ausnahme ist die Kostenstelle „Ambulanz“, die eine Endkostenstellen darstellt.

Wenn die Kostenstellen Leistungen für einzelne Hauptkostenstellen (stationäre Patienten) erbracht haben (z. B. Computertomographie für eine bettenführende Abteilung), werden die Kosten als innerbetriebliche Leistung den anfordernden Abteilungen zugerechnet. Die Zurechnung von innerbetrieblichen Leistungen ist zur Ermittlung der Abteilungspflegesätze bzw. nach den gesetzlichen Vorschriften erforderlich. ${ }^{337}$

Haben die Kostenstellen hingegen Leistungen für ambulante Patienten erbracht, werden diese Leistungen als Marktleistungen zugerechnet, deren Kosten durch die Erlöse im Form von Gebühren gemäß Gebührenordnung der Ärzte (GOÄ) erstattet werden. $^{338}$

\footnotetext{
${ }^{335}$ Vgl. 2.2.3.2.

336 Als künstlicher Kostenträger findet die Bezugsgröße „Berechnungstage“ Verwendung. Die dem Basispflegesatz zuzuordnenden Kostenarten sind dabei in den Teilen K1-K3 der LKA in Anhang $3 \S 17$ Abs. 4 BPflV für Zwecke der Pflegesatzverhandlung und des Krankenhausvergleichs abschließend festgelegt. Vgl. Maltry, H., Strehlau-Schwoll, H. (1997), S. 546.

${ }^{337}$ Vgl. § 8 Abs. 3 KHBV.

338 Vgl. Arnold, M. (1988): Möglichkeiten der Verzahnung des ambulanten und stationären Sektors, in: das Krankenhaus, 3/1988, S. 98- 103, auch Müller, H. (1988): Preisrecht und
} 
Mit dieser differenzierten Zurechnung der innerbetrieblichen sowie ambulanten Leistungen (getrennte Mengen- und Wertkomponente) ${ }^{339}$ ist neben der verursachungsgerechten Verrechnung der Kosten auf die leistungsempfangenden Kostenstellen eine effektive Kosten- und Wirtschaftlichkeitskontrolle durchgeführt worden. Somit werden bei der bettenführenden Abteilung nur die Anzahl der angeforderten Leistungen (Anforderungswirtschaftlichkeit) und bei den Medizinischen Institutionen nur die Kosten der Leistungserbringung (Produktionswirtschaftlichkeit) erfasst. ${ }^{340}$

Die Katalogisierung und Bewertung der Leistungen der Medizinischen Institutionen ist zum großen Teil nach den Leistungskatalogen der GOÄ zu ermitteln, die sowohl die Leistungsarten als auch deren Bewertung in Form von Punkten erfasst. Durch die Anwendung dieser punktbewerteten Leistungskataloge wird es ermöglichen, Äquivalenzziffer (Relation der Kosten und die Leistungen untereinander) zu ermitteln. ${ }^{341}$ Für Leistungen, bei denen keine bewerteten Leistungskataloge vorliegen (z. B. Anästhesie-, Kreißsaal- und OP-Leistungen) müssen andere Faktoren als Verrechnungsgrundlagen eingesetzt werden(dies könnten Zeitfaktoren sein, z.B. Schnitt-Naht-Zeit bei OP-Leistungen). ${ }^{342}$

Nach der Verrechnung der Leistungen der Medizinischen Institutionen sind die Pflegesätze für die bettenführenden Abteilungen der Kostenstellen 93-96 (Pflegebereiche - Normalpflege und Pflegebereiche - abweichende Pflegeintensität) zu ermitteln. Hier müssen die Kosten für die „,anderen Bereichen“ des Krankenhauses, die etwa Ambulanzen, vor- und nachstationäre Behandlung oder wahlärztliche Leistungen umfassen, im Rahmen der Fallpauschalen und Sonderentgelte als künstliche Kostenstellen in die Kostenstellen 98 ausgegliedert werden.

Bei der Kostenstelle 97 „Sonstige Einrichtungen“, die Kosten für nicht zum eigentlichen Krankenhausbetrieb gehörende Leistungen erfasst, und Kostenstelle 98

Gebührenwesen, in: Eichhorn, S. (Hrsg.), Handbuch Krankenhausrechnungswesen Grundlagen- Verfahren- Anwendungen, 2. Aufl., Wiesbaden, 1988, S. 621-624.

339 Die Grundlage der innerbetrieblichen Leistungsverrechnung ist die Leistungsrechnung, in der die Anzahl und der Zeitpunkt der Leistungsabgabe an einzelne Kostenstellen angefordert wurde. Vgl. Abschnitt 4.1.2.3.

${ }^{340}$ Vgl. Hentze, J., Kehres, E. (1995), S. 75.

${ }^{341}$ Vgl. ebenda, S. 110. 
„Ausgliederung“, die keine Leistungsbereiche, sondern auf Grund von rechnungstechnischen Funktionen künstliche Kostenstellen sind, müssen die erbrachten Leistungen und die dafür entstandenen Kosten separat gegenübergestellt werden. ${ }^{343}$

\subsubsection{Kostenträgerrechnung in deutschen Krankenhäusern}

\subsubsection{Definition und Aufgabe der Kostenträgerrechnung in deutschen Krankenhäusern:}

In der Terminologie des deutschen Krankenhausfinanzierungsrechts werden unter dem Begriff „Kostenträger“ üblicherweise diejenigen verstanden, die zur Zahlung der stationären und ambulanten Behandlungskosten verpflichtet sind (gesetzliche Krankenkassen, private Krankenversicherungen oder Selbstzahler). ${ }^{344}$

Aus Sicht der Kostenrechnung sind die Kostenträger im Sinne des Krankenhausfinanzierungsgesetzes vor allem allgemeine Krankenhausleistungen, Wahlleistung und ambulante Behandlung. Die Kostenträgerrechnung im deutschen Krankenhaus ist gesetzlich nicht vorgeschrieben, so daß für ihre Einführung keine terminlichen Vorgaben zu beachten sind. Bis zum Gesundheitsstrukturgesetz (GSG) 1993 und der Novellierung der Bundespflegesatzverordnung (BPflV) 1995 war es nicht notwendig, über eine Kostenträgerrechnung zu verfügen.

Eine Kostenträgerrechnung war dann jedoch sinnvoll, aber nicht gesetzlich vorgeschrieben, wenn krankenhausindividuelle Sonderentgelte vereinbart worden sind.

Einerseits wird empfohlen, Überlegungen zur Kostenträgerrechnung erst nach dem vollständigen Aufbau der Kostenarten- und Kostenstellenrechnung vorzunehmen, da der deutsche Gesetzgeber im $\S 8$ KHBV und den Bestimmungen des $\S 17$ Abs. 2 Satz 2 KHG konkretisiert hat, dass die Kosten der Krankenhausleistungen auf der Grundlage der kaufmännischen Buchführung und Kosten- und Leistungsrechnung zu ermitteln sind.

Andererseits, obwohl der Gesetzgeber in der KHBV nur die Kostenarten- und Kostenstellenrechnung vorschreibt, ist zur Erfüllung der gesetzlich festgelegten

342 Vgl. Rippel, W. H., Posininsky, R. (1990): Praktische Anwendung der Kostenarten- und Kostenstellenrechnung, in: das Krankenhaus, 11/ 1990, S. 489- 490.

${ }^{343}$ Vgl. Hentze, J., Kehres, E. (1995), S. 63-64. 
Aufgaben der Kostenrechnung (Betriebsteuerung und Wirtschaftlichkeitskontrolle), zur Herstellung von Kostentransparenz und zur Ermittlung der Selbstkosten jedoch eine Kostenträgerrechnung unabdingbar. Einige Bedenken ${ }^{345}$ äußern, dass die Anforderungen an eine differenzierte Kostenverteilung auf Kostenträger im deutschen Krankenhaus nicht sehr hoch sind, weil die Kosten der stationären Behandlung im Wesentlichen durch die gesetzlichen Krankenkassen und die privaten Krankenversicherungen auf der Grundlage des Solidaritätsprinzips getragen werden. Damit wird bei der Kostenverteilung das Gesetz der großen Zahl wirksam, d. h. es kommt weniger darauf an, inwieweit die Kosten für den einzelnen Patienten sachgerecht ermittelt wurden, sondern vielmehr darauf, ob die insgesamt von einer Krankenkasse zu tragenden Kosten in einem angemessenen Verhältnis zu den erbrachten Leistungen stehen.

Es wurde bereits vor Einführung des differenzierten Entgeltsystems über die Einführung einer Kostenträgerrechnung diskutiert, wobei schon die Wahl eines geeigneten Kostenträgers unter Berücksichtigung von traditionellen, industriell geprägten Vorgaben Schwierigkeiten bereitet. $^{346}$

Als Aufgaben der Kostenträgerrechnung im deutschen Krankenhaus werden neben der Ermittlung der Kostenträgerkosten die Aufgaben von der Preisermittlung, Ermittlung der Wirtschaftlichkeit und Planung, Steuerung und Analyse des Leistungsprogramms genannt. Für die Preisermittlung und -beurteilung ist immer das gültige Entgeltsystem zu berücksichtigen. ${ }^{347}$ Durch das deutsche Entgeltsystem sollen bei den Fallpauschalen und Sonderentgelten keine Kosten mehr erstattet, sondern Leistungen vergütet werden. Deswegen wird insbesondere ein PreisLeistungs-Vergleich zu einer wichtigen Aufgabe der Kostenträgerrechnung. Ein Preissystem kann nur unter den Voraussetzungen zum Tragen kommen, wenn eine Steuerung der Leistungsmenge durch das Krankenhaus möglich ist, wenn die Vergütungen nicht in Anlehnung an die individuellen Selbstkosten des jeweiligen

\footnotetext{
344 Vgl. Deutsche Krankenhausgesellschaft (1992). S. 171.

345 Vgl. Kehres, E. (1990): Kosten und Kostendeckung der ambulanten Behandlung im Krankenhaus, Dissertation an der Uni. Braunschweig, 1990, S. 105- 106.

346 Vgl. Hübner, H. (1980): Kostenrechnung im Krankenhaus, Grundlagen, Wirtschaftlichkeitsanalyse, Betriebsvergleich, 2. Aufl., Stuttgart, Berlin, Köln, Mainz, 1980, S. 62.

347 Vgl. Brayer, F. (1991), S. 62.
} 
Krankenhauses ermittelt werden, und spätestens ab 1998, seitdem das Krankenhaus Gewinne erwirtschaften kann. ${ }^{348}$

\subsubsection{Bildung von Kostenträgern in den deutschen Krankenhäusern}

Für die Bildung von Kostenträgern in der Krankenhaus-Kostenrechnung ist der Pflegetag bzw. der Berechnungstag als Kostenträger gesetzlich nach $§ 18$ BPflV vorgegeben. ${ }^{349}$ Aber da der Pflegetag für laufende Planungs- und Kontrollzwecke nicht geeignet ist, und im Sinne einer Kostenrechnung nicht als Einzelleistung anzusehen ist, da in den deutschen Krankenhäusern eine Reihe unterschiedlicher Leistungen entstehen, können in der Praxis vielfältige Leistungseinheiten als Kostenträger definiert werden (z. B. Leistungen des Versorgungsbereichs, Diagnostik- und Therapieleistungen des Funktionsbereiches, pflegerische und ärztliche Leistungen im Pflegebereich und Leistungen des Verwaltungsbereichs).

Die Versorgungsleistung stellt die Gesamtleistung des Krankenhauses als Aggregation von Einzelleistungen für einen bestimmten Personenkreis dar. Wird die Versorgung auf einen Zeitraum bezogen, ergibt sich im Rahmen der Leistungsentgeltung die Notwendigkeit zu einer Flexibilisierung des Krankenhausbudgets nach der Zahl der erbrachten Pflegetage, der Zahl der Zusammensetzung der Krankheitsfälle oder des Umfangs und der Struktur der Bevölkerung. ${ }^{350}$

Einzelleistungen ergeben sich aus der Kombination von Produktionsfaktoren und stellen den primären Input für die Versorgung des einzelnen Patienten dar. Durch diese Leistungen (Sekundärleistung) wird die Primärleistung (die Statusveränderung des Patienten) herbeigeführt. ${ }^{351}$ Die Marktleistung des Krankenhauses ist eine Zusammenfassung heterogener Einzelleistungen an Patienten und nicht die Herstellung des Pflegetages. Als alternativer Kostenträger wurde der Patient als Empfänger der Primärleistung definiert. ${ }^{352}$ In diesem Fall sind klassifizierte Pati-

348 Vgl. Regler, K. (1994), S. 4, und vgl. Keun, F. (1997), S.160.

${ }^{349}$ Vgl. § 18 BPflV.

${ }^{350}$ Vgl. Preuß, O. (1996), S. 65- 66.

351 Vgl. 2.1.5.

352 Teilweise geäußerte ethische Bedenken gegen eine Verwendung von Patienten als Kostenträger kann mit dem Argument begegnet werden, dass ein solches Kostenrechnungssystem nicht gleichzeitig ein inhumanes Krankenhauswesen impliziert. Vielmehr ist ein derartiges System notwendige Voraussetzung zur Aufrechterhaltung des hohen Standards des Gesundheitswesens 
entengruppen/Fallgruppen zu verwenden. Klassifizierungskriterien können nach der Diagnose (Konzept der Diagnosis Related Groups (DRG)) oder der Behandlungsmethode (Konzept der Patient Management Categories (PMC)) praktiziert werden. In Anlage $1 \mathrm{zu} \S 11$ Abs. 1 BPflV erfolgt die inhaltliche Definition der Fallpauschalen durch eine Kombination von Kennziffern der International Classification of DiseasesRevision 9 (ICD-9) und der International Classification of Procedures in Medicine (ICPM).

Auch die verschiedenen Entgeltformen und deren Kombinationsmöglichkeiten bezogen auf den einzelnen Kostenträger führen zur Bestimmung der Kostenträger. Entsprechend regeln die $\S \S 10$ bis 13 BPflV die Vergütung stationärer Leistungen und definieren dementsprechend die Kalkulationsobjekte im Sinne der Kostenträgerrechnung. Sie schreiben solche Kostenträger (wie z. B. tagesgleiche Pflegesätzenach $\S 13 \mathrm{BPflV},{ }^{353}$ und Sonderentgelte und Fallpauschalen nach $\S 11$ BPflV) vor. ${ }^{354}$ Andere Formen der Krankenhausbehandlung gemäß SGB V (§§ 39 Abs. 1 und $73,115 \mathrm{a}$ und $115 \mathrm{~b}$ ) neben stationäre Behandlung sind teilstationäre Behandlung, vor- und nachstationärer Behandlung und ambulantes Operieren. $\mathrm{Zu}$ den Kostenträgern gehören aber auch die Leistungen, deren Kosten nach der BPfIV a. F. in Teil K3 des Kosten- und Leistungsnachweises ausgegliedert wurden ${ }^{355}$ (ambulante Behandlung durch das Krankenhaus als Institut und ambulante Behandlung durch Ärzte des Krankenhauses).

in Deutschland und daher mit ethischen Vorstellungen absolut kompatibel. Vgl. Tscheulin, D. K., Hemig, B. (1994), S. 502.

353 Mit der neuen Bundespflegesatzverordnung (BPflV) wird der allgemeine Pflegesatz durch Abteilungspflegesätze (wobei die ärztlichen und pflegerischen Leistungen des Krankenhauses differenziert nach Fachabteilungen in unterschiedlicher Höhe vergütet werden) und Basispflegesatz (wobei die übrigen Leistungen des Krankenhauses, insbesondere Unterkunft, Verpflegung und Verwaltungsleistungen vergütet werden) abgelöst. Dementsprechend setzt sich die Leistungsvergütung für jeden Patienten aus zwei Komponenten zusammen: dem Basispflegesatz und dem Abteilungspflegesatz. Vgl. Abschnitt 2.2.3.2.

354 Die Sonderentgelte beziehen sich auf bestimmte Kostenarten. Das bedeutet, dass die leistungsbezogene Vergütung bei den Patienten, bei denen sonderentgeltfähige Leistungen erbracht werden, künftig aus drei Komponenten (Basispflegesatz, Abteilungspflegesatz und Sonderentgelt) besteht. Die Fallpauschale wurde mit $\S 12$ GSG eingeführt. Sie tritt für bestimmte Patienten bzw. Patientenkategorien an die Stelle von Basispflegesatz, Abteilungspflegesatz und ggf. Sonderentgelt. Vgl. Abschnitt 2.2.3.2.

355 Vgl. Bölke, G. (1987), S. 216- 218. 
In Orientierung an der Art der Vergütung bzw. Kostenerstattung ergeben sich verschiedene Kalkulationsobjekte der Kosten- und Leistungs/Erlösrechnung, die in Tab. 12 dargestellt werden.

\begin{tabular}{|c|c|}
\hline Kalkulationsobjekte & Vergütung bzw. Kostendeckung \\
\hline $\begin{array}{l}\text { Allgemeine } \\
\text { Kranken- } \\
\text { Hausleistungen. }\end{array}$ & $\begin{array}{ll}- & \text { Fallpauschalen (Preis) } \\
- & \text { Sonderentgelte (Preis) } \\
\text { ( nach } \$ 11 \text { BPflV) } \\
\text { - } & \text { Abteilungspflegesätze } \\
- & \text { Basispflegesatz } \\
\text { (nach } \S 13 & \text { i.V.m. } \S 11 \\
\text { BPflV) } & \end{array}$ \\
\hline $\begin{array}{l}\text { Teilstationäre } \\
\text { Krankenhausleistungen: }\end{array}$ & $\begin{array}{l}-\quad \text { nach } \S 112 \text { Abs. } 2 \text { SGBV mit } \\
\text { - } \quad \text { Abteilungspflegesätze } \\
-\quad \text { Basispflegesatz } \\
\text { BPflV) } \\
\text { (nach } § 13 \text { Abs.1 u. } 4\end{array}$ \\
\hline $\begin{array}{l}\text { Vor- und } \\
\text { Behandlung: }\end{array}$ & $\begin{array}{r}\text { - } \quad \text { Abteilungspflegesatz, anteilig } \\
\text { (nach } \S 115 \text { a SGBV) }\end{array}$ \\
\hline $\begin{array}{l}\text { Ambulante ärztliche Leistungen des } \\
\text { Krankenhauses: } \\
\text { - ambulante Operationen } \\
\text { - der Ärzte des Krankenhauses: }\end{array}$ & $\begin{array}{ll}-\quad & \text { nach jeweils geltender Gebühren- } \\
\text { ordnung (Preis) } \\
\text { - } \quad \text { Kostenerstattung durch die Ärzte }\end{array}$ \\
\hline Wahlärztliche Leistungen: & $\begin{array}{l}\text { - Kostenerstattung durch die Ärzte: } \\
\text { Gebühren lt. Gebührenordnung } \\
\text { bzw. Nutzungsentgelte der Ärzte lt. } \\
\text { Dienstvertrag. }\end{array}$ \\
\hline $\begin{array}{l}\text { Gesondert } \\
\text { Unterkunft: }\end{array}$ & - Tagesbezogenes Leistungsentgelt \\
\hline
\end{tabular}

Tab 12: Kalkulationsobjekte der Kosten- und Leistungs/Erlösrechnung und deren Vergütungsformen. ${ }^{356}$

\subsubsection{Durchführung der Kostenträgerstückrechnung in deutschen Kran- kenhäusern:}

Obwohl keine gesetzlichen Vorschriften zur Durchführung der Kostenträgerrechnung in deutschen Krankenhäusern existieren, werden aber die Selbstkosten der jeweiligen Kostenträger bei der Kalkulation der Fallpauschalen und Sonderentgelte 
ermittelt. Mit der Gegenüberstellung von diesen Selbstkosten und den von außen vorgegebenen Preisen wird der Erfolg je Kostenträger ermittelt. Die Ermittlung der Kosten eines Kostenträgers im deutschen Krankenhaus erfolgt demnach zum einen durch die Anwendung der Verrechnungssatzkalkulation gemäß der Inanspruchnahme von spezifischen Krankenhausleistungen (Fallpauschalen und Sonderentgelte), wobei die Kosten einzelner Kostenstellen proportional zu deren Leistungsvolumen verrechnet werden, und den kostenstellenbezogenen Kosten, die kostenstellenbezogenen Leistungen gegenübergestellt werden, ${ }^{357}$ und zum anderen durch die Anwendung der Divisionskalkulation auf der Basis der Berechnungstage eines Krankenhauses bzw. einer Abteilung (Basispflegesatz und Abteilungspflegesätze). ${ }^{358}$

Die Kostenträgerrechnung wird bei der Mehrzahl der deutschen Krankenhäuser mit der traditionellen Vollkostenrechnung, d. h. mit Elementen einer Divisions- und einer differenzierten Zuschlagkalkulation durchgeführt, ${ }^{359}$ da die Ermittlung der Selbstkosten und der Vollkosten für ambulante Leistungen vorgeschrieben, und mit der Kostenausgliederung eine Kalkulation von Fallpauschalen und Sonderentgelten auf Basis der Vollkosten zum 01. Januar 2000 Pflicht ist. ${ }^{360}$ Die Durchführung der Kostenträgerrechnung auf Teilkostenbasis hat für die Kalkulation von Fallpauschalen und Sonderentgelten in der Praxis wenig Bedeutung, weil zum einen durch den geringen Anteil der variablen Kosten der Stückdeckungsbeitrag wenig Aussagekraft besitzt und zum anderen das Krankenhaus aus kurzfristiger Sicht die Gestaltung des Leistungsprogramms nicht beeinflussen kann. Es empfehlt sich jedoch, im Sinne der Erfüllung einer Mehrfachzwecksetzung (z. B.

${ }^{356}$ In Anlehnung an: Schmidt- Rettig, B.(1995), S. 139, bzw. Heintze, J., Kehres, E. (1995), S. 25.

357 Vgl. Hummel, S., Männel, W. (1986), S. 301.

${ }^{358}$ Bei den Kalkulationen der Kosten eines Berechnungstags im Basisbereich oder einer der bettenführenden Abteilungen sind letztere zuerst zu ermitteln, da sie erst nach der Ausgliederung der Kosten in gerade diesem Bereich für die Erbringung von durch Fallpauschalen und Sonderentgelte vergüteten Leistungen entstanden sind. Vgl. Maltry, H., Strehlau-Schwoll, H. (1997), S. 548.

359 Vgl. Klask, J., Schelzer, A. (1997): Fallkostenkalkulation der Therapien Lonsillektomie und Septimplastik, Gesamtkosten unter Berücksichtigung typischer Komplikation, in: Deutschland Krankenhaus, 2/ 1997, S. 60; Vgl. Abschnitt 4.1.3.

${ }^{360}$ Vgl. Tuschen, K. H. (1997): 5. Änderungsverordnung zur BPflV, Stand: Regierungsbeschluß, in: Krankenhausumschau, 8/1997, S. 279. 
Unternehmenssteuerung oder Preisbildung) parallel dazu eine Grenzkostenrechnung (Verfahren der Parallel- oder Doppelkalkulation) durchzuführen. ${ }^{361}$

Im Rahmen des Verfahrens der Kostenträgerstückrechnung in den deutschen Krankenhäusern kommt einerseits das Verfahren der Divisionskalkulation zur Anwendung in Fällen homogener Kostenverursachung einer Kostenstelle für die Ermittlung der Verrechnungssätze. Andererseits kann bei heterogener Kostenverursachung unter Umständen eine Äquivalenzziffernrechnung angewendet werden, um verschiede Leistungen einer Kostenstelle vergleichbar zu machen (z. B. über die Punktzahl der GOÄ). ${ }^{362}$

Zur Ermittlung der Kostenträgerrechnung muss ein gewähltes Verfahren sich aber für die unterschiedlichen Entgeltbasis ergänzen. In der Praxis der deutschen Krankenhäuser sind Kostenträger seit 01.01.1996 in Form von Fallpauschale, Sonderentgelt, Basispflegesatz und Abteilungspflegesätze ${ }^{363}$ ermittelt worden.

\subsection{Kalkulation von Fallpauschalen}

Die Kalkulation von Fallpauschalen beinhaltet die Ermittlung aller pflegesatzrelevanten Kostenbeträge für das gesamte Leistungsbündel eines festgelegten Behandlungsfalls. Die Voraussetzung für die Güte der Kalkulationsergebnisse ist insbesondere eine differenzierte Fallgruppenbildung, die sich sowohl an der Diagnose als auch an dem Therapieverfahren orientiert. ${ }^{364}$ Das heißt, Fallpauschalen sind vor allem für häufige und diagnostisch/therapeutisch eindeutig abgrenzbare Leistungen vorgesehen. Die Leistungsabgrenzung erfolgt durch die fallgruppenspezifische Festlegung des Leistungsspektrums der direkten Patientenversorgung. Damit sind die Leistungen der medizinischen Institutionen, insbesondere des OP-

\footnotetext{
361 Vgl. Tscheulin, D. K., Hemig, B. (1994), S. 503-504, und Maltry, H., Strehlau-Schwoll, H. (1997), S. 549.

362 Als Verrechnungssätze sind etwa „DM je OP-Minute“ für den Funktionsdienst OP (bei gegebenen Personalsätzen nach Art und Menge und gegebenen Kosten) oder „DM je GOÄ-Punkt“ für Leistungen der Röntgendiagnostik (bei einer die Gesamtleistung der Kostenstelle wiedergebenden Punktzahl nach GOÄ und gegebenen Kosten) vorstellbar. Vgl. Maltry, H., Strehlau-Schwoll, H. (1997), S. 548-549.

${ }^{363} \mathrm{Vgl}$. Abschnitt 2.2.3.2.
} 
Bereichs, sowie die Leistungen der Stationen (Normalpflege und Intensivstationen) angesprochen. ${ }^{365}$

Die Behandlungsfälle des Fallpauschalenkataloges (ebenfalls des Sonderentgeltkataloges) sind bundeseinheitlich mit Punktezahlen gewichtet, die die unterschiedliche Kostenintensität der Leistungen untereinander festlegen. Die Bestimmung der Höhe der Fallpauschalenentgelte richtet sich dann nach dem landesweit zwischen Krankenhäusern und Krankenkassen vereinbarten Punktswert. ${ }^{366}$ Für die Ermittlung von Fallpauschalen soll sich der kalkulatorische Ansatz auf die Leistungen der direkten Patientenversorgung konzentrieren. Basisleistungen werden über den Basispflegesatz unter Berücksichtigung der fallspezifischen durchschnittlichen Verweildauer in die Fallpauschale eingerechnet. Der kalkulatorische Ansatz ist von der Methode her eine Verrechnungssatzkalkulation (Bezugsgrößenkalkulation), bei der in den kalkulatorischen Teilschritten mit der Divisionskalkulation, insbesondere der Äquivalenzziffernrechnung, gearbeitet wird. ${ }^{367}$ Die Methodik zur Kalkulation von Fallpauschalen ist in Abb. 25 dargestellt.

\footnotetext{
364 Die Behandlungsfälle sind in der Anlage 1 als Bestandteil der BPflV katalogisiert. Der derzeitige Katalog umfasst rund 40 Fallpauschalen, die ausnahmslos operative Leistungen betreffen. Vgl. $\S 11$ Abs. 1 BPflV und Preuß, O. (1996), S. 69.

365 Vgl. Heintze, J., Kehres, E. (1995), S. 138.

366 Vgl. § 16 Abs. 1 BPflV.

${ }^{367}$ Vgl. Heintze, J., Kehres, E. (1995), S. 139.
} 


\section{Stationsleistungen}

Normalstation:

Ärztlicher Dienst:

Pflegedienst:

Pflegetag $\times$ Tagessatz (Arzt)

Intensivstation:

Ärztlicher Dienst ${ }^{370}$ :

Pflegeminuten $\left(\right.$ nach PPR) ${ }^{368} \times$ Minutensatz $^{369}$

Pflegedienst:

Arztminuten $\times$ Minutensatz

Pflegeminuten $\times$ Minutensatz

\section{OP- Leistungen}

Ärztlicher Dienst:

Minutensatz

Funktionsdienst: ${ }^{371}$

Pflegekraft $\times$ Minutensatz.

Gleichzeitigkeitsfaktor $\times$ Bruttozeitaufwand je Arzt $\times$

Ärztlicher Dienst der Anästhesie: Arztminuten-Anästhesiezeit $\times$ Minutensatz

Funktionsdienst der Anästhesie: Pflegeminuten-Anästhesiezeit $\times$ Minutensatz

Medizinisch- technischer Dienst: Zeiten des Medizinisch- technischen

Dienstes $\times$ Minutensatz

\section{Leistungen des Untersuchungs- und Behandlungsbereichs}

Patientenbezogener Zeitaufwand für Sekundärleistungen $\times$ Minutensatz der Betriebsstellen des Untersuchungs- und Behandlungsbereichs

Oder:

Verrechnung der Untersuchungs- und Behandlungsleistungen nach Gebührenwerken

\section{Basisleistungen}

Pflegetage $\times$ Personalkostenanteil des Basispflegesatzes

\section{Sachmitteleinsatz}

\begin{tabular}{|lc}
\hline A Artikel: & Verbrauchsmenge $\times$ Listenpreis \\
$\begin{array}{l}\text { B-Artikel: } \\
\text { technischem }\end{array}$ & Der Verbrauch an medizinischem Bedarf, medizinisch- \\
Pflegetage bezogen & Bedarf und der sonstige Verbrauch wird auf die
\end{tabular}

Abb. 26: Das Kalkulationsschema für die Ermittlung einer Fallpauschale. ${ }^{372}$

${ }^{368}$ Die Ermittlung der Kosten des Pflegedienstes auf Normalstation erfolgt auf Grundlage der Kategorisierung des Patienten nach der Pflege- Personalregelung.

369 Die Minutenwerte der einzelnen Kategorien ergeben durch Multiplikation mit einem festgelegten Geldsatz (Minutensatz) die täglichen Kosten für die Pflege des Patienten. Vgl. Baugut, G., Schmitz, R., (1994), S. 172.

${ }^{370}$ Die Umrechnung auf Intensivleistungen des ärztlichen und pflegerischen Bereichs (Überwachungs-, Behandlungs- und Beatmungsleistung) erfolgt mit Hilfe von Äquivalenzziffern. Vgl. Baugut, G., Schmitz, R., (1994), S. 173.

371 Funktionsdienst umfaßt die Leistungen des Labors oder der Röntgendiagnostik und anderer Kostenstellen des Untersuchungs- und Behandlungsbereichs medizinischer Institutionen des eigenen Krankenhauses. 
Im Folgendem werden anhand einer Fallpauschale, die sich aus einer Mengen- und einer Bewertungskomponente zusammensetzt, die Mengenkomponenten, die Kostenkomponenten sowie die Verknüpfung beider mit dem Verfahren der Bezugsgrößenkalkulation dargestellt.

Es handelt sich um die Fallkategorie Nr. 17/02 „Schenkelhalsfraktur geschlossen (TEP)“: ${ }^{373}$

- Diagnose: Schenkelhalsfraktur geschlossen,

- Therapie: Einbau einer Hüftkopf-/Schaftprothese:19 Tage Verweildauer, davon 18 Tage Normalstation Chirurgie und 1 Tag Intensivstation.

A. Stationsleistungen: Sie umfassen den ärztlichen und pflegerischen Personaleinsatz auf der Normal- und Intensivstation:

a. Normalstation:

i. Arztdienst (AD):

- Mengenkomponente: 18 Tage ${ }^{374}$

- $\quad$ Ermittlung des Kostensatzes:

Personalkosten AD/Station p.a. $\quad$ 864.247 DM

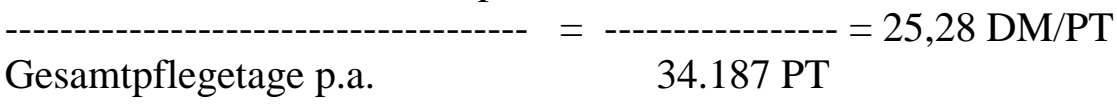

Ermittlung der Fallkosten: $18 \mathrm{PT} \times 25,28 \mathrm{DM} / \mathrm{PT}=\underline{455,04 \mathrm{DM}}$

ii. Pflegedienst (PD):

- Mengenkomponente: $3.446 \mathrm{Min}^{: 375}$

372 Quelle: o. V. Fallpauschalen- die Empfehlungen der Institute, in: F\&W, 10 Jahrgang, 1993, S.401.

373 In Anlehnung an des Berichts zum Forschungsprojekt „Kalkulation von Fallpauschalen und Sonderentgelten gemäß BPflV 95“ im Auftrag des Bundesministeriums für Gesundheit. Vgl. Bundesministerium für Gesundheit (1995 a), Teilbericht III, S. 22-26.

${ }^{374}$ Zur Beschreibung der zeitlichen Bindung des Ärztlichen Dienstes auf der Station wird bei den operativen Fallkosten vereinfachend lediglich die Verweildauer - differenziert nach Fachabteilungen - herangezogen. Statt der vereinfachenden Schlüsselung besteht die Möglichkeit einer differenzierten Erfassung der zeitlichen Bindung des Arztdienstes anhand der Verrichtung gegliedert nach Tätigkeitsarten (Visite, Diagnose, Therapie, Dokumentation ). Es ist sinnvoll, den ärztlichen Personaleinsatz nach Qualifikationsstufen (AIPler, Assistenzarzt, Oberarzt sowie Chefarzt) zu differenzierten, was eine Bewertung mit differenzierten Kostensätzen zur Folge hätte.

375 Die zeitliche Bindung des Pflegepersonals auf der Normalstation ist anhand der Verweildauer differenziert nach Fachabteilungen und gestuft nach Pflegekategorien in der Pflegepersonalregelung (PPR) beschrieben. 
Für die pflegerische Betreuung des Patienten pro Tag ist je nach Einstufung in eine

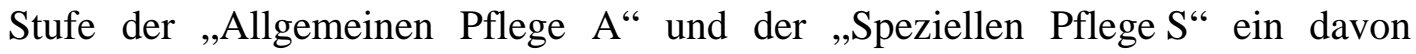
abhängiger, spezifischer pflegerischer Zeitaufwand gegeben. ${ }^{376}$

Für die 18 Tage Verweildauer auf der Normalstation ergibt sich bei einer Gewichtung der Pflegetage nach der Pflegepersonalregelung in PPR-Minuten folgende fallbezogene zeitliche Bindung des Pflegepersonals einschließlich des

Fallwertes:

\begin{tabular}{|l|l|l|}
\hline A1/S1 & $3 \mathrm{PT} \times 82 \mathrm{~min} / \mathrm{PT}$ & $246 \mathrm{Min}$. \\
\hline $\mathrm{A} 2 / \mathrm{S} 1$ & $1 \mathrm{PT} \times 128 \mathrm{~min} / \mathrm{PT}$ & $128 \mathrm{Min}$. \\
\hline $\mathrm{A} 3 / \mathrm{S} 1$ & $9 \mathrm{PT} \times 209 \mathrm{~min} / \mathrm{PT}$ & $1.881 \mathrm{Min}$. \\
\hline $\mathrm{A} 3 / \mathrm{S} 2$ & $4 \mathrm{PT} \times 219 \mathrm{~mm} / \mathrm{PT}$ & $876 \mathrm{Min}$. \\
\hline $\mathrm{A} 3 / \mathrm{S} 3$ & $1 \mathrm{PT} \times 245 \mathrm{~min} / \mathrm{PT}$ & $245 \mathrm{Min}$. \\
\hline Einmaliger Fallwert je Fallpauschale & $70 \mathrm{Min}$. \\
\hline Gesamtminuten pflegerische Betreuung & $3.446 \mathrm{Min}$. \\
\hline
\end{tabular}

- Ermittlung des Kostensatzes:

$\begin{array}{cc}\text { Personalkosten PD u.a. } & \text { 13.604.660 DM } \\ \text { Pflegeminuten p.a. } & \text { 16.208.063 Min. }\end{array}=0,84$ Dm/min.

Ermittlung der Fallkosten:

\subsection{Min $\times 0,84 \mathrm{DM} / \mathrm{Min} .=2.894 .64 \mathrm{DM}$}

Gesamte Personalkosten der Normalstation $(\mathrm{i}+\mathrm{ii})$ :

$$
455,06 \mathrm{DM}+2.894,64 \mathrm{DM}=\underline{3.349,68 \mathrm{DM}}
$$

\section{iii. Sachkosten:}

Mengenkomponente 18 Pflegetage:

Mit der Schlüsselung über die Verweiltage werden die Sachmittel ermittelt: ${ }^{377}$

Ermittlung des Kostensatzes: 23,64 DM/PT

376 Für die neun möglichen Merkmalskombinationen der Einstufungen sind die zeitlichen Bindungen des Pflegepersonals zusammengesetzt aus einen spezifischen pflegerischen Zeitaufwand (Spezielle Pflege ,S“) pro Tag zusammengestellt, der um einen einheitlichen Grundwert für allgemeine Pflege (Allgemeine Pflege ,A“) pro Tag in Höhe von 30 Minuten ergänzt wird. Die folgende Tabelle stellt die zeitliche Bindung des Pflegepersonals nach PPR-Regelung. Vgl. Baugut, G. (1995), S. 154.

\begin{tabular}{|l|l|l|l|}
\hline & S1 & S2 & S3 \\
\hline A1 & $52+30=82 \mathrm{~min}$ & $62+30=92 \mathrm{~min}$ & $88+30=118 \mathrm{~min}$ \\
\hline A2 & $98+30=128 \mathrm{~min}$ & $108+30=164 \mathrm{~min}$ & $134+30=164 \mathrm{~min}$ \\
\hline A3 & $179+30=209 \mathrm{~min}$ & $189+30=219 \mathrm{~min}$ & $215+30=245 \mathrm{~min}$ \\
\hline
\end{tabular}

377 Der pro Patient eingesetzte Sachbedarf kann entweder durch die unmittelbare Erfassung mittels Verbrauchsstücklisten oder durch die Schlüsselung über eine geeignete Bezugsgröße bestimmt werden, wobei ersteres nur für hochwertige sogenannte A-Artikel letzteres für geringwertige gilt B- und C-Artikel auf den Stationen, in den Funktionsbereichen und im Bereich Basisleistungen. Für die Kostenarten Instandhaltung, Medizintechnik und Gebrauchsgüter Medizintechnik kann die Schlüsselung (im Stationsbereich nach Verweildauer, im OP-Bereich nach OP-Zeiten und im Funktionsbereich anhand der Leistungspunkte nach dem GOÄ Leistungsverzeichnis) erfolgen. 
Ermittlung der Fallkosten: $18 \mathrm{PT} \times 23,64 \mathrm{DM} / \mathrm{PT}=\underline{425,52 \mathrm{DM}}$

Gesamte Kosten der Normalstation je Fall:

Personalkosten
Sachkosten

Gesamtkosten
3.349,68 DM

$425,52 \mathrm{DM}$

$\underline{\underline{3.775,20 \mathrm{DM}}}$

b. Intensivstation:

i. Arztdienst und Funktionsdienst:

Mengenkomponente: 24 St. Behandlung auf der Intensivstation):

Zur Darstellung des Zeitaufwandes des ärztlichen Dienstes und des Funktionsdienstes eines Falls wird angenommen, dass die Verweildauer des Patienten auf der Intensivstation in Belegungszeit nach Betreuungskategorien in Stunden notiert wird. $^{378}$

- Ermittlung des Kostensatzes:

Belegstunden gesamt/ Jahr 39.168 Std.

(davon 30\% Überwachung, 40\% Behandlung und 30\% Beatmung)

Kosten Arztdienst p.a.: $\quad 285.785$ DM

Kosten Pflegedienst p.a.: 1.026 .781 DM

Kostensatz Arztdienst (IntensivArztstunden p.a.):

$(0,3 \times 0,67+0,4 \times 1,0+0,3 \times 1,0) \times 39 \cdot 168$ Std. p.a. $=35 \cdot 290$

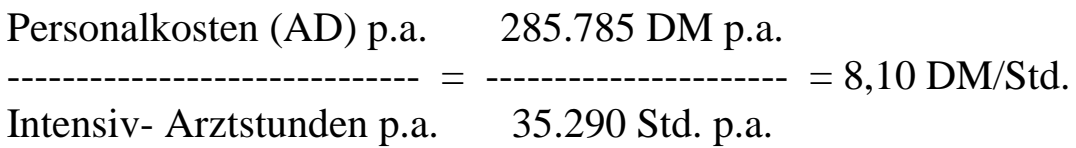

Kostensatz Pflegedienst (Intensiv-Pflegestunden p.a.):

$(0,3 \times 0,57+0,4 \times 1,0+0.3 \times 1,71) \times 39.168$ Std. p.a. $=42.458$

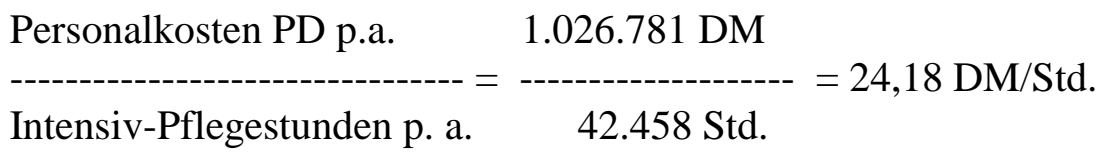

- Ermittlung der Fallkosten:

378 Es wird zwischen Überwachung, Behandlung und Beatmung unterschieden. Die Verweildauerstunden unterschiedlicher Versorgungskategorien werden anhand einer Umrechnung der Überwachungs- und Beatmungsstunden zusammengestellt, die Bewertung von Zeitaufwendungen auf der Intensivstation mittels Äquivalenzziffern dargestellt. Vgl. Baugut, G. (1995), S. 155.

\begin{tabular}{|l|l|l|l|}
\hline & Überwachung & Behandlung & Beatmung \\
\hline Arztdienst & 0,67 & 1,0 & 1,0 \\
\hline Pflegedienst & 0,57 & 1,0 & 1,71 \\
\hline
\end{tabular}


Personalkosten:

$$
\begin{aligned}
& \text { Arztdienst: } 24 \text { Stunden } \times 8,10 \text { DM/Std. }=194,40 \mathrm{DM} \\
& \text { Pflegedienst: } 24 \text { Stunden } \times 24,18 \mathrm{DM} / \text { Std. }=580,32 \mathrm{DM} \\
& \underline{774,72 \mathrm{DM}}
\end{aligned}
$$

ii. Sachkosten:

$$
24 \text { Stunden } \times 12,96 \mathrm{DM} / \mathrm{Std} .=\underline{311,04 \mathrm{DM}}
$$

Gesamt Direktkosten der Intensivstation je Fall (i + ii):

$$
\begin{array}{lr}
\text { Personalkosten } & 774,72 \mathrm{DM} \\
\text { Sachkosten } & 311,04 \mathrm{DM} \\
& -------- \\
\text { Gesamtkosten } & 1.085,76 \mathrm{DM}
\end{array}
$$

B. Operationsleistungen:

Sie umfassen den Personaleinsatz des ärztlichen und pflegerischen Operations- und Anästhesiedienstes im OP-Bereich und gegebenenfalls Leistungen des medizinischtechnischen Dienstes.

\section{a. Operationsdienst:}

Mengenkomponente: Die Mengenkomponente des Personaleinsatzes im OP bestimmt sich aus der Operationszeit, der Rüstzeit für den Funktions- und Arztdienst sowie der Anzahl der beteiligten Arzte und Schwestern (Gleichzeitigkeitsfaktor).

Netto-OP-Zeit(Schnitt-/Nahtzeit) : $\quad 75$ Min.

Rüstzeit (Zeit der Vorbereitung des Patienten ${ }^{379}$ ): 30 Min.

Gleichzeitigkeitsfaktor operativer Arztdienst: $\quad 3,0$

Gleichzeitigkeitsfaktor operativer Funktionsdienst: 2,5

Personaleinsatz (Arztdienst):

315 Min.

Personaleinsatz (Funktionsdienst): $\quad 263$ Min.

- Ermittlung des Kostensatzes:

Personalkosten Ärztlicher Dienst p.a.: $\quad$ 888.488 DM

Personalkosten Funktionsdienst p.a.: $\quad 781.910$ DM

OP-Zeit p.a. bei 2,5 Ärzten FKD p.a.: $\quad 282.600$ Min.

Kostensatz Ärztlicher Dienst:

${ }^{379}$ Die Vorbereitung eines OP-Patienten kann Maßnahmen wie Waschen, Desinfizieren und Lagern des Patienten umfassen. 


$$
\begin{aligned}
& \text { Personalkosten (AD) p.a. } \quad \text { 888.488 DM } \\
& \text { - } \\
& \text { Personaleinsatz p.a. } \quad 2,5 \times 282.600 \text { Min. }
\end{aligned}
$$

Kostensatz Funktionsdienst:

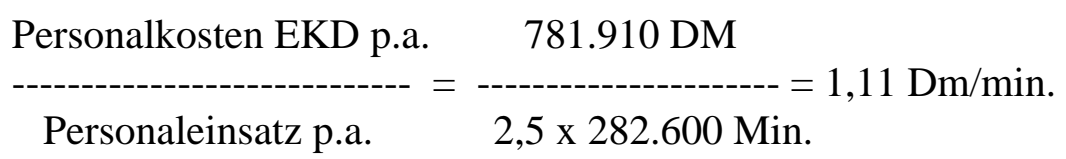

- Ermittlung der Fallkosten:

$$
\begin{array}{lr}
\text { Arztdienst: } & 315 \text { Min. } \times 1,26 \text { DM/Min. }=396,90 \mathrm{DM} \\
\text { Funktionsdienst: } & 263 \text { Min. } \times 1,11 \mathrm{DM} / \text { Min. }=291,93
\end{array}
$$

$\mathrm{DM}$

$\underline{688,83 \mathrm{DM}}$

b. Anästhesiologischer Arzt- und Funktionsdienst:

Für den Anästhesie-Bereich gilt dementsprechend die Anästhesiezeit wie im OP-Bereich als Maßstab der Kostenverteilung.

Mengenkomponente (Anästhesiezeit: 119 Min):

- Ermittlung der Kostensatz:

Anästhesiezeit p.a.: 533.880 Min.

Personalkosten Arztdienst p.a.: 1.436.352 DM

Personalkosten Funktionsdienst p. a.: $\quad$ 703.638 DM

Kostensatz Arztdienst: 2,69 Dm/min.

Kosten Funktionsdienst: $1,32 \mathrm{DM} / \mathrm{Min}$.

- Ermittlung der Fallkosten:

Arztdienst: $\quad 119 \mathrm{Min} . \times 2,69 \mathrm{DM} / \mathrm{Min} .=320,11 \mathrm{DM}$

Funktionsdienst: 119 Min. $\times 1,32$ DM/Min. $=157,08 \mathrm{DM}$

$477.09 \mathrm{DM}$

c. OP-Anästhesie-Sachkosten:

Gesamte Fallkosten: (OP und Anästhesie):

$\underline{2.798,16 \mathrm{DM}}$

(Davon: 1.270 DM Implantate)

Gesamte Direktkosten der OP und Anästhesie $(a+b+c): \underline{3.874,18 \text { DM }}$ 


\section{Funktionsleistungen der Medizinischen Institutionen:}

Sie umfassen die aus dem Stationsbereich oder auch aus dem OP-Bereich von medizinischen Institutionen bezogenen Funktionsleistungen, d. h. Leistungen des Labors oder der Röntgendiagnostik und anderer Kostenstellen des Untersuchungsund Behandlungsbereiches Medizinischer Institutionen des eigenen Krankenhauses. Mengenkomponente: 9.188 Punkte.

Es ist patientenbezogen $\mathrm{zu}$ dokumentieren, welche Leistungen aus dem Bereich Normal- und Intensivstation bzw. aus dem Operationsbereich von medizinischen Institutionen des Krankenhauses angefordert werden. ${ }^{380}$

Ermittlung des Kostensatzes:

hausindividuell ermittelter Satz von 0,07 DM/Punkt

Ermittlung der Fallkosten:

$$
\text { 9.188 Punkte } \times 0,07 \mathrm{DM} / \text { Punkt }=\underline{\underline{643.16 \mathrm{DM}}}
$$

\section{Basis-Leistungen:}

Sie umfassen die Leistungen und Kostenstellen des nicht-medizinischen Infrastrukturbereiches wie die Patientenaufnahme sowie weitere Wirtschafts-, Versorgungs- und Verwaltungsdienste.

Mengenkomponente: 19 Pflegetage ${ }^{381}$

Ermittlung des Kostensatzes:

Personalkostensatz: $79,56 \mathrm{DM} / \mathrm{PT}$

Sachkostensatz: 63,56 DM/PT

Ermittlung der Fallkosten:

\begin{tabular}{rlr}
$19 \mathrm{PT} \times 79,56 \mathrm{DM} / \mathrm{PT}$ & $=$ & $1.511,64 \mathrm{DM}$ \\
$19 \mathrm{PT} \times 63,56 \mathrm{DM} / \mathrm{PT}$ & $=$ & $1.207 .64 \mathrm{DM}$ \\
& & $\underline{\underline{2.719,28 \mathrm{DM}}}$ \\
\hline
\end{tabular}

380 Es kann sich um Untersuchungs- und Behandlungsleistungen im Bereich Labor, Radiologie, EKG, Sonographie sowie physikalische Therapie handeln. Am Beispiel der Operation einer Schenkelhalsfraktur wurden von den medizinischen Institutionen Leistungen erbracht, die bei einer Bewertung nach den Leistungsverzeichnissen der GOÄ in der Summe 9.188 Punkte für diesen Patienten ergeben.

381 Die Leistungen der Bereiche Wirtschaft, Versorgung und Verwaltung werden aus Vereinfachungsgründen anhand der Verweildauer geschlüsselt. Es ist bei einer hinreichend differenzierten Datenerhebung auch möglich, die Kosten fallbezogen zu schlüsseln. Wegen des inhaltlichen Zusammenhangs dieses Kostensatzes für die Entgeltermittlung des Basispflegesatzes wird auf diese Differenzierungsmöglichkeit jedoch verzichtet. 


\begin{tabular}{|l|l|l|l||}
\hline $\begin{array}{l}\text { Kostenstellenbereic } \\
\text { he }\end{array}$ & Personalkosten & Sachkosten & Gesamte Fallkosten \\
\hline $\begin{array}{l}\text { A. Stationsleistungen } \\
\text { - Normalstation } \\
\text { - Intensivstation }\end{array}$ & $\begin{array}{l}3.349,68 \mathrm{DM} \\
774,72 \mathrm{DM}\end{array}$ & $\begin{array}{l}425,52 \mathrm{DM} \\
311,04 \mathrm{DM}\end{array}$ & $\begin{array}{l}3.775,20 \mathrm{DM} \\
1.085,76 \mathrm{DM}\end{array}$ \\
\hline $\begin{array}{l}\text { B. OP- Leistungen: } \\
\text { - OP-Bereich } \\
\text { - Anästhesie }\end{array}$ & $\begin{array}{l}1.166 .02 \mathrm{DM} \\
688,83 \mathrm{DM} \\
477,09 \mathrm{DM}\end{array}$ & $2.708,16 \mathrm{DM}$ & $3.874,18 \mathrm{DM}$ \\
\hline $\begin{array}{l}\text { C. } \\
\begin{array}{l}\text { Funktionsleistung } \\
\text { en in med. } \\
\text { Institution. }\end{array}\end{array}$ & $471,72 \mathrm{DM}$ & $171,44 \mathrm{DM}$ & $643,16 \mathrm{DM}$ \\
\hline \begin{tabular}{l} 
D. Basis-Leistungen \\
\hline \begin{tabular}{l} 
Gesamt \\
\hline
\end{tabular}
\end{tabular} & $7.511,64 \mathrm{DM}$ & $1.207,64 \mathrm{DM}$ & $2.719,28 \mathrm{DM}$ \\
\hline
\end{tabular}

Tab 13: Kalkulationsergebnisse für die Abbildung 27: Zusammenstellung der Fallpauschale Nr. 17.02.

\subsection{Kalkulation von Sonderentgelten:}

Die Kalkulation von Sonderentgelten beinhaltet die Ermittlung der Pflegesatzrelevanten Kosten für Leistungen eines festgelegten Behandlungsfalls. ${ }^{382}$ Die Behandlungsfälle betreffen meistens den operativen Bereich und sind mit entsprechenden Bewertungsrelationen in der Anlage 2 der BPflV katalogisiert. ${ }^{383}$

Für die Kalkulation von Sonderentgelten nach der Vorgabe der BPflV sind grundsätzlich lediglich die Kosten des ärztlichen Dienstes, des Pflegedienstes, des medizinisch-technischen Dienstes, des Funktionsdienstes und medizinischen Bedarfs zu berücksichtigen. ${ }^{384}$ Das Kalkulationsschema für die Bestimmung von Sonderentgelten für operative Behandlungsfälle ist in Tab. 14 dargestellt.

\footnotetext{
${ }^{382}$ Vgl. § 11 Abs. 2 BPflV.

383 Der Katalog sieht rund 100 Sonderentgelte vor. Diese Sonderentgelte betreffen fast ausschließlich operative Leistungen. Nur 4 davon beziehen sich auf konservative Diagnostik bzw. Interventionen. Vgl. Anlage 2 BPflV.

384 Vgl. Deutsche Krankenhausgesellschaft (1993): Sonderentgelte und Fallpauschalen gefährden die bürgernahe Krankenhausversorgung- Stellungnahme der DKG zur „Grundkonzeption des
} 


\begin{tabular}{|c|c|c|}
\hline $\begin{array}{l}\text { Kalkulations } \\
\text { schritte }\end{array}$ & $\begin{array}{l}\text { Zeil } \\
\text { e }\end{array}$ & Kalkulationselemente \\
\hline $\begin{array}{l}\text { Ermittlung des } \\
\text { Mengengerüst } \\
\text { es }\end{array}$ & $\begin{array}{l}1 \\
2 \\
3 \\
4\end{array}$ & $\begin{array}{l}\text { Mittlere Schnitt-/Nahtzeit (mittlere Operationsdauer) } \\
\text { Mittlere Ein-/Ausleitzeit (mittlere Anästhesiedauer) } \\
\text { Mittlere Rüstzeit der Ärzte des operativen Dienstes } \\
\text { Mittlere Vor- und Nachbereitungszeit des op. } \\
\text { Funktionsdienstes }\end{array}$ \\
\hline $\begin{array}{l}\text { Ermittlung } \\
\text { des } \\
\text { Wertgerüstes } \\
\text { (Kostensätze) }\end{array}$ & $\begin{array}{l}5 \\
6 \\
7 \\
8\end{array}$ & $\begin{array}{l}\text { Kosten je Operationsminute (Ärzte des operativen } \\
\text { Dienstes) } \\
\text { Kosten je Operationsminute (operativer Funktionsdienst) } \\
\text { Kosten je Anästhesieminute (Ärzte der Anästhesie) } \\
\text { Kosten je Anästhesieminute (Funktionsdienst der } \\
\begin{array}{l}385 \\
\text { Anästhesie) }\end{array}\end{array}$ \\
\hline $\begin{array}{l}\text { Ermittlung } \\
\text { der Personal- } \\
\text { kosten0 }\end{array}$ & $\begin{array}{l}9 \\
10 \\
11 \\
12\end{array}$ & $\begin{array}{l}\text { Kosten der Ärzte des operativen Dienstes [(Zeile } 1+\text { Zeile } \\
3) \times \text { Zeile 5] } \\
\text { Kosten des operativen Funktionsdienstes [(Zeile } 1+\text { Zeile } 4) \\
\times \text { Zeile } 6 \text { ] } \\
\text { Kosten der Ärzte des Anästhesiedienstes [(Zeile } 2+\text { Zeile 7] } \\
\text { Kosten des Funktionsdienstes der Anästhesie (Zeile 2 + } \\
\text { Zeile } 8)\end{array}$ \\
\hline Zwischensumm & 13 & $\begin{array}{l}\text { Summe der Personalkosten(Zeile } 9+\text { Zeile } 10+\text { Zeile } 11+ \\
\text { Zeile 12) }\end{array}$ \\
\hline $\begin{array}{l}\text { Ermittlung } \\
\text { der Kosten für } \\
\text { Sachbedarf }\end{array}$ & $\begin{array}{l}14 \\
15 \\
16 \\
17 \\
18\end{array}$ & $\begin{array}{l}\text { Implantate/ Transplantate } \\
\text { Blut/ Blutersatzmittel } \\
\text { Medikamente } \\
\text { Sonstiger medizinischer Bedarf } \\
\text { Wäsche und Abdeckungen }\end{array}$ \\
\hline $\begin{array}{l}\text { Zwischensumn } \\
\mathrm{e}\end{array}$ & 19 & $\begin{array}{l}\text { Summe der Sachkosten (Zeile } 14+\text { Zeile } 15+\text { Zeile } 16+ \\
\text { Zeile } 17+\text { Zeile } 18 \text { ) }\end{array}$ \\
\hline $\begin{array}{l}\text { Kalkulations- } \\
\text { ergebnis }\end{array}$ & 20 & Summe der Personal- und Sachkosten (Zeile $13+$ Zeile 19) \\
\hline
\end{tabular}

Tab. 14: Das Kalkulationsschema für die Ermittlung eines Sonderentgeltes (OP-Bereich). ${ }^{387}$

neuen Entgeltsystems“ des Bundesministeriums für Gesundheit, in das Krankenhaus, 4 /1993, S. 157.

385 Da die Operateure nur einen Teil ihrer Dienstzeit im OP- Bereich verbringen, sollte die Ermittlung des Personalkostensatzes auf der Basis von Anwesenheitszeiten im OP- ereich erfolgen.

${ }^{386}$ Da normalerweise des Personals des operativen Funktionsdiensts, die Ärzte des Anästhesiediensts und die Pflegepersonal des Funktionsdienstes der Anästhesie nur im OP-Bereich tätig sind, sollen für die Ermittlung des jeweiligen Personalkostensatzes die Leistungszeiten als Grundlage gewählt werden.

${ }^{387}$ Quelle: Bundesministerium für Gesundheit (1993), S. 44. 


\subsection{Kalkulation von Abteilungspflegesatz und Basispflegesatz}

Bei der Ermittlung von Abteilungspflegesätzen und Basispflegesatz handelt es sich um Krankenhausleistungen, die nicht im Rahmen der Fallpauschalen- oder Sonderentgeltkalkulation berücksichtigt werden.

Innerhalb der Pflegesatzverhandlungen wird für jede organisatorisch eigenständige bettenführende Abteilung ${ }^{388}$ sowie besondere Einrichtungen des Krankenhauses ein spezifischer Abteilungspflegesatz vereinbart. ${ }^{389}$ Die Abteilungspflegesätze stellen das wertmäßige Äquivalent für ärztliche und pflegerische Leistungen auf den Stationen und zugeordnete Kosten von innerbetrieblichen Leistungen dar. Werden z. B. Labor- und Röntgenleistungen auf einen Pflegetag bezogen, ist der Basispflegesatz das Ergebnis der kostenmäßigen Erfassung von nicht-medizinischen oder pflegerischen Leistungen der Bereiche Unterkunft, Verpflegung, Verwaltung und Instandhaltung bezogen auf einen Pflegetag. ${ }^{390}$

Die Abteilungspflegesätze sind die Grundlage zur Ermittlung der Kostenträgerstückkosten für die künstlichen Kostenträger (Berechnungstage). Zur Ermittlung von einem Abteilungspflegesatz müssen die nicht pflegesatzfähigen Kosten von den gesamten Kosten der Abteilung ${ }^{391}$ gemäß dem Nettoprinzip der LKA ausgegliedert werden. Von den sich daraus ergebenen Kosten (Pflegesatzfähigen Kosten) müssen die anteilig in den Kostenstellen erbrachten Fallpauschalen und Sonderentgelte abgezogen werden. Danach wird der Pflegesatz der Abteilung von den verbleibenden Pflegesatzfähigen Kosten und dem Anzahl der Berechnungstage mit dem Verfahren der Divisionskalkulation ermittelt. Tab. 15 stellt das Kalkulationsschema für die Ermittlung eines Abteilungspflegesatzes dar.

${ }^{388}$ Nach $§ 13$ Abs. 2 sind diese Einrichtungen diejenige, die ausschließlich oder überwiegend der Behandlung von Querschnittsgelähmten, Schwerst-Schädel-Hirn-Verletzten, Schwerbrandverletzten, AIDS-Patienten, Transplantationspatienten, Dialysepatienten oder der neonatologischen Intensivbehandlung von Säuglingen dienen.

389 Für Fachbereiche mit sehr geringer Bettenzahl kann ein gemeinsamer Belegpflegesatz vereinbart werden. Vgl. § 13, Abs. 2 und Abs. 3 BPflV.

${ }^{390}$ Vgl. Preuß, O. (1996), S. 74. Und Rossels, H., (1994): Das neue Pflegesatzrecht, lohnt sich der Einsteig schon ab 1995, Contra-Argumentation, in: F\&W, 11 Gangjahr, 1994, S. 289.

391 Die gesamten Kosten einer Abteilung setzen sich sowohl aus den eigentlichen Primärkosten als auch aus Sekundärkosten der innerbetrieblichen Leistungsverrechnung zusammen. 


\begin{tabular}{|c|c|c|}
\hline Kalkulationsschritte & Zeile & Kalkulationselemente \\
\hline $\begin{array}{l}\text { Ermittlung der primären } \\
\text { Kosten: } \\
-\quad \text { Personal kosten } \\
-\quad \text { Sachkosten } \\
\end{array}$ & 1 & $\begin{array}{l}\text { Ärztlicher Dienst, Pflegedienst, technischer } \\
\text { Dienst med. Bedarf, Instandhaltung u. } \\
\text { Gebrauchsgüter }\end{array}$ \\
\hline $\begin{array}{l}\text { Ermittlung der } \\
\text { sekundären Kosten }\end{array}$ & 2 & $\begin{array}{l}\text { Intensiv-, OP-Bereich, Medizinische } \\
\text { Institutionen }\end{array}$ \\
\hline Gesamten Kosten & 3 & $(1+2)$ \\
\hline $\begin{array}{l}\text { Abzüge: Ausgliederung } \\
\text { der } \\
\text { Pflegesatzfähigen } \\
\text { Kosten }\end{array}$ & 4 & $\begin{array}{l}\text { Abzüge nach } § 7 \text { Abs. } 2 \text { BPflV: } \\
-\quad \text { Vor- und Nachstationären Behandlung } \\
-\quad \text { Wahlärztliche Leistungen }\end{array}$ \\
\hline $\begin{array}{l}\text { Pflegesatzfähigen } \\
\text { Kosten }\end{array}$ & 5 & $(4-3)$ \\
\hline $\begin{array}{l}\text { Abzüge: Ausgliederung } \\
\text { der Kostenstellen }\end{array}$ & 6 & $\begin{array}{l}\text { Der Kostenstelle zuzurechnende Anteil der } \\
\text { Fallpauschalen und Sonderentgelte }\end{array}$ \\
\hline $\begin{array}{l}\text { Verbleibenden } \\
\text { Pflegesatzfähigen } \\
\text { Kosten }^{392}\end{array}$ & 7 & $(6-5)$ \\
\hline Berechnungstage & 8 & Anzahl der Berechnungstage der Abteilung. \\
\hline $\begin{array}{l}\text { Vollstationärer } \\
\text { Abteilungspflegesatz }\end{array}$ & 9 & (7/ 8) (DM /Tag) \\
\hline
\end{tabular}

Tab. 15: Das Kalkulationsschema für Ermittlung eines Abteilungspflegesatzes. $^{393}$

\subsubsection{Durchführung der Kostenträgerzeitrechnung}

Bei der Durchführung der Kostenträgerzeitrechnung soll das Krankenhausergebnis durch die Gegenüberstellung von Leistungen und Kosten für eine Periode aufgezeigt werden (kurzfristige Erlösrechnung). Analog zur Kostenrechnung beschäftigt sich die Erlösrechnung im deutschen Krankenhaus mit der Erfassung von einzelnen Erlöspositionen und deren Verrechnung auf die Erlösträger. Die Kalkulation des Krankenhaus-Budgets mittels Kosten- und Leistungsnachweises (KLN) kann als Kostenträgerzeitrechnung für das deutsche Krankenhaus als Ganzes verstanden

\footnotetext{
392 Die verbleibenden pflegesatzfähigen Kosten, die die Budgetanteil des Vollstationären Abteilungspflegesatzes sind, werden über das krankenhausindividuelle Restbudget vergütet. Vgl. 2.2.3.2.

393 In Anlehnung an: Vgl. Maltry, H., Strehlau-Schwoll, H. (1997), S. 550.
} 
werden. Abb. 27 stellt das Rechenschema für die Durchführung der Kostenträgerzeitrechnung für eine einzelne bettenführende Abteilung dar. Die Zusammenfassung der Teilergebnisse der Abteilungen führt zum Gesamtbetriebsergebnis.

\begin{tabular}{||l||}
\hline Betriebliche Gesamtleistung \\
./. Personalaufwand \\
= Teilergebnis I \\
./. Lebensmittel, medizinischer Bedarf und sonstige Sachkosten \\
\hline = Teilergebnis II \\
+ Innerbetriebliche Leistungsverrechnung \\
= Teilergebnis III \\
+ Zuweisungen und andere Erträge \\
= Ordentliches Betriebsergebnis IV \\
\hline
\end{tabular}

Abb. 27: Das Kalkulationsschema der Kostenträgerzeitrechnung 394

Die Erlösarten für Zwecke der kurzfristigen Erfolgsrechnung bei dem geförderten Krankenhaus in Deutschland werden entsprechend den Bestimmungen der KHBV in der Finanzbuchhaltung, Kontenklasse 4, erfasst ${ }^{395}$ (Abb. 28). Sie ergeben sich aus den rechtlichen Entgeltsformen der Krankenhausleistungen, es sind sowohl Erlöse aus allgemeinen budgetorientierten Leistungen in Form von Fallpauschalen, Sonderentgelten, Basispflegesatz und Abteilungspflegesätzen $\S 11, \S 12$ und $\S 13$ BPflV als auch Erlöse zur Deckung der ausgegliederten Kosten nach $§ 7$ BPflV, z. B. Erlöse aus Wahlleistungen und Erlöse aus vor- und nachstationären Behandlungen.

394 Quelle: Hildbrand, R. (1988), S. 416.

395 Erlöse bei nicht geförderten Krankenhäusern können dagegen in Erlöse von Krankenkassen, Erlöse von Privatpatienten und Erlöse von Nicht-Patienten (Gäste) gegliedert werden. Vgl. Koch, J. (1998): Gesundheitsökonomie, Betriebswirtschaftliche Kosten- und Leistungsrechnung, München, Wien, Oldenburg, 1998, S. 121. 


\begin{tabular}{|c|c|}
\hline $\begin{array}{l}\text { Konten } \\
\text { Nr. }\end{array}$ & Erlöse Arten \\
\hline $\begin{array}{l}40 \\
400 \\
4001 \\
4002 \\
4003 \\
4004 \\
4005 \\
4006 \\
401 \\
4010 \\
4011 \\
402 \\
4020 \\
4021 \\
41 \\
410 \\
411 \\
413 \\
42 \\
420 \\
4200 \\
4201 \\
4203 \\
42031 \\
42032 \\
421 \\
422 \\
43\end{array}$ & $\begin{array}{l}\text { Erlöse aus Krankenhausleistungen } \\
\text { Erlöse aus tagesgleichen Pflegpflegesätzen } \\
\text { Erlöse aus Basispflegesatz, vollstationär } \\
\text { Erlöse aus Basispflegesatz, teilstationär } \\
\text { Erlöse aus Abteilungspflegesätzen, vollstationär } \\
\text { Erlöse aus Abteilungspflegesätzen, teilstationäre } \\
\text { Erlöse aus Pflegesätzen für besondere Einrichtungen, } \\
\text { vollstationär } \\
\text { Erlöse aus Pflegesätzen für besondere Einrichtungen, } \\
\text { teilstationäre } \\
\text { Erlöse aus Fallpauschalen und Sonderentgelten } \\
\text { Erlöse aus Fallpauschalen } \\
\text { Erlöse aus Sonderentgelten } \\
\text { Erlöse aus vor- und nachstationärer Behandlung } \\
\text { Erlöse aus vorstationärer Behandlung nach } \S 115 \mathrm{a} \text { SGB V } \\
\text { Erlöse aus nachstationärer Behandlung } § 115 a \text { SGB V } \\
\text { Erlöse aus Wahlleistungen } \\
\text { Erlöse aus wahlärztlichen Leistungen } \\
\text { Erlöse aus gesondert berechenbarer Unterkunft nach } \S 22 \\
\text { BPflV } \\
\text { Erlöse aus sonstigen nichtärztlichen Wahlleistungen } \\
\text { Erlöse aus ambulanten Leistungen des Krankenhauses } \\
\text { Erlöse aus Krankenhausambulanzen } \\
\text { Notfallbehandlung } \\
\text { Institutsermächtigungen } \\
\text { Erlöse aus ambulanten Leistungen der physikalischen Therapie } \\
\text { Von Sozialleistungsträgern } \\
\text { Von Selbstzahlern } \\
\text { Erlöse aus vor- und nachstationärer Behandlung } \\
\text { Erlöse aus ambulantem Operieren nach } § 115 b \text { SGB V } \\
\text { Nutzungsentgelte (Kostenerstattung und Vorteilsausgleich) } \\
\text { und sonstige Abgaben der Ärzte } \\
\text { Nutzungsentgelte für wahlärztliche Leistungen } \\
\text { Nutzungsentgelte für von Ärzten berechnete ambulante ärztliche } \\
\text { Leistungen }\end{array}$ \\
\hline
\end{tabular}

Abb. 28: Erlösarten im Krankenhaus ${ }^{396}$

396 Quelle: Anlage 4 zur KHBV. 


\subsubsection{Leistungsrechnung in den deutschen Krankenhäusern}

\subsubsection{Definition und Aufgaben der Leistungsrechnung in deutschen Kran- kenhäusern}

Die Leistungsrechnung des deutschen Krankenhauses spiegelt die erbrachten Leistungen in den einzelner Kostenstellen bzw. Leistungsbereichen differenziert nach stationärer und ambulanter Behandlung wieder. Sie dient sowohl internen als auch externen Aufgaben.

Intern hat die Leistungsrechnung die Aufgabe, einerseits die Ermittlung der Grundlagen der individuellen Leistungsfähigkeit des Krankenhauses zur Beurteilung der Wirtschaftlichkeit sowie zur Durchführung innerbetrieblicher Kostenkontrolle, andererseits die Ermittlung der sachgerechten Verteilungsschlüssel (Verteilung der Kosten der leistungserbringenden Kostenstellen auf die leistungsempfangenden Kostenstellen) durch die mengen- und wertmäßige Erfassung von innerbetrieblichen Leistungen durchzuführen.

Extern dienen die im Rahmen der Leistungsrechnung erfaßten Leistungsmengen als Grundlage für die Abrechnung dieser Leistungen mit den Kostenträgern (Patienten, gesetzliche Krankenkassen, private Krankenversicherungen oder sonstige Dritte). Andererseits dient die Leistungsrechnung zur Erfüllung der rechtlichen Erfordernisse, wobei jedes deutsche Krankenhaus nach $\S 8$ KHBV zur Führung einer Leistungsrechnung verpflichtet ist, die eine Beurteilung der Wirtschaftlichkeit und Leistungsfähigkeit ermöglichen. ${ }^{397}$

\subsubsection{2: Erfassung und Bewertung der Leistungen in deutschen Kranken- häusern}

Die Leistungsdokumentation in den deutschen Krankenhäusern hat sich auf die vom Gesetzgeber vorgeschriebene Mindestanforderungen beschränkt. ${ }^{398}$ Gemäß dem gesetzlich geforderten Kostenzurechnungskonzept nach $\S 8$ KHBV sieht dieses in einigen Krankenhausbereichen eine äußerst genaue Leistungserfassung

397 Erst eine Gegenüberstellung der Kosten und Leistungen ermöglicht Rückschlüsse auf die Angemessenheit von Personal- und Sachkostenentwicklungen. Vgl. Deutsche Krankenhausgesellschaft (1992). S. 177-178. 
vor, ${ }^{399}$ wonach die Leistungen wie die Kosten verursachungsgerecht nach Kostenstellen (Kontenrahmen Nr. 9) zu erfassen sind. ${ }^{400}$ Davon sind nur die in den Pflegefachbereichen erbrachten ärztlichen und pflegerischen Einzelleistungen (Kontengruppen Nr. 93 u. 95) ausgenommen worden, ${ }^{401}$ weil der Aufwand ihrer Erfassung zu aufwendig und wirtschaftlich nicht vertretbar ist. ${ }^{402}$ Für Zwecke der Leistungsrechnung sollen die Leistungen eines Krankenhauses nach bestimmten Maßeinheiten erfasst werden. Das stellt Tab. 16 dar.

\begin{tabular}{|c|c|}
\hline Kostenstellen & Art der zu erfassenden Leistungen \\
\hline Werkstätten & Zahl der Arbeitsstunden \\
\hline Speisenversorgung & $\begin{array}{l}\text { Zahl der Beköstigungstage, unterteilt nach } \\
\text { Patienten, Personal und Sonstige sowie nach } \\
\text { Normalkost, Schonkost und Diätkost }\end{array}$ \\
\hline Wäscheversorgung & Kilogramm Schmutzwäsche (trocken) \\
\hline Bettenzentrale & $\begin{array}{l}\text { Zahl der gereinigten und aufgerüsteten } \\
\text { desinfizierten Betten }\end{array}$ \\
\hline Zentraler Reinigungsdienst & $\begin{array}{l}\text { Quadratmeter Nutzfläche, unterteilt nach der } \\
\text { Intensität der Reinigung und dem } \\
\text { Reinigungsturnus }\end{array}$ \\
\hline Innerbetriebliche Transporte & Zahl der Arbeitsstunden \\
\hline Lager & Verbrauch \\
\hline Apotheke & Arzneimittelverbrauch \\
\hline Zentrale Sterilisation & $\begin{array}{l}\text { Zahl der sterilisierten Güter in der örtlich fest } \\
\text { gelegten Zusammenstellung }\end{array}$ \\
\hline $\begin{array}{l}\text { Röntgendiagnostik und - } \\
\text { therapie } \\
\text { Nukleardiagnostik und -therapie }\end{array}$ & $\begin{array}{l}\text { Zahl der angeforderten und erbrachten } \\
\text { Leistungen } \\
\text { Gebührenverzeichnis der Gebührenordnung }\end{array}$ \\
\hline
\end{tabular}

398 Vgl Strehlau-Schwoll, H. (1993), S. 216.

399 Vgl. Schmidt, K. J., Heuser, U.: Das neue Fallpauschalen, Kalkulationsschema, Einfach aber ungenau, in: das Krankenhaus, 10/ 1993, S. 475.

${ }^{400}$ Vgl. $\$ 8$ Abs. 2 Nr. 3 KHBV.

401 Bundesministerium für Arbeit und Sozialordnung (Hrsg.): Konzept der Kosten- und Leistungsrechnung, Nr. 2.7.

402 Als Beispiel können die erfassten Leistungen der Kostenstelle „913 Versorgung mit Energie, Wasser, Brennstoffen“ nur mit einer Zuordnung nach der Verbrauchsmessung (in $\mathrm{kWh}, \mathrm{m}^{3}$, kcal) weiterverrechnet werden. 


\begin{tabular}{|c|c|}
\hline $\begin{array}{l}\text { Laboratorium } \\
\text { Funktionsdiagnostik } \\
\text { Sonstige diagnostische } \\
\text { Einrichtungen Anästhesie } \\
\text { OP- Bereich }\end{array}$ & $\begin{array}{l}\text { für Ärzte (GOÄ) bzw. dem Krankenhaustarif } \\
\text { der Deutschen Krankenhausgesellschaft für } \\
\text { ambulante Leistungen und stationäre } \\
\text { Nebenleistungen (DKG- NT) }\end{array}$ \\
\hline Kreißzimmer & $\begin{array}{l}\text { Zahl der Geburten, unterteilt nach } \\
\text { Spontangeburten, Vakuum- Extraktionen, } \\
\text { Zangengeburten und Schnittentbindungen }\end{array}$ \\
\hline $\begin{array}{l}\text { Physikalische Therapie } \\
\text { Sonstige therapeutische } \\
\text { Einrichtungen }\end{array}$ & $\begin{array}{lrr}\text { Zahl der } & \text { angeforderten und erbrachten } \\
\text { Leistungen } & \text { entsprechend } & \text { dem } \\
\text { Leistungsverzeichnis des DKG- NT } & \end{array}$ \\
\hline $\begin{array}{l}\text { Pathologie } \\
\text { Ambulanzen }\end{array}$ & $\begin{array}{l}\text { Zahl der angeforderten und erbrachten } \\
\text { Leistungen entsprechend dem } \\
\text { Gebührenverzeichnis der GOÄ. unterteilt nach } \\
\text { Leistungen des Krankenhausarztes, Notfall- } \\
\text { Leistungen als Institutsleistungen und } \\
\text { Institutsleistungen für sonstige Dritte. }\end{array}$ \\
\hline
\end{tabular}

Tab. 16: Leistungserfassung des Krankenhauses. ${ }^{403}$

Die erfassten Leistungen sollen in der Leistungsrechnung bewertet werden. in deutschen Krankenhäusern sollen die Leistungen in Form von Punktzahl unter einem Verfahren wie folgt bewertet werden: ${ }^{404}$ Nach den Ansätzen der GOÄ bzw. dem Krankenhaustarif der Deutschen Krankenhaus-Gesellschaft für ambulante Leistungen und stationäre Nebenleistungen (DKG-NT) (z. B. für Leistungen der Kontengruppen 92), oder der Leistungsrelation sowie nach Äquivalenzziffern, i.V.m. § 16 BPflV, auf Grund von Arbeitsablauf- und Zeitanalysen oder abgeleitet aus dem Verhältnis der Ansätze einer Gebührenordnung bzw. eines Tarifes oder anderer Maßstäbe (z. B. Bundesmantelvertrag Ärzte (BMÄ) oder Einheitliche Bewertungsmaßstab für ärztliche Leistungen (EBM)) zueinander. ${ }^{405}$

\footnotetext{
403 In Anlehnung an: Bölke, G., Schmidt-Rettig, B., (1988), S. 463.

404 Die Punkte berücksichtigen die Gewichtung der einzelnen Leistungen, die Multiplikation der Punkte mit der Anzahl jeder Leistung ergibt die Gesamtpunktezahl einer Leistungsstelle.

405 § 16 Abs. 2 lautet: ,... Für die Entgelte sind Bewertungsrelationen in Form von Punktezahlen zu vereinbaren.“ $§ 16$ Abs. 4 lautet: „Die Vertragsparteien auf Landesebene geben den Vertragsparteien rechtzeitig die Punktwerte und die sich daraus ergebende Höhe der Entgelte, die nach Abs. 2 vereinbarten Entgelte und deren Bewertungsrelationen und Entgelthöhe sowie das Entgelt nach Abs. 3 nach deren Genehmigung bekannt. Vgl. Bölke, G., Schmidt- Rettig, B., (1988), S. 465-468.
} 


\subsubsection{Leistungsstatistik in den deutschen Krankenhäusern}

Daten und Informationen der im Krankenhaus erbrachten Leistungen, die gemäß $\S 17$ Abs. 4 BPflV und Anlage 3 BPflV in der Leistungs- und Kalkulationsaufstellung (LKA) gefordert sind, legen die Belegungsdaten des Krankenhauses (L1 und L3 der LKA), die Diagnosenstatistik (L4 der LKA) und die Operationsstatistik (L5 der LKA), Versorgungsstatistiken (S5 LKA) und Leistungsstatistiken für medizinischen Institutionen vor.

Die Teile L1 und L3 enthalten die in Abb. 29 dargestellten Belegungsdaten für das Krankenhaus insgesamt und differenziert nach Fachabteilungen. ${ }^{406}$

\begin{tabular}{|c|c|c|c|c|}
\hline \multirow[t]{2}{*}{$\begin{array}{l}\text { lfd. } \\
\text { Nr. }\end{array}$} & \multirow[t]{2}{*}{ Belegungsdaten } & \multirow{2}{*}{$\begin{array}{l}\text { Vereinbarun } \\
\text {; für deI } \\
\text { aufenden } \\
\text { ?flegesatz- } \\
\text { 'eitraum }\end{array}$} & \multicolumn{2}{|c|}{$\begin{array}{l}\text { Pflegesatzzeitrau } \\
\text { m }\end{array}$} \\
\hline & & & $\begin{array}{l}\text { Forde- } \\
\text { rung }\end{array}$ & $\begin{array}{l}\text { Verein- } \\
\text { barung }\end{array}$ \\
\hline & 1 & 2 & 3 & 4 \\
\hline $\begin{array}{l}1 \\
2 \\
3 \\
4 \\
5 \\
6 \\
7 \\
8 \\
9 \\
10 \\
11 \\
12 \\
13 \\
14 \\
15 \\
16 \\
17 \\
18\end{array}$ & $\begin{array}{l}\text { Planbetten mit Intensiv } \\
\text { Planbetten ohne Intensiv } \\
\text { Nutzungsgrad der Planbetten } \\
\text { BT im Budgetbereich } \\
\text { davon: BT für Patienten mit SE } \\
\text { davon: BT für teilstationäre } \\
\text { Patienten } \\
\text { Verweildauer (Nr.4: Nr.13 und 17) } \\
\text { Belegungstage FP-Bereich } \\
\text { Aufnahmen } \\
\text { Entlassungen } \\
\text { davon: Verlegungen nach außen } \\
\text { Fälle mit nur vorstat. Behandlung } \\
\text { Vollstat. Fälle im Budgetbereich } \\
\text { davon: Kurzlieger } \\
\text { davon: mit vorstationäre } \\
\text { Behandlung mit nachstationäre } \\
\text { davon: mit } \\
\text { Behandlung } \\
\text { Teilstat. Fälle m Budgetbereich } \\
\text { Fälle mit Fallpauschalen }\end{array}$ & & & \\
\hline
\end{tabular}

Abb. 29: Belegungsdaten des Krankenhauses ${ }^{407}$

\footnotetext{
406 Der Teil 2 enthält Statistiken über das Personal des Krankenhauses.

407 Quelle: Teil L1 Anlage 3 zu § 17 Abs. 4 BPflV.
} 
Teil L4 der LKA enthält die Diagnosenstatistik, insbesondere die Anzahl der Operationen, die Anzahl der je Fachabteilung entlassenen Patienten sowie Verweildauer und Alter und Struktur der Patienten.

Statistiken über die Versorgungsleistungen sind in Teil S5 der LKA (Abb. 30) dargestellt.

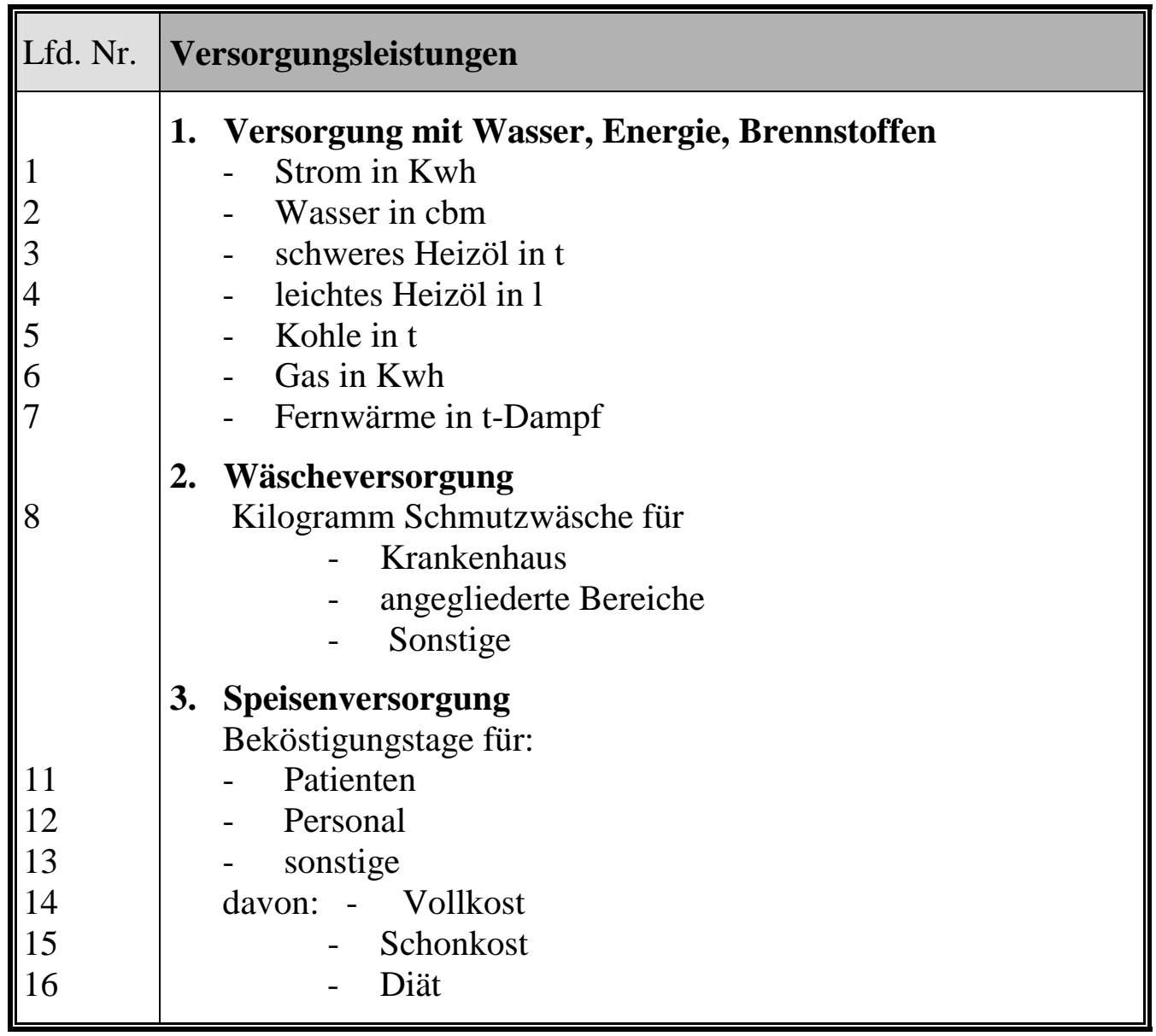

Abb. 30: Versorgungsleistungen im Krankenhaus ${ }^{408}$

Die Statistiken der Leistungen der medizinischen Institutionen sind in der neuen Bundespflegesatzverordnung nicht vorgesehen. ${ }^{409}$ Aber es ist jedoch auf Grund des neuen Vergütungssystems erforderlich, diese Leistungsartenstatistiken zuzuweisen und $\mathrm{zu}$ bewerten. Sie können in getrennter Aufzeichnung für Leistungen der ambulanten und stationären Bereiche erfasst und über die Ziffern nach der GOÄ und dem DKG-NT bewertet werden (Abb. 31).

\footnotetext{
408 Quelle: Teil S5 Anlage 3 zu § 17 Abs. 4 BPflV.

409 In der BPflV a.F. wurde bis 1995 eine Leistungsstatistik für alle medizinischen Institutionen vorgeschrieben. Anlage 2 zur $\S 16$ Abs. 4 BPflV a.F., Teil L2 des Kosten- und Leistungsnachweises.
} 


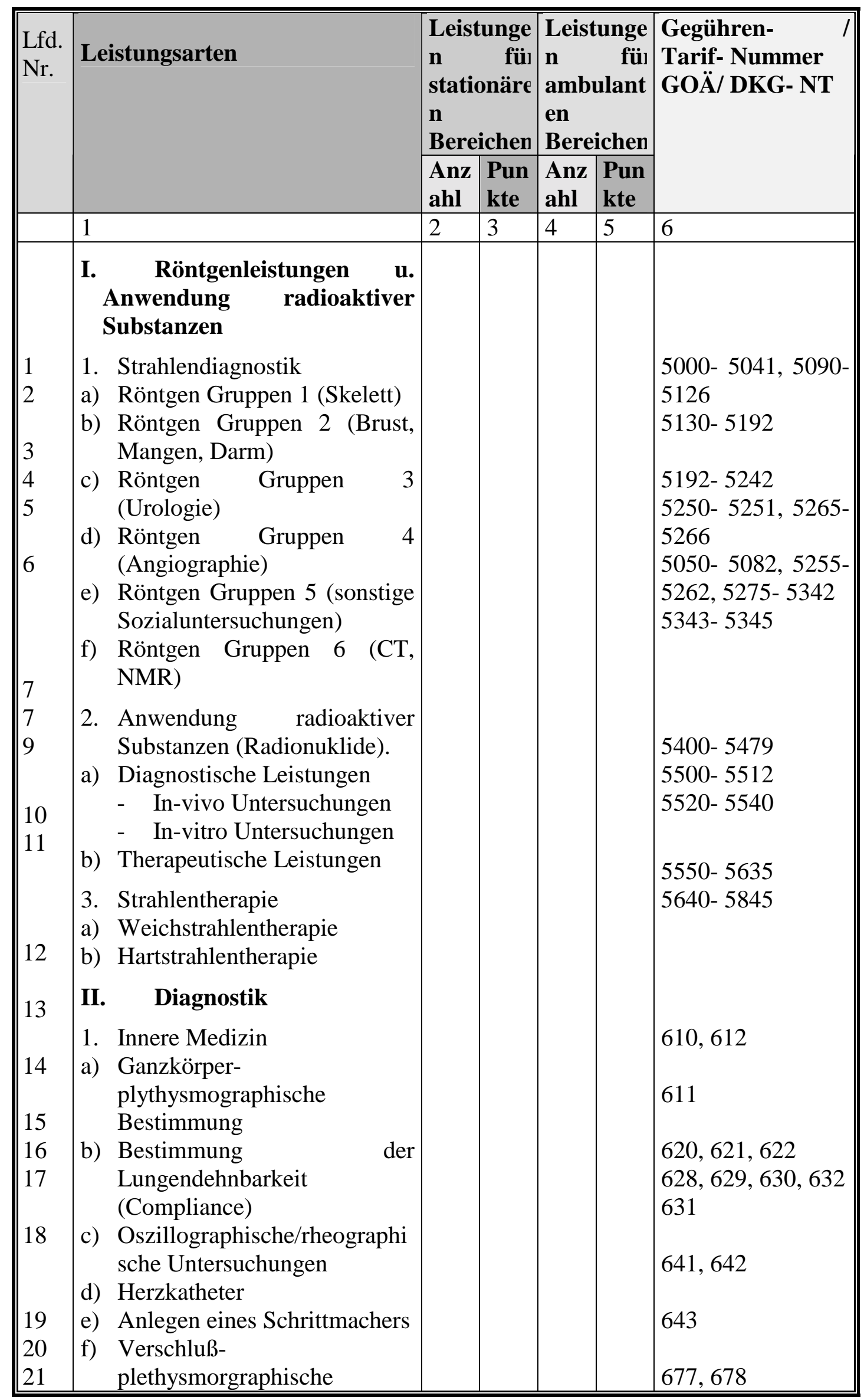




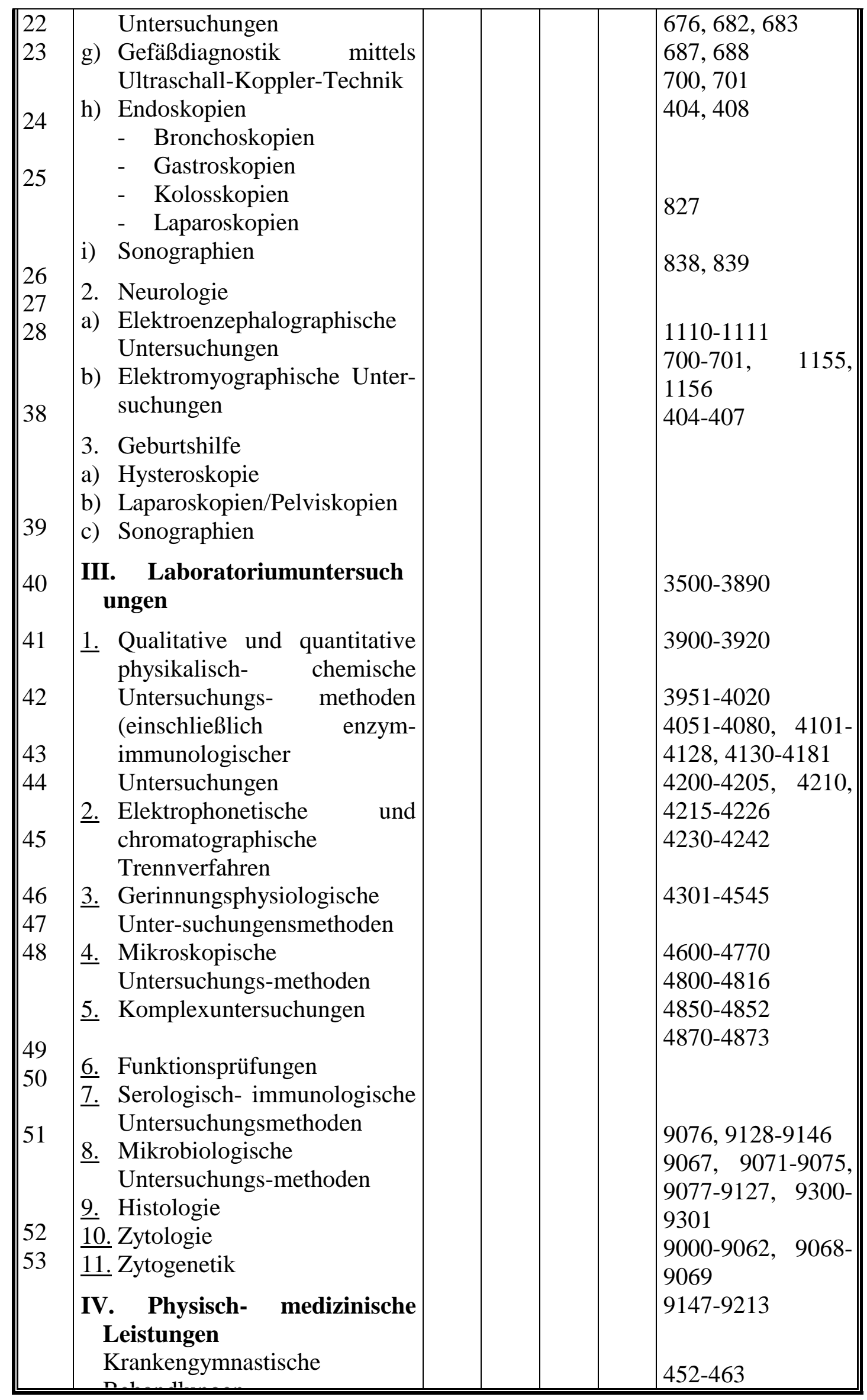




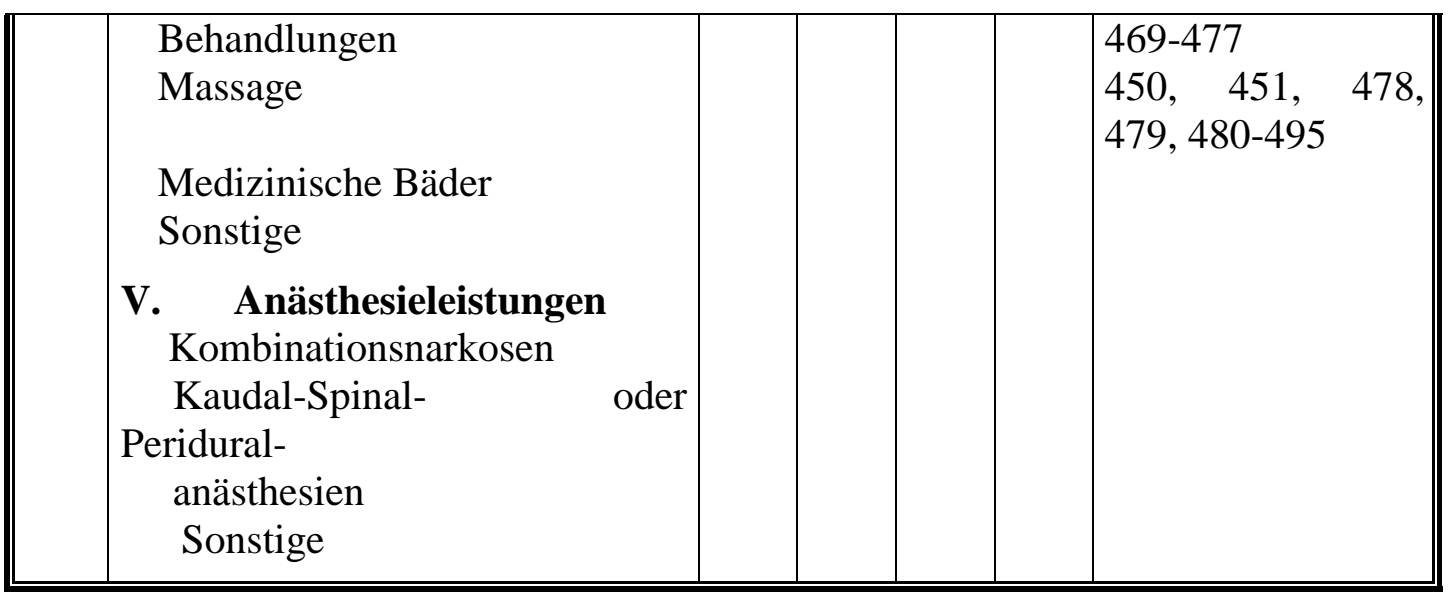

Abb. 31: Leistungsstatistik für medizinische Institutionen. ${ }^{410}$

Weitere Leistungsstatistiken können auch nach den individuellen Anforderungen eines Krankenhauses durchgeführt werden, soweit Informationen (wie z. B. Zentralsterilisations-, Werkstattleistungen oder Krankenhaustransporte) für Zwecke der exakten Kosten- und Leistungsrechnung bzw. der Wirtschaftlichkeitsbeurteilung benötigt werden.

\subsection{Teilgebiete der Kosten- und Leistungsrechnung in syrischen Kranken- häusern}

Zur Erfüllung des Zweckes von homogener Zusammenstellung der Daten über alle Betriebe in allen Wirtschaftsbereichen hat der syrische Gesetzgeber einheitliche Anforderungen an die Teilgebiete der Kosten- und Leistungsrechnung bestimmt, um die Systeme und Verfahren des Rechnungswesens und der Kosten- und Leistungsrechnung zu vereinheitlichen

\subsubsection{Kostenartenrechnung in syrischen Krankenhäusern}

\subsubsection{Definition und Aufgaben der Kostenarten}

Abgesehen von der Notwendigkeit, sowohl für die zentrale Nationalverrechnung als auch für die Kosten- und Leistungsrechnung die Kostenarten-Kontierung vorzunehmen, hat der syrischen Gesetzgeber einen einheitliche Kontenrahmen definieren, der sowohl in der Finanzbuchführung als auch in der Kosten- und

410 In Anlehnung an: Anlage 2 zur $\S 16$ Abs. 4 BPflV a.F., Teil L2 des KLA, und Heintze, J., Kehres, E. (1995), S. 81- 85. 
Leistungsrechnung benutzt werden muss. ${ }^{411}$ Derartige Kostenarten sind auch ausführlich im SERS definiert, womit die Daten, die aus den Rechnungsinstrumente verschiedener Betriebe entnommen sind, transparent zusammengestellt und verglichen werden können. ${ }^{412}$

Im Rahmen der Kosten- und Leistungsrechnung sind die Kosten gemäß dem SERS nach Art der verbrauchten Kostenfaktoren gegliedert. Sie umfassen KostenartenGruppen von Personalkosten, Materialkosten, produktionszweckabhängigen Kosten, Produktionszweck unabhängigen Kosten und Investitionskosten.

In einem Krankenhaus können die Kostenarten-Gruppen von Personalkosten, Materialkosten, Behandlungs- und pflegezweck-abhängige Kosten, Behandlungsund pflegezwecke-unabhängige Kosten und Investitionskosten unterschieden werden.

\subsubsection{Erfassung der Kostenartenrechnung in syrischen Krankenhäusern}

Die im Kontenrahmen des SERS nach Art der verbrauchten Kostenfaktoren gegliederten Kostenarten sind in Kostenartengruppen (Personalkosten, Materialkosten, usw.) differenziert. Jede dieser Kostenartengruppen umfasst Untergruppen (z. B. werden die „Personalkosten“ in Löhne und Gehälter, Prämien und Sozialabgaben unterteilt). Die Kostenartenuntergruppen wiederum sind in detaillierte Kostenarten differenziert(so umfasst bspw. „Prämien“, Wohnen, Essen, usw.) Nach der Mindestanforderung des SERS sollen alle Betriebe ihre Kostenartengruppen und Kostenartenuntergruppen gemäß des vorgeschriebenen Kostenmusters differenzieren. Allerdings dürfen sie die detaillierten Kostenarten nach dem eigenen Bedarf bestimmen.

Analog zur Kontenrahmen des SERS könnten die folgende Kostenarten in einem syrischen Krankenhaus erfasst werden:

- Personalkosten (Kontenartengruppe 31): Das Personal im Krankenhaus ist nach SERS in folgende Kategorien geordnet:

411 Die Kontenrahmen erfassen 9 Kontenklassen. Sie sind u. a. Kontenklassen 01 „Bilanz-Aktiva“, Kontenklassen 02 „Bilanz-Passiva“, Kontenklassen 03 „Kosten“, Kontenklassen 04 „Leistungen“ und Kontenklassen 05 bis 09 „Kostenstellen“.

412 „ 4 Abs. 1 SERS (Übersetzung der Verfaser". . Die Kostenarten sollen nach den Kontenrahmen dieses Gesetzes in den homogenen Gruppen klassifiziert werden. Sie sind zur Erfüllung der Zwecke der Nationalverrechnung und der interne Verrechnung geeignet ." 
a. Personal der „Produktionsabteilungen“, Ärzte und Pflegern der Pflegefachbereiche,

b. Personal der „Produktionsentlastungen“, Ärzte, Pflegern sowie Funktionspersonal der medizinischen Institutionen sowie Versorgungseinrichtungen und

c. Personal der Wirtschafts- und Verwaltungsbereiche.

Dienstarten der Personalkosten und ihre Struktur im syrischen Krankenhaus sind in Tab. 17 dargestellt:

\begin{tabular}{|l|l||}
\hline Dienstarten der Personalkosten & Struktur \\
\hline Ärztlicher Dienst der Pflegefachbereiche & $26 \%$ \\
\hline Pflegedienst der Pflegefachbereiche & $19 \%$ \\
\hline Ärztlicher Dienst der Institutionen & $7 \%$ \\
\hline Pflegedienst der Institutionen & $11 \%$ \\
\hline Medizinisch- technischer- und Funktionsdienst & $9 \%$ \\
\hline Wirtschafts- und Versorgungsdienst & $4 \%$ \\
\hline Technischer Dienst & $8 \%$ \\
\hline Klinisches Hauspersonal und Verwaltungsdienst & $10 \%$ \\
\hline Personal der Ausbildungsstätten & $4 \%$ \\
\hline Sonstiges Personal & $2 \%$ \\
\hline & $100 \%$ \\
\hline
\end{tabular}

Tab. 17: Dienstarten der Personalkosten. ${ }^{413}$

Die Personalkosten erfassen nach dem SERS Löhne und Gehälter, Prämieren und Gesetzliche Sozialabgaben.

Löhne und Gehälter sind monetäre Entllohnungen für das Personal. Dabei soll für Informierenszwecke zwischen Löhnen und Gehältern für Werk-, Urlaubsund Krankheitstage unterschieden werden, ebenso Löhne und Gehälter für ausländisches Personal.

Die Prämien sind Entlohnungen für das Personal in nicht-monitärer Formen. Sie umfassen Wohnungen, Essen, Krankenversicherungen Kleidungen, und

413 In Anlehnung an: Universitätsklinik Damaskus (1998): Jahres Bericht 1997, Damaskus 4/ 1998, S. 10 und 15. 
Transporte, die vom Arbeitgeber entrichtet und extra (nicht nach dem Betriebsbedarf) an das Personal kostenlos ausgegeben werden.

Gesetzliche Sozialabgaben beinhalten den Anteil der Rente und Sozialversicherungen, der vom Arbeitgeber abgetragen werden muss.

- Materialkosten (Kontenarten-gruppen 32): Diese erfassen die Kosten aller Materialien, die im Behandlungs- und Pflegeprozess in Pflegefachbereichen oder medizinischen Institutionen benutzt werden müssen. Dazu zählen der medizinische Bedarf, Lebensmittel, Energie und Brennstoffe, Bekleidung, technischer Bedarf und wirtschaftlicher Bedarf.

- Behandlungs- und Pflegezwecke abhängige-Kosten (Kontenartengruppe 33): Hier handelt es sich um die im Behandlungs- und Pflegeprozess benötigten Kosten, die nicht vom Krankenhaus-Personal oder -Einrichtungen, sondern durch fremde Einrichtungen anfallen werden. Wie Instandhaltung, Kosten der Leistungen bei Fremden, Transportkosten, Wasser und Strom, Post- und Telefonkosten.

- (Kontenartengruppe 35): Diese Gruppe erfasst diejenigen Kosten, die nicht direkt von Behandlungs- und Pflegezwecke abhängig sind. Sie sind von den Kostenkomponente nach dem wertmäßigen Gesichtspunkt. Hier zu zählen Kosten wie Abschreibungen, Miete, Kalkulatorische Miete, Zinsen und Kalkulatorische Zinsen.

In Tab 18 sind die Kostenarten (Kontenarten-gruppen und Kostenarten-untergruppen) im syrischen Krankenhaus dargestellt.

\begin{tabular}{|c|c|}
\hline Kontenartengruppen & Kostenarten-Untergruppen \\
\hline \multirow[t]{3}{*}{31 Personalkosten } & 311 Löhne und Gehälter \\
\hline & 312 Prämien \\
\hline & 312 Gesetzliche Sozialabgaben \\
\hline \multirow[t]{4}{*}{32 Materialkosten } & 321 Medizinische Bedarfs \\
\hline & 322 Lebensmittel \\
\hline & 323 Energie und Brennstoff \\
\hline & 324 Bekleidung \\
\hline
\end{tabular}




\begin{tabular}{|c|c|c|}
\hline & & 325 Technische Bedarfs \\
\hline & & 327 Wirtschaftliche Bedarfs \\
\hline 33 & Behandlungs- $\quad$ und & 332 Instandhaltung \\
\hline & $\begin{array}{l}\text { Pflegezweck-abhängigen } \\
\text { Kosten }\end{array}$ & 333 Kosten der Fremdleistungen \\
\hline & & 336 Transportkosten \\
\hline & & 337 Wasser und Strom \\
\hline & & 338 Post- und Telefonkosten \\
\hline 34 & & 341 Apotheke \\
\hline & Finanzierungskosten und & 351 Abschreibungen \\
\hline & Sonstige Kosten & 352 Miete \\
\hline & & 353 Kalkulatorische Miete \\
\hline & & 355 Zinsen \\
\hline & & 366 Kalkulatorische Zinsen \\
\hline
\end{tabular}

Tab. 18: Kostenarten im syrischen Krankenhaus.

\subsubsection{Kostenstellenrechnung in syrischen Krankenhäusern}

\subsubsection{Definition und Aufgaben der Kostenstellenrechnung in syrischen}

Krankenhäusern

Die Einführung der Kostenstellen in syrischen Krankenhäusern ist gesetzlich im SERS vorgesehen. In dem Muster-Kontenrahmen des SERS sind die Kostenstellen, die von allen Betrieben benutzt werden müssen.

Die Kostenstellen in einem Betrieb werden in Produktions-, Produktionshilfs-, Vertriebs- und Verwaltungskostenstellen unterschieden.

Eine direkte Ermittlung aller Kostenarten hat das SERS als Aufgabe der Kostenstellenrechnung genannt. Da die gesamten Betriebsbereiche in Kostenstellen aufgeteilt werden, können alle Kosten als direkte Kosten an die Kostenstellen verrechnet werden. ${ }^{414}$ Weitere Aufgaben der Kostenstellen sind die Kostenkontrolle

${ }^{414}$ Eine besondere Definition der direkten Kosten ist vom SERS verwendet worden, nach der sie an bestimmte Kostenstellen, aber nicht unbedingt nach Leistungseinheiten verrechnet werden müssen. Z. B. ist der Gehalt des Betriebsleiters direkte Kosten der Kostenstellen Verwaltung, obwohl er keine direkte Kosten aus dem Sinne der Leistungserstellung. 
und Kostenplanung, da diese Kostenstellen gleichzeitig einzelne Verantwortungsbereiche darstellen.

\subsubsection{Bildung der Kostenstellenrechnung in den syrischen Krankenhäusern}

Das SERS hat die Kostenstellen nach dem Kriterium der Funktionsbereiche eines Industriebetriebs gebildet, womit die Kostenstellen in einem Betrieb nach den Produktion-, Produkthilfs-, Vertriebs- und Verwaltungsbereichen bestimmt werden. Analog dazu sollen Kostenstellen eines syrischen Krankenhauses nach seinen Funktionsbereichen gebildet werden. D. h. die Kostenstellengruppen sollen nach Behandlungs- und Pflegebereichen, medizinische Institutionen, Versorgungseinrichtungen und Verwaltungsbereichen gebildet werden. ${ }^{415}$

Nach dem Abrechnungsverfahren der Kosten- und Leistungsrechnung des SERS sind die Behandlungs- und Pflegekostenstellen als Hauptkostenstellen, medizinische Institutionen und Versorgungskostenstellen als Hilfskostenstellen und Verwaltungskostenstellen als Nebenkostenstellen zu behandeln.

Die Kostenstellen eines syrischen Krankenhauses können wie folgt dargestellt werden (Tab. 19)

\begin{tabular}{||l|l|l||}
\hline $\begin{array}{l}\text { Kosten- } \\
\text { stellen Nr. }\end{array}$ & Kostenstellenbezeichnung & Kostenstellen-Kategorie \\
\hline $\mathbf{5}$ & Pflegekostenstellen & Hauptkostenstellen \\
501 & Kinderkardiologie & \\
502 & Allgemeine Nephrologie & \\
503 & Kardiologie & \\
504 & Lungen u. Bronchialheilkunde & \\
505 & Pädiatrie & \\
506 & Geriatrie & \\
507 & Hämatologie u. künst. Onkologie & \\
508 & Infektion & \\
509 & Gastroenterologie & \\
510 & Allgemeine Chirurgie & \\
\hline
\end{tabular}

415 Bislang gibt es in den Krankenhäusern keine Vertriebseinrichtungen, es kann sich allerdings um „Public Relation“ handelt. 


\begin{tabular}{||l|l|l||}
\hline 511 & Unfallchirurgie & \\
512 & Kinderchirurgie & \\
513 & Endoprothetik & \\
514 & Thoraxchirurgie & \\
515 & Handchirurgie & \\
516 & Gefäßchirurgie & \\
\hline $\mathbf{6}$ & medizinische Institutionen und & Hilfskostenstellen \\
& Versorgungskostenstellen & \\
601 & Röntgendiagnostik und -therapie & \\
602 & Nukleardiagnostik und -therapie & \\
603 & Laboratorien & \\
604 & Funktionsdiagnostik & \\
605 & Sonstige diagnos. Einrichtungen & \\
606 & Anästhesie & \\
607 & OP- Einrichtungen & \\
608 & Physikalische Therapie & \\
609 & Kreißzimmer & \\
610 & Pathologie & \\
611 & Ambulanzen & \\
612 & Sonst. therap. Einrichtungen & \\
620 & Speisenversorgung & \\
621 & Wäscheversorgung & \\
622 & Zentr. Reinigungsdienst & \\
623 & Innerbetriebl. Transport & \\
624 & Zentrale Sterilisation & \\
\hline $\mathbf{7}$ & Vertriebskostenstellen & \\
701 & P. R & \\
\hline $\mathbf{8}$ & Verwaltungs-Kostenstellen & \\
801 & Verwaltung & \\
\hline $\mathbf{9}$ & Investitions-Kostenstellen & \\
901 & je Investitionsobjekt & \\
\hline
\end{tabular}

Tab. 19: Kostenstellen eines syrischen Krankenhauses.

${ }^{416}$ Bei Investitionskostenstelle handelt es sich nicht um eine Kostenstelle in Sinne der Kosten- und Leistungsrechnung, sonder um neues Investitionsobjekt (Vermögen). Das SERS hat diese Kostenstellen erstellt um alle Kosten, die zu einem neuen Vermögen, wie z.B. neue Abteilung oder neue Gerät zählen, separat ermittelt werden können. 


\subsubsection{Durchführung der Kostenstellenrechnung in syrischen Kranken- häusern}

Nach der Ermittlung der Kosten in den gebildeten Kostenstellen, werden die Kosten der Produktion-, Produktionshilfs-kostenstellen, im Rahmen der Kostenstellenrechnung durchgeführt werden, da die Durchführung der Kostenrechnung ist grundsätzlich als Teilkostenrechnung zu konzipieren.

Analog dazu sind die im syrischen Krankenhaus angefallenen Kosten der Behandlungs- und Pflegebekostenstellen 501-516 sowie der MedizinischeInstitutionen-Kostenstellen 601-612 und Versorgungskostenstellen 620-624 im Rahmen der Kostenstellenrechnung zu behandeln.

Hingegen müssen die Kosten der Vertriebskostenstelle 701 und Verwaltungskostenstelle 801 außerhalb der Kostenrechnung zu Verrechnet werden, und zwar in der Ergebnisrechnung.

Und da die Investitionskostenstellen 9 keine Kostenstellen in Sinne der Kosten- und Leistungsrechnung darstellen, dürfen sie weder in die Kostenstellenrechnung noch in die Ergebnisrechnung eingehen. Sie sollen als Vermögenswert in der Bilanz ankommen.

Bei der Durchführung der Kostenstellenrechnung stellen die Behandlungs- und Pflegekostenstellen 501-516 Endkostenstellen und die medizinische-InstitutionenKostenstellen601-612 sowie die Versorgungskostenstellen 620-624 Vorkostenstellen dar. Die Kosten der Behandlungs- und Pflegekostenstellen werden direkt verrechnet sind, während die Kosten der Medizinische-Institutionen-Kostenstellen und Versorgungskostenstellen weiter auf die Behandlungs- und Pflegekostenstellen verteilt werden sollen. Als Grund der Verteilung hat das SERS vorgeschrieben, dass die Kosten der Produktionshilfskostenstellen nach dem tatsächlichen beanspruchten Abgang auf die Produktionskostenstellen verteilt werden müssen. Dies betrifft auch die Krankenhäuser, weil die Kosten der Medizinische-InstitutionenKostenstellen als innerbetrieblichen Leistungen und die Kosten der Versorgungskostenstellen möglichst auf Grundlage vom tatsächlichen Verbrauch auf die Behandlungs- und Pflegekostenstellen und auf Grundlage von Verteilungsschlüsseln (wenn keine tatsächliche Relation möglich ist) verteilt werden. 


\subsubsection{Kostenträgerrechnung in syrischen Krankenhäusern}

\subsubsection{Kostenträgerstückrechnung in den syrischen Krankenhäusern}

Der syrische Gesetzgeber hat keine Kostenträgerstückrechnung vorgesehen. Nach dem SERS sollen in der Kostenstellenrechnung verrechnete Kosten jeder Kostenstelle auf die den in der selben Kostenstelle erstellten Leistungseinheiten verteilt werden. ${ }^{417}$

Analog dazu sollen in den syrischen Krankenhäuser nach SERS die Kosten jeder von den Behandlungs- und Pflegebekostenstellen auf den in derselben Kostenstelle erstellten Leistungseinheiten verteilt werden.

Die Problematik hierbei ist die Verwendung des Divisionsverfahrens, da die Leistungen des Krankenhauses, aber auch einer Krankenhaus-Kostenstelle heterogen sind. Damit ist eine Bestimmung der Leistungseinheiten eines Krankenhauses oder einer Krankenhaus-Kostenstelle schwierig. Während in der Praxis eines staatlichen Krankenhauses in Syrien für Statistikzwecke die Gesamtkosten einer Kostenstellen auf „Patient/Tag“ als Leistungseinheit dividiert werden, wird hingegen in der Praxis der privaten Krankenhäuser jeder Patient (Einzelfall) als Kalkulationsobjekt behandelt.

\subsubsection{Kostenträgerzeitrechnung in syrischen Krankenhäusern}

Die Kostenträgerzeitrechnung ist nach SERS im Rahmen der Ergebnisrechnung (Erfolgsrechnung) zu betrachten. Das SERS hat in seinem Kontenrahmen (Kontenklasse 4) die verschiedenen Erlöse des Betriebs bestimmt, wobei es zwischen Erlöse aus Betriebsleistungen und anderen weiteren Leistungen unterscheidet.

Das SERS hat dafür gesorgt, dass die Kostenträgerzeitrechnung nach dem Gesamtkostenverfahren durchgeführt werden kann. Somit werden die nach

417 SERS hat Leistungseinheit als Messgröße definiert, die die Leistung einer Kostenstelle, einer Produktionsstufe oder des Betriebs determinieren. Sie kann nach Stückzahl, Gewicht oder Länge in Fall der Konformität der Leistungen ermittelt werden. Und wenn die Leistungen nicht identisch sind, können sie nach einem von den Kostenfaktoren (z. B. Arbeitsstunden) oder einer annähernden Messgröße ermittelt werden. In der Praxis der syrischen Krankenhäuser ist eine Meßgröße von Patienten/Tag zur Anweisung der Krankenhausleistungen zu benutzen. Vgl. §17 Abs. 1 SERS. 
Kostenarten gegliederten Gesamtkosten und Gesamterlöse eines Betriebs entsprechend der Gewinn- und Verlustrechnung gegenübergestellt.

Entsprechend können in einem syrischen Krankenhauses für Zweck der kurzfristigen Erfolgsrechnung sich diejenigen Erlösarten ergeben, die in Tab. 20 dargestellt.

\begin{tabular}{|c|c|}
\hline Erlösarten-Gruppen & Erlösarten-Untergruppen \\
\hline 41 Erlöse aus Krankenhausleistungen & $\begin{array}{l}410 \text { Erlöse der Kinderkardiologie } \\
411 \text { Erlöse der Allgemeine-Nephrologie } \\
412 \text { Erlöse der Lungen u. } \\
\text { Bronchialheilkunde } \\
413 \text { Erlöse der Pädiatrie } \\
414 \text { Erlöse der Chirurgie } \\
415 \text { Erlöse der Endoprothetik }\end{array}$ \\
\hline 42 Erlöse der Wahlleistungen. & $\begin{array}{l}421 \text { Erlöse der ärztlichen Wahlleistungen } \\
422 \text { Erlöse der sonstigen Wahlleistungen } \\
\text { (Telefon, TV, Unterkunft) }\end{array}$ \\
\hline 43 Erlöse aus Leistungen für Dritten & $\begin{array}{l}431 \text { Vergütung der Leistungen für andren } \\
\text { Krankenhäuser } \\
431 \quad \text { Erlöse der vergüteten } \\
\text { wissenschaftlichen Untersuchungen }\end{array}$ \\
\hline 44 Erlöse aus verkauften Waren & 441 Erlöse der eigenen Apotheke \\
\hline 46 Ausschüsse & $\begin{array}{l}451 \text { staatliche Ausschüsse } \\
452 \text { Ausschüsse von Kommunen oder } \\
\text { Organisationen }\end{array}$ \\
\hline 49 sonstige Erlöse & 491 Erlöse aus Sonstigem \\
\hline
\end{tabular}

Tab 20: Erlösarten im syrischen Krankenhaus

\section{Systeme der Kosten- und Leistungsrechnung im Krankenhaus}

Alle für erwerbswirtschaftliche Sach- oder Dienstleistungsbetriebe entwickelten Kostenrechnungssysteme stehen auch für Krankenhausbetriebe zur Verfügung. Das Kostenrechnungssystem dient der orientierten Zurechnung von Kosten zu bestimmten Bezugsgrößen. In dieser allgemeinen Definition eines Kostenrechnungssystems werden die Probleme offensichtlich, die bei der Unterscheidung von 
Kostenrechnungssystemen auftreten. ${ }^{418}$ Wie Kosten $\mathrm{zu}$ definieren sind, nach welchen Prinzipien diese Kosten $\mathrm{zu}$ verteilen sind und wie Rechenregeln für ein Kostenrechnungssystem ausgestaltet werden sollen, sind einige der offenen Probleme einer Theorie der Krankenhaus-Kostenrechnung.

\subsection{Allgemeine Betrachtungen an Systeme der Kosten- und Leistungs- rechnung im Krankenhaus}

Kostenrechnungssysteme lassen sich nach verschiedenen Kriterien bilden. In der Literatur und praktischen Anwendung werden Kostenrechnungssysteme vor allem nach zwei Kriterien unterschieden:

- $\quad$ dem Zeitbezug der Kosten und

- $\quad$ dem Umfang der Kostenzurechnung auf eine Leistungseinheit.

Die Kombinationsmöglichkeiten, die sich daraus ergeben, sind in Abb. 32 dargestellt.

\begin{tabular}{|c|c|c|c|}
\hline $\begin{array}{l}\text { Kostenrechnungs- } \\
\text { systeme }\end{array}$ & Istkostenrechung & $\begin{array}{l}\text { Normalkosten- } \\
\text { rechnung }\end{array}$ & $\begin{array}{l}\text { Plankostenrechn } \\
\text { ung }\end{array}$ \\
\hline $\begin{array}{l}\text { Vollkostenrechnu } \\
\text { ng }\end{array}$ & $\begin{array}{l}\text { Vollkostenrechnun } \\
\mathrm{g} \quad \text { auf } \\
\text { Istkostenbasis }\end{array}$ & $\begin{array}{l}\text { Vollkostenrechnun } \\
\text { g auf } \\
\text { Normalkostenbasis }\end{array}$ & $\begin{array}{l}\text { Vollkostenrechnu } \\
\text { ng auf } \\
\text { Plankostenbasis }\end{array}$ \\
\hline $\begin{array}{l}\text { Teilkostenrechnu } \\
\text { ng }\end{array}$ & $\begin{array}{l}\text { Teilkostenrechnun } \\
\mathrm{g} \quad \text { auf } \\
\text { Istkostenbasis }\end{array}$ & $\begin{array}{l}\text { Teilkostenrechnun } \\
\mathrm{g} \quad \text { auf } \\
\text { Normalkostenbasis }\end{array}$ & $\begin{array}{l}\text { Teilkostenrechnun } \\
\mathrm{g} \text { auf } \\
\text { Plankostenbasis }\end{array}$ \\
\hline
\end{tabular}

Abb. 32: Systeme der Kostenrechnung im Überblick ${ }^{419}$

Die Auswahl des für einen bestimmten Betrieb optimalen Kostenrechnungssystems orientiert sich am Rechnungszweck, den technisch- organisatorischen Betriebsgegebenheiten sowie den rechtlichen Rahmenbedingungen. Es sollte sich aber auch mit der Entwicklung des Betriebsprozesses entwickeln. ${ }^{420}$

\footnotetext{
418 Vgl. Leonhardt, J. (1988), S. 19.

419 In Anlehnung an Steger, J: Kosten- und Leistungsrechnung, München, 1996, S. 130.

${ }^{420}$ Toso, M. E. (1994): The Value of a Cost Accounting System, in: Finkler, S. A. (Edit.),Issues in Cost Accounting for Health Care Organisations, New York, 1994, S. 6.
} 
Um eine Empfehlung für ein bestimmtes Kostenrechnungssystem für Krankenhausbetriebe geben zu können, müssen die Fragen wie z. B. nach den Adressaten, dem Zwecke und dem Rhythmus der Erstellung sowie der benötigten Informationen beantwortet werden. ${ }^{421}$ Soweit die Kosten- und Leistungsrechnung mehrere Rechnungszwecke zu erfüllen hat, kann das auch die Verwendung unterschiedlicher Wertansätze für die eingesetzten Produktionsfaktoren bedeuten. Dies gilt auch für die in Krankenhäusern anzuwendenden Kostenrechnungssysteme. ${ }^{422}$ Dabei muss jedes Krankenhaus eine individuelle Entscheidung treffen, welches Kostenrechnungssystem nach Studien und Erfahrungen zu verwenden ist. ${ }^{423}$

\subsubsection{Zeitbezogene Systeme}

Nach dem zeitlichen Bezug der verrechneten Kosten (vergangenheitsbezogene oder zukunftsorientierte Kosten) sind Ist-, Normal- und Plankostenrechnung zu unterscheiden. Die Verwendung von Kosten mit unterschiedlichem Zeitbezug dient neben der Vereinfachung des Abrechnungsvorgangs hauptsächlich der Verbesserung der Kostenkontrolle. So hat sich aus der Istkostenrechnung erst die Normalkostenrechnung und schließlich die Plankostenrechnung entwickelt. Die zeitbezogenen Kostenrechnungssysteme stellen den Istkosten der Abrechnungsperiode als Kontrollmaßstab jeweils verschiedene Kosten gegenüber, so dass sich verschiedene Vergleiche ergeben. ${ }^{424}$

\subsubsection{Istkostenrechnung}

In der Istkostenrechnung werden die tatsächlich angefallenen Kosten verrechnet. Die Kostenrechnung wird am Ende einer Rechnungsperiode erstellt und dient als Ergebnisrechnung dem Ausweis des tatsächlichen Erfolgs sowie als Nachkalkulation der nachträglichen Ermittlung der Selbstkosten der einzelnen Kranken-

\footnotetext{
${ }^{421}$ Vgl. Leonhardt, J. (1988), S. 24.

${ }^{422}$ Vgl. Hentze, J., Kehres, E. (1995), S. 43

${ }^{423}$ Vgl. Davis Weintraub, L., Dube, R. J. (1994): Alternative Costing Methods in Health Care, in: Finkler, S. A. (Edit.), Issues in Cost Accounting for Health Care Organisations, New York, 1994 , S. 118.

${ }^{424}$ Vgl. Haberstock, L. (1998), S. 172.
} 
hausleistung. Hauptzweck der Istkostenrechnung ist die Feststellung der effektiven Kosten einer Kostenstelle oder eines Kostenträgers. ${ }^{425}$

Aus dem System der Istkostenrechnung ergibt sich die Möglichkeit des Zeit- und Betriesvergleichs. Im Zeitvergleich (Ist- Ist- Vergleiche) werden Istkosten zweier verschiedener Periode verglichen. Der Aussagewert ist gering, da die Kosten der Vergleichsperiode bereits selbst durch Zufälligkeiten und Unwirtschaftlichkeit überhöht sein können, ohne dass diese feststellbar sind.

Im Betriebsvergleich können Istkosten derselben Periode, aber verschiedener Betriebe vergleicht werden. Dabei tritt das Problem der Vergleichbarkeit der Betriebe auf. Wenn als Vergleichsmaßstab Durchschnittszahlen der gesamten Branche verwendet werden, ist die Aussagekraft dadurch eingeschränkt, dass der fiktive Durchschnittsbetrieb eben doch nicht mit dem Einzelbetrieb vergleichbar ist. $^{426}$

\subsubsection{Normalkostenrechnung}

Der Normalkostenrechnung liegen Durchschnittswerte der Vergangenheit zugrunde. Dabei kann die Durchschnittsbildung für die Preise und Mengen erfolgen. Die Kostennormalisierung erleichtert und beschleunigt die Abrechnungsarbeit und schaltet die Zufallsschwankung der Kosteneinflussfaktoren aus. Die Normalkostenrechnung - im Krankenhauswesen der USA mittlerweile der Regelfall - arbeitet mit Kostenstandards. Für die einzelnen Kostenarten werden feste Verrechnungspreise verwendet. ${ }^{427}$

Die Normalkostenrechnung kann sowohl als starre als auch als flexible Rechnung durchgeführt werden. Die starre Normalkostenrechnung weist die Kosten für eine geplante Beschäftigung aus und trennt die geplanten Kosten nicht in fixe und variable Kosten. In der flexiblen Normalkostenrechnung werden die Einflüsse von Beschäftigungsschwankungen auf die Kostenhöhe berücksichtigen, während sie als

\footnotetext{
${ }^{425}$ Vgl. Hentze, J. Kehres, E. (1995), S. 29.

${ }^{426}$ Vgl. Freidank, C. (1994). S. 187-188.

${ }^{427}$ Vgl. Hildbrand, R. (1988), S. 440.
} 
starre Normalkostenrechnung einfacher ist, ermöglicht die flexible Normalkostenrechnung eine verfeinerte Kostenkontrolle. ${ }^{428}$

Vorteile der Normalkostenrechnung ergeben sich einerseits durch die Vereinfachung der Betriebsabrechnung und der Vorkalkulation. Anderseits bietet die Normalkostenrechnung Ansatzpunkte für eine Wirtschaftlichkeitskontrolle, da sie einen Normal-Ist-Vergleich ermöglicht, indem die Abweichung zwischen Normalund Istkosten durch den Vergleich der Istkosten mit dem aus dem Durchschnitt der Istkosten mehrerer vergangener Perioden gewonnenen Normalkosten analysiert werden. Zwar sind Zufälligkeiten aus den Normalkosten weitgehend eliminiert, sie können aber weiterhin nicht feststellbare Unwirtschaftlichkeiten enthalten und sind damit als Maßstab für die Kostenkontrolle ungeeignet. ${ }^{429}$

\subsubsection{Plankostenrechnung}

Die Plankostenrechnung begegnet den Mängeln der Ist-Kostenrechnung. Sie hat das Ziel, die Zufallsschwankungen im Kostenanfall $\mathrm{zu}$ isolieren und die hauptsächlichen Verursachungsgrößen im Güter- und Leistungsverzehr aufzudecken. ${ }^{430}$

Mit der Anwendung eines Kontroll- und Steuerungsinstrumentes erfüllt die Kostenrechnung nur dann die ihr gestellte Aufgabe, wenn sie sich nicht nur auf die Analyse der Vergangenheit beschränkt, sondern zusätzlich eine Kostenplanung einbezieht. Dieser Forderung wird nur eine zukunftsorientierte Plankostenrechnung gerecht. Im Gegensatz zur vergangenheitsorientierten Istkostenrechnung ist die Plankostenrechnung ein zukunftsorientiertes Kostenrechnungssystem, das im Rahmen von Abweichungsanalysen Kostenabweichungen zwischen geplanten und tatsächlich entstandenen Kosten ermittelt und analysiert. ${ }^{431}$ Aus der Analyse der Abweichungen werden Erkenntnisse für die Steuerung und Rationalisierung des Krankenhausprozesses gewonnen. ${ }^{432}$ Da der Gewinn als „Meßlatte“ für den Erfolg

\footnotetext{
${ }^{428}$ Vgl. Freidank, C, (1994), S. 195.

${ }^{429}$ Vgl. Haberstock, L. (1998), S. 174-175.

${ }^{430}$ Vgl. Rippel, W. H., Posinisky, R. (1987), S. 347.

431 Plankosten sind die im Voraus methodisch bei ordnungsgemäßem Betriebsablauf als erreichbar betrachteten, mit Planpreisen (soweit bestimmbar) bewerteten leistungsbezogenen Kostengüterverbräuche, die Norm- und Vorgabecharakter besitzen. Vgl. Hummel, S., Männel, W. (1986), S. 47.

${ }^{432}$ Vgl. Hentze, J., Kehres, E. (1995), S. 21-40.
} 
im Krankenhaus weitgehend ausscheidet, ist die Budgetierung mit Plan- IstVergleich wohl das einzige Mittel, das nicht nur eine Dokumentation der Kosten, sondern auch deren Beeinflussung ermöglicht. ${ }^{433}$

Die flexible Plankostenrechnung verlangt darüber hinaus die Beurteilung der Wirtschaftlichkeit durch einen Vergleich der Soll- und Istkosten. Die Sollkosten setzen sich additiv zusammen aus den geplanten fixen Kosten und den auf die IstBeschäftigung umgerechneten variablen Kosten, die als proportional angenommen werden. Dabei werden die aus den Plankosten einer Periode abgeleiteten Sollkosten mit den Istkosten derselben Periode verglichen. ${ }^{434}$ Durch den Vergleich von Sollund Istkosten ist eine wirksame Kostenkontrolle nach Kostenstellen und Kostenarten möglich. Dabei werden die Kostenabweichungen in Preisabweichungen, Verbrauchsabweichungen und Beschäftigungsabweichungen aufgespalten. ${ }^{435}$ Somit ist eine wichtige Zielsetzung der flexiblen Plankostenrechnung die Intensivierung der Wirtschaftlichkeitskontrolle. Eine flexible Plankostenrechnung ist um so wichtiger, je höher der Anteil der variablen Kosten an den Gesamtkosten ist. Sie ist daher im Krankenhaus wegen des dort zumeist sehr hohen Fixkostenblocks nur in Teilbereichen sinnvoll. ${ }^{436}$

\subsubsection{Zurechnungsbezogene Systeme}

Unabhängig davon, ob in einer Kostenrechnung Ist-, Normal- oder Plankosten verrechnet werden, können entweder alle anfallenden Kosten oder nur ein Teil der Kosten auf Kalkulationsobjekte, insbesondere Kostenträger, verrechnet werden. Danach sind Vollkostenrechnung und Teilkostenrechnung zu unterscheiden.

\footnotetext{
${ }^{433}$ Vgl. Hildbrand, R. (1988), S. 438.

${ }^{434}$ Vgl. Schlüchtermann, J., Gorschlüter, P. (1996), S. 100.

${ }^{435}$ Vgl. Hentze, J., Kehres, E. (1995), S. 185-192.

${ }^{436}$ Vgl. Hildbrand, R. (1988), S. 442.
} 


\subsubsection{Vollkostenrechnung}

In der traditionellen Vollkostenrechnung werden sämtlichen Kosten auf Leistungseinheiten zugerechnet (ohne Auflösung). Dabei werden die Gemeinkosten aufgeteilt (geschlüsselt) und die fixen Kosten künstlich proportionalisiert. ${ }^{437}$

In der modifizierten Vollkostenrechnung werden die Kosten aufgelöst. Grundlage ist die Trennung zwischen fixen und variablen Kostenbestandteilen unter dem Aspekt einer veränderten Beschäftigung. Es wird untersucht, ob die betrachteten Kostengröße bei einer Veränderung der Beschäftigungsgröße einen konstanten Verlauf aufweist und damit als fixe Kosten verhält oder ob bei einem veränderlichen Kostenverlauf variable Kosten vorliegen. ${ }^{438}$

Der Nachteil der Vollkostenrechnung besteht darin, dass hier Kosten auf Kostenträger verrechnet werden, die nach dem Verursachungsprinzip nicht zurechenbar sind. Bei dieser Kostenverrechnung ist das Verrechnungsprinzip durch das Durchschnittsprinzip bzw. das Kostentragfähigkeitsprinzip zu ergänzen. ${ }^{439}$

Darüber hinaus muss aus den zusätzlich aus Sicht der betriebsinternen Entscheidungsunterstützung abgeleiteten Aufgaben einer krankenhausspezifischen Kostenund Leistungsrechnung die Erkenntnis gewonnen werden, dass die alleinige Installation eines Kostenrechnungssystems auf Vollkostenbasis nicht ausreicht, um für eine langfristige Existenzsicherung des Krankenhauses detaillierte Informationen $\mathrm{zu}$ liefern. ${ }^{440}$ Damit wird die parallele Installation eines Teilkostenrechnungssystems erforderlich.

\footnotetext{
${ }_{437}$ Vgl. Bopp, M., Hohenbild, R. (1984) S. 52-55.

${ }^{438}$ Vgl. Koch, J. (1998), S. 143.

${ }^{439}$ Nach dem Verursachungsprinzip dürfen nur die Kosten dem einzelnen Kostenträger zugerechnet werden, die dieser verursacht hat. Das Verursachungsprinzip kann demzufolge bei der Verrechnung der Fixkosten in der Kostenträgerrechnung nicht eingehalten werden, da nur die variablen Kosten verursachungsgerecht zugeordnet werden können. Im Durchschnittsprinzip geht es nicht darum, welche Kosten durch welche Leistungen verursacht werden, sondern welche Kosten im Durchschnitt auf welchen Kostenträger bzw. welches Bezugsobjekt entfallen. Im Falle eines Einproduktbetriebes werden alle Fixkosten durch die Anzahl der Leistungen dividiert. Bei der Verteilung der nicht verursachungsgemäß zurechenbaren Kosten, also im Wesentlichen der fixen Kosten, nach dem Tragfähigkeitsprinzip, wird auf die Belastbarkeit der Kostenträger abgestellt, d. h. die Kosten werden nicht verursachungsgerecht, sondern z. B. aufgrund des erzielbar hohen oder niedrigen Marktpreises des Kostenträgers verteilt. Vgl. Hentze, J., Kehres, E. (1995), S. 42, und Vgl. Keun, F. (1999), S. 165.

440 Vgl. Strehlau-Schwoll, H. (1997), S. 542.
} 


\subsubsection{Teilkostenrechnung}

Die Teilkostenrechnungssysteme lehnen die Zurechnung sämtlicher Kosten auf die Leistungseinheit generell ab. Sie unterscheiden sich aber durch den Umfang der Kostenzurechnung untereinander. Sie beschränken sich auf die Zurechnung der variablen Kosten je Kostenträger ${ }^{441}$ und stellen somit entscheidungsorientierte Informationen für die Unternehmenssteuerung bereit. ${ }^{442}$ Die Grundlage ist also hier nicht nur die Trennung von zeitabhängigen und leistungsabhängigen Kostenbestandteilen unter dem Aspekt einer veränderten Beschäftigung, sondern die alleinige Weiterverrechnung der leistungsabhängigen Kosten auf die Kostenträger bzw. auf die Leistungsarten. Die restlichen Kosten werden dabei lediglich im Rahmen einer betrieblichen Ergebnisrechnung in erweiterten Abrechnungskreisen berücksichtigt. $^{443}$

Da die Teilkostenrechnung langfristige Entscheidungen und Kostenkontrolle und damit Aussagen der Vollkostenrechnung nicht ersetzen kann, ${ }^{444}$ und um die Informationen für Planungs-, Steuerung- und Kontrollzwecke zu nutzen, muss das Teilkostenrechnungssystem analog zur Vollkostenrechnung auf Plan- und Istkostenbasis konzipiert sein. ${ }^{445}$ Kritikpunkten, dass die Anwendung eines Parallelkostenrechnungssystems zu mehr Aufwand führt, kann entgegengehalten werden, dass lediglich die Installation einen einmaligen Aufwand darstellt. Die laufende Erfassung der Daten erfolgt dann nur einmal für beide Systeme.

Die Grenzplankostenrechnung stellt eine Weiterentwicklung der flexiblen Plankostenrechnung auf Teilkostenbasis dar, deren Rechnungsziel in der Bereitstellung von Kosteninformationen für kurzfristige Planung und Kontrolle besteht. ${ }^{446}$ Der entscheidende Unterschied zu einer Plankostenrechnung auf Vollkostenbasis ist dabei, dass sowohl bei der Bildung von Verrechnungssätzen für innerbetriebliche

\footnotetext{
${ }^{441}$ Vgl. Gabele, E., Fischer, P. (1992), S. 205.

442 Vgl. Rohrig, R., Schnee, S. (1995): Kostenarten, Kostenstellen, Kostenträgerrechnung, in: Eichhorn, S., Schmidt- Rettig, B. (Hrsg.): Krankenhausmanagement im Werte- und Strukturwandel, Stuttgart, Berlin, Köln, 1995, S. 299.

${ }^{443}$ Vgl. Coenenberg, A. (1992), S. 114.

444 Vgl. Rohrig, R., Schnee, S. (1995), S. 303.

445 Vgl. Schweitzer, M., Küpper, H. U. (1995), S. 371.

446 Kilger, W. hat in Form der Grenzplankostenrechnung ein Instrument für eine fundierte Planung und Kontrolle auf Basis der Teilkostenrechnung geschafften. Vgl. Troßmann, E. (1996), S. 226.
} 
Leistung als auch bei der Ermittlung von Kalkulationssätzen nur die proportionalen Plankosten berücksichtigt werden. ${ }^{447}$

In der Teilkostenrechnungssysteme sind zwei Ausprägungen zu unterscheiden: Teilkostenrechnungssysteme auf Basis variabler Kosten und Teilkostenrechnungssysteme auf Basis von Einzelkosten.

Bei der Teilkostenrechnung auf Basis variabler Kosten werden lediglich die beschäftigungsproportionalen Kostenbestandteile (variablen Kosten) auf die Kostenträger verteilt. Hier können Fixkosten entweder als Block bei der Durchführung der Betriebsergebnisrechnung in einer Summe (einstufiges Direct Costing) ausgewiesen werden, womit der Vergleich zwischen den Erlösen und den variablen Kosten einen Deckungsbeitrag ermöglicht wird, der zur Steuerung des Erfolges geeignet erscheint. Die Fixkosten können auch in mehrere Blöcken aufgeteilt werden (mehrstufige Deckungsbeitragsrechnung bzw. stufenweise Fixkostendeckungsrechnung). Hiermit kann eine Analyse und eine Darstellung des Fixkostenblocks sowohl bezüglich der zeitlichen Dimension als auch bezüglich der Zuordnung zu bestimmten Kostenträgergruppen ermöglicht werden. ${ }^{448}$

Bei der Teilkostenrechnung auf Basis von Einzelkosten werden nur die Einzelkosten den Kostenträger zugerechnet. Die relative Einzelkostenrechnung (Einzelkosten- und Deckungsbeitragsrechnung) löst sich von der Unterscheidung in variable und fixe Kosten. Durch Bilden von Bezugsgrößenhierarchien wird es möglich, alle Kosten an einer Stelle als Einzelkosten zu verrechnen. Einzelkosten entstehen in bestimmten Hierarchiestufen und sie können nicht nur bezogen auf den Kostenträger als Bezugsgröße, sondern auch im Hinblick auf andere Bezugsgrößen analysiert werden.

\subsubsection{Ausgewählte Kostenverfahren in Krankenhaus}

Die Auswahl über ein Kostenverfahren besteht nicht in einer Entscheidung für oder gegen die Voll- oder Teilkostenrechnung (noch weniger in einer Entscheidung

\footnotetext{
447 Vgl. Troßmann, E. (1996), S. 194.

${ }^{448}$ Vgl. Strehlau-Schwoll, H. (1993), S. 214.
} 
zwischen Zuschlagskalkulation oder Deckungsbeitragsrechnung), sondern in der Entscheidung für die Abrechnung der jeweils relevanten Kosten, d. h. in der Entscheidung für eine Relevanzkostenrechnung. Die Kostenrechnungstheorie hat mehrere Kostenrechnungsverfahren entwickelt, wobei für die unterschiedlichen Abrechnungszwecke ein geeignetes Instrument zur Verfügen stellen, aber nicht unbedingt ein Verfahren für alle Abrechnungszwecke darstellen. ${ }^{449}$ Bei der Entscheidung für ein bestimmtes Kostenrechnungsverfahren kommt es daher auf die spezielle Eignung dieses Verfahrenes für die Erreichung des jeweiligen Abrechnungszieles an. ${ }^{450}$

In der Praxis der Krankenhäuser ist für Zwecke der Preisbildung sowie zur Erstellen des Kosten- und Leistungsnachweises eine Plankostenrechnung auf Vollkostenbasis unverzichtbar.

Für Zwecke der Wirtschaftlichkeitsmessung und zur Steuerung des betrieblichen Geschehens müssen jedoch die Istkosten den Normalkosten gegenübergestellt werden, wobei der Normal-Istvergleich sämtlicher Leistungen einschließlich der Hilfs- und Nebenleistungen erfassen muss. Der Normal-Istvergleich von Deckungsbeiträgen reicht zur Wirtschaftlichkeitsmessung nicht aus, denn ein höherer Deckungsbeitrag kann sowohl auf höhere Erträge als auch auf geringere direkte Kosten zurückzuführen sein. Die Wirtschaftlichkeitsmessung im bedarfsdeckungswirtschaftlich ausgerichteten Krankenhaus erstreckt sich auf die sogenannte „Kostenwirtschaftlichkeit“, d. h. auf die Feststellung des sparsamen Mitteleinsatzes, nicht auf die Messung der „Erfolgswirtschaftlichkeit“ einzelner Leistungsarten, da die Krankenhäuser keine Sortimentspolitik betreiben können, sondern die zur Deckung des Bedarfs erforderlichen Leistungen ohne Rücksicht auf den finanziellen Leistungserfolg anbieten müssen. Da in den meistens Krankenhäusern alle Kosten über die Kostenstellenrechnung geleitet worden sind, können die Kosten auf Kostenträger unter Umgehung der Kostenstellenrechnung direkt zugeordnet werden. Die Teilkostenrechnung kann aber auch ohne eine Verrechnung von Kosten innerhalb des Kostenstellensystems durchgeführt werden.

\footnotetext{
449 Davis Weintraub, L., Dube, R. J. (1994), S. 114-115.

${ }^{450}$ Vgl. Hübner, H. (1980), S. 161.
} 
Für eine Teilkostenrechnung im Krankenhaus wird das Rechnen mit relativen Einzelkosten empfohlen, da dies Konzept die Kostenverantwortlichkeit in Vordergrund stellt und für die betriebliche Steuerung und Wirtschaftlichkeitskontrolle im Krankenhaus besonders geeignet ist. $^{451}$. Das bedeutet, Kostenträgerrechnung, Wirtschaftlichkeitskontrolle und Steuerung des betrieblichen Geschehens erfolgen bei einer Teilkostenrechnung auf Basis der Kostenstelleneinzelkosten. Beispielsweise stellen die Personalkosten des ZentralOP bezogen auf die einzelne Operation Gemeinkosten dar, da diese Kosten von der Entscheidung einer einzelnen Operation nicht abhängig sind. Andererseits sind diese Kosten Einzelkosten der Kostenstellen OP-Abteilung, da sie abhängig von der Entscheidung, einer OP-Abteilung zu betreiben, sind.

Wesentliche und besondere Merkmale der relativen Einzelkostenrechnung sind die von anderen Kostenrechnungssystemen abweichende Betrachtung von relativen Einzelkosten sowie die Vorgabe, Kosten im Form einer Grundrechnung ohne Zielorientierung der Auswertung zu erfassen. So wird im Sinne der relativen Einzelkosten zwischen Leistungskosten und Bereitschaftskosten unterschieden, wobei die Bereitschaftskosten (wie Personalkosten im oben genannten Beispiel) in ihrer zeitlichen Dimension (monatlich, Quartalsjahr oder Halbjahr) bis hin zu mehrperiodische Kosten aufgeschlüsselt werden. Dadurch ist für den Kostenverantwortlichen ein Instrument entstanden, dass auch geeignet ist, strategische Entscheidungen zu unterstützen. ${ }^{452}$

\subsection{3: Prozesskostenrechnung als modernes Kostenrechnungsverfahren}

Als modernes Kostenrechnungssystem wird in erster Linie die Prozesskostenrechnung als Ergänzung zu den traditionellen Kostenrechnungssystemen für Dienstleistungsunternehmen diskutiert. ${ }^{453}$

\footnotetext{
451 Tuschen, K. H. (1988), S. 14

452 Für die konkrete Aufgabenstellung der Kostenrechnung im Krankenhaus ist diese Differenzierung der Kosten in Leistungs- und Bereitschaftskosten so interessant, weil die Bereitschaftskosten einen hohen Anteil der Kosten im Krankenhaus sind. Vgl Strehlau-Schwoll, H. (1993), S. 214215.

453 Horvath, P., Mayer, R. (1995): Konzeption und Entwicklung der Prozesskostenrechnung. In: Mannel, W. (Hrsg.), Prozesskostenrechnung. Wiesbaden, 1995, S. 59-61.
} 
Aufgrund der Zunahme des Anteils der (fixen) Gemeinkosten an den Gesamtkosten eines Produktes bzw. einer Dienstleistung, ${ }^{454}$ der mit den traditionellen Verfahren der Kostenrechnung, die den Fokus ihrer Betrachtung hauptsächlich auf die Verrechnung der Einzel- bzw. der direkten Gemeinkosten richten, zu global verrechnet werden und nicht mehr verursachungsgerecht den betreffenden Kostenträger zugeordnet werden konnte, wurde Mitte der achtziger Jahre die Prozesskostenrechnung entwickelt. ${ }^{455}$

Die Prozesskostenrechnung auch „Activity-Based-Costing“ System, oder „Cost Driver Accounting“ ist ein Instrument, das zur Planung und Steuerung der Kosten in den indirekten Bereichen des Unternehmens bzw. zu deren Verrechnung auf Produkte dient. ${ }^{456}$ Als grundsätzliches Merkmal muss herausgestellt werden, dass die Prozesskostenrechnung ihrem Wesen nach in der Regel eine Vollkostenrechnung darstellt. ${ }^{457}$ Das bedeutet, die Prozesskostenrechnung bedient sich der traditionellen Kostenarten- und Kostenstellenrechnung. ${ }^{458}$ Insofern handelt es sich um kein völlig neues Kostenrechnungssystem. Davon unabhängig ist bei der Einführung der Prozesskostenrechnung eine Analyse und gegebenenfalls eine Umstrukturierung von Kostenarten- und Kostenstellen angebracht.

Die Ziele der Prozesskostenrechnung sind zum einen die möglichst verursachungsgerechte Zuordnung der beschäftigungstunabhängigen Kosten auf die Produkte. Die Prozesskostenrechnung ist dem Verursachungsprinzip in besonderem

\footnotetext{
${ }^{454}$ In hochtechnologisierten Betriebe werden Ausdrücklich die Tätigkeiten, die immer stärker geplant, gesteuert, überwacht und kontrolliert werden müssen, in den sogenannten indirekten Leistungsbereichen dominiert. Diese Aktivitätsverschiebung hat erhebliche Auswirkung auf die betriebliche Kostenstruktur zur Folge. Das bedeutet im Einzelnen, dass die ansteigende Automatisierung und Flexibilisierung in dem Betrieb zu einem Anstieg des Anteils der Einzelkosten an den Gesamtkosten führen. Vgl. Keun, F. (1997) S. 193.

455 Den Anstoß für die Entwicklung der Prozesskostenrechnung gaben 1985 Miller und Vollmann in ihrem Artikel „The hidden factory“, in dem sie darauf hinwiesen, dass den indirekten Unternehmensbereichen besondere Bedeutung zukommt, da deren Kosten ständig ansteigen und damit die Wettbewerbsfähigkeit und Rentabilität der Unternehmen beeinträchtigen. Bei der Analyse der indirekten Unternehmensbereiche stellen sie einen Zusammenhang zwischen der Höhe der Gemeinkosten und der Ausführung von Transaktionen in den indirekten Bereichen fest. Vgl. Götze, U., Meyerhoff, J. C. (1993): die Prozeßkostenrechnung - Stand und Entwicklungstendenzen, in: Zeitschrift für Planung, 1/ 1993, S. 67.

${ }^{456}$ Vgl. Götze, U., Meyerhoff, J. C. (1993), S. 66.

457 Vgl. Horvath, P., Mayer, R. (1989): Prozesskostenrechnung, Der neue Weg zu mehr Kostentransparenz und wirkungsvolleren Unternehmensstrategien, In: Controlling, 7/1989, S. 216.

${ }^{458}$ Vgl. Götze, U., Meyerhoff, J. C., (1993), S. 67
} 
Maße verpflichtet. Sie ist mit dem Anspruch entwickelt worden, eine verursachungsgerechteres Kostenrechnungssystem zu sein. Die Kosten sollen dem Verursachungsprinzip zufolge von der Kostenstelle, dem Kostenträger und der Periode getragen werden, durch die bzw. den sie verursacht werden. ${ }^{459}$ Zum anderen hat sie das Ziel der effizienten Steuerung der Gemeinkosten. Ziel der Prozesskostenrechnung ist es, eine höhere Transparenz in den Gemeinkostenbereichen $\mathrm{zu}$ ermöglichen und in Verbindung mit einem effizienten Prozessmanagement eine gezielte Steuerung des Ressourcenverbrauchs zu bewirken. $^{460}$

Die Anwendung der Prozesskostenrechnung im Krankenhaus ist prinzipiell relevant, weil sich die Kostenstrukturen im Krankenhaus durch einen hohen Anteil an fixen Gemeinkosten auszeichnen. ${ }^{461}$

Bei den Krankenhäusern rechnet die Prozesskostenrechnung die Gemeinkosten nach Maßgabe kostenstellenübergreifender Aktivitäten zu, da die Erzeugung der Krankenhausleistung einer Vielzahl von Prozessen innerhalb und außerhalb des Krankenhauses bedarf, die sich aus primären und sekundären Teilprozessen zusammensetzen. Der gesamte Behandlungsprozess kann durch die stark tätigkeitsorientierte Leistungserstellung anhand von Teilprozessen moduliert werden. ${ }^{462}$ Die Teilprozesse im Krankenhaus werden in Abb. 33 dargestellt.

\footnotetext{
${ }^{459}$ Vgl. Coenenberg, A. (1993), S. 48.

${ }^{460}$ Vgl. Götze, U., Meyerhoff, J. C., (1993), S. 69.

461 Vgl. Chan Yee-Ching, L. (1994): Improving Hospital Cost Accounting with Activity-Based Costing, in: Finkler, S. A. (Edit.),Issues in Cost Accounting for Health Care Organisations, New York, 1994, S. 267.

${ }^{462}$ Vgl. Graf, V. (1999): Prozessoptimierung im Krankenhaus, steigert die Effizienz und festigt die Kundenbindung, in: F\&W, 6/ 1999, S. 520.
} 


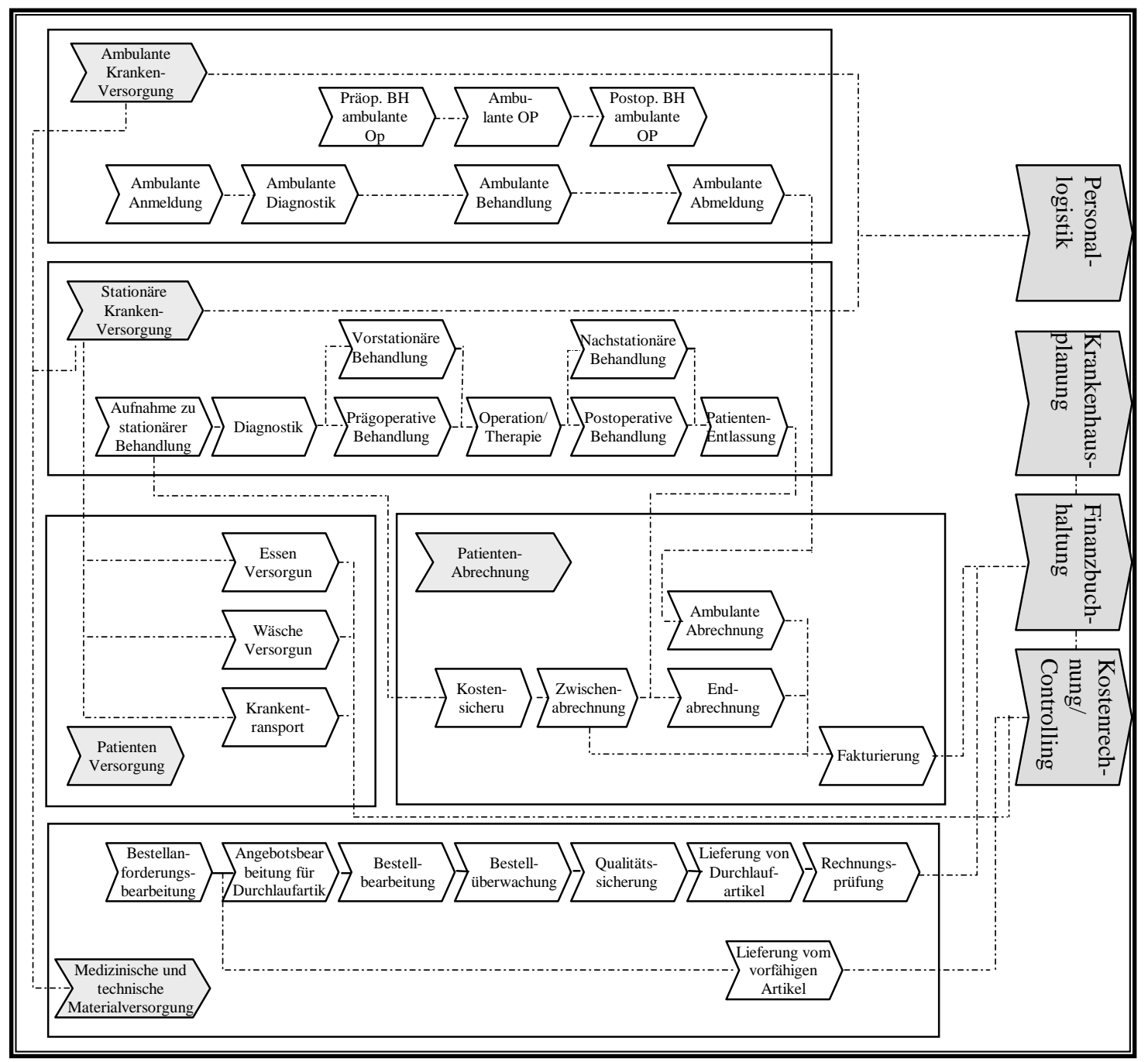

Abb. 33: Teilprozesse im Krankenhaus. ${ }^{463}$

Entscheidend für die Aussagekraft der Prozesskostenrechnung im Krankenhaus ist die Entwicklung einer angemessenen Prozessstruktur. Dies bedeutet im Einzelnen:

- Konzentration auf die Krankenhausbereiche, die einen hohen Anteil an Gemeinkosten haben und nicht bereits durch andere Kostenrechnungsinstrumente detailliert verfolgt werden.

- Sukzessive Einführung (z. B. erst der Materialwirtschaft im Krankenhaus, dann einzelne weitere Abteilungen).

- Einsatz als Managementinstrument zunächst parallel zur traditionellen Kostenrechnung. ${ }^{464}$

463 Quelle: Scheer, A. W. (1996): Prozessmanagement im Krankenhaus, in: Adam, D. (Hrsg.) Krankenhausmanagement, Schriftenreihe zur Unternehmensführung, Band 59, Wiesbaden, 1996, S. 79. 
Die wichtigsten grundlegenden Schritte für die praktische Einführung des Prozesskostenrechnungssystems sind die folgenden: ${ }^{465}$

1 Tätigkeitsanalyse zur Bestimmung von Teilprozessen in den Kostenstellen: Es wird für jede Abteilung separat ermittelt, welche Tätigkeiten innerhalb des Untersuchungszeitraums durchgeführt werden und wie groß der benötigte Bedarf in Prozent der Gesamtkapazität ist. Beispielsweise können in der Abteilung „Entbindung durchführen“ die Personalkosten in 50\% normale Entbindungen, 10\% Kaiserschnittentbindung und 40\% sonstige gynäkologische Leistungen geteilt werden. Jeder Teilprozess wird auf durchführende Kostenstellen und Hauptprozesse zugeordnet. Dies bedeutet eine kostenstellenbezogene Information über die Art und den Umfang sämtlicher physischer und wertmäßiger Teilprozesse (z. B. der Teilprozess „Kontrolle der Herztöne des Kindes“ als Bestandteil des Hauptprozesses „Entbindung durchführen“).

2 Ermittlung von Bezugsgrößen und Planprozessmengen: Neben der Ermittlung der Teilprozesse wird in der Tätigkeitsanalyse festgelegt, welche kostentreibenden Faktoren (sogenannte „Cost Drivers“) als Bezugsgrößen für die volumenabhängigen leistungsmengeninduzierten Prozesse geeignet sind. Von ihnen wird angenommen, dass der Ressourcenverbrauch im Krankenhaus direkt oder indirekt von der Anzahl der erbrachten Leistungseinheiten abhängt. Die Bezugsgrößen sind so auszuwählen, dass die dazugehörige Prozessmenge schnell, wirtschaftlich und genau erfasst werden kann. ${ }^{466}$ Prozesse und Teilprozesse, die keinen Bezug zum Produktions- bzw. Leistungsvolumen haben, werden als volumenunkabhängige bzw. Leistungsmengenneutrale Prozesse bezeichnet. In Tab. 21 werden beispielsweise die Bezugsgrößen für Teilprozesses stationäre „Krankenversorgung“ dargestellt.

\begin{tabular}{||l|l|l||}
\hline Bereich & Teilprozess & Bezugsgrößen \\
\hline Pflege & $\begin{array}{l}\text { Patienten auf der Station } \\
\text { aufnehmen }\end{array}$ & $\begin{array}{l}\text { Anzahl der aufzunehmenden } \\
\text { Patienten }\end{array}$ \\
\hline
\end{tabular}

464 Vgl. Kolb, T. (1994): Prozesskostenrechnung im Krankenhaus, in: F\&W, 5/ 1994, S. 396.

465 Vgl. Götze, U., Meyerhoff, J. C. (1993), S. 69-72, Keun, F. (1997) S.194-197 und Kolb, T. (1994) S. 396-397.

${ }^{466}$ Vgl. Freidank, C, (1994). S. 340. 


\begin{tabular}{||l|l|l||}
\hline \multirow{3}{*}{$\begin{array}{l}\text { Ärztlicher- } \\
\text { Bereich }\end{array}$} & Patienten waschen & $\begin{array}{l}\text { Anzahl der zu waschenden } \\
\text { Patienten }\end{array}$ \\
\cline { 2 - 3 } & Bett herrichten & Anzahl der stationären Patienten \\
\cline { 2 - 3 } & Mittagessen austeilen & Anzahl der stationären Patienten \\
\hline & Anamnesen durchführen & $\begin{array}{l}\text { Anzahl der aufzunehmenden } \\
\text { Patienten }\end{array}$ \\
\cline { 2 - 3 } & $\begin{array}{l}\text { Infusion Verabreichen } \\
\text { Patienten bei einem }\end{array}$ & Anzahl der Infusionen \\
\cline { 2 - 3 } & $\begin{array}{l}\text { Abschlussuntersuchung der stationären Patienten } \\
\text { durchführen }\end{array}$ & Anzahl der entlassenen Patienten \\
\hline
\end{tabular}

Tab. 21: Bezugsgrößen für Teilprozesses „stationäre Krankenversorgung““ ${ }^{467}$

3 Ermittlung der Plankosten je Prozess und Bildung von Prozesskostensätze: Im dritten Schritt werden die Plankosten je Prozess und die gesamten Kosten der Planprozessmenge ermittelt. Durch Division der gesamten auf eine Planprozessmenge entfallenden Kosten durch die einzelnen Teilaktivitäten können die Kosten der Teilprozesse ermittelt werden. Die Zurechnung der Gemeinkosten auf die Trägereinheiten erfolgt mit Hilfe der Prozesskostensätze und der produkt-/leistungsbezogenen in Anspruch genommenen Aktivitäten der jeweiligen indirekten Leistungsbereiche. Grundlage für die Zuordnung kann eine Kostenartenweise analytische Planung sein. Kosten der leistungsmengenneutralen Prozesse (z. B. die Personalkosten der Stationsleitung, deren Tätigkeit keine Korrelation zwischen Prozess und Kostentreiber erkennen lässt) können mit Hilfe von Zuschlagsätzen den Leistungseinheiten zugeordnet werden.

Das Ziel der Prozesskostenrechnung im Krankenhaus ist wie bei anderen Unternehmen die möglichst verursachungsgerechte Zuordnung der Gemeinkosten. Durch die Anwendung der Prozesskostenrechnung können Kostenstrukturen und Abläufe transparent dargestellt. Die Prozesskostenrechnung zwingt zur Erfassung und Strukturierung der einzelnen Tätigkeiten, Vorgänge und Aktivitäten sowie

${ }^{467}$ In Anlehnung an: Schmidt-Rettig, B., Böhning, F. (1999): Bedeutung und Konzeption einer Prozesskostenrechnung im Krankenhaus, in: Eichhorn, S., Schmidt-Rettig, B. (Hrsg.), Profitcenter und Prozessorientierung, Optimierung von Budget, Arbeitsprozesses und Qualität, Stuttgart, Berlin, Köln, 1999, S. 121. 
deren Quantifizierung im Krankenhaus. Dies führt zu einer Identifizierung der Kostentreiber. Sie stellen die Einflußgrößen auf fixe Kostenanteile im Krankenhaus dar und sind Maßstäbe der Effektivität und Effizienz des Bemühens um die krankenhausbetriebliche Leistungserstellung. ${ }^{468}$

Durch die Anwendung der Prozesskostenrechnung, wobei ein hohes Niveau von Kostenbewußtsein, Analysefähigkeit, Kommunikation und Datenfluss benötigt wird, wird auch ein entscheidender Beitrag zur Planung, Steuerung und Kontrolle der Gemeinkosten geliefert. ${ }^{469}$ Einerseits verbindet sie das Kostenstellenbudget mit dem dahinterstehenden Mengengerüst. Über eine leistungs- und mengenorientierte Gemeinkostenplanung sowie laufende Soll-Ist-Vergleiche läßt sich die Qualität von Kostenplanung und Kostensteuerung erheblich verbessern. Mit Hilfe der Kostentreiber sind Fixkosten auf ihre Angemessenheit zu überprüfen. Anderseits ergibt sich durch die genauere Abbildung der Kostenbeziehungen und Kostenabhängigkeiten der Verrechnung der innerbetrieblichen Sekundärleistungen eine bessere Leistungskalkulation. Darüber hinaus kann mit Hilfe der Prozesskostenrechnung eine strategische Kalkulation als Grundlage strategischer Entscheidungen durchgeführt werden. ${ }^{470}$

Generell ist die Anwendung der Prozesskostenrechnung im Krankenhaus besonders für langfristiges Kostenmanagement geeignet, da sie Rationalisierungspotentiale durch feine Optimierung der Arbeitsläufen ermöglicht und gleichzeitig mehr Kunden- und Qualitätsbewußtsein entstehen läßt.

\subsection{Systeme der Kosten- und Leistungsrechnung in deutschen Kranken- häusern}

Infolge gesetzlicher, regionaler, trägerschaftlicher und organisatorischer Festlegungen ist der Spielraum für kurzfristige Alternativentscheidungen, z. B. beim Leistungsprogramm, im Krankenhaus in Deutschland eingeschränkt. Der

\footnotetext{
${ }^{468}$ Kolb, T., (1994) S.397.

${ }^{469}$ Vgl. Horvath, P. (1996), S.536.
} 
größte Teil der Absatzpreise, vor allem die Pflegesätze, sind durch die BPflV als Vollkostenpreis festgelegt.

Nach den vorangegangen Überlegungen wird deutlich, dass der nur Benutzerkosten enthaltenden Pflegesatz nicht etwa eine Teilkostenrechnung bedeutet. Abgrenzungskriterium ist hier nicht die verursachungsgerechte Zurechenbarkeit, sondern die duale Finanzierung. Dabei wird davon ausgegangen, dass in deutschen Krankenhäuser zunächst die traditionelle Vollkostenrechnung als Istkostenrechnung eingeführt wird. ${ }^{471}$ Die nächste Entwicklungsstufe könnte die Vollplankostenrechnung sein, die zwar bereits eine Kostenauflösung erfordert, aber nur mit Wirkung für die Kostenstellen. Schließlich wird bei Änderung der Gesetzlage die Weiterentwicklung zur Teilkostenrechnung zweckmäßig sein.

Obwohl der deutsche Gesetzgeber in $\S 8$ KHBV Abs. 3 und Abs. 4 die Zurechnung der Vollkosten auf Kostenstellen verlangt, kann man trotzdem nicht behaupten, dass die Vollkostenrechnung vorgeschrieben wird, da die Zurechnung sämtlicher Kosten auf Kostenstellen und Kostenträger auch in der Teilkostenrechnung möglich ist.

Andererseits verliert der Methodenstreit in diesem Punkt an Bedeutung, da Vollund Teilkostenrechnung im Krankenhaus denselben Ausgangspunkt haben, nämlich die gesamten Kosten des Krankenhauses in Form von Kostenstellenkosten. Es ist unproblematisch, für Zweck der betrieblichen Steuerung und Wirtschaftlichkeitskontrolle die Kostenrechnung als Teilkostenrechnung zu nutzen und zusätzlich Vollkosten für Zwecke der Selbstkostenermittlung zu berechnen. ${ }^{472}$

Die Prozesskostenrechnung als Gestaltungsgrundlage der Kostenrechnung in deutschen Krankenhäusern kommt vermehrt in Frage. Die Anwendung der Prozesskostenrechnung ist besonders interessant in den indirekten Bereichen (z. B. Verwaltung, Materialbeschaffung), die nach den gesetzlichen

${ }^{470}$ Vgl. King, M., Lapsly, I, Mitcbell, F., Moyes, J. (1994): Activity Based Costing in Hospital, A Case Study Investigation, The Chartered Institute of Management Accountants, London 1994, S. 11.

${ }^{471}$ Vgl. Bopp, M., Hohenbild, R. (1984) S. 59.

472 Vgl. Hübner, H. (1980) S. 119 
Mindestanforderungen ${ }^{473}$ durch Sonderentgelt oder Fallpauschalen, aber noch ohne verursachungsgerechte Kostenzuordnung pauschal über den Kostenträger „Basispflegesatz“ abgerechnet bzw. vergütet werden. ${ }^{474}$

Darüber hinaus ist die Konzeption der Prozesskostenrechnung mit der Einführung einer patientenorientierten Kostenträgerrechnung nicht mehr neu, da das Verfahren der Verrechnungssatzkalkulation stark der Prozesskostenrechnung gleicht. Bei der Prozesskostenrechnung steht die Definition von Prozesses und nicht von Leistungen im Mittelpunkt. Indem ein Großteil der Prozesse im Krankenhaus am Patienten erfolgt, überschneiden sich jedoch Leistungs- und Prozessdefinition sowie die Wahl der Bezugsgrößen in einer Vielzahl der Fälle miteinander. Mit der Einführung von Sonderentgelte und Fallpauschalen, die als fallorientierte Entgelte geradezu eine prozessorientierte Betrachtung dieser Leistungen erzwingen, ist die Einführung der Prozesskostenrechnung im Krankenhaus leichter geworden. Aus denen sind vielen Schritten zu gewonnen wie Tätigkeitsanalyse zur Bildung und Beschreibung der Prozesse, Bestimmung der Kostenstellen und Kostentreiber, Bestimmung der Bezugsgrößenmengen bzw. Kostenvolumen und Plankosten für die Kostenstellen und Ermittlung der Prozesskostensätze.

Praktisch hat die Anwendung der Prozesskostenrechnung jedoch einige Nachteile. Mit diesem System können nicht alle Gemeinkosten verteilt werden z. B. ein Rest an leistungsmengenneutralen Prozessen. Weiterhin ist sie komplizierter als klassische Rechnungsverfahren, z. B. bedeutet Kostenstellen mit mehr als einem Kostentreiber eine höhere Komplexität der Verrechnung. Und schließlich fordert sie einen höheren Informationsbedarf, z. B. im Sinne der Mehrfacherfassung von Informationen in verschiedenen isolierten Informationssystemen sowie einen hohen Kommunikations- und Koordinationsaufwand, der sich auf Wirtschaftlichkeit und

\footnotetext{
473 Der Umsatz des neuen Entgeltsystems nach BPflV macht es erforderlich, sämtliche Kosten der einzelnen Leistung möglichst verursachungsgerecht zuzuordnen. Während dies bei Einzelkosten ohne Probleme möglich ist, scheitert beispielsweise die klassische Kostenträgerrechnung an einer sehr pauschalen Zurechnung der Gemeinkosten über Zuschlagssätze. Im Gegensatz zu der objektbezogenen Kostenrechnung dieses Kostenrechnungssystems orientiert sich die Vorgehensweise der Prozesskostenrechnung zeitraum- und ablauforientiert. Nach Maßgabe eines Prozesses, der zeitlich und organisatorisch klar definiert werden kann, werden Gemeinkosten für einen Vorgang, den Fall, ermittelt, dessen Kostenerstattung durch Sonderentgelt oder Fallpauschale pauschal vergütet wird. Vgl. Kolb, T., (1994) S. 396.

474 Vgl. Trill, R. (1996): Krankenhaus-Management, Aktionsfelder und Erfolgspotentiale, Neuwied, 1996, S. 173.
} 
Leistungsfähigkeit der Patientenbehandlung auswirkt. ${ }^{475}$ Hierbei ist ein ausreichender Schulungs- und Informationsaufwand zu betreiben. Damit ist eine Prozesskostenrechnung in deutschen Krankenhäuser nicht „auf einen Schlag“ einzuführen. Der Implementierungsprozess muss behutsam, gegebenenfalls durch parallele Erfassung der Kosten in dem bisherigen Kostenrechnungssystem erfolgt. ${ }^{476}$ Aus den vorangegangenen Überlegungen lässt sich entnehmen, dass die Anwendung der Prozesskostenrechnung im deutschen Krankenhäuser im Bereich der operativen Leistungen jedoch schon heute der Fall ist.

\subsection{Systeme der Kosten- und Leistungsrechnung im syrischen Krankenhaus}

Der syrische Gesetzgeber hat kein bestimmtes System für Kosten- und Leistungsrechnung benannt. Allerdings ist es mit Betrachtung der detaillierten Anforderung von Erfassung und Verrechnung der Kosten zu sehen, dass generell nach dem SERS die Kosten auf Teilkostenrechnungsbasis zu verrechnet werden müssen. Allerdings hat der syrische Gesetzgeber es erlaubt, jeder Wirtschaftsbereich individuell ein System auswählt, das dem Betrieb geeignet und verwendbar ist. Das ausgewählte System kann den gesonderten Aktivitäten des Wirtschaftsbereichs geeignet sein, darf aber die gesetzlichen Vorschriften über Kostenerfassung, -ermittlung und -verrechnung nicht umgehen. ${ }^{477}$

Darüber hinaus muss nach dem SERS die Kosten- und Leistungsrechnung gemäß des Systems der Teilkostenrechnung auf Istkosten- sowie Normalkostenbasis durchgeführt wird. Die Normalkostenrechnung wird als Instrument für die Kontrolle der Kosten und für die Erhöhung der Wirtschaftlichkeit vom SERS genannt. $^{478}$

Im Einzelnen sind Direktkosten, Indirektkosten und Gemeinkosten zu unterscheiden. Das SERS hat als Vorschriften für Verrechnung der Kosten gefördert, dass die Direktkosten separat in einer Verrechnungsstufe ermittelt werden sollen. Dann sollen die Indirektkosten weiter den Kosten zugerechnet

\footnotetext{
${ }^{475}$ Vgl. Schlüchtermann, J., Gorschlüter, P. (1996), S. 108.

476 Vgl. Kolb, T., (1994), S. 401.

477 Vgl. § 81 SERS.

${ }^{478} \mathrm{Vgl}$ § 16 SERS.
} 
werden. Dagegen dürfen die Gemeinkosten den Kosten nicht zurechnet werden, sondern sollen in der Erfolgsrechnung von den Erlösen abgezogen werden.

Direktkosten sind diejenige, die an einer „Produktionskostenstelle“ (Kostenstelle der Behandlungs- und Pflegeprozess in Pflegefachbereichen) direkt verrechnet werden können. Sie sollen an der beziehenden Kostenstelle separat ermittelt werden. Direktkosten erfassen die direkten Personalkosten, direkten Materialkosten und kostenstellenbezogenen Abschreibungen. Durch Dividieren der ermittelten Direktkosten durch die Leistungseinheiten resultieren die so genannten Direktkosten der Leistungseinheit. ${ }^{479}$

Indirektkosten sind diejenige Kosten, die leistungsbezogen sind, aber an einer „Produktionskostenstelle“ (Kostenstelle der Behandlungs- und Pflegeprozess in Pflegefachbereiche) nicht direkt, sondern indirekt über die Produkthilfskostenstellen (Medizinische-Institutionen-Kostenstellen und Versorgungs-Kostenstellen) verrechnet werden können. Alle diese Indirektkosten sollen in einer zweiten Rechnungsstufe auf die leistungsempfangenden Kostenstellen verteilt werden. Die Verteilung der Kosten muss möglichst auf Grundlage tatsächlich beanspruchter Leistungen (innerbetriebliche Leistungsverrechnung) oder auf Grundlage von Verteilungsschlüsseln (Umlagenrechnung) durchgeführt werden.

Gemeinkosten sind die verbleibenden Kosten, die an allgemeinen Kostenstellen (administrative Kostenstellen und wirtschaftliche Kostenstellen) berechnet sind. Diese Kosten sollen als ein Block in der Ergebnisrechnung behandelt werden.

\section{DISKUSSION UND ERGEBNISSE}

\subsection{Allgemeine Betrachtungen}

Aufgrund seiner Funktion, seiner exponierten Position und den seitens der Patienten, der Öffentlichkeit und der Politik formulierten Erwartungen wird das Krankenhaus nicht umhin kommen, unternehmerische Aspekte in seine Betriebstätigkeiten zu integrieren. Und wenn man den Stellenwert des Anteils des Gesundheitswesens an der gesamten Wirtschaft eines Landes betrachtet, wunderte es, dass 
sich diese Betrachtungsweise nicht schon früher angebahnt hat. In der letzten Jahren wurde das Krankenhaus jedoch langsam, aber sicher als Unternehmen angesehen. ${ }^{480}$

Das Krankenhaus als Unternehmen, das nach Wirtschaftlichkeits-, Markt- und Wettbewerbsprinzipien arbeiten soll, hat unbedingt die betriebswirtschaftlichen Verfahren des modernen Managements zu anwenden. Vor allem ist eine strategische sowie operative Planung zur Absicherung der Zukunft eines Krankenhauses lebensnotwendig geworden. ${ }^{481}$

Auf Grund verschiedenen Besonderheiten der Leistungserstellung im Krankenhaus, ist der Entwicklungsstand des Krankenhausmanagements nicht vergleichbar mit anderen Industrie- und Dienstleistungsbereichen. Mit dem Hinweis auf die besondere Art der Leistungserstellung, die darüber hinaus auch ethischen moralischen und zunehmend juristischen Fragen unterworfen ist, sind Ansätze einer standardisierten Produktionsplanung unterblieben. ${ }^{482}$

Dieser Entwicklungsstand des Krankenhausmanagements ist von einem Krankenhaus zum anderen unterschiedlich. Dabei sind in vielen Industrienationen die Entwicklungen des Managements relativ weit, z. B. ist das Management der Krankenhäuser in Deutschland schon ab 1972 konzipiert worden, vor allem nach gesetzlichen Orientierungen. Hingegen ist die Situationen anders bei vielen Ländern der Dritten Welt, z. B. sind bisher die Krankenhäuser in Syrien nur selten mit betriebswirtschaftlichen Maßnahmen verwaltet werden. Die Unterschiede können aber auch in einem einzelnen Land auftreten. Beispielsweise haben Privat-Krankenhäuser im Vergleich mit öffentlichen Krankenhäusern ihr Management schon früher und weiter nach dem individuellen Bedarf entwickelt.

Das Entwicklungsniveau eines Landes (die Wirtschaftlage, die Betriebe sowie die Betriebswirtschaftslehre) reflektiert sich auch an dem Entwicklungsstand seiner Krankenhäuser. Der sehr große Unterschied zwischen den Krankenhausbetrieben in Deutschland und Syrien kann auf Unterschiede zwischen den Entwicklungsniveaus

\footnotetext{
${ }^{479} \mathrm{Vgl}$. 4.3.3.

${ }^{480}$ Vgl. Schmitt, R.: Das Krankenhaus auf dem Weg zum Unternehmen, in: das Krankenhaus, 2/ 1987, S. 69 und 72.

${ }^{481}$ Vgl. Abschnitt 2.1.4.
} 
der beiden Ländern zurückgeführt werden. In den folgenden Diagrammen werden allgemeine Kennzahlen über die Wirtschaft, Krankenhäuser sowie Kosten der Krankenhäuser in Deutschland und Syrien zum Vergleich dargestellt.

Das Brutto-Sozial-Produkt betrug 1997 in Deutschland 3658,6 Mrd. DM und in Syrien 31,9 Mrd. DM (0,87 \% in Syrien im Vergleich zu Deutschland), bei 81,6 Mio. Einwohnern in Deutschland und 14,5 Mio. in Syrien. In Deutschland beträgt das Brutto-Sozial-Produkt pro Einwohner 44835,7 DM und in Syrien 2200 DM (4,9\% in Syrien im Vergleich mit Deutschland). ${ }^{483}$ (Vgl. Abb. 34.)

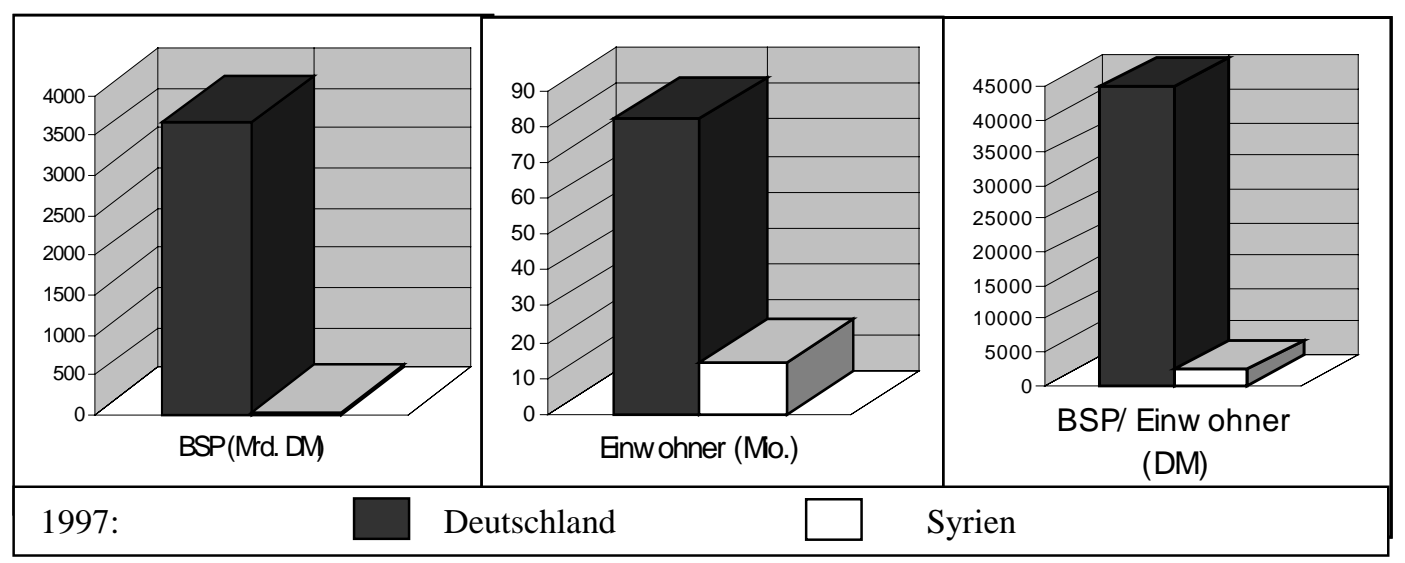

Abb. 34: Brutto-Sozial-Produkt in Deutschland und Syrien.

1998 gab es in Deutschland 2.263 Krankenhäuser und in Syrien 321 Krankenhäuser $(15,5 \%)$. Die Gesamter Bettenzahl betrug 1997 in Deutschland 572.000 Betten und in Syrien 18.737 Betten (3,3 \%). Damit steht für je 10.000 Einwohner 76,7 Betten in Deutschland und 12,9 Betten in Syrien $(16,6 \%)$ zur Verfügung ${ }^{484}(\mathrm{Vgl}$. Abb. 35).

\footnotetext{
482 Vgl. Maltry, H., Strehlau-Schwoll, H. (1997), S. 553.

483 Quelle: Statistisches Bundesamt (1999 b): Mitteilung für die Presse, Erste Ergebnisse nach dem neuen Europäischen System Volkswirtschaftlicher Gesamtrechnungen (ESVG) 1995 für die Jahre 1991 bis 1998, http://www.statistik-bund.de/presse/deutsch/pm/p9152121.htm, 01. 03. 2000, (Stand am: 28. 04. 1999), und Syrische zentrale Statistikamt (1998): Jahresbericht 1997, Damaskus, 1998, S. 96.

484 Quelle: Statistisches Bundesamt (1999 a), S. 1, und Syrische zentrale Statistikamt (1999): Jahresbericht 1998, Damaskus, 1999, S. 386.
} 


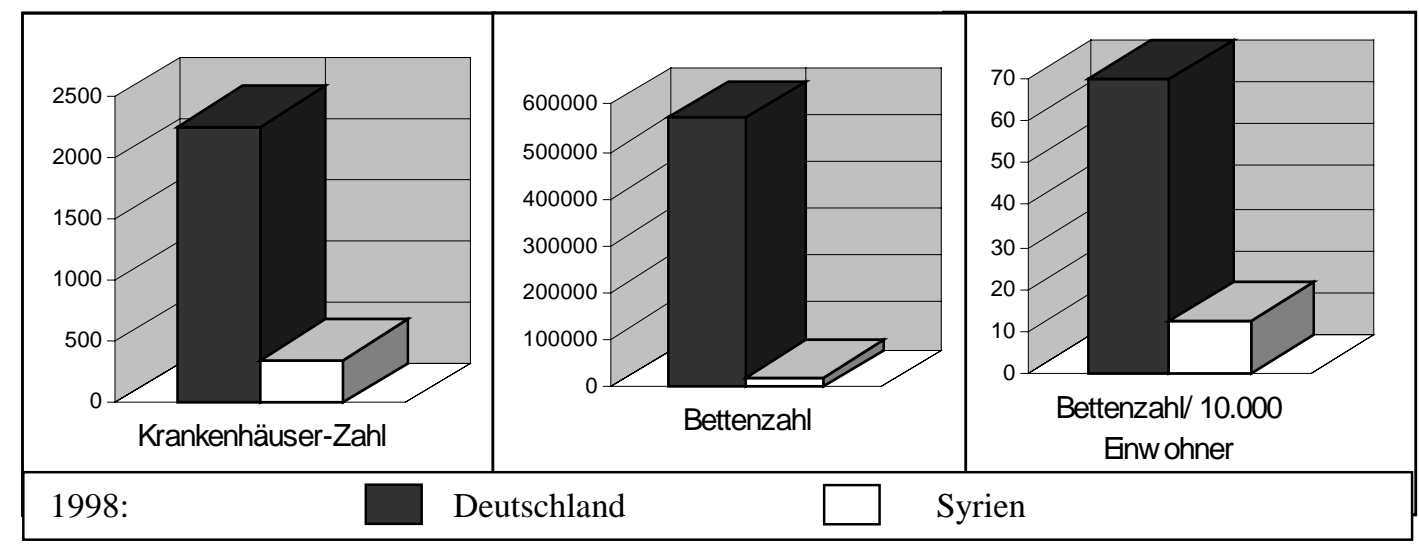

Abb. 35: Krankenhäuser- und Bettenzahlen Deutschland und Syrien.

Die Pflegesatzkosten betrugen 1998 in Deutschland 99,6 Mrd. DM und in Syrien 4,7 Mrd. DM (4,7 \%). Die Pflegesatzkosten pro Einwohner lagen 1997 in Deutschland 1.213,8 DM und in Syrien 324,1 DM (26,7 \%). Die Pflegesatzkosten pro Pflegetag betrugen 1998 in Deutschland 568 DM und in Syrien 94,6 DM (16.7\%) (Vgl. Abb. 36). ${ }^{485}$ Auch hier spiegelt sich die unterschiedliche allgemeine wirtschaftliche Lage der beiden Länder wieder.

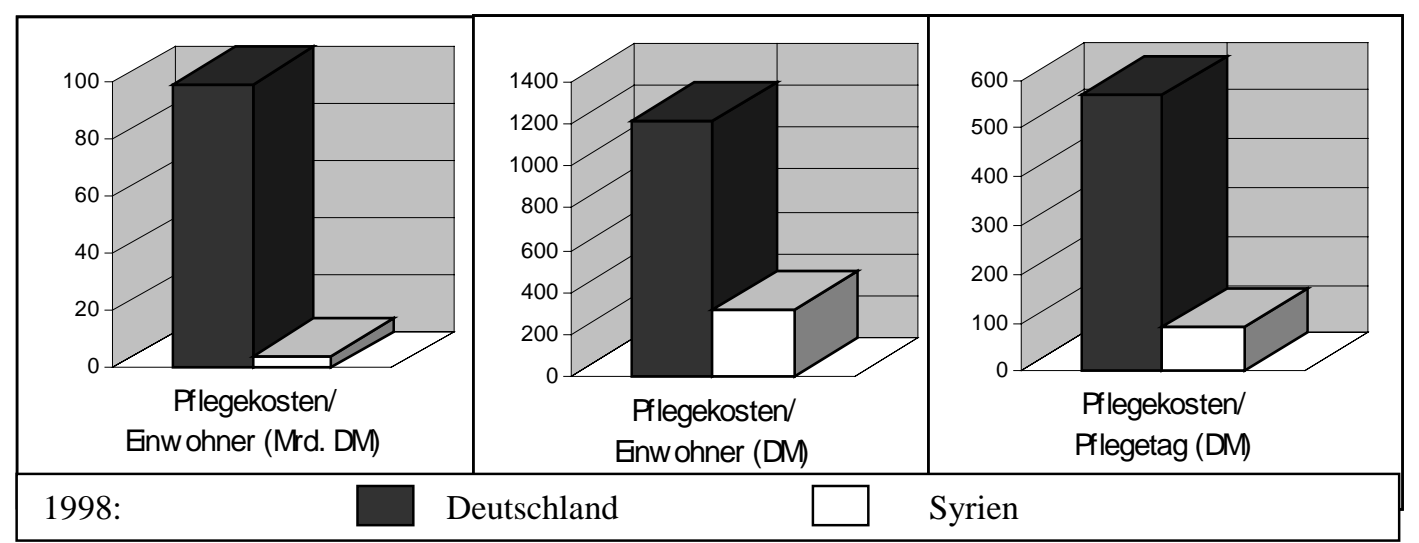

Abb. 36: Pflegekosten in Deutschland und Syrien.

Schließlich sollen zwei Universitätskliniken miteinander verglichen werden,. Dabei werden das Uni-Klinikum Göttingen und das syrischen Uni-Klinikum Damaskus herangezogen. 1997 gab es im Uni-Klinikum Göttingen 1.424 Betten und im UniKlinikum Damaskus 555 Betten. Die Gesamtkosten betrugen 1997 im UniKlinikum Göttingen 680 Mio. DM und im Uni-Klinikum Damaskus 11,8 Mil. DM $(1,7 \%)$. Entsprechend ergaben sich Gesamtkosten pro Bett in Uni-Klinikum

$\overline{485}$ Quelle: Vgl. Statistisches Bundesamt (1999), S. 2. 
Göttingen von 477,500 DM und im Uni- Klinik Damaskus von 21.100 DM (4,4 \%) (Vgl. Abb. 37). ${ }^{486}$

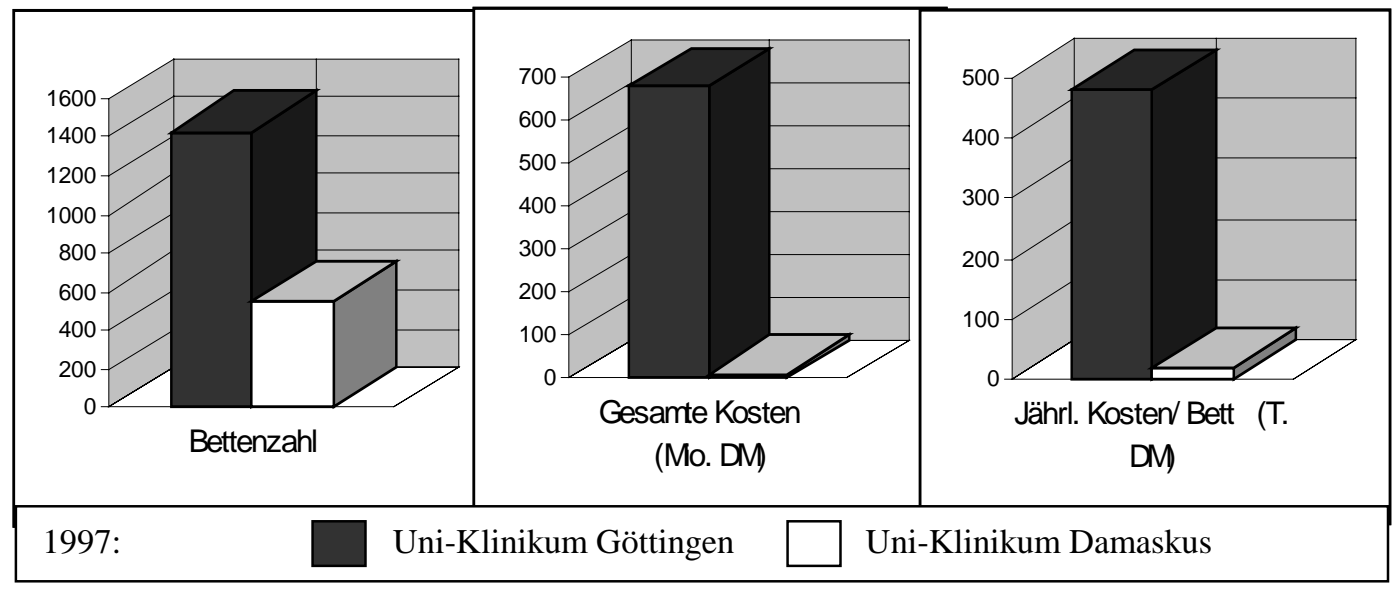

Abb. 37: Vergleich der Universitätsklinken Göttingen und Damaskus.

Zusammen fassend lässt sich bei diesem Vergleich feststellen, dass ein sehr großer Unterschied sowohl zwischen der allgemeinen Wirtschaftslage als auch den Gesundheits- und Krankenhaussituationen in Deutschland und Syrien besteht, der als ein Grund für den Unterschied zwischen dem Entwicklungsniveau des Krankenhaus-Managements in den beiden Ländern gesehen werden kann.

\subsection{Die Notwendigkeit einer Kosten- und Leistungsrechnung sowie eines Kostenmanagements im Krankenhaus}

Obwohl das betriebliche Rechnungswesen der meisten Krankenhäuser weniger von den Benutzern selbst auf der Grundlage ihrer selbst erkannten Informationsbedürfnisse entwickelt ist, sondern vom Gesetz- und Verordnungsgeber verfügt wurden, ist für einen Krankenhausbetrieb, der als Unternehmen nach dem Marktund Wirtschaftlichkeitsprinzip arbeiten soll, eine Kosten- und Leistungsrechnung unverzichtbar.

Krankenhäuser, die bisher keine Kosten- und Leistungsrechnung eingeführt haben, sollten sie realisieren, da eine Wirtschaftlichkeitskontrolle zunehmend erforderlich ist. Auf Grund der rückläufigen stationären Erträge und des zunehmenden Kostendrucks (Rentabilitäts-Aspekt).

\footnotetext{
486 Quelle: Klinikum der Georg-August-Universität Göttingen, Geschäftsbericht, 1997, S. 1 und 68, sowie Universitätsklinik „Assad“ Damaskus, Jahres Bericht 1997, Damaskus 4/ 1998, S. 5-8.
} 
Es reicht allerdings nicht aus, die Kosten- und Leistungsrechnung nur von außen zu entwickeln und sie nur als eine vornehmlich an externen Zwecken orientierte, arbeitsaufwendige Anordnung verstanden wird. Die Kosten- und Leistungsrechnung muss nach dem Krankenhaus-Bedarf entwickelt werden, womit seine Möglichkeiten als wertvolles internes Dispositions- und Controllinginstrument genutzt werden. ${ }^{487}$

Ein wichtiges Medium zur Durchführung und Entwicklung der Kosten- und Leistungsrechnung im Krankenhaus ist ein konsequentes Kostenmanagement sowie ein konsequentes Controlling-Management. Ein ausgebautes Controlling- und Kostenmanagement mit einer Budgetierung der Kosten, Leistungen und Erträgen ist ein wesentlicher Garant, um eine akute Krise - und darin befinden sich angesichts der gegebenen finanziellen Rahmenbedingungen praktisch alle Krankenhäuser - beherrschen zu können. ${ }^{488}$

Eine der Besonderheiten des Leistungserstellungsprozesses im Krankenhaus ist, dass die Leistungsmengen (Art und Anzahl der zu behandelnden Patienten) sowie die Erlöse je Leistung und Periode meistens extern festgelegt werden. Die Handlungsfreiheit zur Auswahl von kostengünstigen und/oder ertragsstarken Krankheitsarten und Therapien durch das Krankenhaus-Management ist hiermit eingeschränkt. Somit verstärkt sich die Notwendigkeit zur Einführung des Kostenmanagements im Krankenhaus.

Grundsätzlich ist das Kostenmanagement im Krankenhaus ein Hilfsmittel zur Ermittlung der Kostentransparenz bei der Leistungserstellung, zur Ermittlung der Kosteneinflussfaktoren, zur Ermittlung der Auswirkungen der Kosteneinflussfaktoren auf die kurzfristige und langfristige Kostenstruktur des Krankenhauses und zur Erarbeitung von Handlungsalternativen zur Kostenreduktion. ${ }^{489}$

Eingehend legt das strategische Kostenmanagement darauf aufbauend Maßnahmen zur zukünftigen Optimierung der Kostenstrukturen dar. Dadurch sind die technologischen Voraussetzungen, gesellschafts- und gesetzespolitischen Ent-

\footnotetext{
487 Vgl. Der Bundesminister für Arbeit und Sozialordnung (1984): Einsatz der Kosten- und Leistungsrechnung nach $\S 8 \mathrm{KHBV}$ für Analyse und Entscheidungen im Krankenhaus, Bonn, 1984, S.4.

${ }^{488}$ Vgl. Müller-Bellingroth, T. (1997),. S. 13-15.

${ }^{489}$ Vgl. Maltry, H., Strehlau-Schwoll, H. (1997), S. 554.
} 
wicklungen und demographischen sowie epidemiologischen Entwicklungen zu beachten, da sie e Erbringung der Krankenhausleistung (Leistungsprogramm) beeinflussen. Die Erkenntnisse dieser Beobachtungen führen $\mathrm{zu}$ einer strategischen Leistungsprogrammplanung, die im Krankenhaus die Voraussetzung für ein angemessenes Kostenmanagement ist.

Durch Prozess-Kostenmanagement wird eine eindeutige Dokumentation der Leistungserstellung durchgeführt, um anhand von sachbezogenen Auswertungen und von Expertenmeinungen eine Standardisierung der einzelnen Leistungen zu erreichen. Hierbei wird der Leistungserstellungsprozess (Aufnahme, Diagnostik, Therapie, Pflege und Entlassung) analysiert mit dem Ziel einer verbesserten Formulierung des Leistungserstellungsprozesses, womit unbenötigte Teilleistungen eliminiert werden können. ${ }^{490}$

In dem operativen Kostenmanagements sollen die Erstellungskosten je Leistung innerhalb bestehender Strukturen und bestehender Leistungsprogramme durch geeignete Maßnahmen reduziert werden. Hierbei bildet die strukturellen Zusammensetzung der Gesamtkosten, die für die Betrachtung nach unterschiedlichen Kriterien differenziert werden, den Ansatzpunkt für die Optimierung von Kostenstrukturen. Mit der Analyse des Verhältnisses der Kostenbestandteile können denn Unwirtschaftlichkeiten eliminiert werden. ${ }^{491}$

Im Folgenden werden die nach den Kostenarten analysierten Kostenstrukturen der deutschen Krankenhäuser, der Uni-Klinik Göttingen und der Uni-Klinik Damaskus des Jahres 1997 dargestellt (Vgl. Abb. 38).

\footnotetext{
${ }^{490}$ Vgl. ebenda S. 556.

491 Ein weiteres Beispiel ist die Darstellung der lebenszyklusspezifischen Kostenstruktur (die Relation von Vorlaufkosten, Herstell- und Vertriebskosten sowie Nachlaufkosten) allerdings ist dies im Krankenhaus nicht relevant. Vgl. Preuß, O. (1996), S., 94.
} 


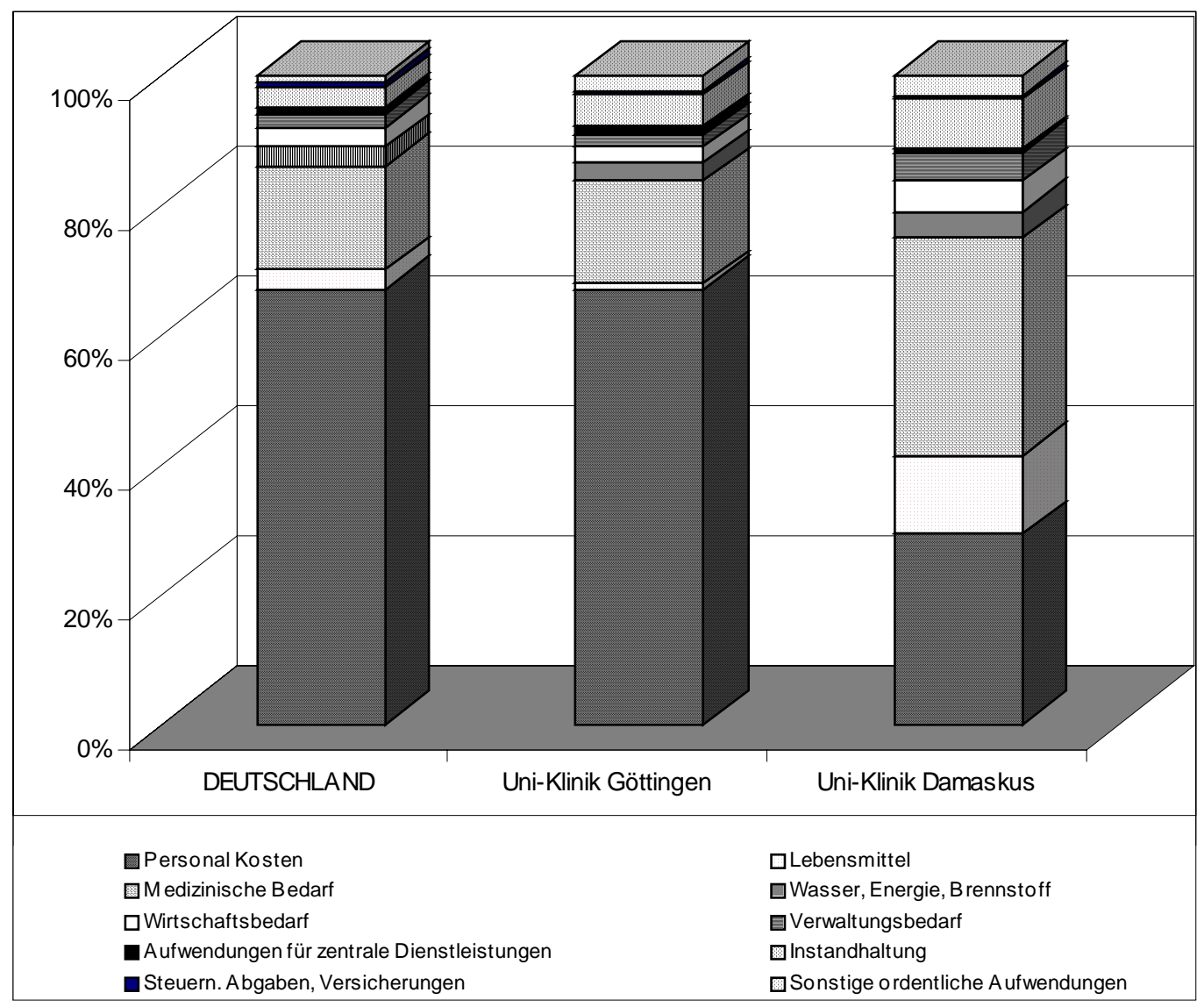

Abb. 38: Kostenartenstruktur des Krankenhauses ${ }^{492}$

Betrachtet man die Kostenstruktur der deutschen Krankenhäuser, so ist festzustellen, dass die Personalkosten den größten Anteil (67 \%) der gesamten Kosten haben. Als Ursache ist hier zu sehen, dass im Allgemeinen die Bereitschaftskosten in Krankenhäusern sehr hoch sind. Besonders in Deutschland zeigt sich diese Bereitschaft im Notfall- und Ambulanz-Bereich, die neben den normalen Behandlungs- und Therapiebereichen eine hoch Leistungsqualität haben. Da einer die Aufgaben der deutschen Krankenhäuser von außen vorgegeben sind, hat das Krankenhausmanagement wenig Einfluss auf die Leistungsarten, Leistungsmengen oder Leistungsqualität. Deswegen ist eine zentrale Aufgabe des Krankenhausmanagements die Kontrolle der Gemeinkosten. Durch ein Prozesskostenmanagement können die Notwendigkeit der einzelnen Teilleistungen

\footnotetext{
492 Quelle: Klinikum der Georg-August-Universität Göttingen: Geschäftsbericht, 1997, S. 68- 69, und Klinikum „Assad“ der Universität Damaskus: Jahres Bericht 1997, Damaskus 4/ 1998, S. 1011.
} 
hintergefragt werden, und möglicherweise Leerzeiten im Laufe der Behandlung kontrolliert werden, womit dieser Kostenanteil aber auch andern reduziert werden kann. $^{493}$

Die Personalkosten im Uni-Klinikum Damaskus betragen hingegen nur 29,3\% der Gesamtkosten. Sie betragen damit relativ gesehen weniger als die Hälfte im Vergleich mit Uni-Klinikum Göttingen. Die Ursache liegt hierin, dass generell die Löhne und Gehälter in Syrien viel niedriger sind als in Deutschland. Das Gehalt eines Arztes in Syrien ist nur ca. 15\% in gegenüber einem Arzt in Deutschland. Hingegen ist der Anteil des medizinischen Bedarfs relativ gesehen größer, da die meistens Artikel nach Syrien importiert und mit internationalen Preisen und Importkosten kalkuliert werden. Der Vorteil der geringeren Personalkosten in syrischen Krankenhäusern wird dadurch aufgehoben, dass das Krankenhausmanagement wenig Ersatzmöglichkeiten hat, auf Grund der Besonderheit des Leistungsprozesses in Krankenhaus die anderen Produktionsfaktor (z. B. Maschinen) durch die Arbeit des Personals zu ersetzen. ${ }^{494}$

\subsection{Betrachtung des Krankenhausbetriebs in Deutschland und Beurteilung seiner Kosten- und Leistungsrechnung}

Die Kosten- und Leistungsrechnung in deutschen Krankenhäusern ist grundsätzlich im Rahmen der krankenhausbezogenen Gesetze vorhanden gewesen. Sie wird bislang hauptsächlich von den externen Zwecken geprägt, die vor allem durch den Gesetzgeber vorgegeben werden. Die gesetzlichen Rahmenbedingungen der deutschen Krankenhäuser haben das Krankenhaus und seine Kosten- und Leistungsrechnung sowie das Rechnungssystem sowohl positiv als auch negativ beeinflusst.

1. Positive Betrachtungen an den Einflu $\beta$ der Rechtlichen Rahmenbedingungen: Im Allgemeinen hat sich das Krankenhaus unter den Vorgaben des KHG von der föderalistischen, staatlichen Krankenhausplanung (Kapazitätsplanung) über eine parallele Zuständigkeit zugunsten einer gemeinsamen Selbstverwaltung (Leistungsplanung) bis hin $\mathrm{zu}$ einer zentralstaatlich gelenkten Selbst-

493 Vgl. Hoffmann, J.: Personalkosten reduzieren statt Mitarbeiter entlassen, in: F\&W, 6/1999, S. 522- 523 . 
verwaltungslösung umgestellt. Die Selbstverwaltung wird somit namentlich auf ein Ausführungsorgan mit sozialpolitischen, staatlichen, aber auch finanzwirtschaftlichen und volkswirtschaftlichen Zielen hinauslaufen. ${ }^{495}$

Das gesetzlich vorgesehene Entgeltssystem für deutsche Krankenhäuser dient in seiner Entwicklung das durch Wettbewerbsprinzip der Erhöhung der Wirtschaftlichkeit des Krankenhauses. Die Stimulanz des Gewinns wird benutzt, um unter den Gegebenheiten der BPflV und des KHG die Wirtschaftlichkeit des Krankenhauses zu verbessern. Das Krankenhaus hat künftig verschiedene Unternehmensbereiche, in denen Gewinne oder Verluste entstehen können. Mit der Einführung von wettbewerbspolitischen Anreizmechanismen werden in Form von Festpreisen für genau definierte Leistungen zukünftig entscheidungsorientierte Führungs- und Steuerungsinformation $\mathrm{zu}$ stellen sein, mit denen eine langfristige Existenz des Krankenhauses durch das Erschließen von Wettbewerbsvorteilen gesichert werden soll. ${ }^{496}$ Es ist dann eine Aufgabe des Krankenhausmanagements, im Rahmen einer Unternehmensplanung personelle Entscheidungen $\mathrm{zu}$ treffen, vorsorglich und aktiv das Leistungsspektrum so abzugrenzen und zu planen, um das Krankenhaus nachhaltig wirtschaftlich zu sichern. Durch die Einführung von Festpreisen entsteht für Krankenhäuser ein dinglicher Anreiz, die Kosten pro Behandlungsfall so limitiert wie möglich zu halten, indem Einsparungspotentiale identifiziert werden und durch Leistungsausweitungen Degressionseffekte einsetzen können. ${ }^{497}$

Aus Rechnungsgesichtspunkten waren die gesetzlichen Rahmenbedingungen der stärkste Anlass überhaupt für eine Durchführung der Kosten- und Leistungsrechnung in den meisten deutschen Krankenhäuser, wodurch die Informationsstand für interne Kostenanalyse erheblich verbessert wurde. ${ }^{498}$ Die KHBV hat die Krankenhäuser auch bislang bereits verpflichtet, eine Kostenund Leistungsrechnung durchzuführen, welche die betriebsinterne Steuerung,

\footnotetext{
${ }^{494}$ Vgl. Abschnitt 2.1.3.

495 Vgl. Bruckenberger, E. (1993), S. 77.

496 Vgl. Menze, T. (1996), S. 200.

497 Die mit der Einführung differenzierter Entgeltformen verbundenen Zielsetzungen des Gesetzgebers betreffen aber mit eingesetzten Qualitätssicherungsmaßnahmen insbesondere die leistungsgerechte Belastung der Patient und ihrer Kostenträger (Krankenkassen). Vgl. Abschnitt 3.2.1.2.

${ }^{498}$ Vgl. Hippler, S.: Modulare Kostengliederung, in: F\&W, 1/1999, S. 47.
} 
die Beurteilung der Wirtschaftlichkeit und Leistungsfähigkeit, die Ermittlung der Selbstkosten und die Erstellung der relevanten Unterlagen für die Verhandlungen mit den Krankenkassen erlaubt. Die KHBV verlangte ferner, daß die Kosten und Leistungen verursachungsgerecht nach Kostenstellen zu erfassen und den anfordernden Kostenstellen zuzuordnen sind. ${ }^{499}$

Die Erhöhung der Kostentransparenz im Krankenhausbereich und die Vergleichbarkeit von Entgeltbeträgen mit anderen Krankenhäusern ist ein positiver Einfluss der rechtlichen Rahmenbedingungen, ${ }^{500}$ wobei ist es $\mathrm{zu}$ sehen, daß der deutsche Gesetzgeber im Rahmen der Reform des Gesundheitswesens auch die regelmäßige Durchführung eines Krankenhausvergleichs beschlossen hat. ${ }^{501}$

2. Negative Betrachtungen an den Einflu $\beta$ der Rechtlichen Rahmenbedingungen: Obwohl die rechtlichen Rahmenbedingungen bei der Einführung der Kostenund Leistungsrechnung in deutschen Krankenhäusern eine große Rolle gespielt haben, sind sie nachher eine Beschränkung der weiteren Entwicklungen. Ein deutliches Beispiel ist das verwendete Rechnungssystem. Da für das Krankenhaus die Vollkostenrechnung ${ }^{502}$ und damit eine Kalkulation auf VollkostenBasis gesetzlich vorgeschrieben ist, arbeitet die Mehrzahl der Krankenhäuser mit diesem traditionellen System. Aber da die traditionelle Vollkostenrechnung zum internen Zwecke der innerbetrieblichen Entscheidungen nicht genügt und da es kein gesetzliche Verbot zur Durchführung eines anderen Kostensystems gibt, empfiehlt es sich parallel dazu eine Teilkostenrechnung durchzuführen (Verfahren der Parallel- oder Doppelkalkulation). Im übrigen muss für externe Zwecke die Kostenrechnung auf Grund des bisherigen Kostenstellenrahmens aufgrund der BPflV 1995 erweitert werden, da diese zur Feststellung der Kosten und Leistungen unter den Anforderung des neuen Entgeltssystems (Ermöglichung der prospektiven Bildung der Abteilungspflegesätze und des

\footnotetext{
${ }^{499}$ Vgl. Tuschen, K.H., Philippi, M. (1995), S. 292.

500 Vgl. Koch, J., Schüller, L.: Das Gesetz zur Kontrolle und Transparenz im Unternehmen Bereich, in: F\&W, 1/1999, S. 40.

501 Vgl. von Eiff, W., [1997], S. 613.

502 Ebenfalls beim anderen Betrieben im öffentlichen Bereich, in denen ,fast ausnahmslos Kostenrechnung im traditionellen Stil betrieben wird“"Vgl. Brede, H. (1997), S. 223.
} 
Basispflegesatzes zur Unterstützung der internen Budgetierung) nicht mehr geeignet ist.

Für die Zwecke der Preisermittlung nach den geltenden Vorschriften ist eine Vergütung nach dem Solidaritätsprinzip durchgeführt. Die Anwendung eine Äquivalenzziffernrechnung bei der Bewertung jeder einzelnen Leistung mit einer Punktzahl (nach dem Krankenhaustarif DKG-NT) bzw. Punktwert (nach GOÄ) in der GOÄ spiegelt die unterschiedlichen Durchschnittskosten wieder. Sie sind nicht nach kostenrechnerischen Aspekten, sondern als ,politische Preise“ vorgenommen worden. Daher entsprechen diese Leistungen nicht unbedingt den tatsächlich unter Berücksichtigung der Kostenstruktur des einzelnen Krankenhauses entstandenen Kosten der Einzelleistung. Deswegen müssen die Äquivalenzziffern in der Kostenrechnung in Krankenhäusern abhängig von dem betriebsindividuelle Leistungserstellungsverfahren und unter Berücksichtigung der Veränderung das Leistungsspektrums durch den medizinisch-technischen Fortschritt laufend weiter entwickelt werden. ${ }^{503}$

\subsection{Betrachtung und Empfehlungen für syrische Krankenhäuser}

Eine Modifizierung des Managements eines Krankenhauses ist kein isolierter Prozess. Er hängt von verschiedenen Handlungsfeldern $a b,{ }^{504}$ vor allem sind das Entwicklungsniveau der Wirtschaftswissenschaft und das Ausbildungssystem zu nennen. Das syrischen Ausbildungssystem muss für die Modernisierung und Liberalisierung der Wirtschaft neu diskutiert werden. Darüber hinaus ist die Qualifizierung der Verwaltungskräfte und Erhöhung der Kostenbewusstsein der Mitarbeiter eine Voraussetzung für diesen Prozess, da die Krankenhaus-Mitarbeiter ebenfalls ein zu betrachtender Faktor sind, ${ }^{505}$ hierzu ist eine ständige Weiterbildung auch erforderlich. Hierbei sind insbesondere das Kostenbewusstsein und die Ausbildung in Kostenrechnung von großer Bedeutung, da „die Kostenrechnung in allen wirtschaftsorientierten Ausbildungsgängen eine große Rolle spielt'“506.

\footnotetext{
${ }^{503}$ Vgl. Deutsche Krankenhausgesellschaft (1992), S. 182.

504 Vgl. Trill, R. (2000), S. 20.

505 Finkler, S.: The Human Element in Cost Accounting, in: Finkler, S. A. (Edit.), Issues in Cost Accounting for Health Care Organisations, New York, 1994, S. 15.

506 Bloech, J.: Kostenmanagement in Planspielen, in: in: Freidank, C., Götze, U., Huch, B., Lueter, G., (Hrsg.): Kostenmanagement, Heidelberg, 1997, S.382.
} 


\subsubsection{Modernes Management im syrischen Krankenhaus}

Das Krankenhaus in Syrien soll vor allem als wirtschaftlicher Betrieb betrachtet werden Und sollte nach betriebswirtschaftlichen Aspekten (Wirtschaftlichkeits-, Markt- und Rentabilitätsprinzipien) geleitet werden. Die Stärkung der eigenen Wettbewerbssituation sollte Anliegen Nr. 1 in den syrischen Krankenhäusern sein, um erfolgreich am Markt bestehen zu können. Die Regierung könnte zugleich ihr Ziel verfolgen, daß die Gesundheit der Bevölkerung eine der zentralen Aufgaben des Staats ist. Das dualistische Finanzierungssystem in Deutschland könnte dabei als achtbares Beispiel dienen. Die syrische Regierung könnte die Investitionskosten der Krankenhäuser übernehmen und daneben neue aktive Institutionen (gesetzliche staatliche Krankenkassen) schaffen, die die Betriebskosten übernehmen können. Damit werden die Leistungen der Krankenhäuser nicht mehr kostenlos, sondern vergütete Leistungen. Das Krankenhaus-Management kann und soll in dieser Weise die Kosten und Leistungen nach wirtschaftlichen Aspekten betrachten. Hierbei sollte nicht vergessen werden, gleichzeitig ausreichende Verordnungen für die Qualitätssicherung der Krankenhausleistungen schaffen.

Für das moderne Management im syrischen Krankenhaus ist die EDV wie auch in industriellen Betrieben ein wichtiges Hilfsmittel, besonders bei der Erfüllung der Aufgabe der Datenversorgung für zentrale Einrichtungen. Somit könnte die Kosten- und Leistungsrechnung nach krankenhaus-individuellen Gesichtspunkten durchgeführt werden, wobei die Daten für diese Einrichtungen daneben mit Hilfe der EDV genau und schnell erfasst und bearbeitet werden können.

\subsubsection{Aufbau- und Übertragungsmöglichkeiten einer Kosten- und Leistungs- rechnung}

Der Aufbau einer Kosten- und Leistungsrechnung in syrischen Krankenhäusern ist zwingende Voraussetzung für die zukünftige Steuerung jedes Krankenhauses. Vielmehr ist davon auszugehen, dass Krankenhäuser, die nicht kurzfristig eine Kosten- und Leistungsrechnung einführen und ein entsprechendes Kostenmanagement betreiben, nicht fähig sein werden, die vom Staat erwarteten Aufgaben zu erfüllen. 
Der Aufbau einer Kosten- und Leistungsrechnung in syrischen Krankenhäusern muss allerdings die Gegebenheiten der syrischen Wirtschaft betrachten. Eine wichtige von ihnen ist das Syrischen-Einheitlichen-Rechnungs-System (SERS) als Gesetz für die Kosten- und Leistungsrechnung. Grundsätzlich ist der Aufbau einer Kosten- und Leistungsrechnung in syrische Krankenhäuser anhand der Bestimmungen des SERS möglich. Es gilt aber, bewährte Verfahren und Methoden der Kosten- und Leistungsrechnungssysteme auf die Besonderheiten des Krankenhauses zu adaptieren und zu implementieren. Allerdings könnte auf Grund langjähriger Erfahrungen industrieller Unternehmen der Aufbau einer krankenhausspezifischen Kosten- und Leistungsrechnung von den dortigen Erfolgen und Misserfolgen profitieren. Eine sinnvolle Adaption empfiehlt sich wegen der besonderen Verhältnisse im Krankenhaus.

Auch die Erfahrungen bei der Durchführung und Entwicklung einer spezifischen Kosten- und Leistungsrechnung in Krankenhäusern anderer Länder sind zu beachten. Die Übertragung der in deutschen Krankenhäusern durchgeführten Kostenrechnung kann wie folgt vorgenommen werden.

Auf Grund der Ähnlichkeit der Kostenarten in allen Krankenhäusern, wobei die Leistungen der Krankenhäuser nach den medizinischen Lehren und Techniken erstellt werden, ist die Übertragbarkeit in Kostenartenrechnung möglich. Nur ist zu beachtet, daß die Kostenarten anhand der Kontenrahmen des SERS neu kodiert werden sollten. ${ }^{507}$

Die Kostenstellenrechnung als wesentliches Element der Kostenrechnung sollte aufbau- und ablauforganisatorisch durchgeführt werden, wobei die individuellen Einrichtungen entscheidend von der Größe und Struktur des Krankenhauses abhängig sind. Aber auch hier macht die Ähnlichkeit des Krankenhaus-Prozesses möglich, dass die in deutschen Krankenhäusern durchgeführte Kostenstellenrechnung beim Aufbau einer Kostenstellenrechnung für syrische Krankenhäuser übertragen werden kann. Allerdings ist eine Umkodierung nach dem im SERS vorgesehenen Kontenrahmen erforderlich. ${ }^{508}$

Wegen der Besonderheiten des Finanzierungssystems, nach dem die Kostenträgerrechnung in deutschen Krankenhäusern durchgeführt wird, ist die Übertragung des

${ }^{507}$ Vgl. Abschnitt 4.3.1.2. 
deutschen Systems in der Kostenträgerrechnung behindert. In syrischen Krankenhäusern könnte bis zur Einführung eines neuen Finanzierungssystems der PatientFall als Kostenträger betrachtet werden.

Das Kostenmanagement stellt hierauf aufbauend lediglich eine Folgemaßnahme dar, indem die Erkenntnisse der Kosten- und Leistungsrechnung zielgerichtet umgesetzt werden. Abschließend kann festgehalten werden, dass die Erstellung der krankenhausspezifischen Dienstleistungen die Artwendungen von betriebswirtschaftlichen Erkenntnissen wie sie bereits aus der Industrie bekannt sind, weder ausschließt, noch überflüssig macht.

${ }^{508}$ Vgl. Abschnitt 4.3.2.1. 


\section{Literaturverzeichnis}

Adam, D. (1972): Krankenhausmanagement im Konfliktfeld zwischen medizinischen und wirtschaftlichen Zielen, Wiesbaden 1972.

Adam, D. (1996): Krankenhausmanagement im Wandel. In: Adam, D. (Hrsg.): Krankenhausmanagement. Schriftenreihe zur Unternehmensführung (SzU), Band 59, Wiesbaden, 1996.

Aldag, H. (1988): Rahmenbedingungen, Problemlösungserfordernisse und konzeptionelle Grundlegung eines Marketing für Krankenhäuser, Dissertation, Göttingen, 1988.

American Hospital Association (1974): classification of Health Care Institutions, Chicago: AMA, 1974.

Andersch, N. (1992): Krankenhausentwicklung und gewerkschaftliche Krankenhauspolitik: Eine Übersicht unter besonderer Berücksichtigung der ÖTV., Va\&g, Marburg, 1990.

Andersen, H., Henke, K., Schulenberg, M. (1992): Basiswissen Gesundheitsökonomie, Band I, Einführende Texte, Berlin, 1992.

Arbeitsgruppe „Entgeltsysteme“ in Bundesgesundheitsministerium (Hrsg.): Grundkonzeption eines neuen Entgeltsystems, Bonn, 1993.

Arnold, M. (1988): Möglichkeiten der Verzahnung des ambulanten und stationären Sektors, in: das Krankenhaus, 3/1988, S. 98- 103.

Baschke, W. L. (1994): BPfIV 95- der Kompromiß, in: F\&W, 4/1994.

Baugut, G., Schmitz, R. (1993): Methodik zur Kalkulation hausindividueller Fallkosten für den Vergleich mit standardisierten Fallpauschalen, in: das Krankenhaus, 4/ 1993, S. 170- 174.

Baugut, G. (1995): Handlungshinweise zur Kalkulation von Fallpauschalen und Sonderentgelten, in: Eichhorn, S. (Hrsg.): Krankenhausmanagement im Werte- und Strukturwandel, Stuttgart, Berlin, Köln, 1995, S. 145- 170. 
Berghöfer, B. (1997): Profit- Center als Organisationsform im Krankenhaus, Fachaufsatz des Klinikums der Phillips- Universität, 1997.

Bloech, J., Götze, U., Sierke, B. (1993): Managementorientiertes Rechnungswesen: Konzepte und Analysen zur Entscheidungsvorbereitung, Wiesbaden, 1993.

Bloech, J. (1997): Kostenmanagement in Planspielen, in: Freidank, C., Götze, U., Huch, B., Weber, J. (Hrsg.): Kostenmanagement, Aktuelle Konzepte und Anwendungen, Berlin, Heidelberg: Springer- Verlag, 1997, S.379-399.

Bölke, G. (1987): Einführung mit der neuen Bundespflegesatzverordnung, in: das Krankenhaus, 6/ 1987, S. 214- 219.

Bölke, G., Schmidt- Rettig, B. (1988): Leistungsrechnung- Leistungsstatistik: in Eichhorn, S. (Hrsg.), Handbuch Krankenhausrechnungswesen - GrundlagenVerfahren- Anwendungen, 2. Aufl., Wiesbaden, 1988.

Bopp, Manfred, Hohenbild, Rolf (1984): Kosten- und Leistungsrechnung im Krankenhaus, Köln 1984.

Brayer, F. (1991): Die Kalkulation von Preisen im Krankenhauswesen - Ziele, Methoden und Auswirkungen, Heidelberg, 1991.

Brede, H. (1997): Betriebswirtschaftslehre, Einführung, 6., unwesentl. veränd. Aufl., München, Wien, 1997.

Bruckenberger, E. (1993): von der Länderkompetenz zum „Einkaufsmodell“ der Krankenkassen“- die Konsequenzen des Gesundheits-Strukturgesetzes für Krankenhäuser, in: das Krankenhaus, 2/ 1993, S. 77-85.

Bundesministerium für Gesundheit (1993): Grundkonzeption des neuen Entgeltsystems. Bonn, 1993.

Bundesministerium für Gesundheit (1995 a): Leitfaden zur Einführung von Fallpauschalen und Sonderentgelten gemäß BPflV 1995: Datenbedarf, Kalkulationsgrundlagen, Abrechnungsmodalitäten, Kostengliederung, Schriftenreihe des Bundesministeriums für Gesundheit, Band 44, Baaden-Baaden, 1995.

Bundesministerium für Gesundheit (1995 b): Kalkulation von Fallpauschale und Sonderentgelten gemäß Bundespflegesatzverordnung 1995: Bericht zu den 
Forschungsprojekt, im Auftrag für Bundesministerium für Gesundheit, Band 45, Baden-Baden, 1995.

Chan Yee-Ching, L. (1994): Improving Hospital Cost Accounting with ActivityBased Costing, in: Finkler, S. A. (Edit.), Issues in Cost Accounting for Health Care Organisations, New York, 1994, S. 261-267.

Coenenberg, A. (1992): Kostenrechnung und Kostenanalyse, Landsberg, Lech, 1992.

Conrad, H., J. (1997): Anforderung an ein leistungsfähiges Krankenhausmanagement, Profit- Center- Steuerung durch dreistufige Deckungsbeitragsrechnung, in: das Krankenhaus, 10/ 1997, S. 607- 610.

Davis Weintraub, L., Dube, R. J. (1994): Alternative Costing Methods in Health Care, in: Finkler, S. A. (Edit.),Issues in Cost Accounting for Health Care Organisations, New York, 1994 , S. 114-118.

Der Bundesminister für Arbeit und Sozialordnung (1984): Einsatz der Kostenund Leistungsrechnung nach $§ 8$ KHBV für Analysen und Entscheidungen im Krankenhaus, Bonn, 5/ 1984.

Deutsche Krankenhausgesellschaft (1990): Auswertung von Kosten- und Leistungsnachweisen 1988, Düsseldorf, 1990.

Deutsch Krankenhausgesellschaft (1993): Sonderentgelte und Fallpauschalen gefährden die bürgernahe Krankenhausversorgung- Stellungnahme der DKG zur „Grundkonzeption des neuen Entgeltssystems“ des Bundesministeriums für Gesundheit, in das Krankenhaus, 4/1993. S. 155- 158.

Deutsche Krankenhausgesellschaft (1994 a): Positionen der DKG zur Reformdiskussion im Gesundheitswesen, Die Stellung des Krankenhauses in der künftigen gesundheitlichen Versorgung, in: Das Krankenhaus, 12/1994, S. 538- 545.

Deutsche Krankenhausgesellschaft (1994 b): Hinweise der DKG zum Rechnungswesen der Krankenhäuser unter besonderer Berücksichtigung der Anpassung des Rechnungswesen der Krankenhäuser in der neuen Bundesländern an die Erfordernisse des Krankenhausfinanzierungsrechts, Düsseldorf, 1992.

Deutz, W. (1999): Marketing als Erfolgsfaktor in Krankenhausmanagement, Frankfurt a. M., Berlin, Neu York, Paris, Wien: Lang, 1999. 
Dieter, B. (1994): Understanding the Hospital Cost Accounting Process, in: Finkler, S. (Edit.), Issues in Cost Accounting for Health Care Organisations, New York, 1994, S. 8- 13.

Dietz, O., Bofinger, W. (1995): Krankenhausfinanzierungsgesetz, Bundespflegesatzverordnung und Folgerecht- Kommentare, Wiesbaden, 1995.

Ebert, G: Kosten- und Leistungsrechnung, 6. Aufl., Wiesbaden 1991.

Ehrlich, A. (1996): Neuorientierung des Krankenhauscontrolling, in: BiBu, 2/1996.

Eichhorn, Siegfried (1975): Krankenhausbetriebslehre - Theorie und Praxis des Krankenhausbetriebes, Band. I, 3. Aufl., Stuttgart, Köln, Berlin, Mainz, 1975.

Eichhorn, Siegfried (1976): Krankenhausbetriebslehre - Theorie und Praxis des Krankenhausbetriebes, Band. II, 3. Aufl., Stuttgart, 1976.

Eichhorn, Siegfried (1979): Betriebswirtschaftliche Ansätze zu einer Theorie des Krankenhauses, in: Zeitschrift für betriebswirtschaftliche Forschung, 3/ 1979.

Eichhorn, Siegfried (1987): Krankenhausbetriebslehre - Theorie und Praxis der Krankenhau- Leistungsrechnung, Kohlhammer(Hrsg.), Band. III, Köln, Stuttgart, Berlin, Mainz, 1987.

Eisele,W. (1980): Technik des betrieblichen Rechnungswesens, München, 1980.

Engelke, D., Riefenstahl, R. (1985): Die Auswirkung der Flexibilisierung des Krankenhausbudgets auf die Erlössituation und das Betriebsergebnis des Krankenhauses, in: Krankenhaus Unschau, 5/1985.

Finkler, S. (1994 a): Cost Accounting for Human Recourses in Hospitals, in: Finkler, S. A. (Edit.),Issues in Cost Accounting for Health Care Organisations, New York, 1994.

Finkler, S. (1994 b): The Human Element in Cost Accounting, in: Finkler, S. A. (Edit.),Issues in Cost Accounting for Health Care Organisations, New York, 1994.

Fischer, D. (1988): Marktstruktur und Marktverhalten in der Krankenhauswirtschaft, Spardorf, 1988.

Freidank, C. (1994): Kostenrechnung, Einführung in die begrifflichen, theoretischen, verrechnungs-technischen sowie planungs- und kontrollorientierten 
Grundlagen des innerbetrieblichen Rech-nungswesens, 5. Überarb. und erw. Aufl., München, Wien: Oldenburg, 1994.

Freymann, H. (1989): Controlling in Krankenhaus - Möglichkeiten und Nutzen für die Betriebsführung, in: Eichhorn, s., Feyermann, H., (Gesamtverantwortung, Wirtschaftliches Krankenhaus, Beiträge zu Management, Planung, Rechnungswesen, Prüfung, 3. Aufl., Köln, 1989.

Frömming, N. (1977): Management im Krankenhaus aus verhalteneswissenschaftlicher Sicht, Baden- Baden, 1977.

Fuchs, M. (1988): Grundlagen des betrieblichen Rechnungswesens, in: Eichhorn, S. (Hrsg.), Handbuch Krankenhaus - Rechnungswesen, 2. Aufl., Wiesbaden, 1988.

Gabele, E., Fischer, P. (1992): Kosten- und Erlösrechnung, München 1992.

Gebhard, W. (1986): Controlling im Krankenhaus, in: Controlling 6/ 1986, S. 207212.

Gerdelmann, W. (1996): GSG aus Sicht der Krankenkassen, in: Adam, D. (Hrsg.): Krankenhausmanagement, Band 59, SzU., Wiesbaden, 1996. S. 33- 48.

Götze, U., Meyerhoff, J. C. (1993): die Prozesskostenrechnung - Stand und Entwicklungstendenzen, in: Zeitschrift für Planung, 1/ 1993, S. 65- 96.

Götze, U.: Einsatzmöglichkeiten und Grenzen der Prozesskostenrechnung, in: Freidank, C., Götze, U., Huch, B., Weber, J. (Hrsg.): Kostenmanagement, Aktuelle Konzepte und Anwendungen, Berlin, Heidelberg: Springer- Verlag, 1997, S.141174.

Götzinger, M., Michael, H. (1990): Kosten- und Leistungsrechnung, eine Einführung, 5. Aufl., Heidelberg, 1990.

Graf, V. (1999): Prozessoptimierung im Krankenhaus, steigert die Effizienz und festigt die Kundenbindung, Erfahrungen aus dem Klinikum der Stadt Ludwigshafen, in: F\&W, 6/ 1999, S. 516- 521

Gronemann, J. (1988): Überlegungen zur Rechtsformwahl bei Krankenhäusern, in: Führen und Wirtschaften im Krankenhaus, Juni, 1988, S. 2 - 7. 
Grünenwald, K., Kehr, H., Tuschen, K. (1987): Kommentar Leistungs- und Leistungsnachweis der Krankenhäuser, Bad Homburg, 1987.

Haberstock, L. (1987): Kostenrechnung, Bd.1: Einführung mit Fragen, Aufgaben und Lösungen, 8. Aufl., Wiesbaden, 1987.

Haberstock, L. (1998): Kostenrechnung, Bd.1: Einführung mit Fragen, Aufgaben einer Fallstudie und Lösungen, 10. Aufl., Berlin, 1998.

Haubrock, M. (1994): Sachgütereinsatz im Krankenhaus, in: Peters, S. Schär, W. (Hrsg.): Betriebswirtschaft und Management im Krankenhaus, Berlin, 1994.

Hauschild, E., Schomacker, R., Strauf, H.G. (1981): Kriterienmodell zur Einordnung von Krankenhäusern in ein Abgestuftes Versorgungssystem, der Bundesminister für Arbeit und Sozialordnung (Hrsg.), Bonn, 1981.

Heinen, E. (1978): Betriebswirtschaftliche Kostenlehre, 5. Aufl., Wiesbaden, 1978.

Heintze, J., Kehres, E. (1995): Kosten- und Leistungsrechnung in Krankenhaus, systematische Einführung, 3. Aufl., Stuttgart, Berlin, Köln: Kohlhammer, 1995.

Hentze, J. (1979): Kosten- und Leistungsrechnung in Krankenhäuser systematische Einführung, Kohlhammer (Hrsg.) Köln, Stuttgart, Berlin, Mainz, 1979.

Herbold, W., Horstmann, P., Gemke,H. (1997): Leistungsstrukturveränderungen, Nullrunde oder Budgeterhöhung?, in: Das Krankenhaus, 7/ 1997, S. 531- 534.

Herder-Dorneich, Ph. (1981): Problemgeschichte der Gesundheitsökonomik, in: Herder-Dorneich, Sieben, Thiemeyer (Hrsg.), 1981.

Herder-Dorneich, P. (1985): Wettbewerb und Rationalitätsfalle im System der Gesetzlichen Krankenversicherung, in: Hamm, Neubauer (Hrsg.) 1985, S. 13-61.

Herder-Dorneich, PH., Wasem, J. (1986): Krankenhausökonomik zwischen Humanität und Wirtschaftlichkeit, Baden-Baden, 1986.

Hildebrand, R. (1988): Kostenrechnung, in: Eichhorn, S. (Hrsg.), Handbuch Krankenhausrechnungswesen, Grundlagen- Verfahren- Anwendungen, 2. Aufl., Wiesbaden, 1988, S. 343-456. 
Hörmann, W., Ingruber, H. (1988): Krankenhausbetriebslehre: Grundzüge der Betriebsführung im Krankenhaus, 1. Aufl., Wien, 1988.

Horvath, P., Mayer, R. (1989): Prozesskostenrechnung, Der neue Weg zu mehr Kostentransparenz und wirkungsvolleren Unternehmensstrategien, In: Controlling, 7/1989, S. 216-232.

Horvath, P., Mayer, R. (1995): Konzeption und Entwicklung der Prozesskostenrechnung. In: Mannel, W. (Hrsg.), Prozesskostenrechnung. Wiesbaden, 1995, S. 59- 86.

Hübner, H. (1980): Kostenrechnung im Krankenhaus, Grundlagen, Wirtschaftlichkeitsanalyse, Betriebsvergleich, 2. Aufl., Stuttgart, Berlin, Köln, Mainz, 1980.

Hummel, S., Männel, W. (1986): Kostenrechnung, Grundlagen, Aufbau und Anwendung, 4. Völlig neu und erweiterte Aufl., Wiesbaden, 1986.

Janssen, D. (1999): Wirtschaftlichkeitsbewertung von Krankenhäuser, Konzepte und Analysen von Betriebsvergleichen, Stuttgart, Berlin, Köln, 1999.

Jungmann- Ginkel, E., Kober, K. (1993): Krankenhausbetriebslehre für Führungskräfte der Krankenpflege, Lorsch, 1993.

Juretzka, G. (1989): Aufbau eines internen Rechnungswesens am Universitätsklinikum Göttingen, in: das Krankenhaus, 11/ 1989, S. 591-595.

Kaltenbach, T. (1993): Qualitätsmanagement in Krankenhaus, Qualitäts- und Effizienzsteigerung auf der Grundlage des Total Quality Management, 2. Aufl., Melsungen, 1993.

Kehres, E. (1990): Kosten und Kostendeckung der ambulanten Behandlung im Krankenhaus, Dissertation an der Uni. Braunschweig, 1990.

Keisers, J. (1979): Einführung in der Unternehmung, 2. Aufl., Wiesbaden, 1979.

Keun, F. (1997): Einführung in die Krankenhauskostenrechnung, 2. Aufl., Wiesbaden: Gabler, 1997.

Keun, F. (1999): Einführung in die Krankenhauskostenrechnung, 3. Überarbeitete Aufl., Wiesbaden: Gabler, 1999. 
King, M., Lapsly, I, Mitcbell, F., Moyes, J. (1994): Activity Based Costing in Hospital, A Case Study Investigation, The Chartered Institute of Management Accountants, London 1994.

Klask. J, Smerzer, A. (1997): Fallkostenkalkulation der Therapien Lonsillektomie und Septumplastik, Gesamtkosten unter Berücksichtigung typischer Komplikation, in: Deutschland Krankenhaus, 2/ 1997, S. 59-64.

Klinikum der Georg-August-Universität Göttingen (1997): Geschäftsbericht, Göttingen, 1997.

Klockhaus, H. E. (1997): Kosten- und Leistungsrechnung im Krankenhaus, München, Essen, 1997.

Kloock, J., Sieben, G., Schildbach, T. (1999): Kosten- und Leistungsrechnung, 8., aktualisierte und erw. Aufl., Düsseldorf, 1999.

Koch, J. (1998): Gesundheitsökonomie, Betriebswirtschaftliche Kosten- und Leistungsrechnung, München, Wien, Oldenburg, 1998.

Kolb, T. (1994): Prozesskostenrechnung im Krankenhaus, in: F\&W, 5/ 1994, S. 396- 401.

Kosiol, E. (1979, A): Kosten- und Leistungsrechnung, Berlin1979.

Kosiol, E. (1979, B): Kostenrechnung der Unternehmung, 2. Aufl., Wiesbaden, 1979.

Kropf, R.(1994): Physician Cost Variance Analysis under DRGs, in: Finkler, S. A. (Edit.),Issues in Cost Accounting for Health Care Organisations, New York, 1994.

Leonhardt, J. (1988): Aufbau- und Anwendungsmöglichkeiten einer Grenzplankostenrechnung in Krankenhausbetrieben, Hamburg, 1988.

Maltry, H., Strehlau-Schwoll, H. (1997): Kostenrechnung und Kostenmanagement im Krankenhaus, in: Freidank, C., Götze, U., Huch, B., Weber, J. (Hrsg.): Kostenmanagement, neue Konzepte und Anwendungen, Berlin, Heidelberg, 1997, S. 533- 567.

Menze, T., Michels, R. (1996): Controlling - Instrumente im Krankenhaus, Existenzsicherung durch mehr Wirtschaftlichkeit, in: F\&W, 3/1996, S. 200 - 206. 
Meyer, D. (1993): Technischer Fortschritt im Gesundheitswesen, Tübingen, 1993.

Mildner, R. (1987): Der Effizienzbegriff im Gesundheitswesen, in: Krankenhaus Umschau, August 1987, S. $664-669$.

Möhlmann, E. (1992): Neue Wege in der Leistungs- und Kostenrechnung, in: F\&W, 2/1992, S.87-92.

Monka, M. (1993): Entwicklung und Berechnung von Fallpauschalen, Das ökonomische Modell des WidO, in: Arnold, M., Paffrath, D., Krankenhaus-Report 1993, Aktuelle Beiträge, Trends, Statistik, Stuttgart, 1993.

Mooney, G. (1986): Economics, Medicine, and Health care, Sussex: Wheatsheaf Books, 1986.

Müller, H. (1988): Preisrecht und Gebührenwesen, in: Eichhorn, S. (Hrsg.), Handbuch Krankenhausrechnungswesen - Grundlagen- Verfahren- Anwendungen, 2. Aufl., Wiesbaden, 1988, S. 615-686.

Neubauer, G. (1998): Kriterien zur Bewertung und Auswahl eines KrankenhausVergütungssystems, in: das Krankenhaus, 10/ 1998, S. 578-581.

Neubauer, G. (1999): Formen der Vergütung von Krankenhäusern und deren Weiterentwicklung, in: Braun, G. (Hrsg.), Handbuch Krankenhausmanagement, Baustein für eine moderne Krankenhausführung, Stuttgart, 1999, S. 19- 35.

Neubauer, G., Zelle, B. (1996): Fallpauschalen: Ein Ansatz zu einer Leistungsbezogenen Krankenhausvergütung, in: Adam, D. (hrsg.): Krankenhausmanagement, Schriftenreihe zur Unternehmensführung (SzU.), Band 59, S. 19 - 32, Wiesbaden 1996.

O. V. (1993): Fallpauschalen, die Empfehlungen der Institute, in: F\&W, 10 Jahrgang, 1993, S.400-405.

Pfaff, M. (1995): Das Krankenhaus im Gefolge des Gesundheitsstrukturgesetzes 1993, Baden- Baden, 1995.

Poelling, S. (1996): The Role of the Private Sector in the Syrian Economy, in: Economic Liberalization and Privatization in Socialist Arab Countries,: Hopfinger, H., (Hrsg.), Gotha: Perthes, 1996. 
Preuß, O. (1994): Das Krankenhaus als Betrieb, in: Peters, S., Schär, W. (Hrsg.), Betriebswirtschaft und Management im Krankenhaus, S. 92 - 109, Berlin, 1994.

Preuß, O. (1996): Kosten und Deckungsbeitragsmanagement im Krankenhaus unter besonderer Berücksichtigung von Fallpauschalen und Sonderentgelten, Lang, P. (Hrsg.), Frankfurt am Main, Berlin, Bern, New York, Paris, Wien, 1996.

Pschyrembel, W. (1996): Klinisches Wörterbuch, 255. Aufl., Berlin, 1986.

Raem, A.: der Arzt als Manager, München, 1996.

Regler, K. (1994): Auswirkung des GSG und Handlungsbedarf der Krankenhausträger, in: Das Krankenhaus, 1/1994, S. 1-6.

Richter, H (1994).: Kostenträgerrechnung und interne Budgetierung ab 1995/1996, neue Formen der Kosten- und Leistungsplanung, in: das Krankenhaus, 6/ 1994, S. 256- 266.

Riebel, P. (1982): Grundfragen der Kostenrechnung im Gesundheitswesen und ihre Folgerungen für die Ordnungspolitik, in: Gäfgen, Lampert (Hrsg.), 1982, S. 66-72.

Rippel, W., Posinisky, R. (1987): Budgetierung und Controlling in der Anwendung, in: das Krankenhaus, 9/ 1987, S. 347- 352.

Rippel, W., Posinisky, R. (1990): Praktische Anwendung der Kostenarten- und Kostenstellenrechnung, in: das Krankenhaus, 11/ 1990, S. 485- 491.

Rohrig, R., Schnee, S. (1995): Kostenarten, Kostenstellen, Kostenträgerrechnung, in: Eichhorn, S., Schmidt- Rettig, B. (Hrsg.): Krankenhausmanagement im Werteund Strukturwandel, Stuttgart, Berlin, Köln, 1995.

Rossels, H. (1994): Das neue Pflegesatzrecht, lohnt sich der Einsteig schon ab 1995, Contra-Argumentation, in: F\&W, 11 Gangjahr,1994, S. 288-290.

Schäfer, G. (1988): Betriebswirtschaftliche Aspekte in der Krankenhausfinanzierung, herausgegeben von der Fachvereinigung der Verwaltungsleiter deutscher Krankenanstalten., Marl, 1988.

Scheer, A. W. (1996): Prozessmanagement im Krankenhaus, in: Adam, D. (Hrsg.) Krankenhausmanagement, Schriftenreihe zur Unternehmensführung, Band 59, Wiesbaden, 1996, S. 75-96. 
Scherrer, G. (1999): Kostenrechnung, 3., neu bearb. Aufl., Stuttgart, 1999.

Schlüchtermann, J., Gorschlüter, P. (1996): Ausgewählte Aspekte des modernen Kostenmanagements, In Adam, D. (Hrsg.): Krankenhausmanagement, Schriftenreihe zur Unternehmensführung (SzU), Band 59, S. 97 - 112, Wiesbaden 1996.

Schmidt, K. J., Heuser, U. (1993): Das neue Fallpauschalen, Kalkulationsschema, einfach aber ungenau, in: Das Krankenhaus, 10/ 1993, S. 474-476.

Schmidt-Rettig, B. (1995): Vom selbstkostendeckenden Pflegesatz $\mathrm{zu}$ fallpauschalierten Preisen, in: Eichhorn, S., Schmidt-Rettig, B. (Hrsg.): Krankenhausmanagement im Werte- und Strukturwandel, Stuttgart, Berlin, Köln, 1995.

Schmitt, R. (1987): Das Krankenhaus auf dem Weg zum Unternehmen, in: das Krankenhaus, 2/ 1987, S.69-72.

Schmitz, R.M. (1993): Patientenbezogene Steuerung in Krankenhaus, Stuttgart, 1993.

Schoenfeld, H., Möller, H. (1995): Kostenrechnung, Einführung in das betriebswirtschaftliche Rechnungswesen mit Erlöse und Kosten, 8., vollst. überarb. und aktualisierte Aufl., Stuttgart, 1995

Schwartz, Aderea (1997): Informations- und Anreizprobleme im Krankenhaussektor DUV., Wiesbaden, 1997.

Schwarz, K. (1982): Auswirkung der Bevölkerungsentwicklung auf Leistungsnachfrage und Ausgaben im Gesundheitswesen, der Bundesminister für Arbeit und Sozialordnung (Hrsg.), Bonn, 1982.

Schwarz, R. (1993): Modernes Management in öffentlichen Krankenhäusern, wie kann die Krankenhausleitung den Konsequenzen des GSG begegnen, in das Krankenhaus 3/ 1993, S. 116- 120.

Schweitzer, M., Küpper, H. U. (1991): Systeme der Kostenrechnung, 5. Aufl., Landsberg, 1991.

Schweitzer, M., Küpper, H. U. (1995): System der Kosten- und Erlösrechnung, 6. Aufl., München, 1995 
Siebig, J. (1993): Formen der flexiblen Budgetierung im Rahmen der Krankenhausfinanzierung, in: das Krankenhaus, 2/ 1993, S. 91- 95.

Statistisches Bundesamt (1998): Mitteilung für Presse, Erneute Zunahme der Zahl der Patienten in den Krankenhäusern, http://www.statistik-Bund.de/presse/deutsch/pm/p9354094. htm, 01.03.2000, (Stand Am 22.09.1998).

Statistisches Bundesamt (1999 a): (Mitteilung für Presse): Krankenhausstatistik 1998: Mehr Patienten, weniger Betten, kürzere Verweildauer, http://www.statistikBund.de/presse/deutsch/pm/p9354094. htm, 01.03.2000, (Stand am 14.01.1999).

Statistisches Bundesamt (1999 b): Mitteilung für die Presse, Erste Ergebnisse nach dem neuen Europäischen System Volkswirtschaftlicher Gesamtrechnungen (ESVG) 1995 für die Jahre 1991 bis 1998, http://www.statistikbund.de/presse/deutsch/pm/p9152121.htm 01. 03. 2000(Stand am: 28. 04. 1999),

Stauss, B. (1989): Beschwerdepolitik als Instrument des Dienstleistungsmarketing, in: Jahrbuch der Absatz- und Verbrauchsforschung 1989, S. 41 - 62.

Steger, J (1996): Kosten- und Leistungsrechnung, München, 1996.

Steiner, P. (1997): Messung und Beurteilung öffentlicher Leistungen - Der Krankenhausoutput, Sternenfels, Berlin, : Verl. Wiss. Und Praxis, 1997.

Steiner, P. (1998): die Bedeutung der Nachkalkulation für das Kostenqualitäts- und Produktionsmanagement im Krankenhaus, in das Krankenhaus, 11/ 1998, S. 661666.

Strehlau-Schwoll, H. (1993): Anpassung der Kosten- und Leistungsrechnung an die Erfordernisse des GSG - Deckungsbeitragsrechnung und relative Einzelkostenrechnung, in: das Krankenhaus, 5/1993, S. 214-220.

Strehlau-Schwoll, H. (1996): Die Profit-Center Konzeption, Baustein der Führungsorganisation des Krankenhauses, in: F\&W, 4/ 1996, S. 317- 323.

Strehlau-Schwoll, H. (1997): Kostenrechnung und Kostenmanagement im Krankenhaus, in: Freidank, C., Götze, U., Huch, B., Lueter, G., (Hrsg.): Kostenmanagement, Heidelberg, 1997. 
Sukkar, N. (1996): Economic Liberalization in Syria, in: Economic Liberalization and Privatization in Socialist Arab Countries, Hopfinger, H., (Hrsg.), Gotha: Perthes, 1996.

Tanski, J. S. (1997): Finanzierung von Rationalisierungsinvestitionen nach $\S 18 b$ KHG, in: das Krankenhaus, 2/1997, S. 54-58.

Tauch, J. G. (1987): Kosten- und Leistungsrechnung im Krankenhaus von Kostenund Leistungsnachweis zur Patientenbezogenen Kosten- und Leistungsrechnung, Verlag Gütersloher Krankenhaus- Schriften, 1. Aufl., Gütersloh, 1987.

Toso, M. E. (1994): The Value of a Cost Accounting System, in: Finkler, S. A. (Edit.), Issues in Cost Accounting for Health Care Organisations, New York, 1994, S. 5-7.

Trill, R. (1996): Krankenhaus-Management, Aktionsfelder und Erfolgspotentiale, Neuwied, 1996.

Trill, R. (2000): Krankenhaus-Management, Aktionsfelder und Erfolgspotentiale, 2. erweiterte und überarbeitete Aufl. Neuwied, Kriftel: Luchterland, 2000.

Troßmann, E. (1996): Flexible Plankostenrechnung nach Kilger, in: Männel, W. (Hrsg.): Handbuch Kostenrechnung, Wiesbaden, 1996.

Tscheulin, D. K., Hemig, B. (1997): Aufbau einer Kostenträgerrechnung im Krankenhaus, in: das Krankenhaus, 11/ 1997, S. 501- 508.

Tuschen, K. H. (1988): Kosten- und Leistungsrechnung, in: F\&W, JanuarFebruar/ 1988, S. 23-26.

Tuschen, K. H. (1997): 5. Änderungsverordnung zur BPflV, Stand: Regierungsbeschluß, in: Krankenhausumschau, 8/1997, S. 278-282.

Tuschen, K.H., Philippi, M. (1995): Leistung- und Kalkulationsaufstellung im Entgeltsystem der Krankenhäuser, Stuttgart, Berlin, Köln, 1995.

Unterhuber, H. (1986): Preissteuerung in der Krankenhausversorgung. Möglichkeiten und Grenzen der Anwendung von Preisen zur Steuerung der Versorgung mit Krankenhausleistungen, Dissertation an der Bundeswehruniversität München, 1986. 
Von Eiff, W. (1997): Krankenhausbetriebsvergleich, Controllinginstrument zur Planung von Leistungsprozessen, in: das Krankenhaus, 10/ 1997, S. 613- 620.

Wahle, O. (1989): Kostenrechnung II für Studium und Praxis, Ist- und Normalkostenrechnung, 3., überarb. Aufl., Bad Homburg, 1989.

Wedell, H. (1999): Grundlagen des Rechnungswesens, Band 2: Kosten- und Leistungsrechnung, 7., völlig neu bearb. Aufl., Herne, Berlin, 1999.

Wiedermann, R. (1998): Wettbewerb unter Krankenhäusern, Peter Lang, Frankfurt am Main, 1998.

Wöhe, G. (1990): Einführung in die Allgemeine Betriebswirtschaftslehre, 17. Aufl., München, 1990.

World Health Organisation (1980): The Management of Hospitals, Technical Report Series, Geneva: WHO, 1980.

\section{Gesetztexte}

Abgrenzungsverordnung (AbgrV), vom 12. Dezember 1985: Verordnung über die Abgrenzung der im Pflegesatz nicht zu berücksichtigenden Investitionskosten von den pflegesatzfähigen Kosten der Krankenhäuser.

Bundespflegesatzverordnung (BPflV), vom 26. September 1994: Verordnung zur Regelung der Krankenhauspflegesätze.

Gesundheitsstrukturgesetz (GSG) vom 21. Dezember 1992: Gesetz zur Sicherung und Strukturverbesserung der gesetzlichen Krankenversicherung.

Krankenhausbuchführungsverordnung (KHBV), vom 10. April 1978: Verordnung über die Rechnungs- und Buchführungspflichten von Krankenhäusern zuletzt geändert durch die Verordnung zur Neuordnung des Pflegesatzrechts vom 26. September 1994

Krankenhausfinanzierungsgesetz (KHG), vom 26. Mai 1994: Gesetz zur wirtschaftlichen Sicherung der Krankenhäuser und zur Regelung der Krankenhauspflegesätze 


\section{$\underline{\text { Arabische Literatur }}$}

Farruch, O. (1970 Arb.): Die Geschichte der Wissenschaften bei dem Arabern. Dar-Alielm-lelmalaien, Beirut, 1970.

Finanzministerium (1978): Das Syrische Einheitliche Rechnungssystem und Kontenrahmen, das Gesetz Nr. 287 vom 25. 01. 1978, Damaskus, 1978.

Harstanie, H. (1990 Arb.): Krankenhaus-Management, Institut für Management (Hrsg.), Read, Saudiarabien, 1990.

Sakka, H. (1998): das einheitliche Rechnungssystem, 10. Aufl., Universität Damaskus (Hrsg.), Damaskus, 1998.

Universitätsklinik „Assad“‘ Damaskus (1998): Das Handbuch, Damaskus, 1992.

Universitätsklinik „Assad“ Damaskus (1998): Jahresbericht 1997, Damaskus, 1998. 


\section{$\underline{\text { Lebenslauf }}$}

Persönliche Daten Basil Asaad

Geb. am 20.09. 1965 in Syrien

Familienstand: verheiratet, zwei Kinder

Heimat Adresse: Postfach 50059, Damaskus, Syrien

\section{Bildungsgang:}

Schulweg:

1970 - 1982 Grund- und Mittelschule sowie Gymnasium in Syrien,

Stadt Edleb

1982 Abschluss: Hochschulreife (Naturwissenschaftlicher Zweig)

Universität:

1982 - 1986 Universität Damaskus, Wirtschaftswissenschaftsfakultät, 8 Semester. Abschluss : Diplom-Wirtschaftswissenschaften Fachrichtung Rechnungswesen, Präd. „Gut, 67,78 \%“

1986 - 1987 Universität Damaskus, Wirtschaftswissenschaftsfakultät, 2 Semester, Abschluss „Hoch-Studium Diplom II“ in Wirtschaftswissenschaften Fachrichtung Rechnungswesen, Präd. „Gut, 72,16\%“" 1989 - 1993 Universität Damaskus; Wirtschaftswissenschaftsfakultät 7 Semester. Abschluss „Magister“ in Rechnungswesen, Fachgebiet Kostenrechnung für Erdölunternehmen Präd. „Sehr gut, $75 \%$ “

\section{Berufstätigkeiten}

1987 - 1993 Angestellter beim Präsidium der Universität Damaskus (Abrechnungsabteilung).

Seit 12. 1993 wissenschaftlicher Assistent bei der Wirtschaftswissenschaftsfakultät Universität Teschrien, Lattakia, Syrien.

\section{Studium in Deutschland}

10/ 1996 - 3/ 1997 Sprachkurs an der Universität Göttingen Seit 03/ 1997 Doktorand an der Universität Göttingen, Wirtschaftswissenschaftliche Ffakultät, Institut für Betriebswirtschaftliche Produktions- und Investitionsforschung. 


\section{Versicherung}

Ich versichere an Eides Statt, dass ich die eingereichte Dissertation:

Krankenhäuser-spezifische Kosten- und Leistungsrechnung

- Aktuelle Anwendung in Deutschland und Aufbau- Übertragungsmöglichkeiten in anderen Ländern (am Beispiel Syrien) -

selbständig verfasst habe. Anderer als der von mir angegebenen Hilfsmittel und Schriften habe mich nicht bedient. Alle wörtlich oder sinngemäß den Schriften anderer Autoren entnommenen Stellen habe ich kenntlich gemacht.

Göttingen, 13. März 2000 